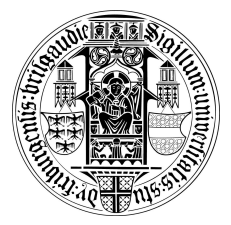

AlBerT-Ludwigs-UniversitäT

FrEIBURG I. BR.

FAKUltät FÜR MAThematiK UND Physik

Mathematisches Institut • Abteilung für Angewandte Mathematik

\title{
Development of an Efficient 3-D CFD Software to Simulate and Visualize the Scavenging of a Two-Stroke Engine
}

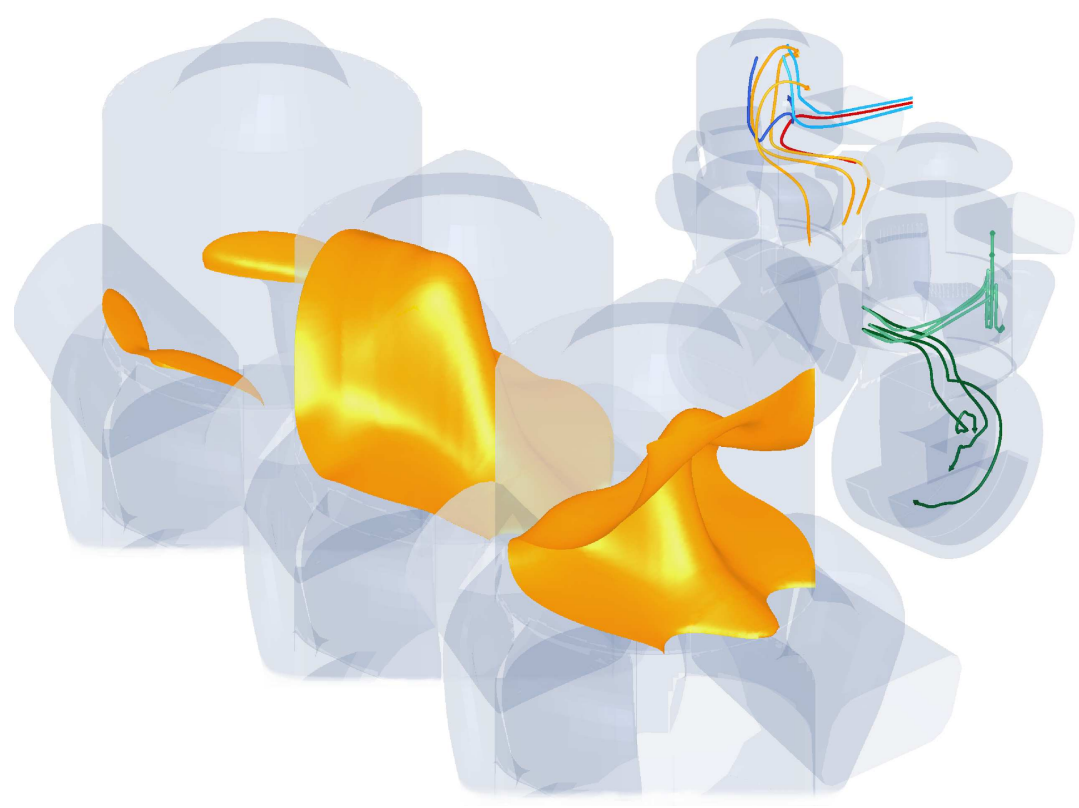

DiRK TREscher

Dissertation zur Erlangung des Doktorgrades der Fakultät für Mathematik und Physik der Albert-Ludwigs-Universität Freiburg im Breisgau

Betreuer: Prof. Dr. Dietmar Kröner

Freiburg im Breisgau

Juni 2005 
Dekan:

Referenten:
Prof. Dr. J. Honerkamp

Prof. Dr. D. Kröner

Prof. Dr. M. Lukacova

Datum der Promotion: 29. August 2005

Picture on title page:

The series of three pictures on the left hand side of the image depicts an iso-surface of freshgas concentration in the two-stroke engine cylinder after opening of the transfer ports, at the lowest position of the piston, and shortly before closing of the transfer ports (from top to bottom). On the right hand side, different particle traces through the cylinder (top) and the crankcase (bottom) are shown. 


\section{Preface}

The two-stroke engine is widely used in many different devices. But the environmentally problematic exhaust characteristic is a big drawback of this type of engine. In the scavenging process, when the fresh charge displaces the exhaust gas in the cylinder, lies the biggest opportunity to improve the exhaust data by geometry optimization. In order to give the engineer the opportunity to analyze the flow field and to compare different engine geometries, we developed a software package to efficiently simulate the three-dimensional, time-dependent, Navier-Stokes equations within the complex, real-world geometry of the two-stroke engine with moving piston and rotating crankshaft.

\section{Acknowledgements}

First I want to thank especially Prof. Dr. Dietmar Kröner for giving me this very interesting thesis and supporting me during all this time. I also want to thank Dr. Mario Ohlberger, Dr. Andreas Dedner, Dr. Matthias Wesenberg, Robert Klöfkorn, Dennis Diehl and Christian Merkle for fruitful discussions and constructive input. Dr. Axel Klimmek was always a great help when discussing application details. My former office colleague Rolf-Thomas Happe also contributed to the completion of this work. And Eldad Louw had the last word concerning the English language.

This thesis was partly supported by the BMBF, the 'Bundesministerium für Forschung und Technologie', under the reference number $03 \mathrm{KRM} 1 \mathrm{FR}$, and the 'Graduiertenkolleg Nichtlineare Differentialgleichungen: Modellierung, Theorie, Numerik, Visualisierung' of the German Research Association DFG, the 'Deutsche Forschungsgemeinschaft'. 



\section{Contents}

$\begin{array}{ll}\text { Preface } & \text { iii }\end{array}$

Acknowledgements

1 Introduction 1

1.1 History and Applications . . . . . . . . . . . . . . . . 1

1.2 Advantages and Problems . . . . . . . . . . . . . . . . . 1

1.3 Why CFD? . . . . . . . . . . . . . . . . . 3

1.3.1 Why Computational Simulations? . . . . . . . . . 3

1.3.2 Why 3-D Fluid Dynamics? . . . . . . . . . . . . 3

1.4 State of the Art . . . . . . . . . . . . . . 3

1.5 The Setting of the Two-Stroke Engine . . . . . . . . . . . . . 4

1.6 This Thesis . . . . . . . . . . . . . . . . 4

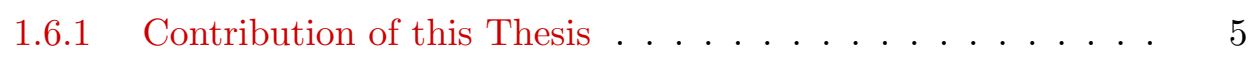

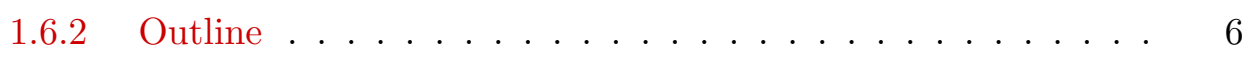

1.6.3 Third Party Software .............. 7

2 The Two-Stroke Engine 9

2.1 Notations . . . . . . . . . . . . . . . . . . . 9

2.2 Method of Operation . . . . . . . . . . . . . . . 9

2.2.1 The Different Parts of the Two-Stroke Engine . . . . . . . . 9

2.2.2 Description of the Cycle of Operation . . . . . . . . . . . . 10

2.3 Important Characteristics . . . . . . . . . . . . . . . . . . . 11

2.3.1 Engine Geometry . . . . . . . . . . . . . . . . 12

2.3.2 Thermodynamic Terms . . . . . . . . . . . . . 13

2.4 The Scavenging Process . . . . . . . . . . . . . . . . . 15

2.4.1 Different Scavenging Methods . . . . . . . . . . . . . . 15

2.4.2 Early Theories of Scavenging . . . . . . . . . . . . . . 18

2.5 The Otto Cycle . . . . . . . . . . . . . . . . . . . 20

2.5.1 Theoretical Case . . . . . . . . . . . . . . . . 20

2.5.2 Comparison with Experimental Data . . . . . . . . . 20

2.5.3 Indicated Mean Effective Pressure . . . . . . . . . . . . . 21

2.6 Combustion . . . . . . . . . . . . . . . . . . . 21

2.6.1 The Combustion Process in Spark Ignition (SI) Engines . . . 21

2.6 .2 Heat Release . . . . . . . . . . . . . . . . . . 22

2.6 .3 Exhaust Gas . . . . . . . . . . . . . . . . . 22 
2.7 Evaluation of Numerical Results . . . . . . . . . . . . . . . 23

2.7.1 Characteristics . . . . . . . . . . . . . 23

2.7.2 Jante Test . . . . . . . . . . . . . . . . . . . 24

2.7.3 Sharp vertical Velocity Boundary . . . . . . . . . . 25

2.7.4 Deviation of the Flow Direction . . . . . . . . . . . 25

2.7 .5 Squish Velocity . . . . . . . . . . . . . . 26

3 Physical Background $\quad 27$

3.1 Definition of the Determining Variables of a Fluid . . . . . . . . . 27

3.2 The Material Properties . . . . . . . . . . . . . . . . . . . 28

3.2.1 The Heat Capacities $c_{p}$ and $c_{\mathrm{V}} \ldots \ldots \ldots \ldots 28$

3.2.2 The Adiabatic Exponent $\gamma$. . . . . . . . . . . . . . 29

3.2 .3 The Speed of Sound $c \ldots \ldots \ldots$

3.2 .4 The Viscosity $\eta \ldots \ldots \ldots \ldots$

3.2 .5 The Thermal Conductivity $\lambda \ldots \ldots \ldots$

3.2.6 The Reynolds and Prandtl Numbers . . . . . . . . . . . . . . 30

3.3 The Navier-Stokes Equations . . . . . . . . . . . . . . . . . . . . . 31

3.3 .1 The Euler Equations . . . . . . . . . . . . . . . . . 31

3.3.2 The Navier-Stokes Equations . . . . . . . . . . . . . . 32

3.4 Initial Conditions and Boundary Conditions . . . . . . . . . 32

3.4 .1 Initial Conditions . . . . . . . . . . . . . . . 32

3.4 .2 Boundary Conditions . . . . . . . . . . . . . . 33

3.5 The Equations of State . . . . . . . . . . . . . . 36

3.5.1 The Thermal Equation of State . . . . . . . . . 36

3.5.2 The Caloric Equation of State . . . . . . . . . . . . . 36

3.6 Ideal Gas - Real Gas . . . . . . . . . . . . . . . . . . . 37

3.7 Gas Mixtures . . . . . . . . . . . . . . . . . . . 37

3.7 .1 Notations . . . . . . . . . . . . . . . . 37

3.7.2 The Equations of State . . . . . . . . . . . . . 38

3.7.3 Fresh Gas, Exhaust Gas . . . . . . . . . . . . . . . . 38

3.7.4 The Influence of the Air-Fuel Ratio . . . . . . . . . . . . 39

3.8 Temperature Dependency of the Material Properties . . . . . . . . . 40

3.8 .1 Notations . . . . . . . . . . . . . . . . . . 40

3.8 .2 Formulas ......................... 41

4 The Numerical Scheme 45

4.1 Notations . . . . . . . . . . . . . . . . . . 45

4.2 The Mesh Structure . . . . . . . . . . . . . . . . 46

4.3 The Finite Volume Scheme . . . . . . . . . . . . . . . . 47

4.3 .1 The Time Derivative . . . . . . . . . . . . . . . . 48

4.3 .2 The Numerical Fluxes . . . . . . . . . . . . . . . . . . . . 49

4.4 The Convective Flux . . . . . . . . . . . . . . . . . 50

4.5 The Viscous Flux . . . . . . . . . . . . . . . . . . . . . . 52

4.64 .6 The Time-Dependent Integral Form . . . . . . . . . . . . . 54

4.7 The Boundary Conditions . . . . . . . . . . . . . . . . . 55

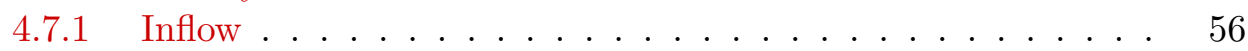

4.7 .2 Outflow ........................ 57 
4.7 .3 Slip (fixed) . . . . . . . . . . . . . . . . 57

4.7 .4 No-Slip (fixed) . . . . . . . . . . . . . . . 57

4.7 .5 No-Slip (moving) . . . . . . . . . . . . . . 57

4.8 The CFL-Condition . . . . . . . . . . . . . . . . 58

4.8 .1 The Convective Part . . . . . . . . . . . . 58

4.8 .2 The Viscous Part . . . . . . . . . . . . . . . . . 59

4.8.3 The Overall Time-Step . . . . . . . . . . . . . . . 60

4.9 The General Equation of State . . . . . . . . . . . . . . 60

4.9.1 The Relaxation System . . . . . . . . . . . . . 60

4.9 .2 The Numerical Scheme . . . . . . . . . . . . . . . . . 62

4.9.3 Tabularized Equation of State . . . . . . . . . . . . 63

4.10 The Algorithm of the Main Numerical Scheme . . . . . . . . . . 63

5 Special Mesh Treatment $\quad \mathbf{6 5}$

5.1 Grid Merging . . . . . . . . . . . . . . . . . 65

5.1 .1 Different Techniques . . . . . . . . . . . . . 65

5.1.2 Matching Interfaces with the Patched Grid Scheme . . . . 66

5.1 .3 The Structure . . . . . . . . . . . . . . . 67

5.1 .4 The Algorithm . . . . . . . . . . . . . . . . . 67

5.1.5 The Massflow Problem . . . . . . . . . . . . . 68

5.2 The Piston Motion . . . . . . . . . . . . . . . . . 72

5.2 .1 Different Techniques . . . . . . . . . . . . . . 72

5.2.2 Detailed Description of the Snapper Algorithm . . . . . . . 73

5.2 .3 The Extended Snapper Algorithm . . . . . . . . . . . 74

5.2.4 The Update of the Window Area to the Ducts . . . . . . . . 76

5.2 .5 The Algorithm . . . . . . . . . . . . . 76

5.3 The Crankshaft Motion . . . . . . . . . . . . . . 78

5.3 .1 Different Techniques . . . . . . . . . . . . . 78

5.3.2 Exploitation of the Quasi 2D Structure . . . . . . . . . 82

5.3.3 Compensation of the Artificial Transport . . . . . . . . . . 83

5.3.4 The Crankshaft-Transfer Port Interface . . . . . . . . . . 84

5.3 .5 The Algorithm . . . . . . . . . . . . . 85

5.4 The Connecting Rod . . . . . . . . . . . . . . . . 85

$\begin{array}{llr}6 & \text { Reducing Computational Time } & \mathbf{8 7}\end{array}$

6.1 Temporally Consistent Adaptive Local Time-Stepping . . . . . . . . 87

6.1.1 The Time-Step Level . . . . . . . . . . . . . . . . 87

6.1.2 Forced Update of an Element . . . . . . . . . . . . . 89

6.1.3 Time Level Restrictions . . . . . . . . . . . . . . . . 89

6.1.4 Elements in the Rotating Crankshaft Mesh . . . . . . . . . . 89

6.1.5 The Algorithm . . . . . . . . . . . . . . . . . . . 89

6.2 Dynamic Local Mesh Adaption . . . . . . . . . . . . . . . . . . 91

6.2.1 Different approaches . . . . . . . . . . . . . . . 91

6.2.2 The Mesh Structure . . . . . . . . . . . . . . . . . 91

6.2.3 Mesh Adaption Criteria . . . . . . . . . . . . . . . . 92

6.2 .4 The Marking Strategy . . . . . . . . . . . . . . . . . . . . 94

6.2.5 Special Mesh Considerations . . . . . . . . . . . . 95 
6.2.6 The Algorithm . . . . . . . . . . . . . . . . . . 97

6.3 Accelerated Convergence by Enhanced Initial Data . . . . . . . . . . 97

6.3.1 Convergence in Time for Periodic Problems . . . . . . . . . . 97

6.3.2 Criteria for the Convergence in Time . . . . . . . . . . . . 99

6.3 .3 The Mesh Iteration . . . . . . . . . . . . . . . . . . . 99

6.3.4 Mesh Dependency Checking . . . . . . . . . . . . . . . 100

7 Parallelization $\quad 101$

7.1 Efficiency of a Parallel Algorithm . . . . . . . . . . . . . . . . . . . 101

7.2 Parallelization Concepts . . . . . . . . . . . . . . . . . 102

7.2.1 Distributed Memory Architectures . . . . . . . . . . . . . . . 102

7.2.2 Shared Memory Architectures . . . . . . . . . . . . . . . 103

7.2 .3 Hybrid Architectures . . . . . . . . . . . . . . . . . . . 103

7.3 The OpenMP Parallelization . . . . . . . . . . . . . . 103

7.3.1 The Finite Volume Scheme . . . . . . . . . . . . . . . . 103

7.3 .2 The Mesh Routines . . . . . . . . . . . . . . . . . . . . 104

7.4 The Extended Partitioning Algorithm . . . . . . . . . . . . . 104

7.5 Dynamic Load Balancing . . . . . . . . . . . . . . . . 105

8 The Connecting Rod - 2D Estimations $\quad 107$

8.1 Description of the Problem . . . . . . . . . . . . . . 107

8.2 The Model . . . . . . . . . . . . . . . . . . . . . 107

8.2.1 The Compression of the Crankcase . . . . . . . . . . . 108

8.2.2 The Inlet and Outlet Boundary Conditions . . . . . . . . . . 108

8.2.3 The Motion of the Connecting Rod . . . . . . . . . . . . . . 109

8.3 Meshes . . . . . . . . . . . . . . . . . . . 109

8.4 Results . . . . . . . . . . . . . . . . . . 110

9 Validation of the Software 113

9.1 Evaluation of a Numerical Scheme . . . . . . . . . . . . . . . 113

9.1.1 The Experimental Order of Convergence . . . . . . . . . . . 113

9.1.2 The Efficiency of a Scheme . . . . . . . . . . . . . . . 114

9.2 Inviscid Flow . . . . . . . . . . . . . . . . . . . . . 114

9.2.1 Compression Profile . . . . . . . . . . . . . . . . . . . . 114

9.2 .2 Sod's Shock Tube . . . . . . . . . . . . . . . . . 117

9.2 .3 Nozzle Flow . . . . . . . . . . . . . . . . . . . . . 122

9.2.4 Channel with a Bump . . . . . . . . . . . . . . . . 125

9.3 Viscous Flow . . . . . . . . . . . . . . . . . . . . . . . . 129

9.3.1 Poiseuille Flow . . . . . . . . . . . . . . . . . . . . . . . . 129

9.3 .2 Couette Flow . . . . . . . . . . . . . . . . . . . . 133

9.3.3 Blasius Plate . . . . . . . . . . . . . . . . 135

9.3.4 Traveling Wave . . . . . . . . . . . . . . . . . . . . . . . . 141

9.4 Mesh Issues . . . . . . . . . . . . . . . . . . . . . . . . . . . 145

9.4.1 Grid Merging: A Sudden Expansion . . . . . . . . . . . . . . 145

9.4.2 Dynamic Mesh Adaption: The Forward Facing Step . . . . . 148

9.4.3 Boundary Fitting of the Dynamic Mesh Adaption . . . . . . 152

9.5 In the Two-Stroke Engine Context . . . . . . . . . . . . . . . . 158 
9.5.1 Straight Pipe attached to a Cylinder . . . . . . . . . . 158

9.5.2 Temperature-dependent Material Properties: The Otto Cycle 163

10 Application: The Two-Stroke Engine 167

10.1 Meshes . . . . . . . . . . . . . . . . . . 167

10.1.1 Generation . . . . . . . . . . . . . 167

10.1.2 Different Size Meshes . . . . . . . . . . . . . . . . 168

10.1.3 An Alternative to a Transparent Boundary Condition . . . . 169

10.2 Configuration . . . . . . . . . . . . . . . . . 170

10.2 .1 Geometrical Data . . . . . . . . . . . . . . . 170

10.2.2 Temperature-dependent Material Properties . . . . . . . . 170

10.2 .3 Combustion Data . . . . . . . . . . . . . . . . . . 172

10.3 Initial and Boundary Data . . . . . . . . . . . . . . 172

10.3.1 Initial Conditions . . . . . . . . . . . . . . . 172

10.3.2 Boundary Conditions . . . . . . . . . . . . . . . 173

10.4 Parallelization . . . . . . . . . . . . . . . . . 173

10.4.1 Partitioning . . . . . . . . . . . . . . 173

10.4.2 Performance Analysis . . . . . . . . . . . . . . . 173

10.5 Visualization and Characteristical Diagrams . . . . . . . . . . 175

10.6 Results . . . . . . . . . . . . . . . . . 176

10.6.1 Convergence in Time . . . . . . . . . . . . . 176

10.6.2 Convergence for Mesh Width $h \rightarrow 0 \ldots \ldots \ldots$

10.6.3 Comparison with Measured Data . . . . . . . . . . . . . 179

10.6.4 Visualization of the Flow Structure . . . . . . . . . . 179

10.6.5 Characteristical Diagrams . . . . . . . . . . . . 186

10.6 .6 Jante Test . . . . . . . . . . . . . . . . . . . 190

10.6.7 Sharp Vertical Velocity Boundary . . . . . . . . . . . . . 190

10.6.8 Deviation Angles . . . . . . . . . . . . . . . . . . . . . 190

10.6 .9 Squish velocity . . . . . . . . . . . . . . . 191

10.7 Efficiency of the Adaptive Strategies . . . . . . . . . . . . . . 192

10.7.1 Efficiency of the Adaptive Local Time-Stepping . . . . . . . 192

10.7.2 Efficiency of the Dynamic Local Mesh Adaption . . . . . . . 194

10.8 The Execution Time Call-Graph . . . . . . . . . . . . . . . . 195

10.9 Question One: The Effect of the Crankshaft Rotation . . . . . . . . 195

10.9.1 Setup of the Comparison . . . . . . . . . . . 195

10.9 .2 Results . . . . . . . . . . . . . . . . . 197

10.10 Question Two: The Unsymmetrical Transfer Port . . . . . . . . . . 197

10.10.1 Setup of the Comparison . . . . . . . . . . . . . 199

10.10 .2 Results . . . . . . . . . . . . . . . . . . . . 199

10.11 Question Three: The Study of Short-Circuiting . . . . . . . . . . . . 199

10.11.1 Enhanced Fresh-Gas Tracking _. . . . . . . . . . . 200

10.11 .2 Results . . . . . . . . . . . . . . . 200

11 Summary and Outlook 203

11.1 Conclusion . . . . . . . . . . . . . . . . . . 203

11.2 Further Steps . . . . . . . . . . . . . . . . . . . 204 
$\begin{array}{lr}\text { Appendices } & 205\end{array}$

$\begin{array}{ll}\text { A Tables } & 205\end{array}$

B The Material Properties $\quad 209$

$\begin{array}{ll}\text { C The Software Package engine_flow } & 211\end{array}$

$\begin{array}{ll}\text { List of Figures/Tables } & 215\end{array}$

$\begin{array}{lr}\text { Bibliography } & 220\end{array}$

$\begin{array}{lr}\text { Index } & 229\end{array}$ 


\section{Chapter 1}

\section{Introduction}

\section{$1.1 \quad$ History and Applications}

The two-stroke engine was invented by Sir Dugald Clerk in England at the end of the $19^{\text {th }}$ century. In 1891, the forerunner of the model which is in use up to now, was patented by Joseph Day in England.

At first the two-stroke engine was mainly used in motorcycles. In the late $19^{\text {th }}$ and beginning of the $20^{\text {th }}$ century some successful race bikes were powered by such units. In the 1930s the first supercharged machines appeared and since 1959 the two-stroke engine with a tuned exhaust pipe and a disc valve induction system can be found in many motorcycles. Today these engines are used in high numbers in scooters for general transportation and recreation although they are in some cases replaced by the four-stroke engine due to legislative pressure for reduced emissions.

Further very important applications are hand-held power tools such as chainsaws, blowers or brush-cutters. The two-stroke engine with its excellent power to weight ratio (see Section 1.2) is ideally suited for this kind of tool.

Other applications include marine outboard motors, recreational products like snowmobiles and water scooters, microlight aircrafts and small electricity generating sets.

Apart from a few remaining two-stroke cars from former Eastern Germany ("Trabi") the usage of this engine for automobiles has disappeared due to high exhaust emissions and fuel consumption rates but research and development is being conducted even in this field because of the low acid emissions of two-stroke engines.

A completely different engine with respect to size but with a similar construction principle is the two-stroke compression ignition (CI) or diesel unit. The range of applications includes truck and aircraft engines with high specific power output to the usually some $12 \mathrm{~m}$ tall marine diesel main propulsion unit, which produces about 4000 hp per cylinder.

\subsection{Advantages and Problems}

Usually people have the bad odor of two-stroke powered devices in mind when thinking about this kind of engine. 

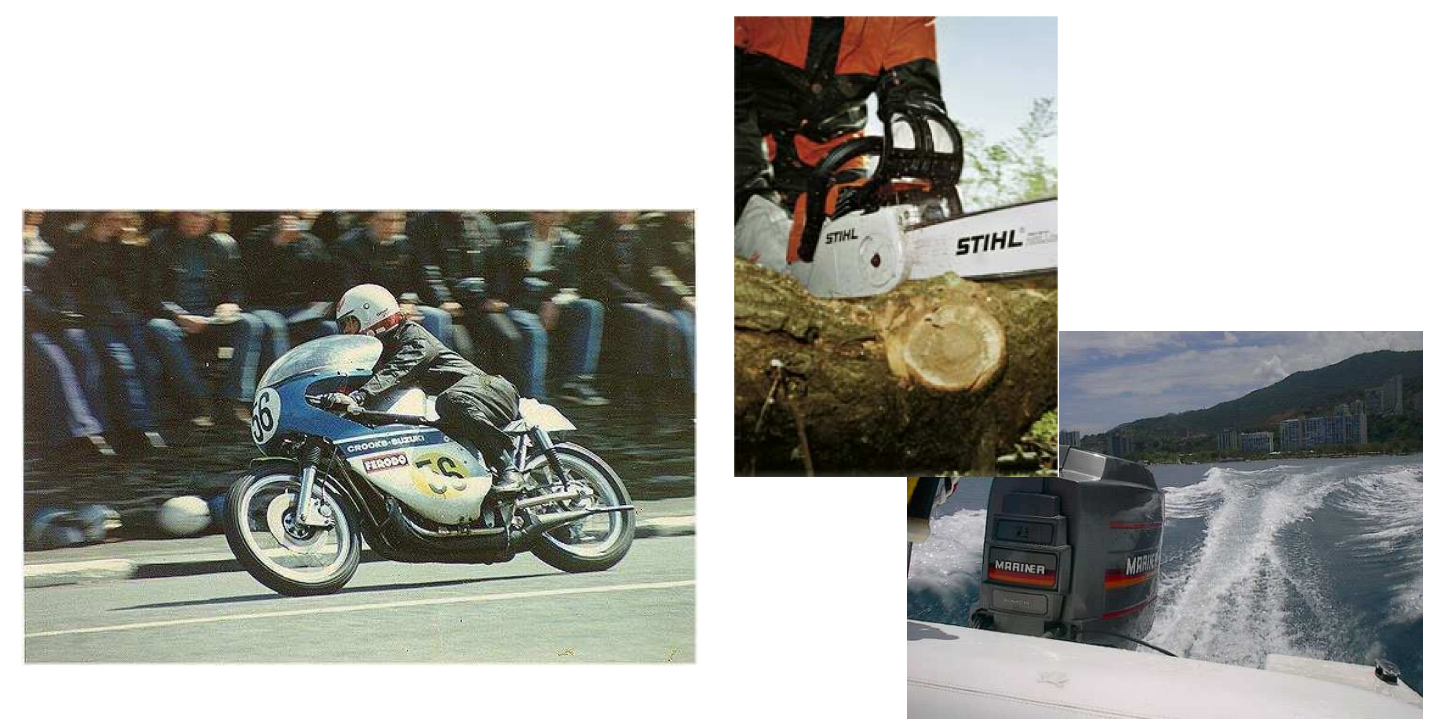

Figure 1.1.1: Typical applications of two-stroke engines.

The bad exhaust gas characteristics of two-stroke engines stem from different sources. The short-circuiting in the scavenging process results in an emission of unburnt hydrocarbons. At rich mixture combustion the insufficient air leads to an exhaust emission of carbon monoxide and also unburnt hydrocarbons. And finally, the total-loss lubrication, mixed into the fuel, is the cause of smoke emissions at cold start-up and at light load. ${ }^{1}$

Two-stroke engines also usually do not last as long as four-stroke engines. The lack of a dedicated lubrication system means that the parts of a two-stroke engine wear faster.

However, there are some important characteristics of two-stroke engines that just cannot be matched by their opponents, the four-stroke engines:

- Two-stroke engines usually do not need valves, which simplifies their construction, reduces their complexity, and finally their size and weight.

- Two-stroke engines fire once every revolution (four-stroke engines fire once every other revolution) - this gives two-stroke engines a significantly higher power to weight ratio.

- Because of the missing lubrication system, two-stroke engines can work in any orientation, which is very useful in a device like a chainsaw.

- Because of the "inbuilt" lubrication, no extra maintenance of the oil lubrication is necessary.

\footnotetext{
${ }^{1}$ However, this has been improved a lot since better materials, lubricants and lubrication methods have become available, and the fuel-oil ratios applied come close to the ones found in four-stroke engines.
} 
- The scavenge process in a two-stroke engine always leaves a remainder of exhaust gas in the cylinder. This acts as a damper on the formation of nitrogen oxides and the engine therefore has very low acid exhaust emissions. Four-stroke engines need an exhaust gas recirculation (EGR) which again adds mechanical complexity.

\subsection{Why CFD?}

\subsubsection{Why Computational Simulations?}

The measurement of the characteristics of a prototype is an effective research and development tool. But for the early stages of the development of a new engine it is very costly and laborious to build many prototypes. In order to test different possibilities within a short period of time and with an acceptable financial effort it is necessary to employ computational simulations.

\subsubsection{Why 3-D Fluid Dynamics?}

The most efficient possibility for reducing the pollutant emission is to minimize the loss of scavenging. By means of the numerical simulation of gas motion in the cylinder, one can determine which changes of the geometry of the cylinder, transfer ducts, and exhaust port can improve these scavenging characteristics. Onedimensional models cannot capture the complexity of the flow pattern within the cylinder and are therefore not suited for geometrical optimization. However, the analysis conducted with these one-dimensional models can provide a good starting point for an in-depth study.

Although three-dimensional computations usually take a lot longer than a onedimensional calculation, a further reason in favor of the three-dimensional simulation is the possibility of visualizing each detail of the flow at an arbitrary time. A detailed analysis of flow behavior is possible.

These topics will be discussed in detail in the following chapters.

\subsection{State of the Art}

What has been done to quite an extent (see e.g. [LSG+94, Mit02, RK97, RKPM01, Wer00, ZSLC04]), is the analysis of two-stroke engines with commercial software. The big drawback of this method is the "black-box"-character of these software packages. The source code is obviously not available and detailed description of the used algorithms is sparse. Furthermore, this kind of software is designed for a multitude of applications and special properties of the two-stroke engine cannot be taken into account. A further disadvantage is the error tolerance of these products. Occurring errors are generally ignored and not taken as an indication that something went wrong. The debugging possibilities are therefore very limited and one is left with a calculated flow pattern without any idea of convergence.

Although it has to be admitted that these commercial codes have made quite an impressive progress during the last few years, the user still has to be careful how to interpret the obtained results. Usually, some comparison of characteristics of 
the predicted flow and measurements is undertaken, but whether the accuracy of the simulation, e.g. the number of degrees of freedom, itself is sufficient is rarely checked. Until now no software is able to obtain an accurate solution for all flows. So it is always vital to analyze the obtained results and study the effects of approximations one had to introduce in order to be able to solve the underlying system of equations. If, for example, one tries to simulate the Navier-Stokes equations and is not using an appropriate mesh with sufficient boundary refinement, one will not obtain the desired flow structure. And if one does not know about the approximations made it is difficult to estimate the accuracy of the result.

"Industrial users of commercial CFD codes should especially be careful, as the optimism of salesmen is legendary."

\section{J.H. Ferziger, M. Perić [FP97]}

On the other hand there are lots of very refined methods being developed to analyze fluid flow behavior in very idealistic settings. Many research codes have emerged, and have been thoroughly tested, to solve flow problems very efficiently on simple geometries for model problems. These techniques have also been analyzed on a theoretical mathematical basis. Methods like local adaptive time-stepping, local adaptive grid refinement and parallelization have been used to speed up calculations. Higher order schemes have been constructed to achieve a higher accuracy of the simulation.

The software engine_flow which was developed within the scope of this thesis is an approach to apply these new and refined methods of fluid simulation to the complex, real-world problem of the two-stroke engine.

\subsection{The Setting of the Two-Stroke Engine}

Now we want to briefly outline the proportions in which the simulation of the two-stroke engine should take place. Our intention is to compute the flow of fresh gas through the crankcase, its way into the cylinder, and the displacement of the exhaust gas out of the cylinder. The fuel in the fresh charge is assumed to be already vaporized. Thus, the material properties of air and exhaust gas are used. The two-stroke engine that is simulated, has a size of $50 \mathrm{ccm}$ and speed of 9000 rpm, typical for hand-held devices. Therefore, a Reynolds and Prandtl number (see Paragraph 3.2.6 on page 30) of around

$$
\operatorname{Re} \approx 50000 \text { and } \operatorname{Pr} \approx 0.71
$$

are the characteristic values for our fluid. (For further details of the values that were used in the simulation see Section 10.2 on page 170.)

\subsection{This Thesis}

In this thesis we want to describe in detail how the task of numerically solving the flow through a two-stroke engine with moving parts is solved in an efficient way. 
The mathematical model behind the scenes is illuminated and the used numerical schemes are specified.

Also, our aim is to show what is possible with our software package and what is not, what is the accuracy that can be expected of the algorithm. Because only with this information in mind can one judge the obtained results in an adequate way.

For more in-depth information concerning the program structures, the function and precise implementation of the single subroutines compare the documentation of the engine_flow-software available from www.mathematik.uni-freiburg.de/ IAM/homepages/trescher/.

\subsubsection{Contribution of this Thesis}

With this software package we want to enable engineers to reduce the environmental pollution of two-stroke engines by providing the means for calculating and visualizing the structure of the time-dependent flow field within the engine, and by simulating the effects of geometry changes on the characteristics of the engine. Therefore, the simulation needs to be as accurate as possible within a reasonable computational time. This includes the usage of assembled complex meshes that mirror the CAD-data of the engine geometry. The simulation of the moving parts in the two-stroke engine also needs the application of appropriate algorithms.

The basic algorithm has already been used with great success in an industrial application. Since then it has been extended by modern numerical methods in the framework of very complex, real-world, moving meshes.

First, the computation of the convective flux function is carried out by the AUSMDV Riemann solver, which has been proven to be very efficient in comparison to other schemes. Then the introduction of the temperature dependency of the material properties of the fluid, namely heat capacity, adiabatic exponent, viscosity and thermal conductivity, has augmented the realistic setting within the compression and expansion of the hot gas within the cylinder. This temperature dependency of the heat capacity causes a change in the equation of state. The gas is not polytropic any more but calorically imperfect. Thus, the use of a relaxation method is necessary in order to retain our Riemann solver. To increase the efficiency of the treatment of the caloric equation of state, a tabularized computation of this equation of state is implemented.

To account for the complex geometry, it was necessary to realize a special mesh treatment. The computational domain can be assembled by different meshes that are connected in a mass conservative way. These meshes do not need to match at the connecting interfaces. Furthermore, the piston and crankshaft motion is obtained by very efficient algorithms.

In order to speed up the computation of the numerical solution, different strategies have been followed. Adaptive local time-stepping has been implemented in a time consistent manner, thus taking into consideration the very inhomogeneous size of the mesh elements. Additionally, a dynamic local mesh adaption with hanging knots (therefore having a non-conformal mesh) and an efficient error estimator are used to reach a better resolution in critical areas. The necessary complex interaction of the mesh adaption algorithm with the treatment of the moving meshes has been handled. A further reduction in computational time has been obtained 
by the parallelization of the numerical scheme and the mesh routines. To handle this parallelization of the mesh treatment, an extended partitioning for the dynamic load balancing has been implemented. All in all, the calculations that used to take several weeks can now be conducted in less than a day with even greater accuracy.

The software has been thoroughly tested on several test cases for the single modules. Finally, a simulation of flow through a real-world geometry of an existing two-stroke engine has been performed, the results have been validated with measured pressure data for this engine, and the flow has been qualitatively and quantitatively studied.

Therefore, with this software it is possible to analyze a given two-stroke engine geometry in a fast and extensive way. The structure of the three-dimensional flow pattern at the scavenging of the cylinder can be observed with the help of several different visualization techniques, also in a time-dependent manner. By these means, it is possible to detect problematic areas of the engine geometry and to study possible improvements. Further on, the calculated diagrams of characteristic values allow an estimation of the quality of the engine and a quantitative comparison of two or more different geometries.

\subsubsection{Outline}

In the following chapter an introduction to the two-stroke engine is given. All parts of the engine and their function in the cycle of operation are described. The central geometrical and thermodynamic terms are introduced and the most important process for optimization, the scavenging process, is explained. The Otto Cycle and the process of combustion are introduced. The numerically relevant section about the evaluation of results rounds off this chapter.

The physical background of the description of the fluid flow is the content of Chapter 3. The fundamental variables of a fluid are introduced followed by the system of the Navier-Stokes equations characterizing the properties of a time-dependent viscous compressible flow. The necessary initial and boundary conditions complete the system together with the equations of state. This is followed by a discussion of the special topics of real gas, gas mixtures, and temperature-dependency of the material properties.

The finite volume scheme, which is the basis of the numerical treatment of the Navier-Stokes equations, is presented in Chapter 4. Before this is motivated, the underlying mesh structure is illustrated. Then the discretization of the convective and viscous fluxes, as a central part in the numerical scheme, is considered and the treatment of the different boundary conditions is described. Finally, the CFL condition, which determines the time-step size, and the handling of the equations of state of a calorically imperfect gas with a relaxation scheme are shown.

The complex geometry with its moving parts requires a special mesh treatment as given in Chapter 5. The main topics include the grid merging techniques, the realizations of the piston motion and crankshaft rotation. The description of the connecting rod completes this Chapter.

As every CFD simulation is very demanding concerning the computational resources, methods for a reduction of computational time are discussed in Chapter 6. Three main techniques are presented, namely temporally consistent adaptive 
local time-stepping, the dynamic local mesh adaption and an iterative process for accelerating the convergence in time.

Chapter 7 deals with an even further speed-up of the simulation by parallelization. The notion of efficiency with respect to parallel algorithms and different concepts of parallelizations is introduced before we give the details of our parallel implementation, conforming to the OpenMP standard. Finally, the extended partitioning algorithm and the dynamic load balancing are described.

In the following three chapters, beginning with Chapter 8, the developed software is applied to different problems. First, the influence of the connecting rod to the flow pattern in the cylinder during the scavenging process is evaluated. For this, a 2-D model of the crankcase is devised, which captures the movement of the connecting rod and all other important processes, like compression and time-dependent boundary conditions. The discussion of the results finishes this chapter.

Chapter 9 consists of the description of several test cases in order to validate the derived software. First, the notion of performance of a numerical scheme is clarified. Then the validation is conducted for inviscid flow, viscous flow, mesh related issues, and in the context of the two-stroke engine.

The simulation of the full real-world two-stroke engine is carried out in Chapter 10. A detailed description of the mesh generation, the configuration, the initial and boundary data, and further parallelization issues are given. The possibilities of analyzing the flow with several visualization techniques and by means of characteristical diagrams are presented. The obtained results of the simulations are discussed in depth, and the efficiency of the algorithms is demonstrated. Finally, three important application questions are studied: the effect of the crankshaft rotation, the influence of an unsymmetric transfer port, and an enhanced fresh-gas tracking.

The summary of the achieved results and the outlook towards things to be done in the future, in Chapter 11, complete this thesis.

\subsubsection{Third Party Software}

Some parts of the software were developed with the help of the integrated programming environment KDevelop (www.kdevelop.org/) of the KDE environment under SUSE Linux. For a runtime profiling the very useful program KCachegrind in combination with Callgrind (kcachegrind.sourceforge.net/cgi-bin/show.cgi) was employed.

The documentation of the software was greatly simplified by the Doxygen documentation system (www.stack.nl/ dimitri/doxygen/).

For the visualization software viewer the GRAPE visualization library ([Gra99]) has been used extensively. The $2-\mathrm{D}$ graphs have been plotted with the plotting tool Grace (plasma-gate.weizmann.ac.il/Grace/).

Most of the meshes have been generated using the commercial software ICEM CFD ([Ice04]).

Finally this thesis has been processed with the typesetting program $\mathrm{AT}_{\mathrm{E} X} \mathrm{X}$, its extensions, and its front-end LyX. 


\section{Chapter 2}

\section{The Two-Stroke Engine}

\section{$2.1 \quad$ Notations}

Table 2.1: Notations in the context of the two-stroke engine

\begin{tabular}{|cl|}
\hline symbol & name \\
\hline \hline tdc & crankshaft position at top dead center \\
bdc & crankshaft position at bottom dead center \\
btdc & before top dead center \\
atdc & after top dead center \\
bbdc & before bottom dead center \\
abdc & after bottom dead center \\
rpm & speed of rotation (rotations per minute) \\
$d_{\mathrm{b}}$ & cylinder bore (Definition 2.3.2) \\
$l_{\mathrm{S}}$ & cylinder stroke (Definition 2.3.3) \\
$D R$ & delivery ratio (Definition 2.3.11) \\
$S R$ & scavenging ratio (Definition 2.3.12) \\
$S E$ & scavenging efficiency (Definition 2.3.13) \\
$\Pi$ & Purity (Definition 2.3.14) \\
$T E$ & trapping efficiency (Definition 2.3.15) \\
$A F R$ & air-to-fuel ratio (Definition 2.3.16)
\end{tabular}

\subsection{Method of Operation}

\subsubsection{The Different Parts of the Two-Stroke Engine}

The two-stroke engine consists of several ports and chambers (see Figure 2.2.1 on the next page). The ports (inlet, transfer, and exhaust ports) are used to channel 
the flow from one chamber to the next. They are opened and closed by the motion of the piston, which acts as valves. In the chamber below the piston (the crankcase), the fresh gas is compressed to enable the transfer into the cylinder above the piston, where the combustion and power process takes place.

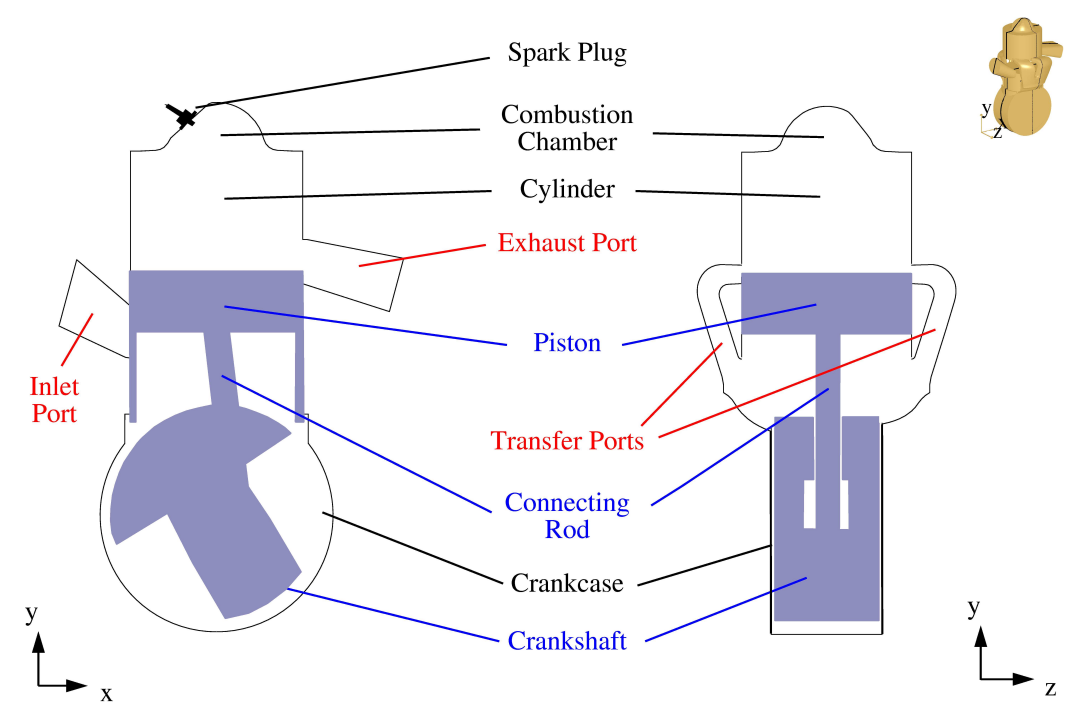

Figure 2.2.1: Components of a two-stroke engine.

\subsubsection{Description of the Cycle of Operation}

To clarify the processes taking place in a two-stroke engine, we will follow the gas flow all the way through the engine.

The fresh gas coming from the carburator enters the crankcase via the opened inlet port due to the upward motion of the piston, which lowers the pressure inside the crankcase below the atmospheric value (Figure 2.2.2 (a) on the facing page).

Then the fresh charge is compressed in the crankcase as the inlet port is closed by the lower edge of the piston (Figure 2.2.2 (b)).

As soon as the piston has moved far enough downwards the transfer ports are opened and the fresh charge enters the cylinder since the pressure in the crankcase exceeds the cylinder pressure (Figure 2.2.2 (c)). In the cylinder the fresh gas displaces the burnt exhaust gas, referred to as the scavenge process. This is a critical point in the two-stroke process. If the transfer ports are badly directed then a part of the fresh gas can exit from the cylinder directly into the exhaust port (called "shortcircuiting"). Therefore, this fresh gas is lost for the next combustion and contributes heavily on the emission rate of unburnt hydrocarbons.

On its upward motion, the piston again closes the transfer and exhaust ports, and the trapped gas is compressed in the cylinder (compression stroke). Shortly before the piston reaches its uppermost position (Figure 2.2.2 (d)) the combustion begins with the ignition of the spark plug, producing a rapid rise in temperature and pressure driving the piston down on the power stroke. 
When the exhaust port is opened, the still over-pressured hot exhaust gas is released into the exhaust port as a pressure shock-type wave and from there into the silencer (Figure 2.2.2 (b)).

On opening of the transfer ports the exhaust gas is replaced by the fresh charge (Figure 2.2.2 (c)). The reflection of the pressure wave of the initial pulse of exhaust gas at the tapered shape of the exhaust pipe can be used to push the fresh gas, which has already arrived at the exhaust port, back into the cylinder (not shown in Figure 2.2.2). However, this is difficult to achieve when there is not much space available for an extended exhaust pipe.

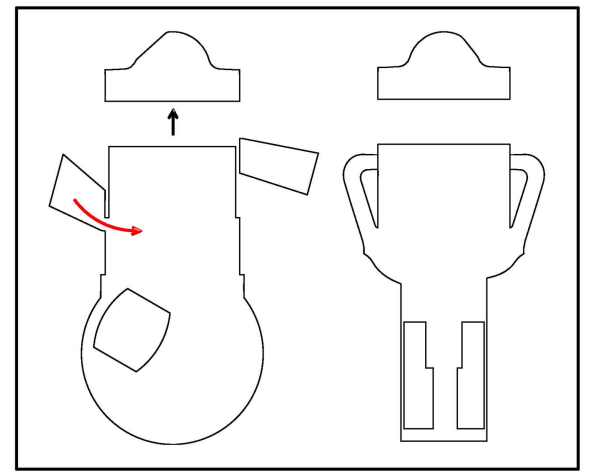

(a) Compression

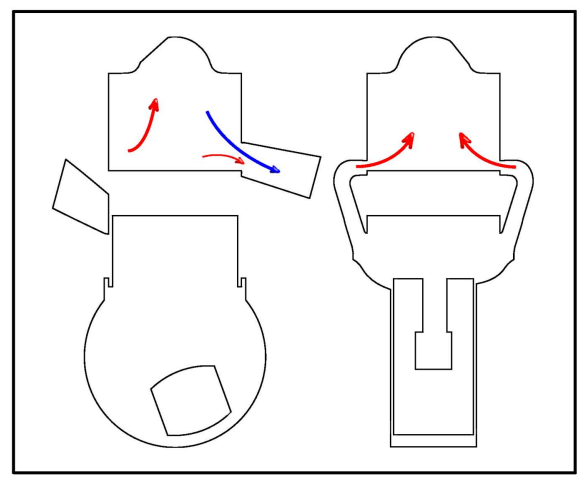

(c) Scavenging Process

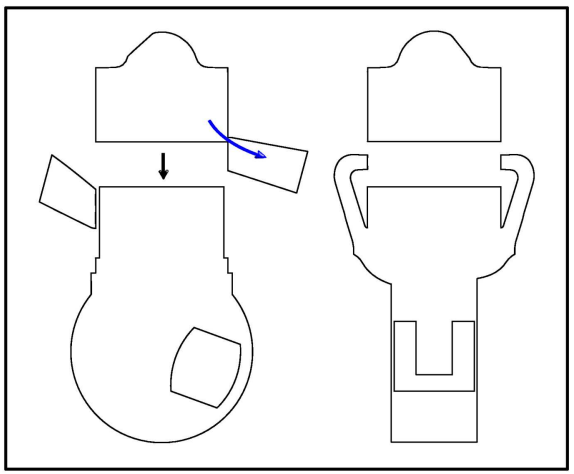

(b) Blowdown / Fresh Charge Compression

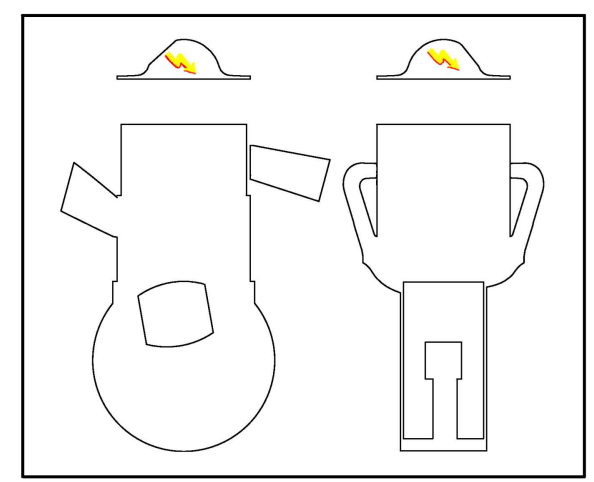

(d) Ignition / Combustion

Figure 2.2.2: Different phases in the cycle of a two-stroke engine.

\subsection{Important Characteristics}

For a thoroughly quantitative measurement of engine behavior it is necessary to define the important characteristics of two-stroke engine development. 


\subsubsection{Engine Geometry}

First, we will summarize some important geometrical properties of the two-stroke engine (see also Figure 2.3.1 on the facing page).

Definition 2.3.1. $(t d c, b d c)$ The top dead center $(t d c)$ is reached when the piston is at its uppermost position, similarly at bottom dead center $(b d c)$ the piston is at its lowest position.

Definition 2.3.2. $\left(d_{\mathrm{b}}\right)$ The bore $d_{\mathrm{b}}$ is defined as the diameter of the cylinder.

Definition 2.3.3. $\left(l_{\mathrm{s}}\right)$ The stroke $l_{s}$ is the distance between the position of the piston at bdc and at tdc.

Definition 2.3.4. $\left(l_{\mathrm{ts}}\right)$ The trapped stroke $l_{\mathrm{ts}}$ is the distance between the position of the piston at exhaust port closure and at tdc.

Definition 2.3.5. $\left(V_{\mathrm{s}}\right)$ The swept volume $V_{s}$ of one cylinder is then given by

$$
V_{\mathrm{s}}:=\pi\left(\frac{d_{\mathrm{b}}}{2}\right)^{2} l_{\mathrm{s}} .
$$

Definition 2.3.6. $\left(V_{\mathrm{ts}}\right)$ The trapped swept volume $V_{\mathrm{ts}}$ of one cylinder is calculated analogously from

$$
V_{\mathrm{ts}}:=\pi\left(\frac{d_{\mathrm{b}}}{2}\right)^{2} l_{\mathrm{ts}} .
$$

All compression ratio values are the proportions of the maximum volume in a chamber to its minimum volume. Therefore, we have the following:

Definition 2.3.7. $\left(C R_{\mathrm{cc}}\right)$ The crankcase compression ratio $C R_{\mathrm{cc}}$ is

$$
C R_{\mathrm{cc}}:=\frac{V_{\mathrm{cc}}+V_{\mathrm{s}}}{V_{\mathrm{cc}}}
$$

with the crankcase volume $V_{\text {cc }}$ at bdc.

Definition 2.3.8. $\left(C R_{\mathrm{g}}\right)$ If $V_{\mathrm{c}}$ is the clearance volume above the piston at tdc, the geometric compression ratio $C R_{g}$ of the cylinder is given by

$$
C R_{\mathrm{g}}:=\frac{V_{\mathrm{c}}+V_{\mathrm{s}}}{V_{\mathrm{c}}} .
$$

The actual compression process does not occur until the exhaust port is closed, therefore one defines:

Definition 2.3.9. $\left(C R_{\mathrm{t}}\right)$ The trapped compression ratio $C R_{\mathrm{t}}$ of the cylinder is

$$
C R_{\mathrm{t}}:=\frac{V_{\mathrm{c}}+V_{\mathrm{ts}}}{V_{\mathrm{c}}} .
$$


In order to calculate the piston position $h_{p}$ from the crankshaft angle $\Theta$, we need to introduce the connecting rod length $l_{\mathrm{cr}}$ and the crank throw $l_{\mathrm{ct}}$, which are illustrated in Figure 2.3.2 on the next page. As is easily seen the crank throw is obtained by $l_{\mathrm{ct}}=\frac{l_{\mathrm{s}}}{2}$.

Lemma 2.3.10. (Piston position and velocity) Then the piston position $h_{p}(\Theta)$ can be calculated from

$$
h_{p}(\Theta)=l_{c r}+l_{c t}(1-\cos \Theta)-\sqrt{l_{c r}^{2}-\left(l_{c t} \sin \Theta\right)^{2}} .
$$

The (oriented) piston velocity is given by

$$
v_{p}(\Theta)=-\frac{r p m}{60} 2 \pi l_{c t} \sin (\Theta)\left(1+\frac{l_{c t}}{l_{c r}} \cos (\Theta) \frac{1}{\sqrt{1-\left(\frac{l_{c t}}{l_{c r}} \sin (\Theta)\right)^{2}}}\right) .
$$

Proof. This is verified by standard trigonometric calculus.

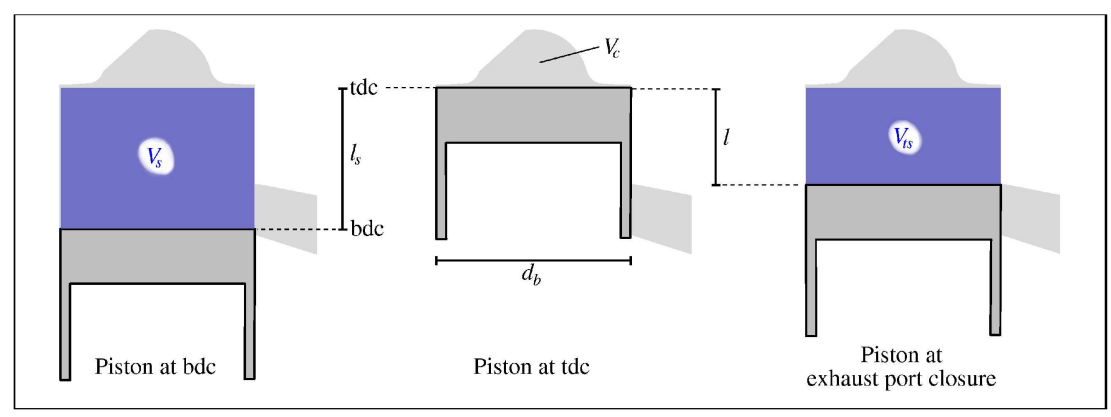

Figure 2.3.1: Geometrical properties of the two-stroke engine.

\subsubsection{Thermodynamic Terms}

To be able to measure the scavenge process quantitatively (see Paragraph 2.7.1 on page 23), one needs to introduce further characteristic values.

Definition 2.3.11. $(D R)$ The delivery ratio $D R$ is defined as

$$
D R:=\frac{m_{\mathrm{f}}}{m_{\mathrm{sv}}},
$$

where $m_{\mathrm{f}}$ is the mass of fresh air supplied during the scavenge process and $m_{\mathrm{sv}}:=$ $\rho_{\mathrm{at}} V_{\mathrm{s}}$ is the mass of air (under atmospheric conditions) to fill the swept volume.

Definition 2.3.12. $(S R)$ In contrast to the delivery ratio, the scavenge ratio $S R$ is a function of $m_{\mathrm{ev}}:=\rho_{\mathrm{at}}\left(V_{\mathrm{s}}+V_{\mathrm{c}}\right)$, the mass of air to fill the entire cylinder volume (under atmospheric conditions),

$$
S R:=\frac{m_{\mathrm{f}}}{m_{\mathrm{ev}}} .
$$




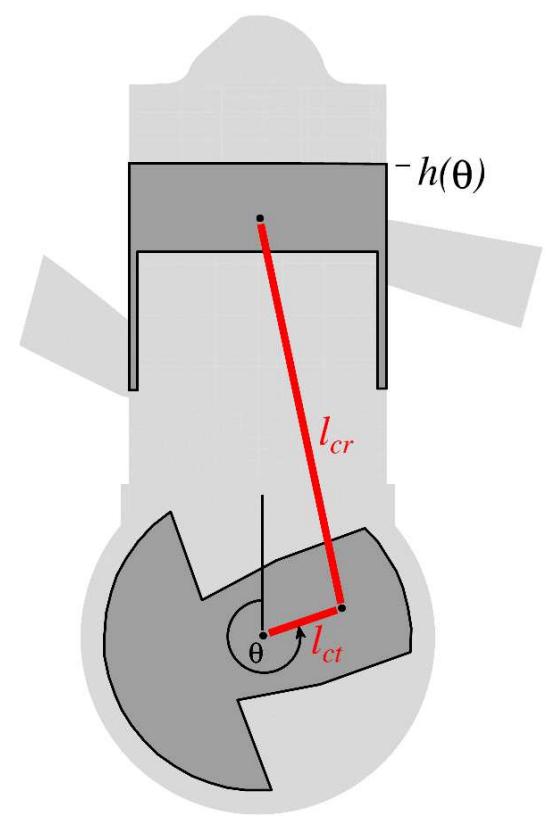

Figure 2.3.2: Position of the piston.

Definition 2.3.13. ( $S E$ ) The scavenging efficiency $S E$ is defined as the mass of fresh air $m_{\text {trf }}$ that has been trapped by comparison with the total mass of charge $m_{\text {tr }}$ in the cylinder at exhaust port closure:

$$
S E:=\frac{m_{\mathrm{trf}}}{m_{\mathrm{tr}}}
$$

The total trapped mass $m_{\mathrm{tr}}$ is composed of the fresh charge trapped $m_{\mathrm{trf}}$, the exhaust gas trapped $m_{\text {trex }}$, and the unburnt air $m_{\text {trr }}$ from the previous cycle: $m_{\mathrm{tr}}:=m_{\mathrm{trf}}+$ $m_{\text {trex }}+m_{\text {trr. }}{ }^{1}$

Definition 2.3.14. ( $\Pi$ ) The Purity $\Pi$ is the ratio of the mass of the trapped fresh charge $m_{\mathrm{trf}}+m_{\mathrm{trr}}$ to the total mass of the trapped gas $m_{\mathrm{tr}}:^{2}$

$$
\Pi:=\frac{m_{\mathrm{trf}}+m_{\mathrm{trr}}}{m_{\mathrm{tr}}}
$$

Definition 2.3.15. (TE) The trapping efficiency $T E$ is the ratio of the mass of fresh air that has been captured $m_{\text {trf }}$ to that of the air supplied $m_{\mathrm{f}}$ :

$$
T E:=\frac{m_{\mathrm{trf}}}{m_{\mathrm{f}}}
$$

The next definition deals with the composition of the fresh gas that comes from the carburator via the inlet duct to the crankcase.

\footnotetext{
${ }^{1} m_{\text {trr }}$ is assumed to be zero in our simulation, we suppose a complete combustion which is plausible in a spark ignition engine with a not too lean mixture (see also Section 2.6.3 on page 22).

${ }^{2}$ Because of our assumption $m_{\text {trr }}=0$, we have $S E=\Pi$.
} 
Definition 2.3.16. $(A F R)$ The air-fuel ratio $A F R$ is the proportion of mass of air to that of the fuel delivered.

The AFR for the so-called "stoichiometric" combustion, i.e. a complete combustion of all fuel available with the exact amount of oxygen present in the fresh charge, is between 14.2 and 14.9 depending on the type of fuel. A rich mixture is a mixture with an $A F R$ smaller than stoichiometric $A F R$ with a rich misfire limit of about 9 and a lean mixture has an $A F R$ bigger than stoichiometric $A F R$ with a lean misfire limit of about 18 .

For further information on this subject refer to [B196].

\subsection{The Scavenging Process}

Scavenging in a two-stroke engine is the process of gas exchange in the cylinder. The fresh gas is introduced by the transfer ports and this displaces the exhaust gas out of the exhaust port. The most important problem, which causes the bad exhaust characteristics of two-stroke engines, is the short-circuiting of a part of the fresh gas right out of the exhaust, without being burnt in the combustion process.

Therefore, the development of good scavenging for the two-stroke engine is essential in order to reduce fuel consumption levels, to meet the ever-increasing demands of international legislation for exhaust emissions, and most importantly to decrease the pollution of our environment as much as possible.

\subsubsection{Different Scavenging Methods}

\section{Loop Scavenging}

As opposed to the original method of scavenging, where the piston crown is used as a deflector for the fresh charge (cross scavenging in Section 2.4.1 on the following page), in loop scavenging the main transfer port is directed away from the exhaust port to minimize short-circuiting (see Section 2.2.2 on page 10). This way, a flat piston crown can be used to avoid the overheating of sharp edges on its upper side. A compact combustion chamber, made possible by a flat piston, also permits an efficient combustion process.

Some port layouts of loop scavenging engines are shown in Figure 2.4.1 on the following page. Beside the main ports there may also be rear ports and side ports, and there exists a big variety in different port layouts. But the main port controls the scavenge process, therefore, it is necessary to consider its orientation carefully. In Figure 2.4.3 on page 17, the angles of the horizontal port direction, $\alpha_{\mathrm{h} 1}$ and $\alpha_{\mathrm{h} 2}$, the points of encounter, $P_{1}$ and $P_{2}$, and the up-sweep angle $\alpha_{\mathrm{v}}$ are displayed. What has been shown in experiments ([B196]) and can be seen in the numerical simulation of the engine geometry in Chapter 10 on page 167 as well, is the deviation of the real flow from these geometric angles (see also Paragraph 2.7.4 on page 25).

Another consideration has to be made about the usage of "handle"-type or "open" transfer ports. The open ports are on their inner side open towards the piston, whereas the handle-type ports resemble a tea cup handle and are not connected to the piston all the way (see Figure 2.4.2 on the following page). 
Because of the many possibilities in port layout and the complexity of the flow situation in loop scavenging, empirical advice for the choice of the different geometric values is greatly enhanced by three-dimensional CFD calculations.

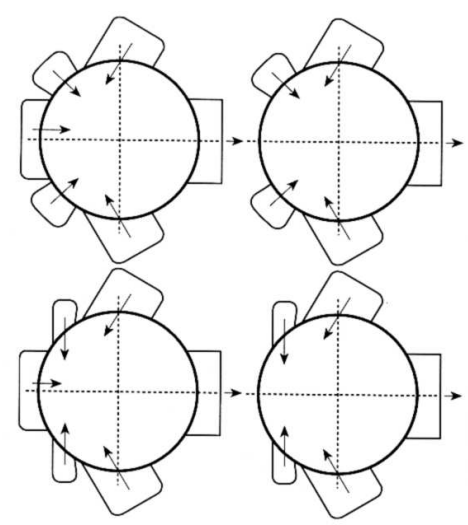

Figure 2.4.1: Different port plan layouts of loop scavenged engines (image taken from [B196]).
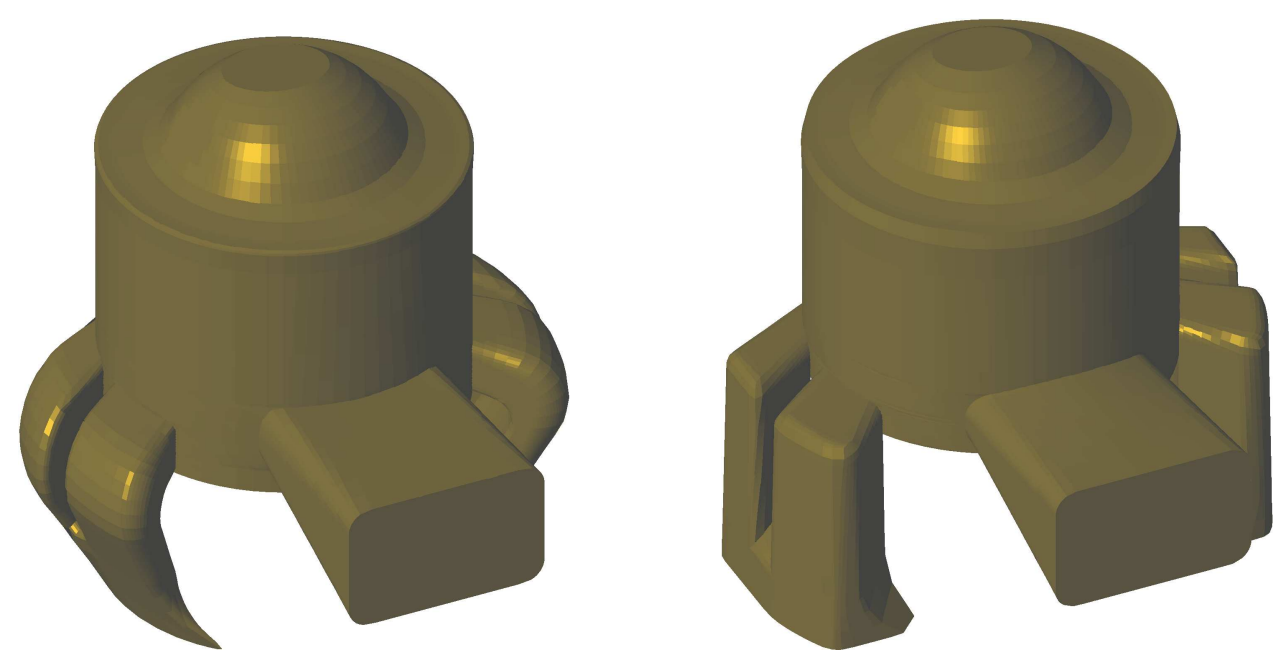

Figure 2.4.2: Handle-type and open-type transfer ports.

\section{Cross Scavenging}

In cross scavenging the transfer ports are opposite the exhaust port and the flow of fresh charge is directed upwards by a deflector piston (as shown in the left part of Figure 2.4.4 on the next page). Because of this port layout, a better packing in multi cylinder engines is possible. Furthermore, the manufacturing is simplified by this layout as the ports can be drilled in one work step. On the other hand, the hotrunning deflector edges of the piston crown present a potential for pre-ignition and 


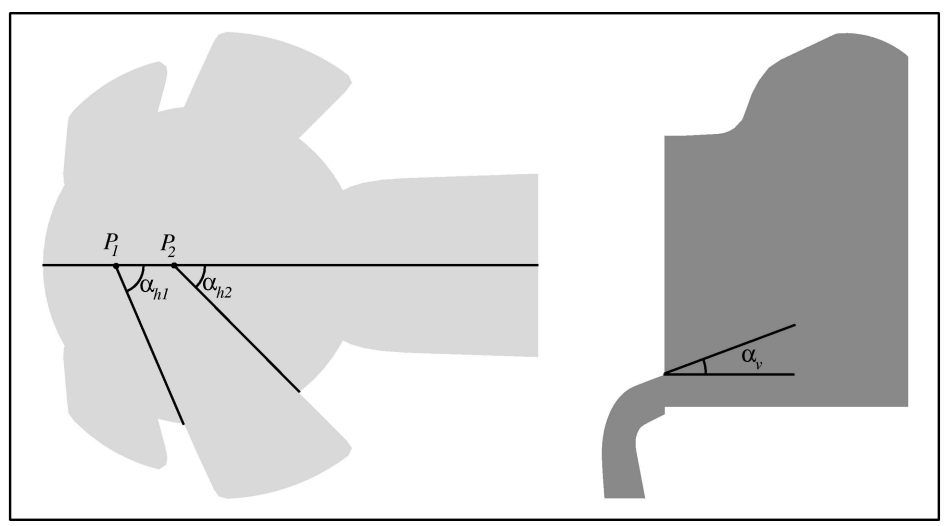

Figure 2.4.3: Horizontal (left) and vertical (right) port orientations.

detonation (cf. Section 2.6 on page 21), and the non-compact combustion chamber is not ideal for an efficient combustion.

As the flow is mainly directed by the deflector piston crown and not by the interaction of jets of air, the need for an accurate computational simulation of the scavenge process is not so essential in cross scavenging. Simple design rules, which are set out in [B196], are usually enough to optimize the scavenging in this type of engine.

\section{Uniflow Scavenging}

The principle of uniflow scavenging is to induce the fresh charge at one end of the cylinder while removing the exhaust gas from the other, as can be seen in the right part of Figure 2.4.4. Usually valves are needed to operate either the inlet or the outlet ports. Since uniflow scavenging is most efficient in big diesel engines with bores exceeding $1000 \mathrm{~mm}$ the increased mechanical complexity in general rules out the usage of this scavenging method for smaller lightweight engines.
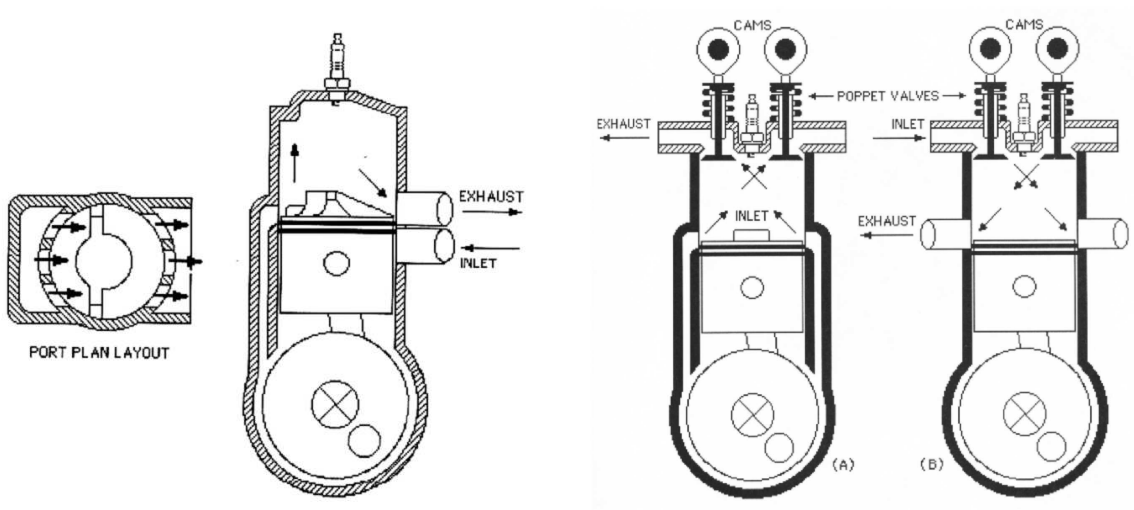

Figure 2.4.4: Cross scavenging (left) and uniflow scavenging (right) (images taken from [B196]). 


\section{Scavenging with the Help of Valves}

To use the motion of the piston to control all port timing events is the simplest method of controlling the flow through the engine. In this case the opening and closing of the ports is symmetric to tdc (and bdc). More sophisticated possibilities include the usage of different types of valves. This permits an asymmetrical operation of the inlet and outlet timing and gives the designer more control over the intake and exhaust system. However, as in the case of uniflow scavenging, this control must be paid by the higher mechanical complexity.

\subsubsection{Early Theories of Scavenging}

The resulting (volume related) scavenge ratio-trapping efficiency characteristics of the models described in the following are given in Figure 2.4.5 on the facing page.

\section{Assumptions}

The simple theories of scavenging all need some idealizations. Before the computer age these theories were used to calculate scavenge properties "by hand". Since the emerging equations had to be easily solvable the assumptions of constant cylinder volume, constant cylinder temperature (isothermal), and constant pressure (isobaric) during the scavenging process were made.

\section{Perfect Displacement Scavenging}

This theory requires all of the exhaust gas in the cylinder to be driven out by the inflowing fresh charge. No mixing of the two gases takes place and no shortcircuiting is possible until all of the exhaust gas has been replaced by the fresh gas. This situation is depicted in the left part of Figure 2.4.6 on the next page.

\section{Perfect Mixing Scavenging}

Contrary to the aforementioned concept, in this scavenging model the fresh charge entering the cylinder mixes "perfectly" with the exhaust gas. Thus, the gas in the cylinder is a uniform mixture of fresh gas and exhaust gas at every instant. This means, as soon as fresh charge flows into the cylinder it partly exits into the exhaust port as seen in the right part of Figure 2.4.6.

\section{Combined Models}

A refined strategy was to incorporate both kinds of models. Perfect displacement was assumed to take place until a certain threshold of fresh charge $S R_{d m}$ in the cylinder was reached. At this moment the gas was mixed "instantaneously" and the perfect mixing model was applied from there onwards.

\section{Inclusion of Short-Circuiting}

A further extension was to include the short-circuiting into the scavenging calculations. A part $\sigma$ of the incoming scavenging flow exits directly through the exhaust 
duct without displacing or mixing with the exhaust gas.

\section{Advantage of CFD Calculations}

In the aforementioned models the different scavenging methods, as uniflow, loop or cross scavenging, are accounted for only in the choice of the parameters $S R_{d m}$ and $\sigma$. Also, these parameters obviously have to change when e.g. port direction angles are altered. But up to now there is no empirical evidence on how these parameters are related to geometric characteristics. Thus, it is not possible to use these models to optimize the port layout in a two-stroke engine to achieve a better scavenging process. In these models it is not possible to capture the most important part of scavenging.

This is the big advantage of a three-dimensional simulation of the flow situation in the cylinder during scavenging. All the geometrical data is incorporated in the simulation, detailed scavenge ratio-trapping efficiency characteristics can be plotted and, furthermore, it is possible to watch the flow at every instant of time. The problems of the actual scavenging can be visualized, e.g. short-circuiting can be detected and even traced back to the transfer port which caused it (see Section 10.11 on page 199).

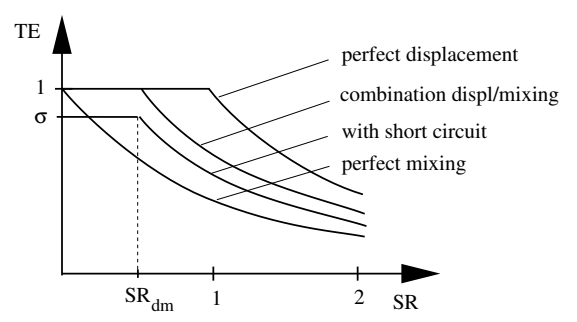

Figure 2.4.5: SR-TE diagram of the different simple scavenging models.
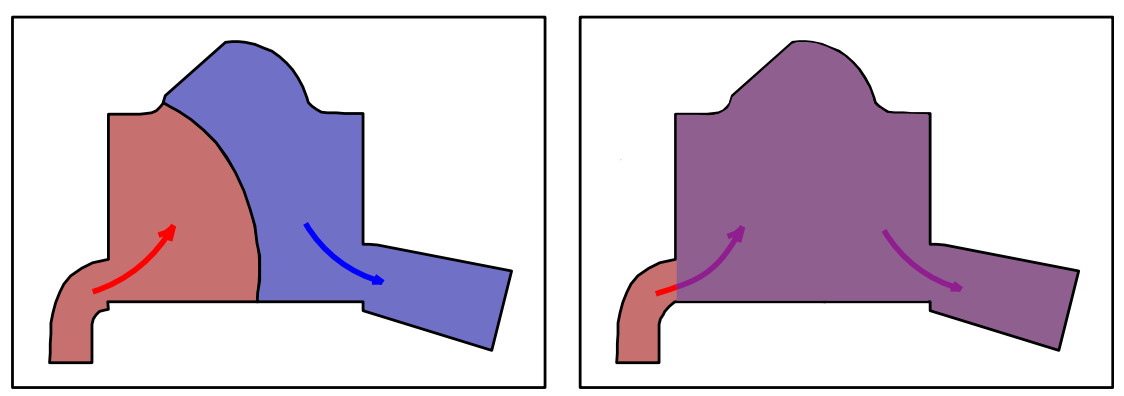

Figure 2.4.6: Perfect displacement (left) and perfect mixing (right) in the cylinder. 


\subsection{The Otto Cycle}

This is the thermodynamic cycle of the two-stroke engine. The fresh gas is compressed and combusted, after which the burnt gas expands and is released from the cylinder.

This cycle, which takes place mostly after the scavenging has finished, has to be modeled in the CFD simulation in order to derive the correct preconditions for the next scavenging process.

\subsubsection{Theoretical Case}

Conjecture 2.5.1. Under the following assumptions the Otto Cycle can be calculated theoretically:

- The compression does not begin before trapping.

- The compression and expansion process occur under ideal isentropic conditions with air as working gas, which means that pressure $p$ and volume $V$ are related by $p V^{\gamma}=$ const with the constant adiabatic exponent $\gamma=1.4$ of air (see Section 3.2.2 on page 29).

- All heat release (combustion) takes place at tdc at constant volume.

- All heat rejection occurs at exhaust port opening.

The result of the easy calculation of the thermodynamic cycle is sketched in Figure 2.5.1.
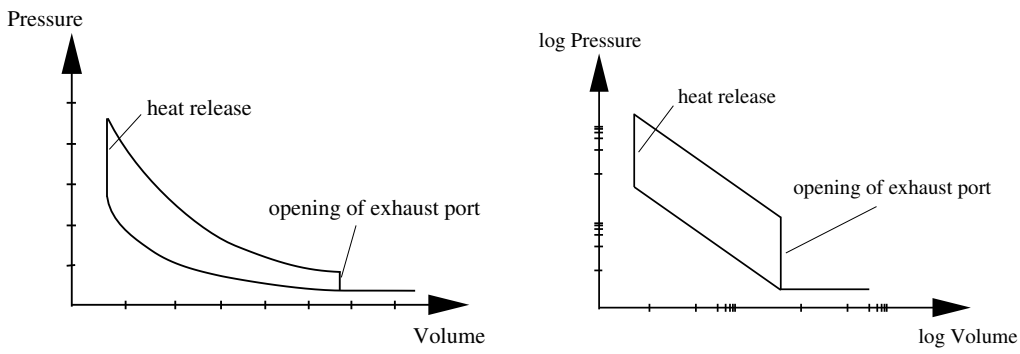

Figure 2.5.1: Draft of the theoretical Otto Cycle.

\subsubsection{Comparison with Experimental Data}

The measured pressure-volume data of an actual engine cylinder reveals that the assumptions of the theoretical model are not valid.

- The compression process begins shortly after bdc, clearly before the exhaust port closure. 
- The compression and expansion are not isentropic nor polytropic $\left(p V^{c} \neq\right.$ const for $c=$ const ).

- The process of combustion takes $40^{\circ}-60^{\circ}$ crankshaft angle of time (see the next Section 2.6).

- Also, the heat release is not an instantaneous process.

For a comparison of the numerical experiment refer to test case 9.5.2 on page 163 and to Chapter 10 on page 167.

\subsubsection{Indicated Mean Effective Pressure}

The work of the gas in the thermodynamic cycle is transmitted to the crankshaft by the piston and the connecting rod. The ideal ${ }^{3}$ work produced is

$$
\text { Work produced }=\int F d x=\int p A d x=\int p d V
$$

with force $F$ created from the gas pressure $p$, distance $x$, piston area $A$ and swept volume $V$. Therefore, the work produced per cycle is the cyclic integral for one crankshaft revolution from tdc to tdc. This is the enclosed area on the pressurevolume diagram in Figure 2.5.1 on the facing page.

Definition 2.5.2. (imep) The indicated mean effective pressure (imep) is defined as the average pressure relating to the swept volume $V_{s}$ :

$$
\text { imep }=\frac{1}{V_{s}} \int p d V
$$

with a cyclic integral from tdc to tdc.

The imep is useful in relating the engine performance status of two engines independent of their swept volume.

\subsection{Combustion}

\subsubsection{The Combustion Process in Spark Ignition (SI) Engines}

As was already pointed out in the previous section, combustion is a phenomenon that does not take place instantaneously at tdc. Instead, it is a process at a flame front started by a spark plug burning its way through a fuel-air mixture. ${ }^{4}$ The flame front velocity lies between 20 and $50 \frac{\mathrm{m}}{\mathrm{s}}$, which is sufficiently rapid to burn the entire fuel at even high engine speeds.

\footnotetext{
${ }^{3}$ Without friction or leakage past the piston.

${ }^{4}$ This is clearly to be distinguished from a detonation which is many orders of magnitude faster and results in a very sharp local rise of temperature and pressure.
} 


\subsubsection{Heat Release}

The heat released by the combustion of the fuel can be seen thermodynamically as a heat addition to a closed system. There are two methods to calculate the total heat release:

- The amount of fuel in the cylinder at ignition time can be calculated, multiplied by the lower calorific value $C_{f l}$ of the fuel used to get the amount of heat available in the fuel. Then the total heat release is given by the product of the combustion efficiency $\eta_{c}$ and the heat available in the fuel. The drawback of this method is the uncertainty of the value of the combustion efficiency $\eta_{c}$, which depends on many factors and varies with different loads of the engine.

- The total heat release can also be calculated from the pressure difference of measured (with combustion) and calculated (without combustion) pressure after the combustion process. The disadvantage of this approach is that the heat release is available only after the combustion has finished. But because of the identical pressure of the real and the simulated engine this is also a good technique to calibrate the conditions within the cylinder just before the scavenging starts.

We are interested mainly in optimizing the scavenge process of the engine. The combustion has to be integrated into the simulation in order to permit a calculation of more than one rotation of the crankshaft. As we do not want to simulate the combustion process itself, but only need an initial point at the beginning of every scavenging according to the measured conditions, the second approach has been implemented.

\subsubsection{Exhaust Gas}

\section{The Stoichiometric Combustion}

In the setting of the two-stroke engine, we have to deal with air and exhaust gas, which are themselves mixtures of nitrogen $\mathrm{N}_{2}$, oxygen $\mathrm{O}_{2}$, hydrogen $\mathrm{H}_{2}$, carbon monoxide $\mathrm{CO}$, carbon dioxide $\mathrm{CO}_{2}$ and water steam $\mathrm{H}_{2} \mathrm{O}$. Obviously exhaust gas has a significantly different composition than air. The composition of the exhaust gas is mostly determined by the air-fuel ratio $A F R$ (see Definition 2.3.16 on page 15). The chemical equation of the stoichiometric combustion of octane with air in the case of a, mass based, $A F R$ of 15 is given by

$$
2 \mathrm{C}_{8} \mathrm{H}_{18}+25\left(\mathrm{O}_{2}+\frac{79}{21} \mathrm{~N}_{2}\right)=16 \mathrm{CO}_{2}+18 \mathrm{H}_{2} \mathrm{O}+\frac{1975}{21} \mathrm{~N}_{2} .
$$

The composition of the exhaust gas at stoichiometry is $73.45 \% \mathrm{~N}_{2}, 12.50 \% \mathrm{CO}_{2}$ and $14.05 \% \mathrm{H}_{2} \mathrm{O}$.

At a rich combustion $(A F R<15)$ carbon monoxide is also formed, whereas at lean combustion $(A F R>15)$ oxygen is additionally present in the exhaust gas. 


\section{Dissociation Effects}

In the hot, high-pressure conditions inside the cylinder during combustion effects of dissociation will also occur. This means that free carbon monoxide and free hydrogen are being created by two principal dissociation reactions:

$$
\begin{gathered}
\mathrm{CO}_{2} \rightleftharpoons \mathrm{CO}+\frac{1}{2} \mathrm{O}_{2} \\
\mathrm{H}_{2} \mathrm{O}+\mathrm{CO} \rightleftharpoons \mathrm{H}_{2}+\mathrm{CO}_{2}
\end{gathered}
$$

For the simulation of the combustion process itself the dissociation would have been taken into account, but after the combustion the expansion has cooled down the exhaust gas sufficiently, and dissociation effects can be neglected during the scavenging process (see also [B196]).

\subsection{Evaluation of Numerical Results}

\subsubsection{Characteristics}

There are three important characteristics in two-stroke engine design, the $S R-$ $S E$ characteristic, the $S R-T E$ characteristic, and the purity at exhaust port entry, which will be described below. (For the description of the scavenge ratio $S R$, the scavenge efficiency $S E$, and the trapping efficiency $T E$, refer to Section 2.3.2 on page 13.) As the hot exhaust gas has a significantly lower density than the entering fresh charge, it is also important to state whether the characteristics are mass related or volume related.

For diagrams derived from the numerical solution of the simulation of a twostroke engine, see Paragraph 10.6.5 on page 186.

\section{SR - SE Characteristic}

An example of this kind of characteristic is shown on the left-hand side of Figure 2.7.1 on the following page. As the scavenge efficiency is closely related to the purity of the trapped charge, this diagram gives a good impression of the combustibility of the trapped charge. At too low values of purity the engine starts misfiring. The disadvantage of this chart is the lower visual clarity for a comparison of the scavenging behavior.

\section{SR - TE Characteristic}

For a comparison of the scavenging process of two engines it is therefore more favorable to use the $S R-T E$ chart. Particularly the short-circuit (at low $S R$ levels) is more evident here. Also, there is a better quantitative judgment of the possible power potential. Figure 2.7.1 (middle) depicts a $S R-T E$ graph. 


\section{Purity at Exhaust Port Entry}

This is another possibility of quantitatively analyzing the difference between two engines (see Figure 2.7.1 (right)). A strong short-circuit at low loads is very well visible here. Towards the end of $S R$ range a low purity at exhaust port entry can be attributed to a low purity of the cylinder charge caused by significant losses during the first part of the scavenging process. Therefore, the more linear the profile, the worse the scavenging is as opposed to more " $\mathrm{S}$ "-like profiles.
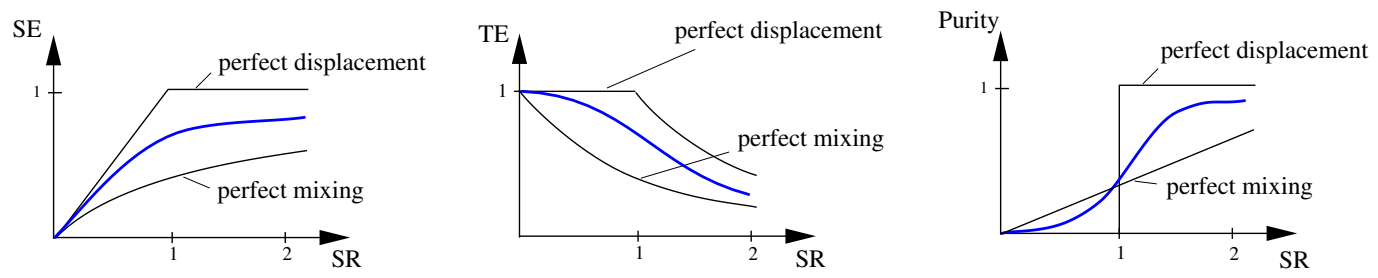

Figure 2.7.1: SR-SE (left), SR-TE (middle), and SR-purity at exhaust port entry (right) diagrams.

\subsubsection{Jante Test}

To derive a technique for measuring the scavenging efficiency in two-stroke engines, JANTE ([Jan68]) envisaged the following experiment. The cylinder in question is being motored at some constant speed with the cylinder head removed. The crankcase pumps the air through the transfer ports and the scavenging flow exits out of the cylinder head. In the upper part of the cylinder a comb of pitot tubes measures the vertical velocity contours. It has been shown that the results of the Jante testing technique correlates with the scavenging efficiency.

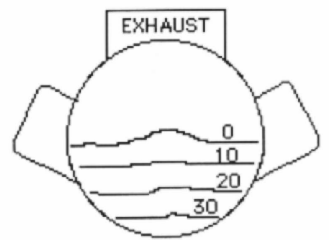

(A) SYMMETRICAL PATTERN

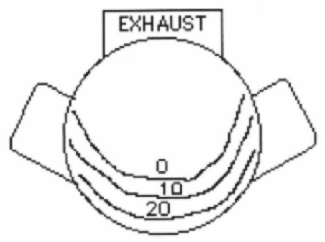

(C) WALL PATTERN

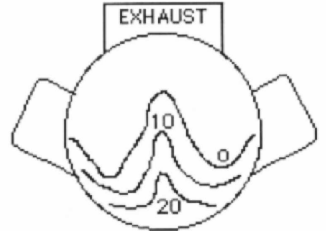

(B) TONGUE PATTERN

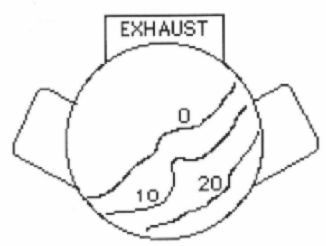

(D) UNSYMMETRICAL PATTERN

Figure 2.7.2: Different flow patterns according to JANTE (image taken from [B196]). 
JANTE identified several scavenging patterns for loop-scavenged engines (see Figure 2.7.2 on the preceding page). The symmetrical pattern (a) is the only one that yields good scavenging. In the "tongue" pattern (b), the fast flow in the center would reach the exhaust port before its closure, and in the "wall" pattern (c) and the asymmetrical pattern (d) the flow would enclose the exhaust gas and mix with it rather than displace it out of the exhaust duct.

This experimental setup and the resulting patterns can also be used to analyze the flow pattern resulting from a numerical CFD simulation, which obviously has the great advantage of a cylinder head in place (see also Paragraph 10.6.6 on page 190 for the results of the numerical simulation).

\subsubsection{Sharp vertical Velocity Boundary}

Another experimental finding of this type is the forming of the boundary between the rising and descending part of the flow during loop scavenging. In [B196, Chapter 3.4] it is stated that the sharper this boundary is, the better the scavenging characteristics of the cylinder. Hence, vertical plots of velocity and purity of charge (see Figure 2.7.3 and Paragraph 10.6.7 on page 190) can reveal this boundary and help to analyze the flow situation in the cylinder.

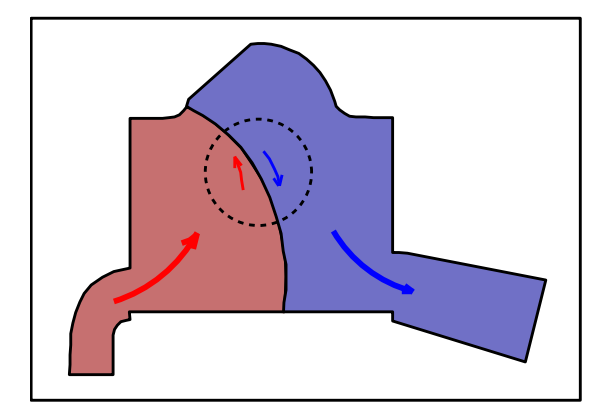

Figure 2.7.3: Vertical velocity boundary.

\subsubsection{Deviation of the Flow Direction from the Transfer Port Ori- entation}

The transfer port orientation is vital for directing the incoming flow and providing good scavenging (see also Section 2.4.1 on page 15). But the flow does not follow these angles exactly. Instead, there exists a deviation from the design direction by an angle $\alpha_{\mathrm{dh}}$ in the horizontal plane and an angle $\alpha_{\mathrm{dv}}$ vertically (see Figure 2.7.4 on the following page), which furthermore varies from port opening to bdc. In computational simulations this deviation is easily detectable and its effects can be studied (as is done in Paragraph 10.6.8 on page 190). 

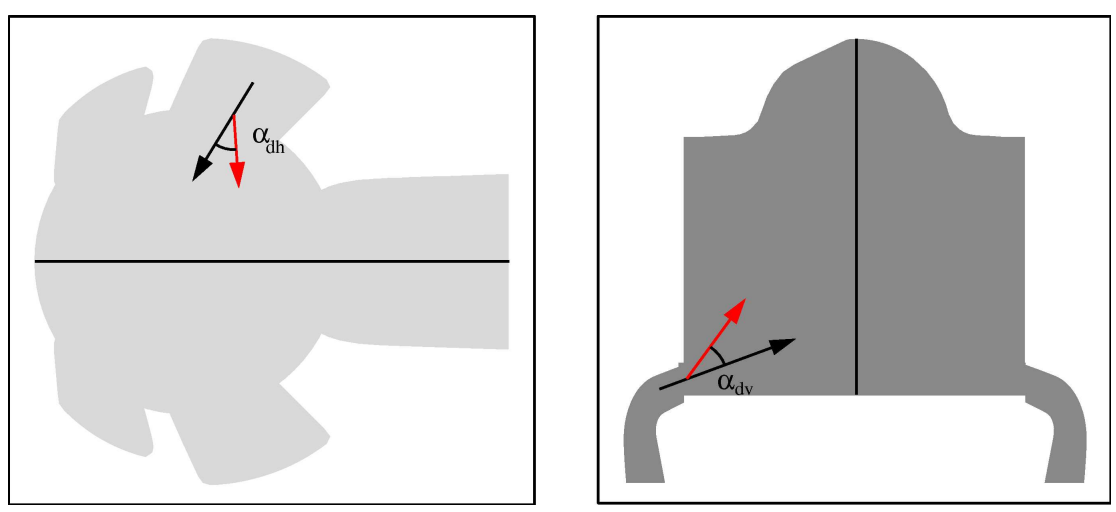

Figure 2.7.4: Deviation of the real flow angle (horizontal deviation angle $\alpha_{d h}$ and vertical deviation angle $\alpha_{d v}$ ).

\subsubsection{Squish Velocity}

Squishing occurs at the end of the upward motion of the piston, when the gas is forced (squished) from the narrow fringe of the cylinder head towards the center of the combustion chamber. A good squish action assists in good combustion characteristics and lowers the risk of detonation at high compression ratios due to an even distribution of fresh charge. The visualization of the squish velocities in the combustion chamber prior to ignition is one possibility to get an insight of the squishing action of an engine cylinder. Figure 2.7.5 shows the averaged horizontal part of the velocities starting from outlet closure until tdc. The results of the numerical calculations regarding the squish velocity are presented in Paragraph 10.6.9 on page 191.

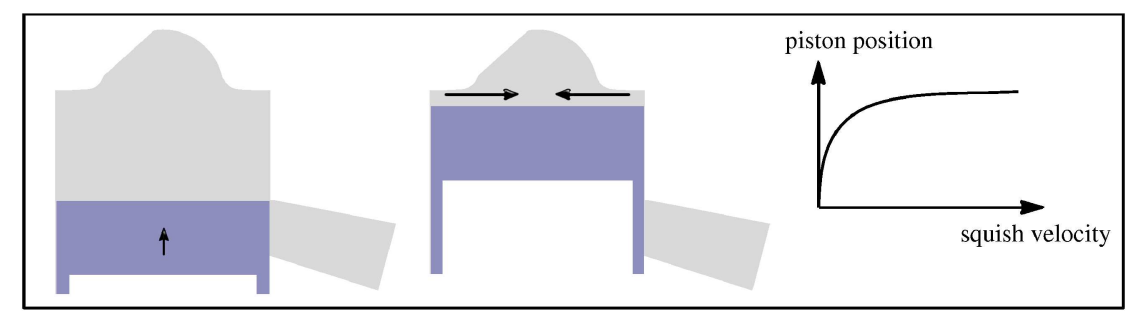

Figure 2.7.5: Piston at closure of exhaust port (left), at tdc (middle) and squish velocity (right). 


\section{Chapter 3}

\section{Physical Background}

\subsection{Definition of the Determining Variables of a Fluid}

The state of a system is given, if the values of certain parameters, which completely determine all relevant characteristics of the system, are known. By saying this, it is not meant that one has to know at every instant of time the position and motions of all the particles constituting the system, but rather macroscopic parameters have to be taken into consideration. Macroscopic parameters are statistical averages of a large number of molecules. For example, the macroscopic velocity $\mathbf{v}$ may be introduced as the velocity of the center of gravity of the molecules in a volume, or the pressure $p$ acting on a plane as the mean characteristic impulse of the molecules acting on this plane during their random motion (see also [Sed72]).

The temperature $T$ of a system is given as a result of the "Zeroth Law of Thermodynamics". Two systems in thermal equilibrium have, by definition, the same temperature. Whereas systems that are not in thermal equilibrium have different temperatures. In order to fix a temperature scale the equation of state of an ideal gas is employed (see Section 3.5 on page 36). The absolute temperature in Kelvin results from the equation of state for a perfect gas $T=\frac{p V}{n \mathcal{R}}$ with pressure $p$, volume $V$, total number of moles of molecules $n$ and universal gas constant $\mathcal{R}$ (cf. Section 3.5 on page 36 and [AH92]).

The density of a fluid is defined as $\rho(\mathbf{x}):=\lim _{\varepsilon \rightarrow 0} \frac{\Delta m}{B_{\varepsilon}(\mathbf{x})}$ with mass $\Delta m$ of the ball $B_{\varepsilon}(\mathbf{x})$ around $\mathbf{x}$ with radius $\varepsilon$. As opposed to liquids, gases fill a given volume while changing their density. But even in gases the density changes are small if the flow of the gas is slow compared to the speed of sound in that gas. However, in our application of the two-stroke engine a Mach number of 1 is approached by the flow in various locations. As a consequence, a compressible fluid has to be assumed.

The First Law of Thermodynamics states that the specific (i.e. per unit mass) internal energy $\varepsilon$ is defined (up to a constant $\varepsilon_{0}$ ) by the total (exact) differential (see e.g. [AH92])

$$
d \varepsilon:=d a+d q,
$$

where $d a$ and $d q$ are the change of specific work and specific heat respectively. ${ }^{1}$ This means that $\varepsilon$ is a state function: $\int_{S_{1}}^{S_{2}} d \varepsilon$ depends only on the integration bounds

\footnotetext{
${ }^{1} \overline{d a \text { and } d q \text { are not total differentials. Work } a}$ and heat $q$ are process functions not state functions, their changes depend on the path from state 1 to state 2 (e.g. reversible vs. irreversible changes).
} 
leading to $\int_{S_{1}}^{S_{2}} d \varepsilon=\varepsilon\left(S_{2}\right)-\varepsilon\left(S_{1}\right)$, no matter on which path we reach state $S_{2}$ from state $S_{1}$. Physically speaking, only the difference of the internal energy in two states is important, so the constant $\varepsilon_{0}$ is not relevant. The specific total energy is then the sum of internal and kinetic energy: $E:=\varepsilon+\frac{|v|^{2}}{2}$ and the total energy density is $e:=\rho E$.

All of the above-stated characteristic (thermodynamic) variables of a fluid are functions of space and time: $\rho=\rho(\mathbf{x}, t), \mathbf{v}=\mathbf{v}(\mathbf{x}, t), E=E(\mathbf{x}, t), e=e(\mathbf{x}, t)$, $p=p(\mathbf{x}, t), T=T(\mathbf{x}, t)$.

For further details see e.g. [LL95].

\subsection{The Material Properties}

\subsubsection{The Heat Capacities $\mathrm{c}_{\mathrm{p}}$ and $\mathrm{c}_{\mathrm{V}}$}

The heat capacity $C$ is defined by the amount of heat which must be supplied to a medium in order to raise its temperature by $1 K$. It is an extensive quantity ( $100 \mathrm{~g}$ of water has a heat capacity 100 times bigger than $1 g$ of water). $c:=\frac{C}{n M}$ with molecular weight $M$ and total number of moles of molecules $n$ is the intensive (or specific) heat capacity. The unit of the specific heat capacity is $\frac{\mathrm{J}}{\mathrm{kg} \mathrm{K}}=\frac{\mathrm{m}^{2}}{\mathrm{~s}^{2} \mathrm{~K}}$. We have the following

Definition 3.2.1. (specific heat capacity) The specific heat capacity is defined as

$$
c:=\frac{d q}{d T}
$$

with $d q$ the heat flux per unit mass of the fluid.

If the total work $d a$ done on the system, or by the system, is only volumetric work $d a=-p d v$, with change in volume $d v$, equation (3.1.1) can be written as

$$
d q=d \varepsilon+p d v .
$$

With the specific enthalpy $h:=\varepsilon+p v$, because of $d h=d \varepsilon+p d v+v d p$,

$$
d q=d h-v d p
$$

also follows. ( $h$ is a state function (and thus $d h$ is an exact differential) because $\varepsilon$, $p$ and $v$ are state functions.) From (3.2.1), (3.2.2), and (3.2.3) we have

$$
c=\frac{d \varepsilon}{d T}+p \frac{d v}{d T}=\frac{d h}{d T}+v \frac{d p}{d T} .
$$

Definition 3.2.2. (specific heat capacity at constant volume and specific heat capacity at constant pressure) Therefore, at constant volume (isochore process, $d v=0$ ) one gets from equation (3.2.4)

$$
c_{\mathrm{V}}:=\frac{\partial \varepsilon}{\partial T} .
$$

Similarly for constant pressure (isobaric process, $d p=0$ ) one has

$$
c_{p}:=\frac{\partial h}{\partial T} .
$$


In the situation of an ideal gas (see Section 3.5 on page 36 ) one can show that $\varepsilon$ and $h$ are functions of $T$ only: $\varepsilon=\varepsilon(T)$ and $h=h(T)$.

The unit of the specific heat capacity is $\frac{\mathrm{J}}{\mathrm{kg} \mathrm{K}}=\frac{\mathrm{m}^{2}}{\mathrm{~s}^{2} \mathrm{~K}}$.

There exists a further link between the two heat capacities $c_{\mathrm{V}}$ and $c_{p}$ in the case of the ideal gas ${ }^{2}$ :

$$
(d q)_{p}=d \varepsilon+p d v=c_{\mathrm{V}} d T+d(p v)=\left(c_{\mathrm{V}}+R\right) d T,
$$

where we have used the ideal gas law $p v=R T$ (see Section 3.5 on page 36) in the last equation. We have as a result

$$
c_{p}-c_{\mathrm{V}}=R,
$$

which is known as Mayer's formula.

\subsubsection{The Adiabatic Exponent $\gamma$}

A process is said to be adiabatic if the heat flux vanishes: $d q=0$. In a reversible expansion or compression the volumetric work is given by $d a=-p d v$. From (3.1.1) and the definition of $c_{\mathrm{V}}$ (3.2.5) follows that the change of internal energy is

$$
d \varepsilon=-p d v=c_{\mathrm{V}} d T .
$$

Using the equation of state of a thermally perfect gas (3.5.1), we deduce

$$
-\frac{R T}{V} d V=c_{\mathrm{V}} d T .
$$

Assuming also a calorically perfect gas (see Section 3.5 on page 36 ), the heat capacity is constant $c_{\mathrm{V}}(T) \equiv c_{\mathrm{V}}$, and we get by integration

$$
-R \ln \left(\frac{V_{1}}{V_{0}}\right)=c_{\mathrm{V}} \ln \left(\frac{T_{1}}{T_{0}}\right) .
$$

Since the ln-function is monotone and by using the thermal equation of state again one obtains

$$
\left(\frac{V_{0}}{V_{1}}\right)^{\frac{R}{c_{\mathrm{V}}}}=\frac{p_{1} V_{1}}{p_{0} V_{0}}
$$

which is equivalent to

$$
\left(\frac{V_{0}}{V_{1}}\right)^{\gamma}=\frac{p_{1}}{p_{0}}
$$

with

$$
\gamma:=\frac{R}{c_{\mathrm{V}}}+1=\frac{c_{p}}{c_{\mathrm{V}}} .
$$

Thus, $\gamma$ governs the pressure change in reversible adiabatic expansions or compressions of a perfect gas and is therefore called adiabatic exponent. In the case of a calorically imperfect gas $\left(c_{\mathrm{V}}=c_{\mathrm{V}}(T)\right.$ and $\left.c_{p}=c_{p}(T)\right)$ the definition of gamma is retained:

Definition 3.2.3. (adiabatic exponent) The adiabatic exponent is defined as

$$
\gamma(T):=\frac{c_{p}(T)}{c_{\mathrm{V}}(T)}
$$

\footnotetext{
${ }^{2}$ Where the index $p$ indicates a process at constant pressure.
} 


\subsubsection{The Speed of Sound c}

The speed of sound $c$ can be calculated as (see e.g. [GR96], [Sed72])

$$
c^{2}=\gamma(T) R T=\frac{\gamma(T) p}{\rho} .
$$

Since pressure, density, and temperature usually depend on space and time the speed of sound $c$ of a fluid is not a constant physical quantity.

\subsubsection{The Viscosity $\eta$}

Definition 3.2.4. (dynamic viscosity) For Newtonian fluids ${ }^{3}$, Newtons elementary law of shear viscosity states that the shear stress $\tau$ is defined by $\tau=\eta \frac{\partial u}{\partial y}$ with the dynamic (shear) viscosity $\eta$ and the velocity change normal to the contact face $\frac{\partial u}{\partial y}$.

The unit of the dynamic viscosity is $\frac{\mathrm{N} \mathrm{s}}{\mathrm{m}^{2}}=\frac{\mathrm{kg}}{\mathrm{sm}}$. This dynamic shear viscosity is again encountered at the derivation of the Navier-Stokes equations (see Section 3.3.2 on page 32) alongside with the bulk viscosity $\widehat{\eta}$. This bulk viscosity is not known very precisely up to now, but experience seems to confirm the hypotheses $\widehat{\eta}=0$, which was already proposed by Stokes (cf. [Tru89, page 123]).

\subsubsection{The Thermal Conductivity $\lambda$}

The heat flux between two neighboring fluid elements is proportional to the temperature difference between these two elements. The heat flux $\Delta \Phi$ through the contact area $\Delta A$ is defined as $\Delta \Phi=\varphi \Delta A$ with the thermal flux density $\varphi$.

Definition 3.2.5. (Thermal conductivity) Fourier's law states that the thermal flux density is given by $\varphi=-\lambda \frac{\partial T}{\partial y}$ with the thermal conductivity $\lambda$ and the temperature change normal to the contact face $\frac{\partial T}{\partial y}$. The unit of the thermal conductivity is $\frac{\mathrm{N}}{\mathrm{sK}}=\frac{\mathrm{J}}{\mathrm{s} \mathrm{Km}}=\frac{\mathrm{W}}{\mathrm{Km}}$.

For further details on the derivation of heat capacity, adiabatic exponent, viscosity and thermal conductivity refer to [AH92, AdP01, Sed72, Tru89].

The heat capacity, like the viscosity and the thermal conductivity, is temperaturedependent, which has to be considered for fluids with large temperature differences (see Section 3.8 on page 40).

\subsubsection{The Reynolds and Prandtl Numbers}

Fluid flows can be regarded as similar in some sense. In the Navier-Stokes equations we only have the viscosity $\eta$ and the thermal diffusivity $\alpha:=\frac{\lambda}{\rho c_{p}}$ as characterizing parameters of the fluid. If the shape of the domain of two different flow regimes is the same then we can construct two dimensionless numbers

\footnotetext{
${ }^{3}$ Non-Newtonian fluids are, for example, visco-inelastic fluids where the shear stress does not depend linearly on $\frac{\partial u}{\partial y}$, or time-dependent shear stress laws where the viscosity also depends on the duration of stress, $\tau=f\left(\frac{\partial u}{\partial y}, t\right)$, so-called "memory materials".
} 


$$
\begin{aligned}
\operatorname{Re} & :=\frac{\rho_{0} v_{0} L_{0}}{\eta_{0}} \text { and } \\
\operatorname{Pr} & :=\frac{c_{p_{0}} \eta_{0}}{\lambda_{0}}
\end{aligned}
$$

with characteristic values for the density of the fluid $\rho_{0}$, the velocity $v_{0}$, size of the geometry $L_{0}$, the viscosity $\eta_{0}$, the heat capacity at constant pressure $c_{p_{0}}$, and the thermal conductivity $\lambda_{0}$. If the two flow regimes have the same Reynolds and Prandtl numbers then they are considered similar. The same fluid phenomena can be observed in the two flows, just in different scales.

\subsection{The Navier-Stokes Equations}

Now that we have collected all the necessary variables, we can proceed to state the fundamental systems of partial differential equations that describe a compressible fluid.

\subsubsection{The Euler Equations}

From the conservation of mass, momentum and energy of a compressible inviscid fluid (where we neglect heat conduction) the Euler equations of gas dynamics can be derived (see also [FP97, Tru89]). In Eulerian coordinates they can be written in the following conservative form:

$$
\frac{\partial \mathbf{U}}{\partial t}+\sum_{j=1}^{3} \frac{\partial}{\partial x_{j}} \mathbf{f}_{j}(\mathbf{U})=\mathbf{0}, \quad \mathbf{x}=\left(x_{1}, x_{2}, x_{3}\right) \in \mathbb{R}^{3}, \quad t>0,
$$

where the vector of conservative variables is

$$
\mathbf{U}=\left(\begin{array}{c}
\rho \\
\rho v_{1} \\
\rho v_{2} \\
\rho v_{3} \\
e
\end{array}\right), \quad e=\rho E
$$

with $\mathbf{U}$ taking values in the set of states

$$
\Psi=\left\{\left(\rho, \rho \mathbf{v}=\left(\rho v_{1}, \rho v_{2}, \rho v_{3}\right), e\right) ; \rho>0, \mathbf{v} \in \mathbb{R}^{3}, e-\frac{\rho}{2}|\mathbf{v}|^{2}>0\right\},
$$

and the convective flux functions

$$
\mathbf{f}_{1}(\mathbf{U})=\left(\begin{array}{c}
\rho v_{1} \\
\rho v_{1}^{2}+p \\
\rho v_{1} v_{2} \\
\rho v_{1} v_{3} \\
(e+p) v_{1}
\end{array}\right), \quad \mathbf{f}_{2}(\mathbf{U})=\left(\begin{array}{c}
\rho v_{2} \\
\rho v_{1} v_{2} \\
\rho v_{2}^{2}+p \\
\rho v_{2} v_{3} \\
(e+p) v_{2}
\end{array}\right), \quad \mathbf{f}_{3}(\mathbf{U})=\left(\begin{array}{c}
\rho v_{3} \\
\rho v_{1} v_{3} \\
\rho v_{2} v_{3} \\
\rho v_{3}^{2}+p \\
(e+p) v_{3}
\end{array}\right) .
$$


In order to close the system an additional equation of state for the pressure $p$ has to be provided (see Section 3.5 on page 36 ).

The Euler equations are a hyperbolic symmetrizable nonlinear system of conservation laws (refer to [GR96]).

\subsubsection{The Navier-Stokes Equations}

If we also take the viscosity and the thermal conductivity of the fluid into account, the Euler equations (3.3.1) are replaced by the Navier-Stokes equations:

$$
\frac{\partial \mathbf{U}}{\partial t}+\sum_{j=1}^{3} \frac{\partial}{\partial x_{j}} \mathbf{f}_{j}(\mathbf{U})-\sum_{j=1}^{3} \frac{\partial}{\partial x_{j}} \mathbf{h}_{j}=\mathbf{0}, \quad \mathbf{x}=\left(x_{1}, x_{2}, x_{3}\right) \in \mathbb{R}^{3}, \quad t>0,
$$

with $\mathbf{U}$ and the convective flux functions $\mathbf{f}_{j}$ as in (3.3.1), and the viscous flux functions

$$
\mathbf{h}_{j}\left(\mathbf{U}, \frac{\partial \mathbf{U}}{\partial x_{1}}, \frac{\partial \mathbf{U}}{\partial x_{2}}, \frac{\partial \mathbf{U}}{\partial x_{3}}\right)=\left(\begin{array}{c}
0 \\
\tau_{j 1} \\
\tau_{j 2} \\
\tau_{j 3} \\
\sum_{l=1}^{3} \tau_{j l} v_{l}+\lambda \frac{\partial T}{\partial x_{j}}
\end{array}\right), \quad 1 \leq j \leq 3
$$

with

$$
\begin{gathered}
\tau_{i j}=2 \eta D_{i j}(\mathbf{U})-\frac{2}{3} \eta \delta_{i j} \operatorname{div} \mathbf{v}, \\
D_{i j}(\mathbf{U})=\frac{1}{2}\left(\frac{\partial v_{i}}{\partial x_{j}}+\frac{\partial v_{j}}{\partial x_{i}}\right),
\end{gathered}
$$

the deformation tensor $D(\mathbf{U}):=\left(D_{i j}(\mathbf{U})\right)$, the temperature $T$, the dynamic shear viscosity $\eta$, and the thermal conductivity $\lambda$. As mentioned in Paragraph 3.2.4 on page 30 the bulk viscosity $\hat{\eta}$ is here set to zero.

To close the system (3.3.4) it is necessary to add two equations of state for $p$ and $T$ (see Section 3.5 on page 36 ).

\subsection{Initial Conditions and Boundary Conditions}

The time interval $\mathcal{T}$ and the region $\Omega$, in which the flow problem is considered, are described next.

\subsubsection{Initial Conditions}

We want to observe the temporal development of the flow. Therefore, we have to start at an initial instant $T_{\text {start }}:=0$. The physical problem that describes this procedure is given by the following

Definition 3.4.1. (Initial value problem) Let $\mathbf{U}, \mathbf{f}_{j}$, and $\mathbf{h}_{j}$ be defined as in Section 3.3 on the preceding page. Then the following problem is called initial value problem or Cauchy problem: 
Find a weak entropy solution $\mathbf{U}(\mathbf{x}, t)$ such that for $T_{\mathrm{end}} \in \mathbb{R}^{+}$

$$
\left\{\begin{aligned}
\frac{\partial}{\partial t} \mathbf{U}+\sum_{i=1}^{3} \frac{\partial}{\partial x_{i}} \mathbf{f}_{i}(\mathbf{U})+\sum_{i=1}^{3} \frac{\partial}{\partial x_{i}} \mathbf{h}_{i}(\mathbf{U}) & =\mathbf{0} & & \text { in } \mathbb{R}^{3} \times\left(0, T_{\text {end }}\right) \\
\mathbf{U}(\mathbf{x}, 0) & =\mathbf{U}_{0}(\mathbf{x}) & & \text { in } \mathbb{R}^{3}
\end{aligned}\right.
$$

with initial conditions $\mathbf{U}_{0} \in L^{\infty}(\Omega)$ given.

Remark 3.4.2. With the help of characteristics it can be shown that in general there exists no classical solution of (3.4.2). The concept of weak solutions has to be introduced. These are unique only if they satisfy an additional property, the entropy condition. For further details refer to [GR96] and [Krö97].

\subsubsection{Boundary Conditions}

If we have an open finite region $\Omega \subset \mathbb{R}^{3}$, which is occupied by the medium under consideration, additional boundary conditions have to be specified.

Definition 3.4.3. (Initial boundary value problem) Let $\mathbf{U}, \mathbf{f}_{j}$, and $\mathbf{h}_{j}$ be defined as in Section 3.3 on page 31. Then the initial boundary value problem can be stated as:

Find a weak entropy solution $\mathbf{U}(\mathbf{x}, t)$ such that for open finite $\Omega \subset \mathbb{R}^{3}$ and $T_{\text {end }} \in \mathbb{R}^{+}$

$$
\left\{\begin{aligned}
\frac{\partial}{\partial t} \mathbf{U}+\sum_{i=1}^{3} \frac{\partial}{\partial x_{i}} \mathbf{f}_{i}(\mathbf{U})+\sum_{i=1}^{3} \frac{\partial}{\partial x_{i}} \mathbf{h}_{i}(\mathbf{U}) & =\mathbf{0} & & \text { in } \Omega \times\left(0, T_{\text {end }}\right) \\
\mathbf{U}(\mathbf{x}, 0) & =\mathbf{U}_{0}(\mathbf{x}) & & \text { in } \Omega \\
\mathbf{U}(\mathbf{x}, t) & =\mathbf{g}(\mathbf{x}, t) & & \text { on } \Gamma \subseteq \partial \Omega \times\left(0, T_{\text {end }}\right)
\end{aligned}\right.
$$

with the initial conditions $\mathbf{U}_{0} \in L^{\infty}(\Omega)$ and the boundary conditions $\mathbf{g} \in$ $L^{\infty}\left(\partial \Omega \times\left(0, T_{\text {end }}\right)\right)$ given.

\section{Existence and Uniqueness for the Initial Boundary Value Problem in the Scalar Case}

For a well-posed initial boundary value problem the boundary conditions cannot be chosen arbitrarily. They depend strongly on the problem in question and the initial conditions. This can be seen by considering the following one-dimensional scalar linear model problem (here we follow [Krö97]):

$$
\begin{aligned}
\frac{\partial}{\partial t} u(x, t)+a \frac{\partial}{\partial x} u(x, t) & =0 & & \text { in } \mathbb{R}^{+} \times \mathbb{R}^{+} \\
u(x, 0) & =u_{0}(x) & & \text { in } \mathbb{R}^{+}
\end{aligned}
$$

Boundary conditions $u(0, t)=g(t)$ can only be imposed if $a>0$. Otherwise the initial data $u_{0}(x)$ is advected to the boundary and by imposing boundary conditions the problem is, in general, over-determined. But in any case the condition

$$
\left.\min _{k \in I(\gamma u, g)}\{-a \cdot|\gamma u-k|\}=0 \quad \text { (pointwise in }(x, t) \in \mathbb{R}^{+} \times \mathbb{R}^{+}\right),
$$


with the trace operator $\gamma$, is satisfied (for the definition of such a trace operator $\gamma$ and the requirements on $u$ for the existence of $\gamma$ cf. [BLN79, Krö97, Ott96]). Here we have used the notation

$$
I(a, b):=[\min \{a, b\}, \max \{a, b\}] .
$$

If $a>0$ the minimum is equal to zero if and only if $\gamma u=g$, i.e. $u$ assumes the prescribed boundary values. For $a<0$ the minimum is obtained at $k=\gamma u$ independent of $g$ and is equal to zero.

The generalization to the nonlinear equation with homogeneous boundary condition $a=0$ is

$$
\min _{k \in I(\gamma u, 0)}\{\operatorname{sign}(\gamma u)(f(\gamma u)-f(k)) \cdot n\}=0 .
$$

Thus, we consider the following initial boundary value problem:

$$
\begin{aligned}
\frac{\partial}{\partial t} u+\operatorname{div} f(u) & =0 & & \text { in } \Omega \times(0, T) \\
u(x, 0) & =u_{0}(x) & & \text { in } \Omega \\
\min _{k \in I(\gamma u, 0)}\{\operatorname{sign}(\gamma u)(f(\gamma u)-f(k)) \cdot n\} & =0 & &
\end{aligned}
$$

For the next definition and the existence and uniqueness theorem we follow [BLN79]. A corresponding theorem for $u \in L^{\infty}$ is given in [Ott96].

Definition 3.4.4. (Weak solution of the initial boundary value problem) A function $u \in B V(\Omega \times(0, T))$ is a weak solution of (3.4.3) if for all $k \in \mathbb{R}$ and all non-negative test functions $\varphi \in C_{0}^{2}(\bar{\Omega} \times(0, T))$ with compact support in $\bar{\Omega} \times(0, T)$, it satisfies the following inequality

$$
\begin{aligned}
\int_{\Omega \times(0, T)}(|u-k| & \left.\frac{\partial}{\partial t} \varphi+\operatorname{sign}(u-k)(f(u)-f(k)) \nabla \varphi\right) \\
& +\int_{0}^{T} \int_{\partial \Omega} \operatorname{sign}(k)(f(\gamma u)-f(k)) \cdot n \varphi \geq 0
\end{aligned}
$$

and the initial condition

$$
u(x, 0)=u_{0}(x) \quad \text { in } \Omega
$$

almost everywhere in $\Omega$.

Theorem 3.4.5. (Existence and uniqueness for the initial boundary value problem) The initial boundary value problem (3.4.3) admits a unique solution in the sense of Definition 3.4.4 which is given by the vanishing viscosity method.

Proof. For the proof we refer to [BLN79].

\section{The Different Types of Boundary Conditions}

It follows a description of the different physical boundary conditions which are encountered in our problem. These boundary conditions arise mainly from physical considerations as no practical rules have emerged so far from mathematical investigations concerning existence theorems of the initial boundary value problem of the Navier-Stokes equations. 
But first the notion of Dirichlet and Von Neumann conditions has to be introduced. The specification of an explicit value at the boundary is said to be a Dirichlet boundary condition. For example, if the velocity on a solid wall is imposed: $v=0$. Whereas with a Von Neumann boundary condition a normal derivative is prescribed. For example, the heat flux at an adiabatic wall: $\frac{\partial T}{\partial n}=0$.

- Inflow boundary: This is an artificial boundary condition, since the real fluid extends beyond this boundary. But as the calculation domain has to be finite, this boundary, where the flow enters the numerical domain, is introduced.

The boundary conditions are generally determined by the physical experiment.

- Outflow boundary: The same is true for the outflow boundary condition where the flow leaves the numerical domain.

- Solid slip wall: The normal velocity of the fluid at the wall is equal to the velocity of the wall in normal direction: $\mathbf{v} \cdot \mathbf{n}=\mathbf{v}_{\text {wall }} \cdot \mathbf{n}$ with $\mathbf{n}$ the normal of the wall. Therefore, we impose a zero mass flux at this wall.

Different types of temperature treatment are possible:

- adiabatic boundary condition: There is no heat flux present between wall and fluid: $\frac{\partial T}{\partial n}=0$.

- isothermal boundary condition: The temperature of the wall is $T_{\text {wall }}$. This temperature is imposed as boundary condition: $T=T_{\text {wall }}$.

- Solid no-slip wall: Here also the tangential velocity component of the fluid is determined. Thus, the velocity of the fluid at the wall is equal to the velocity of the wall: $\mathbf{v}=\mathbf{v}_{\text {wall }}$. Also, with this wall condition no mass flux is possible. Because of the induced friction at the wall a boundary layer appears, where strong gradients of velocity and temperature are present (cf. [SG97]).

Concerning the temperature, the same adiabatic or isothermal condition as in the case of the slip wall can be applied.

- Symmetry boundary: This is also an artificial boundary condition. It is used mainly for saving computational time by cutting the numerical domain in half and assuming a symmetric flow pattern. Thus, the flow has to be symmetric at this boundary which implies that the normal component of the velocity vanishes: $\mathbf{v} \cdot \mathbf{n}=0$.

For all other variables of the flow Von Neumann conditions of zero flux apply.

Remark 3.4.6. As inflow boundary condition we use a massflow condition in our simulation of the two-stroke engine. The reason being the better comparability of the results. At different geometrical configurations we can ensure that with identical massflow through the engine the scavenge ratio $S R$ (see Paragraph 2.3.2 on page 13 for the definition) is also identical, thus, the trapping efficiency $T E$ of the configurations is directly comparable.

Remark 3.4.7. In our problem of simulating a two-stroke engine, after a few rotations of the crankshaft the flow behavior is mainly determined by the boundary 
conditions. The initial conditions act as a perturbation which decays asymptotically. In order to have a rapid convergence in time it is favorable to choose the initial conditions as close as possible to the real solution. We achieve this by an iterative approach described in Section 6.3 on page 97.

Remark 3.4.8. Symmetry boundary conditions are not unproblematic when simulating a viscous flow. Even at perfect symmetric conditions a completely unsymmetrical flow pattern can emerge as e.g. in the case of the Karman vortex street, or, at higher Reynolds numbers, in the attempt to capture the larger turbulent structures of the flow. Thus, careful testing has to be conducted before using this kind of boundary condition.

Remark 3.4.9. We are not considering the case of a boundary whose position is influenced by the flow itself. The piston motion, e.g., is not driven by the actual pressure difference between cylinder and crankcase but is prescribed by the explicit formula in Lemma 2.3.10 on page 13 .

For the details concerning the numerical implementation, refer to Section 4.7 on page 55 .

\subsection{The Equations of State}

\subsubsection{The Thermal Equation of State}

An ideal gas satisfies the laws of CHARLES and GAY-LussaC $\left(\frac{V}{T}=\right.$ const with $n$, $p=$ const), Boyle-Mariotte ( $p V=$ const with $n, T=$ const $)$, and the Avogadro principle $\left(\frac{V}{n}=\right.$ const with $T, p=$ const), where $V$ is the volume, $T$ the temperature, $n$ the total number of moles of molecules, and $p$ the pressure of the gas. From these laws the ideal gas law or thermal equation of state $p V=n \mathcal{R} T$ can be deduced. $\mathcal{R}$ is the (molar) universal gas constant $\mathcal{R}$ (for its value see Table B.1 on page 209). With the specific gas constant $R=\frac{\mathcal{R}}{M}$ in $\frac{\mathrm{J}}{\mathrm{kg} \mathrm{K}}$ ( $M$ being the molecular weight of the gas under consideration) and the density $\rho=\frac{1}{\tau}$ with the specific volume $\tau=\frac{V}{n M}$ the thermal equation of state can be written as

$$
p=\rho R T
$$

One says that the ideal gas is thermally perfect (or perfect).

\subsubsection{The Caloric Equation of State}

As stated in Section 3.2.1 on page 28 the specific heat capacity $c_{\mathrm{V}}(T)$ is defined by $c_{\mathrm{V}}(T)=\frac{d \varepsilon}{d T}$ or $d \varepsilon=c_{\mathrm{V}}(T) d T$ with specific internal energy $\varepsilon$. By integrating the last equation one obtains

$$
\varepsilon=\int_{T_{0}}^{T} c_{\mathrm{V}}(\tau) d \tau+\varepsilon_{0}
$$


with constants $T_{0}$ and $\varepsilon_{0}$. As mentioned earlier (see Section 3.1 on page 27) these constants are physically not of interest, one can set $T_{0}=0$ and $\varepsilon_{0}=0$. The resulting caloric equation of state for a calorically imperfect gas is thus given by

$$
\varepsilon=\int_{0}^{T} c_{\mathrm{V}}(\tau) d \tau
$$

For a calorically perfect gas, also called polytropic gas, $c_{\mathrm{V}}$ is independent of the temperature $T$, the caloric equation of state can then be stated as

$$
\varepsilon=c_{\mathrm{V}} T \text {. }
$$

An equivalent formulation is given with help of (3.2.8) and (3.5.1):

$$
p=(\gamma-1) \rho \varepsilon
$$

\subsection{Ideal Gas - Real Gas}

The ideal gas law (3.5.1) is derived by neglecting the intermolecular forces. These forces are only important when the molecules of a gas are relatively close together. Therefore, the ideal gas law is exact in the limit $p \rightarrow 0$.

Definition 3.6.1. (compression factor) The deviation of the behavior of a gas from this ideal gas law is measured by the compression factor

$$
Z:=\frac{p}{\rho R T} .
$$

The regime in our application of the two-stroke engine reaches a maximum pressure of about 25 bar in the compression cycle inside the cylinder. The compression factor for air at this pressure is 1.009 (cf. [Vdi97]). So for our needs the ideal gas law is exact up to one percent. No real gas effects have to be taken into account. No other thermal equation of state, e.g. the VAN DER WAALS equation, has to be used.

Furthermore, condensation does not have to be considered. The critical temperature $T_{c}$ and critical pressure $p_{c}$ of air (see Appendix B on page 209) are far beyond the range of our data.

\subsection{Gas Mixtures}

\subsubsection{Notations}

In a multi-component system of $N$ different species the total number of moles is given by

$$
n_{m}:=\sum_{i=1}^{N} n_{i}
$$

with $n_{i}$ being the the number of moles of each species. Hence, the mole fractions are

$$
y_{i}:=\frac{n_{i}}{n_{m}}, \quad i=1,2, \ldots, N \quad \text { with } \quad \sum_{i=1}^{N} y_{i}=1 .
$$


The total mass of the mixture is similarly defined as

$$
m_{m}:=\sum_{i=1}^{N} m_{i}
$$

where $m_{i}:=M_{i} n_{i}$ is the mass of species $i$ and $M_{i}$ the components' molecular weight. Therefore, the mass fractions are

$$
z_{i}:=\frac{m_{i}}{m_{m}}, \quad i=1,2, \ldots, N \quad \text { with } \quad \sum_{i=1}^{N} z_{i}=1 .
$$

\subsubsection{The Equations of State}

In the case of non-reacting mixtures of gases Dalton's law states that

$$
p=\sum_{i} p_{i},
$$

the total pressure is the sum of the partial pressures of each gas in the mixture. With this result the thermal equation of state (3.5.1) becomes

$$
p=\sum_{i} y_{i} \rho \mathcal{R} T
$$

with $y_{i}$ the mole fraction of component $i$.

The heat capacity $c_{v m}$ of a mixture of ideal gases follows the exact mixing formula (see Section 3.8 on page 40)

$$
c_{v m}=\sum_{i} z_{i} c_{v i}
$$

with the heat capacity $c_{v i}$ of component $i$. Therefore, the caloric equation of state (3.5.2) is transformed to

$$
\varepsilon=\int_{0}^{T} \sum_{i} z_{i} c_{v i}(\tau) d \tau .
$$

For the composition of the exhaust gas used in the simulation refer to Paragraph 2.6.3 on page 22 .

Remark 3.7.1. We have stated in Section 3.1 on page 27 that the constant $\varepsilon_{0}$ is not of physical interest. In our case, where the different gases do not interact, this remains true for gas mixtures. The constant $\varepsilon_{0}$ would be, e.g., necessary for reacting gases or phase transitions.

\subsubsection{Fresh Gas, Exhaust Gas}

In our application of the two-stroke engine we use the aforementioned gas mixture formulas for modeling the fresh gas, assumed to have the properties of air, and the exhaust gas. Thus, we have to deal with two different species of gas (cf. also [KKKT03]). Let $z$ be the mass fraction of fresh gas then $1-z$ is the mass fraction of the exhaust gas. With $\sigma$ and $\tau$ defined as

$$
\sigma:=z \rho \quad \text { and } \quad \tau:=(1-z) \rho
$$


the Navier-Stokes equations (see Section 3.3 on page 31) are extended to

$$
\frac{\partial \mathbf{U}}{\partial t}+\sum_{j=1}^{3} \frac{\partial}{\partial x_{j}} \mathbf{f}_{j}(\mathbf{U})-\sum_{j=1}^{3} \frac{\partial}{\partial x_{j}} \mathbf{h}_{j}=\mathbf{0}, \quad \mathbf{x}=\left(x_{1}, x_{2}, x_{3}\right) \in \mathbb{R}^{3}, \quad t>0
$$

$$
\begin{aligned}
& \mathbf{U}=\left(\begin{array}{c}
\sigma \\
\rho \\
\rho v_{1} \\
\rho v_{2} \\
\rho v_{3} \\
e
\end{array}\right) \\
& \mathbf{f}_{1}(\mathbf{U})=\left(\begin{array}{c}
\sigma v_{1} \\
\rho v_{1} \\
\rho v_{1}^{2}+p \\
\rho v_{1} v_{2} \\
\rho v_{1} v_{3} \\
(e+p) v_{1}
\end{array}\right), \quad \mathbf{f}_{2}(\mathbf{U})=\left(\begin{array}{c}
\sigma v_{2} \\
\rho v_{2} \\
\rho v_{1} v_{2} \\
\rho v_{2}^{2}+p \\
\rho v_{2} v_{3} \\
(e+p) v_{2}
\end{array}\right), \quad \mathbf{f}_{3}(\mathbf{U})=\left(\begin{array}{c}
\sigma v_{3} \\
\rho v_{3} \\
\rho v_{1} v_{3} \\
\rho v_{2} v_{3} \\
\rho v_{3}^{2}+p \\
(e+p) v_{3}
\end{array}\right) \\
& \mathbf{h}_{j}\left(\mathbf{U}, \frac{\partial \mathbf{U}}{\partial x_{1}}, \frac{\partial \mathbf{U}}{\partial x_{2}}, \frac{\partial \mathbf{U}}{\partial x_{3}}\right)=\left(\begin{array}{c}
0 \\
0 \\
\tau_{j 1} \\
\tau_{j 2} \\
\tau_{j 3} \\
\sum_{l=1}^{3} \tau_{j l} v_{l}+\lambda \frac{\partial T}{\partial x_{j}}
\end{array}\right), \quad 1 \leq j \leq 3
\end{aligned}
$$

with

$$
\tau_{i j}=\eta\left(\frac{\partial v_{i}}{\partial x_{j}}+\frac{\partial v_{j}}{\partial x_{i}}\right)-\frac{2}{3} \eta \delta_{i j} \operatorname{div} \mathbf{v}
$$

\subsubsection{The Influence of the Air-Fuel Ratio}

As stated in Paragraph 2.6.3 on page 22, the air-fuel ratio has a considerable effect on the composition of the exhaust gas. It determines the amount of oxygen or carbon monoxide present. But the influence of this composition on heat capacities $c_{\mathrm{V}}, c_{p}$, adiabatic exponent $\gamma$, viscosity $\eta$ and thermal conductivity $\lambda$ of the mixture is less than $3 \%$ (cf. [B196]). So the effect of the $A F R$ is neglected and a stoichiometric $A F R$ of 15 is assumed. Thus, the aforementioned composition of exhaust gas (see Section 2.6.3 on page 22) is used in the calculations of the material properties. 


\subsection{Temperature Dependency of the Material Proper- ties}

As noted in Section 3.2 on page 28 the material properties are temperaturedependent: heat capacities $c_{\mathrm{V}}=c_{\mathrm{V}}(T), c_{p}=c_{p}(T)$, adiabatic exponent $\gamma=\gamma(T)$, viscosity $\eta=\eta(T)$ and thermal conductivity $\lambda=\lambda(T)$. In Figures 3.8.1 this temperature dependency is pointed out. See also test case 9.5 .2 on page 163 for a numerical evaluation of the effects of this temperature dependency during the Otto Cycle.
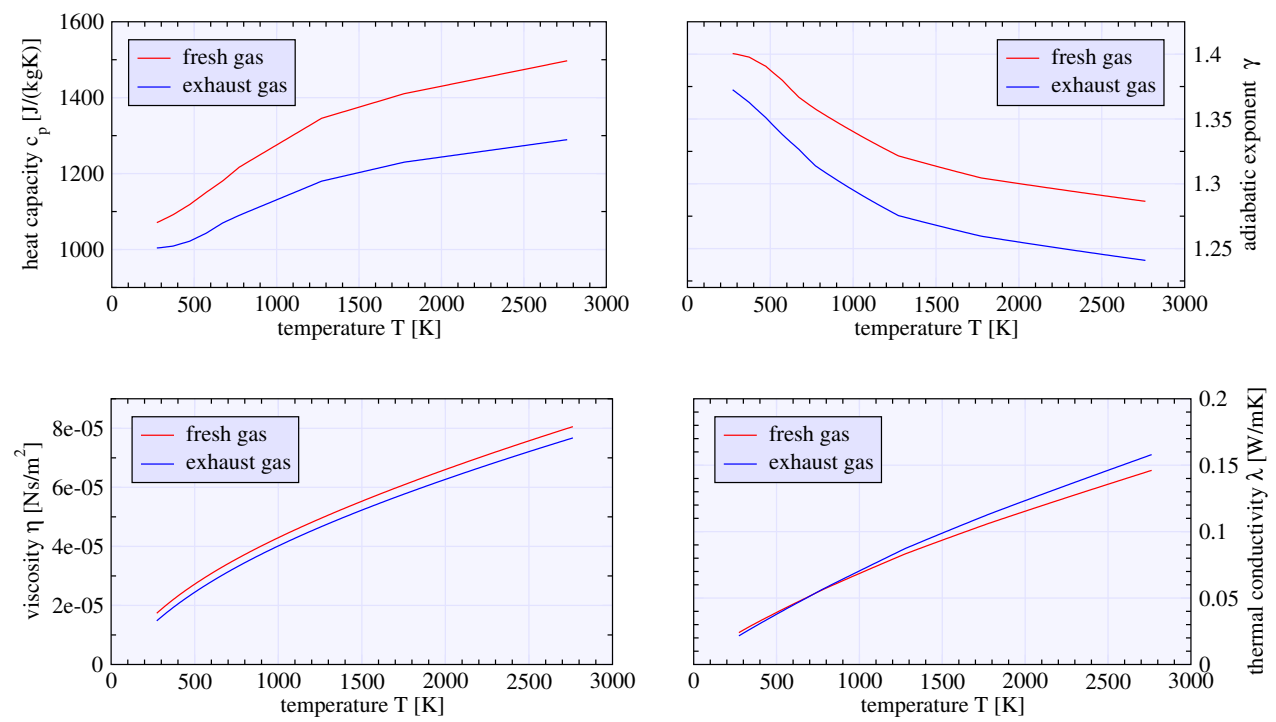

Figure 3.8.1: Temperature-dependency of heat capacity $C_{p}$ (upper left), adiabatic exponent $\gamma$ (upper right), viscosity $\eta$ (lower left), and thermal conductivity $\lambda$ (lower right).

\subsubsection{Notations}

Table 3.1: Notations for the calculation of the temperature-dependent material properties (Values for the entries that are marked by $\left({ }^{*}\right)$ can be found in Appendix B on page 209)

\begin{tabular}{|cl|}
\hline variable & description \\
\hline \hline$z_{i}$ & mass fraction of species $i$ \\
$z_{\text {air }}$ & mass fraction of air in the gas mixture \\
$z_{\text {exhaust }}$ & mass fraction of exhaust gas in the gas mixture \\
$c_{p m}$ & heat capacity at constant pressure of the mixture \\
$c_{p i}$ & heat capacity at constant pressure of species $i(*)$ \\
\hline
\end{tabular}


continued from previous page

\begin{tabular}{|cl|}
\hline variable & description \\
\hline \hline$T_{r}:=\frac{T}{T_{c}}$ & reduced temperature \\
$T_{c}$ & critical temperature $\left(^{*}\right)$ \\
$T_{c m}$ & critical temperature of the mixture \\
$T_{c i}$ & critical temperature of species $i\left(^{*}\right)$ \\
$p_{c}$ & critical pressure $\left(^{*}\right)$ \\
$p_{c m}$ & critical pressure of the mixture \\
$p_{c i}$ & critical pressure of species $i\left(^{*}\right)$ \\
$Z_{c}$ & critical compression factor $\left(^{*}\right)$ \\
$Z_{c m}$ & critical compression factor of the mixture \\
$Z_{c i}$ & critical compression factor of species $i\left(^{*}\right)$ \\
$v_{c m}$ & critical volume of the mixture \\
$v_{c m i}$ & critical volume of species $i(*)$ \\
$\mathcal{R}$ & universal gas constant $(*)$ \\
$N_{A}$ & Avogadro constant $(*)$ \\
$M^{*}$ & molar mass $(*)$ \\
$M_{m}$ & molar mass of the mixture \\
$M_{i}$ & molar mass of species $i\left(^{*}\right)$ \\
$\lambda_{m}$ & thermal conductivity of the mixture \\
$\lambda_{i}$ & thermal conductivity of species $i$ \\
$\eta_{i}$ & viscosity of species $i$ \\
\hline
\end{tabular}

\subsubsection{Formulas}

There are two possibilities of calculating the constants: one can interpolate tabulated values or evaluate an explicit formula.

For the heat capacity $\mathbf{c}_{\mathbf{p}}$ and $\mathbf{c}_{\mathrm{V}}$ it is difficult to find a formula which is accurate enough, on the other hand tabulated data is readily available (see Table B.4 on page 210 taken from [Vdi97]). So in this case interpolations of tabulated data for $c_{p}$ is used. For mixtures of ideal gases the exact mixture formula

$$
c_{p m}:=\sum_{i} z_{i} c_{p i}
$$

with mass fraction $z_{i}$ is applied. The heat capacity at constant volume $c_{\mathrm{V}}$ can be deduced with equation (3.2.7) on page 29.

The adiabatic exponent $\boldsymbol{\gamma}$ is calculated from the heat capacities via (3.2.9) on page 29 . 
For the viscosity $\boldsymbol{\eta}$ of ideal gases usually the formula of Sutherland (cf. [Tru89]) is used. A further refinement is described in [Vdi97]:

$$
\eta:=(\eta \xi)^{r} f_{p} \frac{1}{\xi}
$$

where

$$
\begin{gathered}
(\eta \xi)^{r}:=0.807 T_{r}^{0.618}-0.357 e^{-0.449 T_{r}}+0.340 e^{-4.058 T_{r}}+0.018, \\
\xi:=\frac{\left(T_{c} \mathcal{R}\right)^{\frac{1}{6}} N_{A}^{\frac{1}{3}}}{\left(M p_{c}\right)^{\frac{1}{2}}}
\end{gathered}
$$

the polarity factor $f_{p}$ for water is approximated by

$$
f_{p}:=1+30.55\left(0.292-Z_{c}\right)^{1.72}\left[0.96+0.1\left(T_{r}-0.7\right)\right]
$$

and for all other substances set to

$$
f_{p}:=1 \text {. }
$$

With this formula the values for air and the different components of the exhaust gas (see Section 3.7 on page 37) are calculated. The exhaust gas as mixture of its components is evaluated with the 1-fluid approximation where the mixture is assumed to be a new fluid with a blend of the characteristic properties of its components: the critical temperature of the mixture

$$
T_{c m}:=\sum_{i} z_{i} T_{c i}
$$

the critical pressure of the mixture

$$
p_{c m}:=\frac{\mathcal{R} Z_{c m} T_{c m}}{v_{c m}}
$$

with critical compression factor $Z_{c m}=\sum_{i} z_{i} Z_{c i}$ and critical volume $v_{c m}=\sum_{i} z_{i} v_{c i}$, the molar mass $M_{m}=\sum_{i} z_{i} M_{i}$ and the polarity factor $f_{p m}=\sum_{i} z_{i} f_{i}$.

It would be very expensive to evaluate these formulas each time the viscosity is needed. Instead a table is filled in one degree steps with the viscosity of air and exhaust gas. The resulting viscosity is then taken as a linear mixture from the table:

$$
\eta(T):=z_{\text {air }} \eta_{\text {air }}(T)+z_{\text {exhaust }} \eta_{\text {exhaust }}(T)
$$

For the thermal conductivity $\boldsymbol{\lambda}$ [Vdi97] recommends the calculation method of BROMLEY:

$$
\lambda:=\left(1.3 c_{\mathrm{V}}+1.762 \mathcal{R}-\frac{0.352 \mathcal{R}}{T_{r}}\right) \frac{\eta}{M}
$$

For the thermal conductivity $\lambda_{m}$ of a mixture of components the formula of WASSILJEWA is suggested:

$$
\lambda_{m}:=\sum_{i} \frac{z_{i} \lambda_{i}}{\sum_{j} z_{j} A_{i j}}
$$


with

$$
A_{i j}:=\frac{\left[1+\left(\frac{\eta_{i}}{\eta_{j}}\right)^{\frac{1}{2}}\left(\frac{M_{i}}{M_{j}}\right)^{\frac{1}{4}}\right]}{\left[8\left(1+\frac{M_{i}}{M_{j}}\right)\right]^{\frac{1}{2}}} .
$$

Like the viscosity the thermal conductivity of air and exhaust gas is stored in a table and blended linearly as needed:

$$
\lambda(T):=z_{\text {air }} \lambda_{\text {air }}(T)+z_{\text {exhaust }} \lambda_{\text {exhaust }}(T) .
$$




\section{Chapter 4}

\section{The Numerical Scheme}

The Euler equations (3.3.1) and Navier-Stokes equations (3.3.4) are analytically solvable for only a limited number of special cases. For more complex applications the solutions have to be approximated by the use of a discretization method. Usually, for the space discretization a mesh is fitted to the geometry and on each cell of the mesh an algebraic system of equations for the values of the unknowns at the mesh points is solved. Also, in time-dependent problems, the time interval of interest is discretized into several small time-steps.

Furthermore, the equations themselves have to be discretized. The most important methods to approach the differential equations by a system of algebraic equations are the finite difference ( $F D)$, finite element $(F E)$, and finite volume $(F V)$ methods. As FD methods are especially suited for structured meshes they are ruled out for our application, which consists of a complex geometry that cannot be handled by a structured mesh. The FV methods are known to be very well suited for the treatment of conservation laws. This is founded in the fact that they ensure the conservation property, which is the underlying principle of the system of equations (3.3.1), at the discrete level by discretizing the integral form of the conservation laws. Furthermore, they take full advantage of arbitrary meshes. Therefore, the FV approach is used here.

\section{$4.1 \quad$ Notations}

Table 4.1: Notations for the definition of the mesh structure (see also Figure 4.1.1 on the following page)

\begin{tabular}{|cl|}
\hline variable & description \\
\hline \hline $\mathcal{T}$ & unstructured mesh \\
$\Omega$ & subset of $\mathbb{R}^{3}$ \\
$\mathcal{I}$ & finite set of indices \\
$T_{j}$ & element of $\mathcal{T}$ with index $j \in \mathcal{I}$ \\
\hline
\end{tabular}

continued on next page 
continued from previous page

\begin{tabular}{|cl|}
\hline variable & description \\
\hline \hline$\left|T_{j}\right|$ & volume of element $T_{j}$ \\
$\mathbf{w}_{j}$ & center of gravity of element $T_{j}$ \\
$k_{j}$ & number of faces of element $T_{j}$ \\
$T_{j l}$ & $l^{\text {th }}$ neighbor element of element $T_{j}\left(1 \leq l \leq k_{j}\right)$ \\
$S_{j l}$ & $l^{\text {th }}$ face of element $T_{j}\left(1 \leq l \leq k_{j}\right)$ \\
$\left|S_{j l}\right|$ & area of face $S_{j l}\left(1 \leq l \leq k_{j}\right)$ \\
$\mathbf{z}_{j l}$ & center of face $S_{j l}\left(1 \leq l \leq k_{j}\right)$ \\
$\boldsymbol{\nu}_{j l}$ & outer normal to face $S_{j l}$ with length $\left|S_{j l}\right|\left(1 \leq l \leq k_{j}\right)$ \\
$\mathbf{n}_{j l}$ & outer unit normal to face $S_{j l}\left(1 \leq l \leq k_{j}\right)$ \\
\hline
\end{tabular}

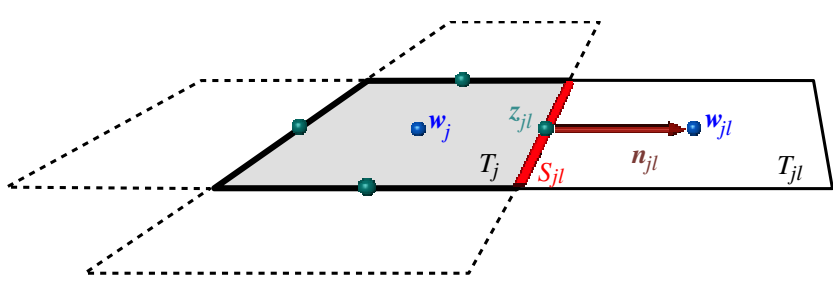

Figure 4.1.1: Mesh-related notations.

\subsection{The Mesh Structure}

Here we treat the three-dimensional case.

Remark 4.2.1. The case of curvilinear element faces with non-constant outer normals $\boldsymbol{\nu}_{j l}$ and $\mathbf{n}_{j l}$ is covered in Section 5.3 on page 78.

Definition 4.2.2. (Unstructured conformal mesh) Let $\mathcal{I} \subset \mathbb{N}$ be a finite set of indices. The set

$$
\mathcal{T}:=\left\{T_{i} \mid T_{i} \text { is a compact polyhedron for } i \in \mathcal{I}\right\},
$$

is called an unstructured conformal mesh of $\Omega \subset \mathbb{R}^{3}$, if

1. $\Omega=\bigcup_{i \in I} T_{i}$,

2. $\forall T_{i}, T_{j}(i \neq j)$ we have

- $T_{i} \cap T_{j}=\emptyset$ or 
- $T_{i} \cap T_{j}$ is a common vertex, edge, or face of $T_{i}$ and $T_{j} .{ }^{1}$

Remark 4.2.3. In a conformal mesh no hanging knots, edges, or faces are possible. With local refinement of a grid consisting of hexahedrons, such hanging faces occur (see Figure 4.2.1). Therefore, we need the following definition.

Definition 4.2.4. (Unstructured non-conformal mesh) In an unstructured nonconformal mesh constraint (2) in Definition 4.2 .2 on the preceding page reduces to

2.' $\forall T_{i}, T_{j}(i \neq j)$ we have $\stackrel{\circ}{T}_{i} \cap \stackrel{\circ}{T}_{j}=\emptyset$.

Remark 4.2.5. Numerical experiments reveal that with hexahedrons, especially boundary layers are resolved more efficiently than with tetrahedrons (see Chapter 9 on page 113 and [Sch97]). Additionally, for the Snapper algorithm (introduced in Section 5.2 on page 72), which moves the piston, hexahedrons (or prisms) are necessary. Hence, we use meshes consisting of hexahedrons.

Remark 4.2.6. The geometry in our application can be assembled by several partial unstructured non-conformal meshes. The different parts are then "glued" together by the grid merging method, described in Section 5.1 on page 65 .

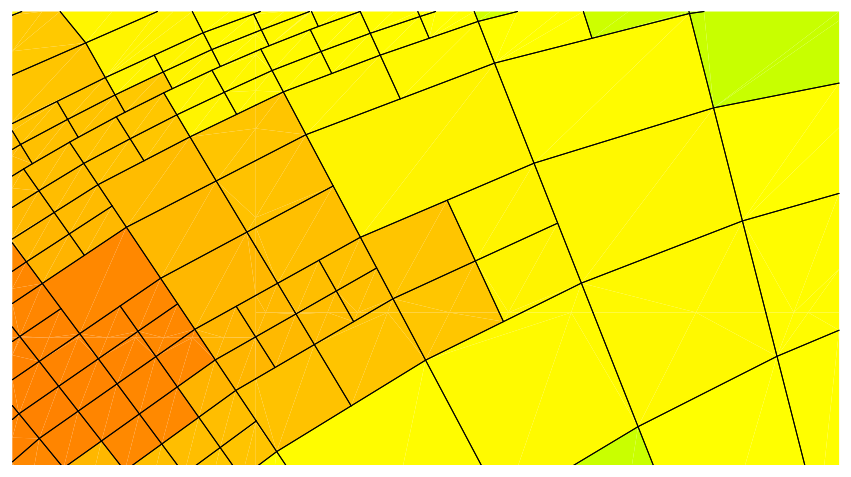

Figure 4.2.1: Hanging knots in a locally refined mesh (cross-section of a 3D mesh at the crankcase-crankshaft interface).

\subsection{The Finite Volume Scheme}

We want to derive the finite volume scheme of first order for the initial boundary value problem (3.4.2).

Remark 4.3.1. The scheme will be deduced for the Navier-Stokes equations (3.3.4). An extension to the equation system governing the gas mixture of fresh-gas and exhaust-gas (3.7.3) is straightforward.

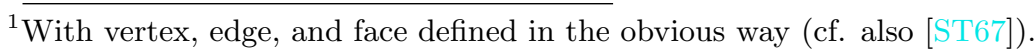


For the motivation of the finite volume scheme we assume that $\mathbf{U}$ and the functions $\mathbf{f}$ and $\mathbf{h}$ of (3.4.2) are sufficiently smooth. We start our motivation with the integral form of (3.4.2)

$$
\frac{d}{d t} \int_{T_{j}} \mathbf{U}+\int_{\partial T_{j}} \mathbf{f}(\mathbf{U}) \cdot \mathbf{n}-\int_{\partial T_{j}} \mathbf{h}(\mathbf{U}) \cdot \mathbf{n}=\mathbf{0}
$$

which one obtains from the system of Navier-Stokes equations (3.3.4) by integration over a control volume $T_{j}$ and then applying the integral theorem of Gauss.

Now, for a cell-centered ${ }^{2}$ first order scheme we define for each element $T_{j}(j \in \mathcal{I})$ and for each time $t$ the average values

$$
\mathbf{U}_{j}(t):=\frac{1}{\left|T_{j}\right|} \int_{T_{j}} \mathbf{U}(\cdot, t)
$$

and a grid function

$$
\mathbf{U}_{h}(\mathbf{x}, t):=\mathbf{U}_{j}(t) \quad \text { for } \mathbf{x} \in T_{j} .
$$

This we use to approximate equation (4.3.1) with

$$
\begin{aligned}
\frac{d}{d t} \mathbf{U}_{j}(t) & =-\frac{1}{\left|T_{j}\right|}\left(\int_{\partial T_{j}} \mathbf{f}\left(\mathbf{U}_{h}(\cdot, t)\right) \cdot \mathbf{n}-\int_{\partial T_{j}} \mathbf{h}\left(\mathbf{U}_{h}(\cdot, t)\right) \cdot \mathbf{n}\right) \\
& =-\frac{1}{\left|T_{j}\right|}\left(\sum_{l=1}^{k_{j}} \int_{S_{j l}} \mathbf{f}\left(\mathbf{U}_{h}(\cdot, t)\right) \cdot \mathbf{n}_{j l}-\sum_{l=1}^{k_{j}} \int_{S_{j l}} \mathbf{h}\left(\mathbf{U}_{h}(\cdot, t)\right) \cdot \mathbf{n}_{j l}\right) .
\end{aligned}
$$

The grid function $\mathbf{U}_{h}$ is not necessarily continuous over the cell boundaries, therefore, the flux functions $\mathbf{f}\left(\mathbf{U}_{h}\right)$ and $\mathbf{h}\left(\mathbf{U}_{h}\right)$ are not well defined on $S_{j l}$ and have to be approximated by numerical flux functions $\mathbf{g}_{j l}$ and $\mathbf{G}_{j l}$ respectively (see Paragraph 4.3 .2 on the next page, and Sections 4.4 on page 50 and 4.5 on page 52).

\subsubsection{The Time Derivative}

The time derivative is an ordinary differential equation and can be approximated by a first order explicit Euler scheme (see [Krö97]).

Let $\mathbf{U}_{j}^{n}$ be the approximation of the exact solution $\mathbf{U}(\mathbf{x}, t)$ at time level $t^{n}$ with $n \in \mathbb{N}_{0}$ such that

$$
\begin{aligned}
\mathbf{U}_{j}^{0}: & =\frac{1}{\left|T_{j}\right|} \int_{T_{j}} \mathbf{U}_{0}(\cdot), \\
\mathbf{U}_{j}^{n+1} & :=\mathbf{U}_{j}(t) \quad \text { for } t^{n}<t \leq t^{n+1} .
\end{aligned}
$$

It follows for the grid function $\mathbf{U}_{h}$ :

$$
\mathbf{U}_{h}(\mathbf{x}, t)=\mathbf{U}_{j}^{n+1} \quad \text { for } \mathbf{x} \in T_{j}, t^{n}<t \leq t^{n+1} .
$$

The explicit Euler scheme is then given by

$$
\mathbf{U}_{j}^{n+1}=\mathbf{U}_{j}^{n}-\frac{\Delta t^{n}}{\left|T_{j}\right|}\left(\sum_{l=1}^{k_{j}} \int_{S_{j l}} \mathbf{g}_{j l}\left(\mathbf{U}_{j}^{n}, \mathbf{U}_{j l}^{n}\right) \cdot \mathbf{n}_{j l}-\sum_{l=1}^{k_{j}} \int_{S_{j l}} \mathbf{G}_{j l}\left(\mathbf{U}_{j}^{n}, \mathbf{U}_{j l}^{n}\right) \cdot \mathbf{n}_{j l}\right)
$$

with $t^{n+1}=t^{n}+\Delta t^{n}$ and $\mathbf{g}_{j l}, \mathbf{G}_{j l}$ defined in the following Paragraph 4.3.2.

\footnotetext{
2 The data is located in the cell center and not on the vertices.
} 


\subsubsection{The Numerical Fluxes}

The numerical flux functions $\mathbf{g}_{j l}$ and $\mathbf{G}_{j l}$ are approximations of the integral of the flux functions $\mathbf{f}\left(\mathbf{U}_{h}\right)$ and $\mathbf{h}\left(\mathbf{U}_{h}\right)$ over the face $S_{j l}$. These fluxes are influenced by the values on both sides of the face $S_{j l}$, therefore, the numerical fluxes $\mathbf{g}_{j l}$ and $\mathbf{G}_{j l}$ depend on the approximate solution $\mathbf{U}_{j}$ and $\mathbf{U}_{j l}$ :

$$
\begin{aligned}
\mathbf{g}_{j l}\left(\mathbf{U}_{j}^{n}, \mathbf{U}_{j l}^{n}\right) & \approx\left|S_{j l}\right| \mathbf{f}\left(\mathbf{U}_{h}\left(\mathbf{z}_{j l}, t^{n}\right)\right) \cdot \mathbf{n}_{j l} \approx \int_{S_{j l}} \mathbf{f}\left(\mathbf{U}_{h}\left(\cdot, t^{n}\right)\right) \cdot \mathbf{n}_{j l} \\
\mathbf{G}_{j l}\left(\mathbf{U}_{j}^{n}, \mathbf{U}_{j l}^{n}\right) & \approx\left|S_{j l}\right| \mathbf{h}\left(\mathbf{U}_{h}\left(\mathbf{z}_{j l}, t^{n}\right)\right) \cdot \mathbf{n}_{j l} \approx \int_{S_{j l}} \mathbf{h}\left(\mathbf{U}_{h}\left(\cdot, t^{n}\right)\right) \cdot \mathbf{n}_{j l}
\end{aligned}
$$

where the integral over the face $S_{j l}$ is approximated by the value at its center $\mathbf{z}_{j l}$.

For details concerning the numerical flux functions see the following Sections 4.4 and 4.5 on page 52 .

Assumption 4.3.2. If $\mathbf{U}_{h}$ is continuous over the face $S_{j l}$ the flux functions $\mathbf{f}\left(\mathbf{U}_{h}\right)$ and $\mathbf{h}\left(\mathbf{U}_{h}\right)$ can be evaluated and the numerical fluxes should be identical to the analytical ones. Therefore, consistency is required for the numerical fluxes:

$$
\begin{aligned}
& \mathbf{g}_{j l}(\mathbf{U}, \mathbf{U})=\left|S_{j l}\right| \mathbf{f}(\mathbf{U}) \cdot \mathbf{n}_{j l} \\
& \mathbf{G}_{j l}(\mathbf{U}, \mathbf{U})=\left|S_{j l}\right| \mathbf{h}(\mathbf{U}) \cdot \mathbf{n}_{j l}
\end{aligned}
$$

In order to get the conservation property on the discrete level, the fluxes of two neighboring cells $T_{i}$ and $T_{j}$ calculated from either side of the face $S_{i j}$ need to be identical:

$$
\begin{aligned}
& \mathbf{g}_{i j}(\mathbf{U}, \mathbf{V})=-\mathbf{g}_{k l}(\mathbf{V}, \mathbf{U}) \\
& \mathbf{G}_{i j}(\mathbf{U}, \mathbf{V})=-\mathbf{G}_{k l}(\mathbf{V}, \mathbf{U})
\end{aligned}
$$

with $k$ being the index of the neighboring element, and $l$ the local number of the corresponding edge.

Furthermore, we assume that a third property, the local Lipschitz condition, is satisfied by the numerical fluxes:

$$
\begin{aligned}
\left|\mathbf{g}_{j l}(\mathbf{U}, \mathbf{V})-\mathbf{g}_{j l}\left(\mathbf{U}^{\prime}, \mathbf{V}^{\prime}\right)\right| & \leq c_{1}(R) h\left(\left|\mathbf{U}-\mathbf{U}^{\prime}\right|+\left|\mathbf{V}-\mathbf{V}^{\prime}\right|\right) \\
\left|\mathbf{G}_{j l}(\mathbf{U}, \mathbf{V})-\mathbf{G}_{j l}\left(\mathbf{U}^{\prime}, \mathbf{V}^{\prime}\right)\right| & \leq c_{2}(R) h\left(\left|\mathbf{U}-\mathbf{U}^{\prime}\right|+\left|\mathbf{V}-\mathbf{V}^{\prime}\right|\right)
\end{aligned}
$$

where $R \in \mathbb{R}^{+}, \mathbf{U}, \mathbf{V}, \mathbf{U}^{\prime}, \mathbf{V}^{\prime} \in B_{R}(0), c_{1}(R)$ and $c_{2}(R)$ are constants that depend only on $R$, and $h$ the mesh-size.

Now we are ready for the following

Definition 4.3.3. (Finite volume scheme of first order) For given initial values $\mathbf{U}_{0} \in L^{\infty}\left(\mathbb{R}^{3}\right)$ let $\mathbf{U}_{j}^{n}$ be given by:

$$
\begin{aligned}
\mathbf{U}_{j}^{0} & :=\frac{1}{\left|T_{j}\right|} \int_{T_{j}} \mathbf{U}_{0}(\cdot) \\
\mathbf{U}_{j}^{n+1} & :=\mathbf{U}_{j}^{n}-\frac{\Delta t^{n}}{\left|T_{j}\right|}\left(\sum_{l=1}^{k_{j}} \mathbf{g}_{j l}\left(\mathbf{U}_{j}^{n}, \mathbf{U}_{j l}^{n}\right)-\sum_{l=1}^{k_{j}} \mathbf{G}_{j l}\left(\mathbf{U}_{j}^{n}, \mathbf{U}_{j l}^{n}\right)\right)
\end{aligned}
$$

with the numerical flux functions satisfying properties (4.3.2), (4.3.3) and (4.3.4). 
Remark 4.3.4. If the face $S_{j l}$ is a boundary of the domain a special boundary treatment, as described in Section 4.7 on page 55 , is necessary.

Remark 4.3.5. Up to now there are no general existence results for systems in three space dimensions. Therefore, no convergence results for numerical schemes exist in this case. But if the numerical scheme in Definition 4.3.3 on the preceding page defines a convergent sequence the limit is a weak solution of the conservation law.

\subsection{The Convective Flux}

For the calculation of the convective flux function $\mathbf{g}=\mathbf{g}(\mathbf{U}, \mathbf{V})$ in three space dimensions we use a one-dimensional approach. ${ }^{3}$ The approximate solution $\mathbf{U}_{j}$ and $\mathbf{U}_{j l}$ of the elements $T_{j}$ and $T_{j l}$ respectively are rotated by an orthogonal matrix $R\left(\mathbf{n}_{j l}\right)$ such that the outer normal $\mathbf{n}_{j l}$ is transformed into the unit vector $\mathbf{e}_{1}=$ $(1,0,0)^{T}$. These rotations $R\left(\mathbf{n}_{j l}\right)$ affect only the velocity vector $\mathbf{v}$ in $\mathbf{U}_{j}$ and $\mathbf{U}_{j l}$, the scalar quantities density $\rho$ and total energy $e$ are not concerned. Now it is possible to apply a one-dimensional scheme to the rotated values

$$
\hat{\mathbf{U}}_{j}=R\left(\mathbf{n}_{j l}\right) \mathbf{U}_{j}, \quad \hat{\mathbf{U}}_{j l}=R\left(\mathbf{n}_{j l}\right) \mathbf{U}_{j l}
$$

to calculate the numerical flux function $\mathbf{g}\left(\hat{\mathbf{U}}_{j}, \hat{\mathbf{U}}_{j l}\right)$. This flux is then rotated back into its original orientation. Therefore, we have

$$
\mathbf{g}_{j l}\left(\mathbf{U}_{j}, \mathbf{U}_{j l}\right)=\left|S_{j l}\right| R^{-1}\left(\mathbf{n}_{j l}\right) \mathbf{g}\left(\hat{\mathbf{U}}_{j}, \hat{\mathbf{U}}_{j l}\right) .
$$

To calculate the numerical fluxes for the one-dimensional scheme mentioned above, we use the AUSMDV scheme proposed by Liou and Steffen in [LS93] and enhanced by WADA and LIOU in [WL97] (see also [EKS99, EKS+98]). We extended this scheme to the handling of the fresh-gas component $\sigma$ that we present now. As mentioned in Paragraph 4.3.2 on the page before, the numerical convective flux function should approximate

$$
\mathbf{g}\left(\hat{\mathbf{U}}_{j}, \hat{\mathbf{U}}_{j l}\right) \approx \mathbf{f}\left(\hat{\mathbf{U}}_{h}\right)=\left(\begin{array}{c}
\sigma u \\
\rho u \\
\rho u^{2}+p \\
(e+p) u
\end{array}\right),
$$

with $\mathbf{f}$ analogous to the function defined in Paragraph 3.7 .3 on page 38 for the rotated discrete solution $\hat{\mathbf{U}}_{h}$, and $u$ the rotated velocity vector $\mathbf{v}$ as described above. Now, the numerical flux function is defined by the AUSMDV scheme

$$
\mathbf{g}\left(\hat{\mathbf{U}}_{j}, \hat{\mathbf{U}}_{j l}\right):=\left(\begin{array}{c}
(\sigma u)_{\frac{1}{2}} \\
(\rho u)_{\frac{1}{2}} \\
\left(\rho u^{2}\right)_{\frac{1}{2}}^{A U S M D}+p_{\frac{1}{2}} \\
(\rho u)_{\frac{1}{2}}\left(\frac{e+p}{\rho}\right)_{\frac{1}{2}}
\end{array}\right)
$$

\footnotetext{
${ }^{3}$ This is possible due to the rotational invariance of the Euler and Navier-Stokes equations.
} 
with the fresh-gas flux $(\sigma u)_{\frac{1}{2}}$ and the mass flux $(\rho u)_{\frac{1}{2}}$ given by

$$
\begin{aligned}
(\sigma u)_{\frac{1}{2}} & :=u_{j}^{+} \sigma_{j}+u_{j l}^{-} \sigma_{j l}, \\
(\rho u)_{\frac{1}{2}} & :=u_{j}^{+} \rho_{j}+u_{j l}^{-} \rho_{j l},
\end{aligned}
$$

where

$$
\begin{aligned}
u_{j}^{+}:= \begin{cases}\alpha_{j} \frac{\left(u_{j}+c_{m}\right)^{2}}{4 c_{m}}+\left(1-\alpha_{j}\right) \frac{u_{j}+\left|u_{j}\right|}{2} & \text { if }\left|u_{j}\right| \leq c_{m} \\
\frac{u_{j}+\left|u_{j}\right|}{2} & \text { otherwise }\end{cases} \\
u_{j l}^{-}:= \begin{cases}-\alpha_{j l} \frac{\left(u_{j l}-c_{m}\right)^{2}}{4 c_{m}}+\left(1-\alpha_{j l}\right) \frac{u_{j l}-\left|u_{j l}\right|}{2} & \text { if }\left|u_{j l}\right| \leq c_{m} \\
\frac{u_{j l}+\left|u_{j l}\right|}{2} & \text { otherwise },\end{cases} \\
\alpha_{j}:=\frac{2 p_{j} / \rho_{j}}{p_{j} / \rho_{j}+p_{j l} / \rho_{j l}}, \quad \alpha_{j l}:=\frac{2 p_{j l} / \rho_{j l}}{p_{j} / \rho_{j}+p_{j l} / \rho_{j l}}, \\
c_{m}:=\max \left(c_{j}, c_{j l}\right) .
\end{aligned}
$$

$\left(\rho u^{2}\right)_{\frac{1}{2}}^{A U S M D V}$ is defined as

$$
\left(\rho u^{2}\right)_{\frac{1}{2}}^{A U S M D V}:=\frac{1}{2}(1+s)\left(\rho u^{2}\right)_{\frac{1}{2}}^{A U S M V}+\frac{1}{2}(1-s)\left(\rho u^{2}\right)_{\frac{1}{2}}^{A U S M D}
$$

with

$$
\begin{aligned}
\left(\rho u^{2}\right)_{\frac{1}{2}}^{A U S M V} & :=u_{j}^{+} \rho_{j} u_{j}+u_{j l}^{-} \rho_{j l} u_{j l}, \\
\left(\rho u^{2}\right)_{\frac{1}{2}}^{A U S M D} & :=\left\{\begin{array}{cc}
(\rho u)_{\frac{1}{2}} u_{j} & \text { if }(\rho u)_{\frac{1}{2}}>0, \\
(\rho u)_{\frac{1}{2}} u_{j l} & \text { otherwise, }
\end{array}\right. \\
s & :=\min \left\{1, K \frac{\left|p_{j}-p_{j l}\right|}{\min \left\{p_{j}, p_{j l}\right\}}\right\}, \quad K:=10 .
\end{aligned}
$$

For the pressure flux $p_{\frac{1}{2}}$ we have

$$
\begin{gathered}
p_{\frac{1}{2}}:=p_{j}^{+}+p_{j l}^{-}, \\
p_{j}^{+}:= \begin{cases}p_{j} \frac{\left(u_{j}+c_{m}\right)^{2}}{4 c_{m}^{2}}\left(2-\frac{u_{j}}{c_{m}}\right) & \text { if }\left|u_{j}\right| \leq c_{m}, \\
p_{j} \frac{u_{j}+u_{j} \mid}{2 u_{j}} & \text { otherwise, }\end{cases} \\
p_{j l}^{-}:= \begin{cases}p_{j l} \frac{\left(u_{j l}-c_{m}\right)^{2}}{4 c_{m}^{2}}\left(2+\frac{u_{j l}}{c_{m}}\right) & \text { if }\left|u_{j l}\right| \leq c_{m}, \\
p_{j l} \frac{u_{j l}+\left|u_{j l}\right|}{2 u_{j l}} & \text { otherwise. }\end{cases}
\end{gathered}
$$

And finally $\left(\frac{e+p}{\rho}\right)_{\frac{1}{2}}$ is defined by

$$
\left(\frac{e+p}{\rho}\right)_{\frac{1}{2}}:= \begin{cases}\frac{e_{j}+p_{j}}{\rho_{j}} & \text { if }(\rho u)_{\frac{1}{2}}>0 \\ \frac{e_{j l}+p_{j l}}{\rho_{j l}} & \text { otherwise }\end{cases}
$$


This AUSMDV flux has the above required properties (4.3.2), (4.3.3) and (4.3.4), as can be seen by a direct calculation. It is a hybrid flux vector- flux differencesplitting scheme and has good shock capturing capabilities with little numerical viscosity. Therefore, it is especially adapted for the treatment of the convective flux in a simulation of the Navier-Stokes equations. For details of the scheme and numerous numerical experiments confer [WL97].

\subsection{The Viscous Flux}

In order to be able to calculate the viscous flux $\mathbf{G}=\mathbf{G}(\mathbf{U}, \mathbf{V})$ we need an approximation of the derivatives of $\mathbf{U}_{h}$ at the face $S_{j l}$. Therefore, a gradient has to be extracted from the element-wise constant $\mathbf{U}_{h}$. This is done with a central discretization since shear stress and heat conduction correspond to diffusive effects. The advantage of a central discretization is furthermore that it meets property (4.3.3) and therefore is conservative.

An easy approach to calculate the discrete gradient of the numerical solution between elements $T_{j}$ and $T_{j l}$ would be to use ( $u$ being the quantity in question, either the temperature $T$ or a component of the velocity $\mathbf{v}$ )

$$
\frac{\partial u}{\partial x_{i}}:=\Delta x_{i} \frac{\Delta u}{|\Delta \mathbf{x}|^{2}}
$$

with the numerical approximation of the gradient $\Delta u:=(u)_{j}-(u)_{j l}$ and, analogously $\Delta x_{i}$ and $\Delta \mathbf{x}$, as depicted on the left-hand side of Figure 4.5.1 on page 54 (cf. also [Jen97]).

This would yield a good approximation for an orthogonal mesh, i.e $\mathbf{w}_{j}-\mathbf{w}_{j l}=$ $c \mathbf{n}_{j l}, c \in \mathbb{R}$. However, in unstructured meshes for complex geometries this condition is usually violated. Therefore, it is better (cf. the simulation of the flow over a flat plate in Paragraph 9.3.3 on page 135, and [KKS01, Sch01]) to calculate the gradient by using a hyperplane that satisfies

$$
\frac{\partial u}{\partial \mathbf{t}_{j l}}=0
$$

with the tangential vector $\mathbf{t}_{j l} \cdot \mathbf{n}_{j l}=0$ (see Figure 4.5.1 (right)).

In order to construct this hyperplane we take four linearly independent points $\mathbf{P}_{i m} \in \mathbb{R}^{4}$ as supporting points. Let Points $\tilde{\mathbf{P}}_{11}=\left(x_{11}, y_{11}, z_{11}\right)$ and $\tilde{\mathbf{P}}_{21}=$ $\left(x_{21}, y_{21}, z_{21}\right)$ be the centers of gravity of $T_{j}$ and $T_{j l}$ and two further points $\tilde{\mathbf{P}}_{i 1}=$ $\left(x_{i 1}, y_{i 1}, z_{i 1}\right), i \in\{1,2\}$ satisfying $\left(\tilde{\mathbf{P}}_{i 1}-\tilde{\mathbf{P}}_{i 2}\right) \cdot \mathbf{n}_{j l}=0$, such that these four points do not belong to a plane $E \in \mathbb{R}^{3}$ (cf. Figure 4.5.1 (bottom)). Let furthermore $\mathbf{p}_{i m}=\left(x_{i m}, y_{i m}, z_{i m}, u_{i}\right), i, m \in\{1,2\}$ with $u_{1}=(u)_{j}$ and $u_{2}=(u)_{j l}$. Then, the gradient $\mathbf{D}_{j l}(u)$ of the so defined hyperplane is given by

$$
\mathbf{D}_{j l}(u)=\frac{1}{\operatorname{det} K_{j l}}\left(\begin{array}{l}
d_{1} \\
d_{2} \\
d_{3}
\end{array}\right)
$$


with

$$
\begin{gathered}
K_{j l}=\left(\begin{array}{llll}
x_{12} & y_{12} & z_{12} & 1 \\
x_{11} & y_{11} & z_{11} & 1 \\
x_{22} & y_{22} & z_{22} & 1 \\
x_{21} & y_{21} & z_{21} & 1
\end{array}\right), \\
d_{1}=\operatorname{det}\left(\begin{array}{llll}
(u)_{j} & y_{12} & z_{12} & 1 \\
(u)_{j} & y_{11} & z_{11} & 1 \\
(u)_{j l} & y_{22} & z_{22} & 1 \\
(u)_{j l} & y_{21} & z_{21} & 1
\end{array}\right), \\
d_{2}=\operatorname{det}\left(\begin{array}{llll}
x_{12} & (u)_{j} & z_{12} & 1 \\
x_{11} & (u)_{j} & z_{11} & 1 \\
x_{22} & (u)_{j l} & z_{22} & 1 \\
x_{21} & (u)_{j l} & z_{21} & 1
\end{array}\right), \\
d_{3}=\operatorname{det}\left(\begin{array}{llll}
x_{12} & y_{12} & (u)_{j} & 1 \\
x_{11} & y_{11} & (u)_{j} & 1 \\
x_{22} & y_{22} & (u)_{j l} & 1 \\
x_{21} & y_{21} & (u)_{j l} & 1
\end{array}\right),
\end{gathered}
$$

as can be verified by direct calculation.

With this gradient $\mathbf{D}_{j l}$ the numerical viscous flux $\mathbf{G}_{j l}$ can be constructed as an approximation of the analytical viscous flux defined in (3.3.4) on page 32:

$$
\mathbf{G}_{j l}\left(\mathbf{U}_{j}, \mathbf{U}_{j l}\right):=\left|S_{j l}\right| \sum_{k=1}^{3} \mathbf{H}_{j l}^{k}\left(\mathbf{U}_{j}, \mathbf{U}_{j l}\right) \cdot \mathbf{n}_{j l} \approx \int_{S_{j l}} \mathbf{h}\left(\mathbf{U}_{h}\left(\cdot, t^{n}\right)\right) \cdot \mathbf{n}_{j l}
$$

with

$$
\begin{gathered}
\mathbf{H}_{j l}^{k}\left(\mathbf{U}_{j}, \mathbf{U}_{j l}\right):=\left(\begin{array}{c}
0 \\
\tau_{k 1} \\
\tau_{k 2} \\
\tau_{k 3} \\
\sum_{l=1}^{3} \tau_{k l} \bar{v}_{l}+\lambda \mathbf{D}_{j l}^{k}(T)
\end{array}\right) \\
\tau_{r s}:=\eta\left(\mathbf{D}_{j l}^{r}\left(v_{s}\right)+\mathbf{D}_{j l}^{s}\left(v_{r}\right)\right)-\frac{2}{3} \eta \delta_{r s} \sum_{i=1}^{3} \mathbf{D}_{j l}^{i}\left(v_{i}\right), \quad r, s \in\{1,2,3\} \\
\mathbf{D}_{j l}^{k}(T) \quad \text { gradient of temperature } T \text { over face } S_{j l} \text { in direction } x_{k}, \\
\mathbf{D}_{j l}^{k}\left(v_{i}\right) \text { gradient of velocity component } v_{i} \text { over face } S_{j l} \text { in direction } x_{k}, \\
\bar{v}_{i}:=\frac{1}{2}\left(\left(v_{i}\right)_{j}+\left(v_{i}\right)_{j l}\right) .
\end{gathered}
$$

Remark 4.5.1. Comparisons with a reconstruction technique, similar to the one used in the MUSCL approach for higher order schemes (cf. [Krö97]) to obtain a linear solution function $\mathbf{U}_{j}$ on each element as described in [Sch97, WKM+98, WK96], did not show improved results. To compute the gradient with this reconstruction technique is computationally more complex, thus we use the above described, more efficient, approach (4.5.1). 

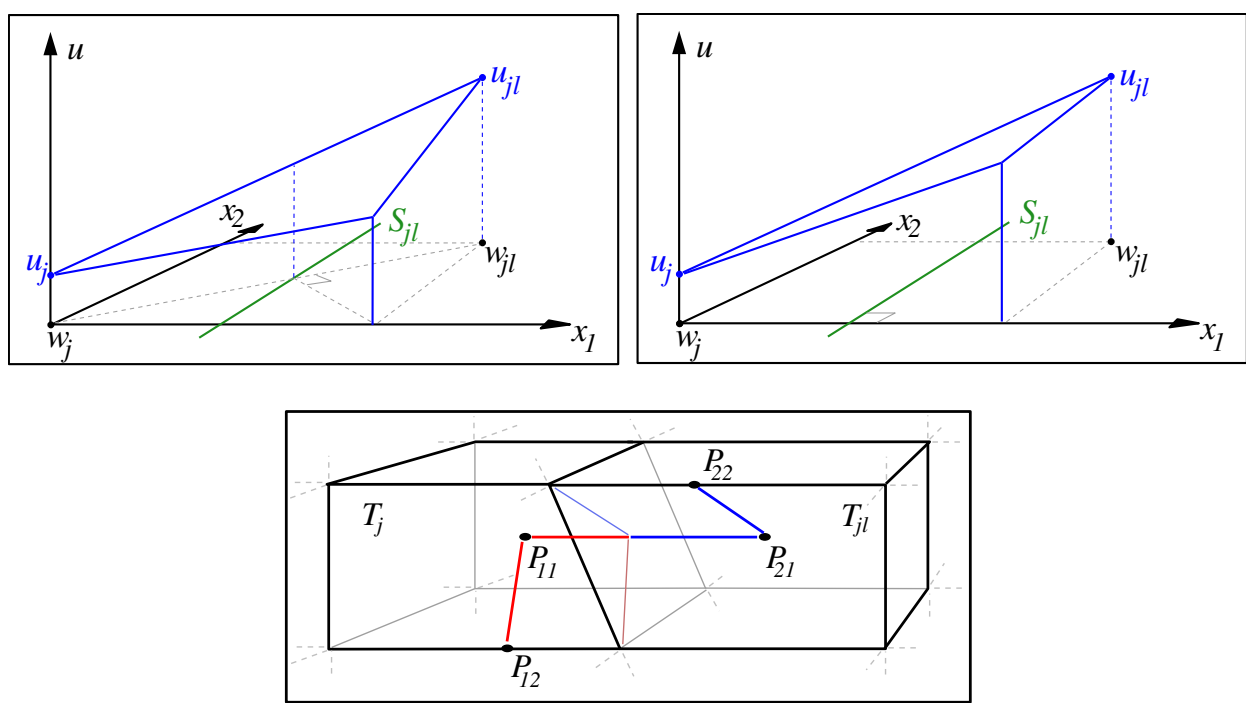

Figure 4.5.1: Gradient calculation with the naive approach (left), with the parallel hyperplane approach (middle), and supporting points for this hyperplane (right).

\subsection{The Time-Dependent Integral Form of the Navier- Stokes Equations}

As in Section 4.3 on page 47 we now want to derive equations for the numerical flux in time-dependent geometries. For this we use the identity (as in [Wie95])

$$
\frac{d}{d t} \int_{V(t)} \phi(t, \mathbf{x}) d \mathbf{x}=\int_{V(t)} \frac{\partial}{\partial t} \phi(t, \mathbf{x}) d \mathbf{x}+\int_{\partial V(t)} \phi(t, \mathbf{x}) \mathbf{w}(t, \mathbf{x}) \cdot \mathbf{n}
$$

with an arbitrary time-dependent volume $V(t)$, a scalar function $\phi(t, \mathbf{x}), \mathbf{w}(t, \mathbf{x})$ the velocity of $\partial V(t)$, and $\mathbf{n}$ the outer normal of $\partial V(t)$.

As in the motivation of the numerical scheme (cf. Section 4.3 on page 47), we use the integral form of the system of Navier-Stokes equations (3.3.4), with the numerical flux functions $\mathbf{f}_{1}, \mathbf{f}_{2}$, and $\mathbf{f}_{3}$ as defined in (3.3.3), that is obtained by integration over a control volume $T_{j}$ and then by applying the integral theorem of Gauss. But in the case of time-dependent geometries, the additional boundary term $\int_{\partial V(t)} \phi(t, \mathbf{x}) \mathbf{w}(t, \mathbf{x}) \cdot \mathbf{n}$ from equation (4.6.1) has to be taken into account. This boundary term can be combined with the numerical flux functions $\mathbf{f}_{1}, \mathbf{f}_{2}$, and $\mathbf{f}_{3}$, resulting in the new flux functions $\tilde{\mathbf{f}}_{1}, \tilde{\mathbf{f}}_{2}$, and $\tilde{\mathbf{f}}_{3}$. Thus, we have the integral form of the conservation laws for a time-dependent domain $V(t)$ :

$$
\frac{d}{d t} \int_{T_{j}} \mathbf{U}+\int_{\partial T_{j}} \tilde{\mathbf{f}}(\mathbf{U}) \cdot \mathbf{n}-\int_{\partial T_{j}} \mathbf{h}(\mathbf{U}) \cdot \mathbf{n}=\mathbf{0}
$$


with

$$
\begin{aligned}
& \tilde{\mathbf{f}}_{1}(\mathbf{U})=\left(\begin{array}{c}
\rho\left(v_{1}-w_{1}\right) \\
\rho\left(v_{1}^{2}-w_{1}\right)+p \\
\rho\left(v_{1} v_{2}-w_{1}\right) \\
\rho\left(v_{1} v_{3}-w_{1}\right) \\
(e+p)\left(v_{1}-w_{1}\right)
\end{array}\right), \\
& \tilde{\mathbf{f}}_{2}(\mathbf{U})=\left(\begin{array}{c}
\rho\left(v_{2}-w_{2}\right) \\
\rho\left(v_{1} v_{2}-w_{2}\right) \\
\rho\left(v_{2}^{2}-w_{2}\right)+p \\
\rho\left(v_{2} v_{3}-w_{2}\right) \\
(e+p)\left(v_{2}-w_{2}\right)
\end{array}\right) \text {, } \\
& \tilde{\mathbf{f}}_{3}(\mathbf{U})=\left(\begin{array}{c}
\rho\left(v_{3}-w_{3}\right) \\
\rho\left(v_{1} v_{3}-w_{3}\right) \\
\rho\left(v_{2} v_{3}-w_{3}\right) \\
\rho\left(v_{3}^{2}-w_{3}\right)+p \\
(e+p)\left(v_{3}-w_{3}\right)
\end{array}\right),
\end{aligned}
$$

and $\mathbf{h}$ as defined in (3.3.4).

\subsection{The Boundary Conditions}

For the calculation of the approximate solution $\mathbf{U}_{j}^{n+1}$ of the next time step in element $T_{j}$ the finite volume scheme relies on the numerical fluxes. These are evaluated on the basis of the values of $\mathbf{U}_{j}^{n}$ and $\mathbf{U}_{j l}^{n}$ on the neighboring elements. If the element $T_{j}$ is situated on a boundary of the computational domain $\Omega$ there are faces $S_{j l}$ where $T_{j}$ has no neighbor. In this case we use the method of ghost cells. A ghost cell $\check{T}_{j l}$ of $T_{j}$ is a mirror image of $T_{j}$ over the face $S_{j l}$. Its values $\check{\mathbf{U}}_{j l}^{n}$ depend on the type of boundary and are either specified by a physical boundary condition or obtained by extrapolation from the element $T_{j}$ itself. This ghost cell $\check{T}_{j l}$ can then be used to calculate the numerical flux over the face $S_{j l}$ with the interior finite volume scheme as described above. Therefore, this method closes the system of discrete equations on the calculation domain. It also enables the influence of the boundary condition on the flow by the upwind flux of the numerical flux function.

As seen in Section 3.4 on page 32 there are actual boundary conditions like fixed or moving walls, and artificial boundary conditions, like inflow or outflow conditions, where the physical domain is unbounded and which has to be truncated in order to get a finite domain. In both types of boundaries Dirichlet (e.g. $p=p_{0}$, prescribed pressure) and Von Neumann boundary conditions (e.g. $\frac{\partial T}{\partial n}=0$, no heat flux) (or a mixture of these) can be prescribed:

- The numerical realization of Dirichlet boundary conditions is accomplished by an extrapolation of the boundary data into the ghost cell as shown on the left-hand side of Figure 4.7.1 on the following page. For example, the pressure $\check{p}$ on the ghost cell $\check{T}_{j l}$ is calculated as

$$
\check{p}=2 p_{0}-(p)_{j}
$$


with a prescribed pressure $p_{0}$ and the pressure $(p)_{j}$ from within $T_{j}$.

- Von Neumann boundary conditions are implemented as an extrapolation of the data from the element $T_{j}$ (see Figure 4.7.1 (right)). In the above example of $\frac{\partial T}{\partial n}=0$ the temperature $\breve{T}$ of the ghost cell would be given by

$$
\check{T}=(T)_{j}
$$

with the temperature $(T)_{j}$ of element $T_{j}$.
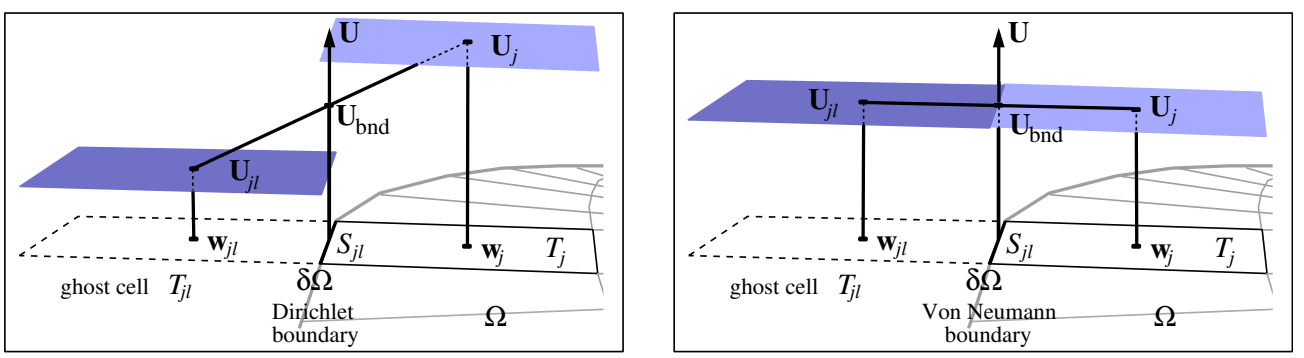

Figure 4.7.1: Dirichlet (left) and Von Neumann (right) boundary treatment.

The treatment of the boundary conditions is normally not a trivial task. As stated in Paragraph 3.4.2 on page 33, it is usually not possible to specify conditions for all variables. Depending on the type of boundary (e.g. subsonic inflow, subsonic outflow, no-slip wall condition) only a certain number of physical boundary conditions can be given. The remaining variables have to be supported by numerical boundary conditions:

- For numerical boundary conditions for a first order scheme the values are extrapolated constantly from within the domain.

With these facts in mind we can now describe the implemented boundary conditions in detail:

\subsubsection{Inflow}

As inflow boundary condition we use a mass-flow condition. Thus, density $\rho_{0}$ and velocity $\mathbf{v}_{0}$ are prescribed at this boundary. As we are in the case of a subsonic flow at the inlet the last variable has to be taken from inside the domain. The temperature $T$ is therefore treated as a numerical boundary condition. With these quantities given, $p$ can then be calculated via the thermal equation of state and the total energy $e$ by the caloric equation of state:

$$
\check{\mathbf{U}}_{j l}=\left(\begin{array}{c}
\rho_{0} \\
\rho_{0} \mathbf{v}_{0} \\
e\left((T)_{j}\right)
\end{array}\right)
$$




\subsubsection{Outflow}

At the outflow boundary the pressure $p_{0}$ is imposed. Density $\rho$ and velocity $\mathbf{v}$ are taken from the inside:

$$
\check{\mathbf{U}}_{j l}=\left(\begin{array}{c}
(\rho)_{j} \\
(\rho \mathbf{v})_{j} \\
e\left(T\left((\rho)_{j}, p_{0}\right)\right)
\end{array}\right)
$$

\subsubsection{Slip (fixed)}

As this is a solid wall condition, no mass flux should be present and the normal components of the velocity have to vanish on the boundary. Depending on the thermal properties of the wall (adiabatic or isothermal) the condition for the temperature $T$ is either a Von Neumann or a Dirichlet condition:

$$
\begin{array}{cc}
\text { adiabatic: } & \check{\mathbf{U}}_{j l}=\left(\begin{array}{c}
(\rho)_{j} \\
(\rho)_{j}\left((\mathbf{v})_{j}-2\left(\left(\mathbf{v}_{j}\right) \cdot \mathbf{n}_{j l}\right) \mathbf{n}_{j l}\right) \\
e\left((T)_{j}\right)
\end{array}\right) \\
\text { isothermal: } & \check{\mathbf{U}}_{j l}=\left(\begin{array}{c}
(\rho)_{j} \\
(\rho)_{j}\left((\mathbf{v})_{j}-2\left(\left(\mathbf{v}_{j}\right) \cdot \mathbf{n}_{j l}\right) \mathbf{n}_{j l}\right) \\
e\left(T_{0}\right)
\end{array}\right)
\end{array}
$$

This boundary condition is used for the numerical experiments in Chapter 10 on page 167.

\subsubsection{No-Slip (fixed)}

The difference to the slip boundary condition is that here also the tangential component of the velocity has to vanish on the boundary:

$$
\begin{array}{cc}
\text { adiabatic: } & \check{\mathbf{U}}_{j l}=\left(\begin{array}{c}
(\rho)_{j} \\
-(\rho \mathbf{v})_{j} \\
e\left((T)_{j}\right)
\end{array}\right) \\
\text { isothermal: } & \check{\mathbf{U}}_{j l}=\left(\begin{array}{c}
(\rho)_{j} \\
-(\rho \mathbf{v})_{j} \\
e\left(T_{0}\right)
\end{array}\right)
\end{array}
$$

\subsubsection{No-Slip (moving)}

The Moving Wall Boundary Condition can be deduced from the descriptions given in Section 4.6 on page 54. As the velocity of the fluid at a no-slip boundary is given by $\mathbf{v}=\mathbf{v}_{\text {wall }}$ the equation (4.6.1) for the convective flux over face $S_{j l}$ of element $T_{j}$ results in

$$
\int_{S_{j l}} \check{f}\left(\mathbf{U}_{j}\right) \mathbf{n}_{j l}=-\left|S_{j l}\right|\left(\begin{array}{c}
0 \\
(p)_{j}\left(\mathbf{n}_{j l}\right)_{1} \\
(p)_{j}\left(\mathbf{n}_{j l}\right)_{2} \\
(p)_{j}\left(\mathbf{n}_{j l}\right)_{3} \\
(p)_{j} \mathbf{n}_{j l} \cdot \mathbf{v}_{\text {wall }}
\end{array}\right)=: \mathbf{g}_{j l}\left(\mathbf{U}_{j}, \mathbf{U}_{j l}\right)
$$


with $\left(\mathbf{n}_{j l}\right)_{i}$ the $i^{\text {th }}$ component of the outer normal of face $S_{j l}$ and $\check{f}$ defined in (4.6.1) (cf. [DP90, Wie95, WKM+98, WK96]).

For the viscous flux the ghost cell approach, similar to the fixed no-slip condition, is used:

$$
\begin{gathered}
\text { adiabatic: } \quad \check{\mathbf{U}}_{j l}=\left(\begin{array}{c}
(\rho)_{j} \\
(\rho)_{j}\left(2 \mathbf{v}_{\text {wall }}-(\mathbf{v})_{j}\right) \\
e\left((T)_{j}\right)
\end{array}\right) \\
\text { isothermal: } \quad \check{\mathbf{U}}_{j l}=\left(\begin{array}{c}
(\rho)_{j} \\
(\rho)_{j}\left(2 \mathbf{v}_{\text {wall }}-(\mathbf{v})_{j}\right) \\
e\left(T_{0}\right)
\end{array}\right)
\end{gathered}
$$

Remark 4.7.1. The choice of the prescription of boundary conditions is by no means trivial, and one has to be careful not to get an ill-posed problem. Especially the interaction of inflow and outflow boundary has to be kept in mind. In the special case of subsonic inflow and outflow conditions one can impose two out of three independent variables at the inflow and, at the outflow boundary the remaining third one should be given (cf. [Hir92b], [PL92]).

\subsection{The CFL-Condition}

For the discretization of the time derivative we use an explicit first order Euler scheme.

Remark 4.8.1. One of the reasons why we apply an explicit scheme, is that we need a high temporal resolution for our time-dependent problem. Thus, an implicit scheme which allows us to use big time-steps would not be advantageous. Additionally, it has been shown (see e.g. [Geß31, Krö97]) that for time exact simulations an explicit time discretization is more efficient than an implicit one. The reason for this is that the error accumulates at large time-steps with the implicit scheme and hence, the mesh size has to be smaller in order to attain the same numerical error as with an explicit scheme.

However, stability and convergence conditions impose restrictions on the maximum admissible time-step for an explicit scheme. This can be motivated as follows (cf. [Hir92a, Krö97]).

\subsubsection{The Convective Part}

Remark 4.8.2. An explicit finite difference scheme for a scalar conservation law in one space dimension is stable if the time-step $\Delta t^{n}$ is restricted by the, so-called, Courant-Friedrichs-Levi condition (or CFL condition) (cf. e.g. [LeV90]):

$$
\Delta t^{n}<\mathrm{CFL} \frac{h}{\max _{x \in \Omega} f^{\prime}\left(u^{n}(x)\right)}
$$

with minimum mesh size $h$ and a constant CFL $<1$. 
Similarly, an explicit finite volume scheme for a scalar conservation law on an unstructured triangulation $\mathcal{T}$ is monotone if

$$
\Delta t^{n}<\mathrm{CFL} \min _{T_{j} \in \mathcal{T}}\left\{\frac{\left|T_{j}\right|}{\sum_{l=1}^{3} \max \left\{\nu_{j l} f^{\prime}\left(u_{j}^{n}\right), 0\right\}}\right\},
$$

with a constant CFL $<1$ (see [GKR93]).

Analogously, we define the time-step restriction for the convective part of our finite volume scheme (cf. [Geß94, Geß01, Wie95]).

Definition 4.8.3. (Finite volume time-step restriction for the convective part) For a given constant $\mathrm{CFL}<1$ let

$$
\begin{aligned}
\Delta t_{\text {conv }}^{n} & :=\operatorname{CFL} \min _{T_{j} \in \mathcal{T}}\left\{\Delta t_{j, \text { conv }}^{n}\right\} \\
\Delta t_{j, \text { conv }}^{n} & :=\frac{\left|T_{j}\right|}{\max _{l=1, \ldots, k_{j}}\left\{\left|S_{j l}\right|\left|\lambda_{j l}\left(\mathbf{U}_{j}^{n}\right)\right|\right\}},
\end{aligned}
$$

with $\lambda_{j l}\left(l=1, \ldots, k_{j}\right)$ defined (motivated by the eigenvalues of the system of Euler equations (3.3.1) (see [Krö97])) by

$$
\lambda_{j l}\left(\mathbf{U}_{j}^{n}\right):=\left|\mathbf{v}_{j}^{n} \cdot \mathbf{n}_{j l}\right|+c_{j}^{n}
$$

where $\mathbf{v}_{j}^{n}$ is the velocity of element $T_{j}$ at time-step $t^{n}$ and $c_{j}^{n}$ its local speed of sound (see 3.2 .3 on page 30 ).

Remark 4.8.4. In other words, the time-step has to be small enough so that information can not travel further than one cell during this time-step.

\subsubsection{The Viscous Part}

As the flow in our two-stroke simulation is strongly convection dominant, the viscous part of the finite volume scheme is mainly dominated by the convective one. Thus, a CFL-like condition for the viscous discretization is not as relevant. We base our choice on the partly empirical results obtained in [MJ89] for dual meshes in two dimensions. Applied to our situation, we get

$$
\begin{aligned}
\Delta t_{\text {visc }}^{n} & :=\operatorname{CFL} \min _{T_{j} \in \mathcal{T}}\left\{\Delta t_{j, \mathrm{visc}}^{n}\right\} \\
\Delta t_{j, \mathrm{visc}}^{n} & :=\chi \frac{\left|T_{j}\right|^{2} \rho_{j}}{\alpha_{\text {part }} \eta \sum_{l=1}^{k_{j}}\left|S_{j l}\right|} \text { with } \\
\alpha_{\mathrm{part}} & :=\frac{\gamma_{\text {part }}^{\frac{3}{2}} \kappa_{\text {part }}}{c_{\text {part }} \rho_{\text {part }} L_{\text {part }} C_{p, \text { part }}}
\end{aligned}
$$

where $(\cdot)_{\text {part }}$ is the average of the respective value in the part of the geometry (inlet with crankcase and transfer ports, cylinder, outlet with silencer) where element $T_{j}$ is situated, $L_{\text {part }}$ is a characteristic length scale of this part of the geometry, and $\chi=\frac{1}{4}$ specifies the relative weight of the viscous to the convective part of the time-step $\Delta t^{n}$. 


\subsubsection{The Overall Time-Step}

The time-step $\Delta t^{n}$ is composed of the above-defined convective and viscous parts (see [Sch97, WK96]):

$$
\Delta t^{n}:=\mathrm{CFL} \min _{T_{j} \in \mathcal{T}}\left\{\frac{\Delta t_{j, \mathrm{conv}}^{n} \Delta t_{j, \mathrm{visc}}^{n}}{\Delta t_{j, \mathrm{conv}}^{n}+\Delta t_{j, \mathrm{visc}}^{n}}\right\}
$$

with $\Delta t_{j, \text { conv }}^{n}$ and $\Delta t_{j \text {,visc }}^{n}$ as defined in Paragraph 4.8 .1 on page 58 and 4.8.2 on the preceding page respectively.

Remark 4.8.5. For a more efficient, local handling of this time-step restriction see Section 6.1 on page 87 .

\subsection{The General Equation of State}

In the case of a (thermally and calorically, i.e. polytropic) perfect gas the Euler equations, and therefore the convective part of the Navier-Stokes equations, can be discretized with the AUSMDV Riemann solver, which was developed for this specific problem. In the case of a calorically imperfect gas (that results from taking into account the temperature-dependent material constants (cf. Section 3.8 on page 40)), the caloric equation of state is given by equation (3.5.2) instead of (3.5.4). Thus, the prerequisites for the usage of the AUSMDV solver are not valid any more. This problem can be handled by the energy relaxation scheme first described in [CP98]. The idea of this scheme is based on the splitting of the internal energy $\varepsilon=\varepsilon_{1}+\varepsilon_{2}$ in a part $\varepsilon_{1}$ that defines the polytropic equation of state $p_{1}=(\gamma-1) \rho \varepsilon_{1}$ for a polytropic gas analogous to (3.5.4), and a part $\varepsilon_{2}$, which contains the disturbing nonlinearities of the caloric equation of state of the imperfect gas. The flux determined by the polytropic part of the internal energy $\varepsilon_{1}$ can be handled by the standard Riemann solver (AUSMDV). The second part $\varepsilon_{2}$ is only advected in this first step. In a second step, the relaxation step, the influence of the nonlinearities in $\varepsilon_{2}$ are taken into account.

\subsubsection{The Relaxation System}

The following relaxation system for $\lambda \in \mathbb{R}^{+}$is studied

$$
\left\{\begin{aligned}
\frac{\partial}{\partial t} \rho^{\lambda}+\nabla \cdot\left(\rho^{\lambda} \mathbf{v}^{\lambda}\right) & =0, \\
\frac{\partial}{\partial t}\left(\rho^{\lambda} \mathbf{v}^{\lambda}\right)+\nabla \cdot\left(\rho^{\lambda} \mathbf{v}^{\lambda}\left(\mathbf{v}^{\lambda}\right)^{T}+p_{1}^{\lambda} I\right) & =0, \\
\frac{\partial}{\partial t} e_{1}^{\lambda}+\nabla \cdot\left(\left(e_{1}^{\lambda}+p_{1}^{\lambda}\right) \mathbf{v}^{\lambda}\right) & =\lambda \rho^{\lambda}\left(\varepsilon_{2}^{\lambda}-\Phi\left(\rho^{\lambda}, \varepsilon_{1}^{\lambda}\right)\right), \\
\frac{\partial}{\partial t}\left(\rho^{\lambda} \varepsilon_{2}^{\lambda}\right)+\nabla \cdot\left(\rho \varepsilon_{2}^{\lambda} \mathbf{v}^{\lambda}\right) & =-\lambda \rho^{\lambda}\left(\varepsilon_{2}^{\lambda}-\Phi\left(\rho^{\lambda}, \varepsilon_{1}^{\lambda}\right)\right),
\end{aligned}\right.
$$

with the total energy density

$$
e_{1}^{\lambda}=\rho\left(\varepsilon_{1}^{\lambda}+\frac{\left|\mathbf{v}^{\lambda}\right|^{2}}{2}\right),
$$


the polytropic equation of state $p_{1}^{\lambda}=\left(\gamma_{1}-1\right) \rho \varepsilon_{1}^{\lambda}$ with a constant $\gamma_{1}>1$ and the energy function $\Phi(\rho, \varepsilon)$. It can be shown that in the equilibrium limit $\lambda \rightarrow \infty$ the original Euler equations are obtained:

Theorem 4.9.1. Consider a family of classical solutions

$$
\left(\rho^{\lambda}, \rho^{\lambda} \mathbf{v}^{\lambda}, e_{1}^{\lambda}, \rho^{\lambda} \varepsilon_{2}^{\lambda}\right)_{\lambda>0}
$$

of the relaxation system (4.9.1), with $\left(\rho^{\lambda}, \rho^{\lambda} \mathbf{v}^{\lambda}, e_{1}^{\lambda}\right)^{T} \in \Psi$ as defined in (3.3.2), that is uniformly bounded with respect to $\lambda$. Assume that the equilibrium limit

$$
\mathbf{U}(\mathbf{x}, t):=\lim _{\lambda \rightarrow \infty}\left(\begin{array}{c}
\rho^{\lambda} \\
\rho^{\lambda} \mathbf{v}^{\lambda} \\
e_{1}^{\lambda}+\rho^{\lambda} \varepsilon_{2}^{\lambda}
\end{array}\right)(\mathbf{x}, t)
$$

exists. Then $\mathbf{U}$ is a solution of the Euler equations (3.3.1) if we choose

$$
\Phi\left(\rho, \varepsilon_{1}\right)=\varepsilon\left(\rho, p_{1}\left(\rho, \varepsilon_{1}\right)\right)-\varepsilon_{1},
$$

where $\varepsilon(\rho, \cdot)$ is the inverse of $p(\rho, \cdot): p(\rho, \varepsilon(\rho, \bar{p}))=\bar{p}$ for fixed $\rho$.

For the proof of this theorem cf. [Ded03].

In order to construct the numerical scheme for the relaxation step we need the following

Theorem 4.9.2. Let the energy $\Phi$ be given by (4.9.3) and assume that $\Phi$ is monotone increasing in $\varepsilon_{1}$. Consider the system of ODEs

$$
\left\{\begin{aligned}
\frac{\partial}{\partial t} \rho^{\lambda} & =0 \\
\frac{\partial}{\partial t}\left(\rho^{\lambda} \mathbf{v}^{\lambda}\right) & =0 \\
\frac{\partial}{\partial t} e_{1}^{\lambda} & =\lambda \rho^{\lambda}\left(\varepsilon_{2}^{\lambda}-\Phi\left(\rho^{\lambda}, \varepsilon_{1}^{\lambda}\right)\right), \\
\frac{\partial}{\partial t}\left(\rho^{\lambda} \varepsilon_{2}^{\lambda}\right) & =-\lambda \rho^{\lambda}\left(\varepsilon_{2}^{\lambda}-\Phi\left(\rho^{\lambda}, \varepsilon_{1}^{\lambda}\right)\right),
\end{aligned}\right.
$$

with the initial conditions given by

$$
\left(\rho_{0},(\rho \mathbf{v})_{0},\left(e_{1}\right)_{0},\left(\rho \varepsilon_{2}\right)_{0}\right) .
$$

Denote with $\left(\varepsilon_{1}\right)_{0}$ the internal energy of the initial data defined through the relation (4.9.2). Then the solution of (4.9.4) for $\lambda \rightarrow \infty$ is

$$
\left(\rho_{0},(\rho \mathbf{v})_{0}, \rho_{0}\left(\varepsilon_{1}^{*}+\frac{1}{2}\left|\mathbf{v}_{0}\right|^{2}\right), \rho_{0} \varepsilon_{2}^{*}\right) .
$$

The constants $\varepsilon_{1}^{*}$ and $\varepsilon_{2}^{*}$ are defined by the algebraic relations

$$
\begin{aligned}
p\left(\rho_{0},\left(\varepsilon_{1}\right)_{0}+\left(\varepsilon_{2}\right)_{0}\right) & =p_{1}\left(\rho_{0}, \varepsilon_{1}^{*}\right), \\
\varepsilon_{1}^{*}+\varepsilon_{2}^{*} & =\left(\varepsilon_{1}\right)_{0}+\left(\varepsilon_{2}\right)_{0} .
\end{aligned}
$$


If $p_{1}\left(\rho, \varepsilon_{1}\right)=\left(\gamma_{1}-1\right) \rho \varepsilon_{1}$ then $\varepsilon_{1}^{*}$ and $\varepsilon_{2}^{*}$ are given by the explicit relations

$$
\begin{aligned}
\varepsilon_{1}^{*} & :=\frac{p\left(\rho_{0}, \varepsilon_{0}\right)}{\left(\gamma_{1}-1\right) \rho_{0}} \\
\varepsilon_{2}^{*} & :=\varepsilon_{0}-\varepsilon_{1}^{*}
\end{aligned}
$$

with $\varepsilon_{0}:=\left(\varepsilon_{1}\right)_{0}+\left(\varepsilon_{2}\right)_{0}$.

The proof of this theorem can also be found in [Ded03].

\subsubsection{The Numerical Scheme}

Now in order to use our initial AUSMDV Riemann solver the following scheme is constructed. For given left and right-hand states $\mathbf{U}_{l / r}=\left(\rho_{l / r},(\rho \mathbf{v})_{l / r}, e_{l / r}\right)^{T}$ we construct the numerical flux $\mathbf{g}_{j l}\left(\mathbf{U}_{l}, \mathbf{U}_{r}\right)$ consisting of a relaxation step, the employment of the standard Euler flux $\mathbf{g}_{j l}^{\text {perf }}\left(\tilde{\mathbf{U}}_{l}, \tilde{\mathbf{U}}_{r}\right)$, followed by a pure advection of the remaining internal energy $\varepsilon_{2}$. The solution of the relaxation step can be analytically defined by using Theorem 4.9.2 on the page before

$$
\begin{aligned}
\varepsilon_{2, l / r} & :=\Theta_{2}\left(\mathbf{U}_{l / r}\right):=\varepsilon_{l / r}-\frac{p_{l / r}\left(\rho_{l / r}, \varepsilon_{l / r}\right)}{\rho_{l / r}\left(\gamma_{1, l / r}-1\right)}, \\
\tilde{\mathbf{U}}_{l / r} & :=\boldsymbol{\Theta}_{1}\left(\mathbf{U}_{l / r}\right):=\left(\rho_{l / r},(\rho \mathbf{v})_{l / r}, e_{l / r}-\rho_{l / r} \varepsilon_{2, l / r}\right)^{T} .
\end{aligned}
$$

The constant $\gamma_{1, l / r}$ has to fulfill the additional restriction

$$
\gamma_{1, l / r}>\max \left\{\gamma\left(\rho_{l}, \varepsilon_{l}\right), \gamma\left(\rho_{r}, \varepsilon_{r}\right), \Gamma\left(\rho_{l}, \varepsilon_{l}\right), \Gamma\left(\rho_{r}, \varepsilon_{r}\right)\right\}
$$

with $\gamma(\rho, \varepsilon):=\frac{\rho c^{2}(\rho, \varepsilon)}{p(\rho, \varepsilon)}, \Gamma(\rho, \varepsilon):=1+\frac{1}{\rho} \partial_{\varepsilon} p(\rho, \varepsilon)$ (as shown in [Ded03]). However, in our case the definition of $\Gamma(\rho, \varepsilon)$ reduces to

$$
\Gamma(\rho, \varepsilon)=1+\frac{R}{c_{\mathrm{V}}(T)}=\gamma(T)=\gamma(\rho, \varepsilon)
$$

(see Paragraph 3.2.2 on page 29), resulting in the attenuated restriction

$$
\gamma_{1, l / r}>\max \left\{\gamma\left(\rho_{l}, \varepsilon_{l}\right), \gamma\left(\rho_{r}, \varepsilon_{r}\right)\right\}
$$

Now the convective numerical flux function $\mathbf{g}_{j l}^{\text {perf }}\left(\tilde{\mathbf{U}}_{l}, \tilde{\mathbf{U}}_{r}\right)$, i.e. the AUSMDV Riemann solver, can be used to compute the fluxes for $\tilde{\mathbf{U}}=\left(\rho, \rho \mathbf{v}, e_{1}\right)^{T}$.

Then the advection is calculated with the help of the mass flux approximation $\left(\mathbf{g}_{j l}^{\text {perf }}\right)_{1} \approx \rho \mathbf{v}$ on face $S_{j l}$. In the case that it is positive $\varepsilon_{2}$ should be advected to the left otherwise to the right:

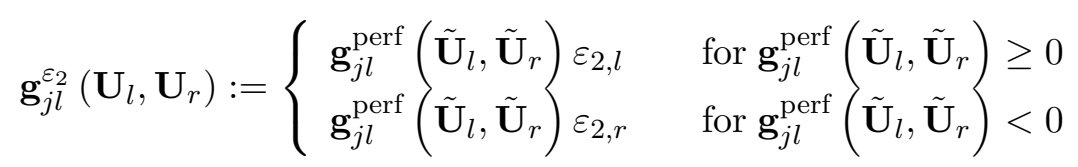


Thus, the convective numerical flux according to the energy relaxation scheme is given by

$$
\mathbf{g}_{j l}\left(\mathbf{U}_{l}, \mathbf{U}_{r}\right):=\mathbf{g}_{j l}^{\text {perf }}\left(\tilde{\mathbf{U}}_{l}, \tilde{\mathbf{U}}_{r}\right)+\left(0, \mathbf{0}, \mathbf{g}_{j l}^{\varepsilon_{2}}\left(\mathbf{U}_{l}, \mathbf{U}_{r}\right)\right) .
$$

For a detailed description and analysis of this method see [Ded03]. It is also shown that a higher efficiency is attained by using this technique as opposed to the standard FVS.

The application of this relaxation scheme to the extended finite volume scheme that accounts for the two gas mixture species fresh-gas and exhaust-gas (see Paragraph 3.7.3 on page 38) is straightforward.

\subsubsection{Tabularized Equation of State}

To calculate the pressure $p$ from the vector $\mathbf{U}$ of conservative variables, the temperature $T$ has to be evaluated from the calorical equation of state (3.5.2). As this temperature is only given implicitly, a tabularized version of this equation is computed, thus making $T(\varepsilon)$ available. As the heat capacity at constant volume $c_{\mathrm{V}}(T)$ is assumed to be constant for $n \leq T<n+1$, for $n \in \mathbb{Z}$, this table is easy to compute. The step size is chosen as $1000 \mathrm{~J}$. This corresponds approximately to the one degree-step size of the table $\varepsilon(T)$. These step sizes have been chosen according to the slope of the functions under consideration.

With this temperature $T$ and the given density $\rho$ one can then easily evaluate the thermal equation of state (3.5.1) in order to obtain the pressure $p$.

\subsection{The Algorithm of the Main Numerical Scheme}

Summing up the last chapter, in Flowchart 4.10.1 on the next page we present the algorithm of the main numerical scheme. This algorithm is completed part by part in the following chapters. 


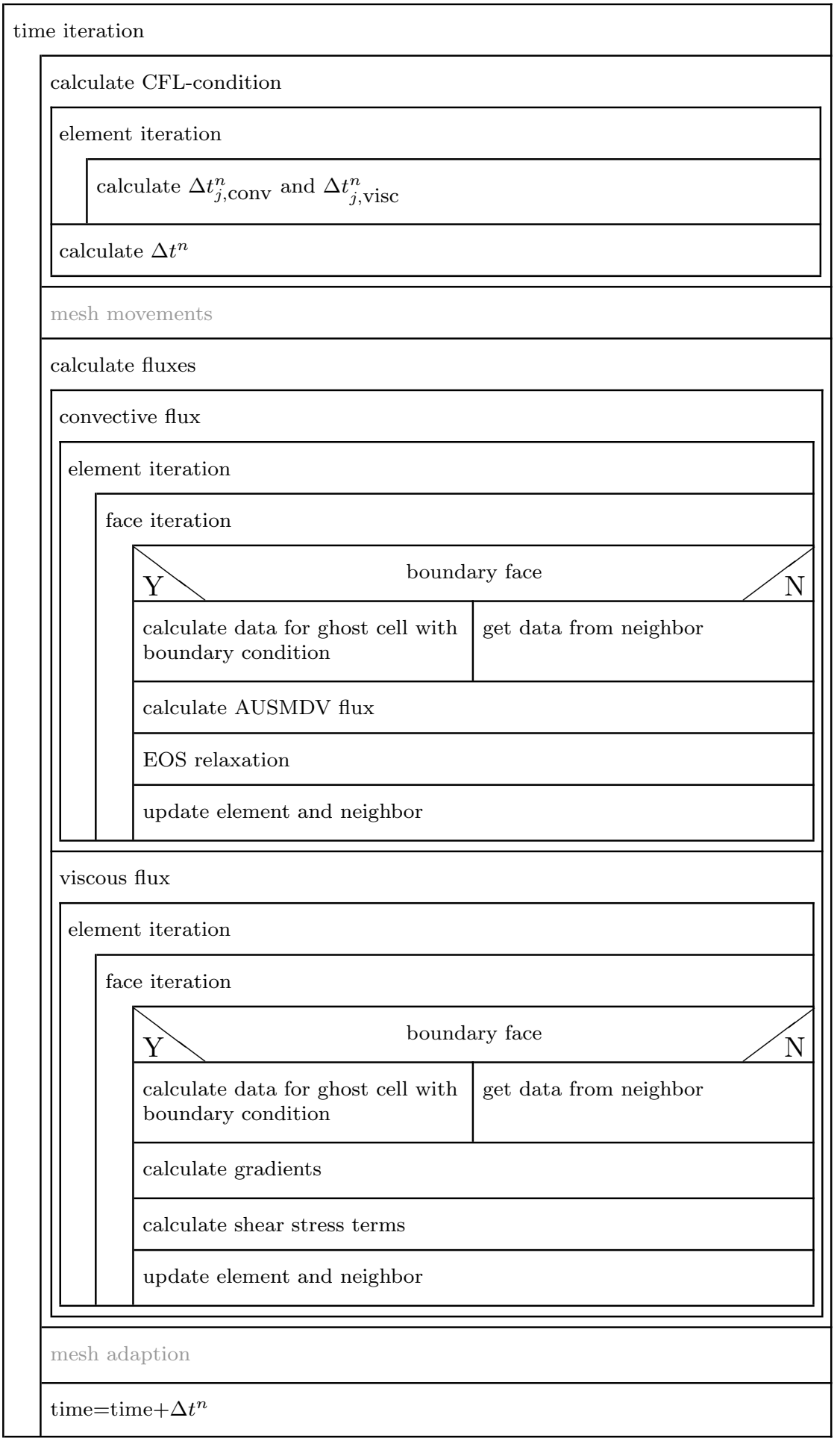

Figure 4.10.1: Flowchart of the main numerical scheme. 


\section{Chapter 5}

\section{Special Mesh Treatment}

\subsection{Grid Merging}

As mentioned in Remark 4.2.5 on page 47, we use meshes consisting of hexahedrons. But this kind of mesh is more difficult to generate than tetrahedral meshes, especially for complex geometries in three space dimensions. Therefore, it is usually a great benefit to assemble a mesh from different parts and "glue together" these composite meshes on their interfaces. The advantages are the facilitation of the mesh generation and the better quality of the resulting mesh due to less topological constraints on the interfaces of the different building blocks and the absence of restrictions to the shape of the ports that connect to the cylinder and the crankcase (for details refer to Section 10.1 on page 167).

\subsubsection{Different Techniques}

\section{The Overlapping Grid Scheme}

There are two main ideas to approach the problem of assembling grids. One is the overlapping (or overset) grid scheme. As shown on the left-hand side of Figure 5.1.1 on the following page, we have in this case two or more grids that overlap. On each grid the system of partial differential equations is solved. The values on boundary points are obtained by an interpolation procedure from the data on inner points of the other grid (see [Fuj95, SB87]). Sometimes holes have to be cut out of a mesh in order to exclude these elements from the flow field calculation as described in [Pet99]. The big drawback of this method of overlapping grids is its non-conservativeness if no special precautions are undertaken. The conservation property, as set forth in the numerical scheme (see Section 4.3.2 on page 49), is very important, e.g. in order to capture shock waves passing through the boundary. For maintaining the conservation it is necessary to solve an additional system of equations to balance the interpolation coefficients (cf. [CH94]). This computational effort is avoided by using the second approach described now. 


\section{The Patched Grid Scheme}

Here, the different parts of the mesh are constructed so that they share common boundary interfaces. But still the single element faces usually join discontinuously as shown in Figure 5.1.1 (right). In this situation it is possible to avoid the need for interpolation of data from the different grids. The interfaces are split into several facets and the flux over each facet is calculated and accounted for on either side of the facet. Therefore, we preserve the conservation property over the mesh interface without the need for any additional computation. The only effort has to be put into the calculation of the facets between two elements of the two adjacent meshes. This is described now in further detail.
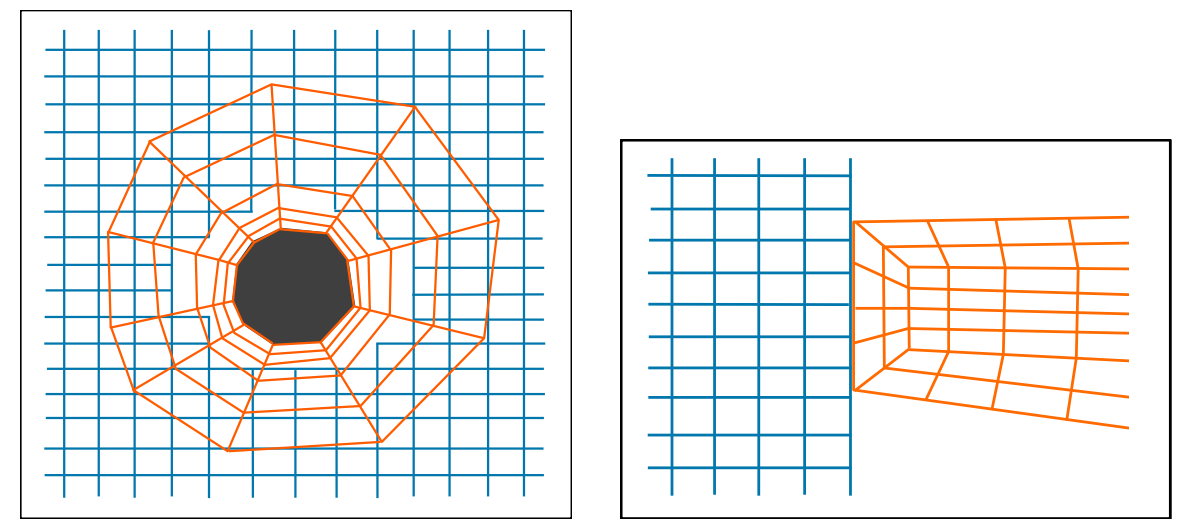

Figure 5.1.1: Overset (left) and patched (right) grids.

\subsubsection{Matching Interfaces with the Patched Grid Scheme}

As mentioned before, the faces of the boundary elements of the two meshes under consideration generally do not match each other. Thus, a direct calculation of the fluxes is not possible. Our approach now consists in splitting up the face $S_{j l}$ of element $T_{j}$ into several facets $S_{j l k}$ such that each of these facets is shared by two neighboring elements $T_{j}$ and $T_{j l k}$ for $0 \leq k \leq k_{j l}$ with $k_{j l}$ being the number of neighbors of element $T_{j}$ over the boundary face $S_{j l}$ (as sketched on the left-hand side of Figure 5.1.2 on page 68). With this method an element therefore not only has one neighbor $T_{j l}$ per face $S_{j l}$ anymore but $k_{j l}$ neighbors. In order to evaluate the corresponding numerical convective and viscous fluxes $g_{j l k}$ and $G_{j l k}$ of these two elements $T_{j}$ and $T_{j l k}$ through their common facet $S_{j l k}$, the only additional information we need to calculate is the area of the common facet $S_{j l k}$. However, apart from this modification the standard scheme as described in Chapter 4 on page 45 can be applied. As the neighbors $T_{j l k}$ and the area of the corresponding common facets $S_{j l k}$ can be calculated beforehand and saved in a special structure (see the next Paragraph 5.1.3 on the facing page), this method needs very little computational time (as shown in Paragraph 5.1.4 on the next page). 


\subsubsection{The Structure}

When the calculation of the numerical fluxes reaches a face on an inter-mesh interface a special treatment of this face is necessary. Thus, we need a new boundary condition as an indicator of such an interface.

Then, in order to calculate these fluxes, the area of each facet $S_{j l k}$, its outer normal $\mathbf{n}_{j l k}$, and the pointer to the corresponding neighbor $T_{j l k}$ are used. Therefore, this information has to be stored in the grid merging structure in the initialization phase and can then be used at each iteration step. As the number of neighbors $k_{j l}$ of element $T_{j}$ on such an interface is not bounded, a linked list is employed.

\subsubsection{The Algorithm}

\section{Initialization}

In the initialization phase the neighbors of each inter-mesh boundary element $T_{j}$ have to be detected. For the first neighbor $T_{j l 0}$ a heuristic approach is used. It is assumed that the connecting faces have a similar size. Then we want to find a boundary element $T_{m}$ from a different part of the mesh that satisfies

$$
\left|\mathbf{z}_{j l}-\mathbf{z}_{m n}\right|^{2}<2\left|S_{j l}\right|
$$

where $\mathbf{z}_{j l}$ is the center of gravity of $S_{j l}$. If such an element is found it is used as a starting point for an iterative depth search of all the neighboring elements of $T_{j}$. Depth level $d=0$ is the starting element itself, elements of depth level $d=1$ are the neighbors of the starting element, elements of level $d=2$ are the neighbors of these neighbors, and so on. The common area $A_{j l k}$ of the boundary faces of these elements with face $S_{j l}$ is calculated. As this area calculation is a $2 \mathrm{D}$ problem, it can be handled by fast 2D algorithms to decide if a point is inside a face, to calculate the intersection point of two line segments, to sort vectors, and finally, to calculate the area $A_{j l k}$ of the common facet. If $A_{j l k}>0$ a facet $S_{j l k}$ is created and the grid merge structure is filled with this information. The recursive search is stopped if there are no new facets $S_{j l k}$ when advancing from depth level $d$ to depth level $d+1$. If

$$
\sum_{k=0}^{k_{j l}}\left|S_{j l k}\right|=\left|S_{j l}\right|
$$

then element $T_{j}$ is an inner element and all neighbors over boundary face $S_{j l}$ have been found. Otherwise, element $T_{j}$ is situated at a corner of the composite mesh and a part of the face $S_{j l}$ is a boundary as depicted in Figure 5.1.2 (right).

\section{Time Iteration}

On each face $S_{j l}$ of each element $T_{j}$ the fluxes are calculated as described in Chapter 4 on page 45 . If a boundary to another part of the mesh is encountered, each facet $S_{j l k}$ of face $S_{j l}$ is handled in turn in the same manner as a normal face. Only $\left|S_{j l}\right|$ is replaced by $\left|S_{j l k}\right|, \mathbf{n}_{j l}$ by $\mathbf{n}_{j l k}$, and $T_{j l}$ by $T_{j l k}$. If

$$
A_{\mathrm{bnd}}:=\left|S_{j l}\right|-\sum_{k=0}^{k_{j l}}\left|S_{j l k}\right|>0,
$$


i.e. we are in the case of Figure 5.1.2 (right), then the residual face of area $A_{\text {bnd }}$ is calculated as a wall boundary condition with convective numerical flux

$$
g_{j l k}=\left(\begin{array}{c}
0 \\
p \mathbf{n}_{j l} \\
0
\end{array}\right)
$$

and viscous numerical flux $G_{j l}$ as described in Sections 4.5 on page 52 and 4.7 on page 55 .

This algorithm is summed up in the Flowchart 5.1.4 on the next page.
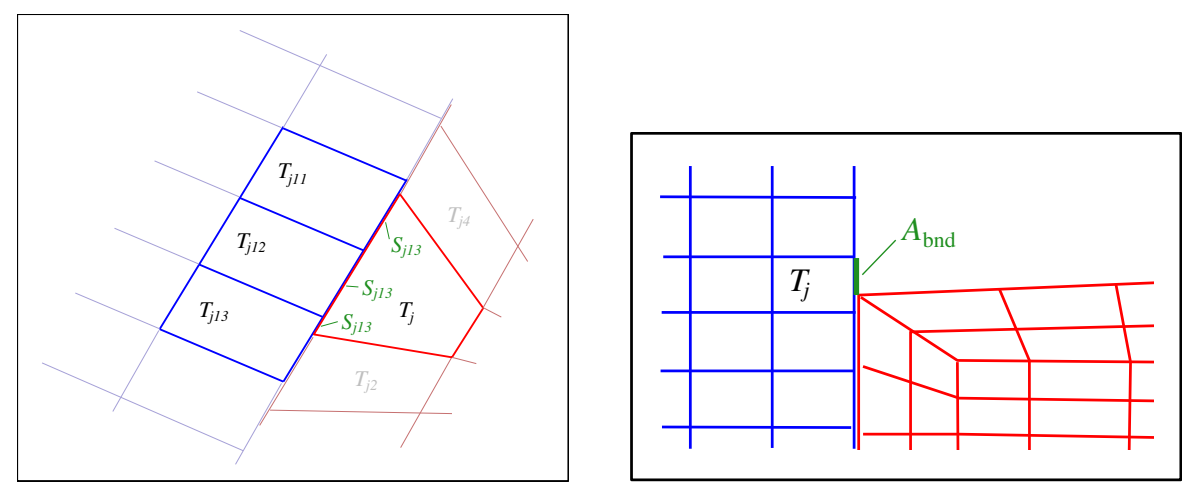

Figure 5.1.2: Detailed view of a patched grid (left) and boundary facet (right).

\subsubsection{The Massflow Problem}

With this treatment of the boundary elements (where $A_{\mathrm{bnd}}>0$ ) a correct massflow through such an interface is not achieved (see test case 9.4.1 on page 145). In Figure 5.1.3 this situation is sketched. The impulse is conserved at the transition but in element $T$ in Figure 5.1.3 (left) this impulse is distributed throughout the whole volume, thus slowing down the flow. With a perfectly matching grid, this effect would not be observed (Figure 5.1.3 (middle)). The flow continues undamped into the larger volume.
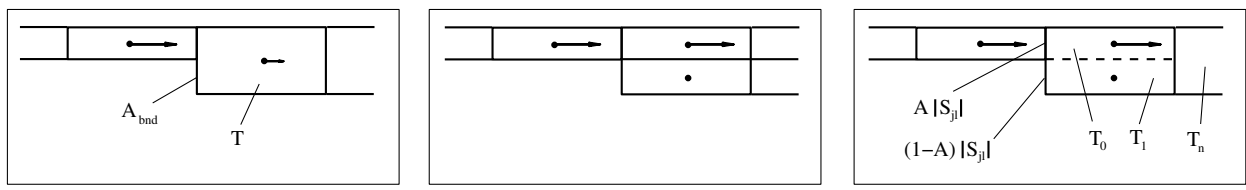

Figure 5.1.3: The virtual splitting method: without splitting (left), $A_{\mathrm{bnd}}=0$ no splitting necessary (middle), and with virtual splitting (right). 


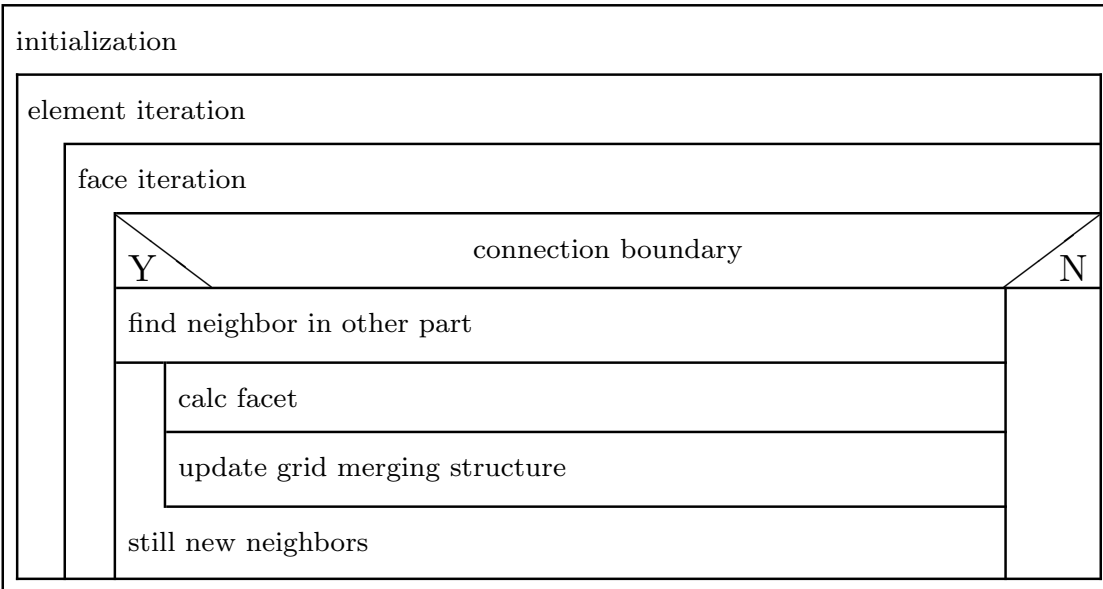

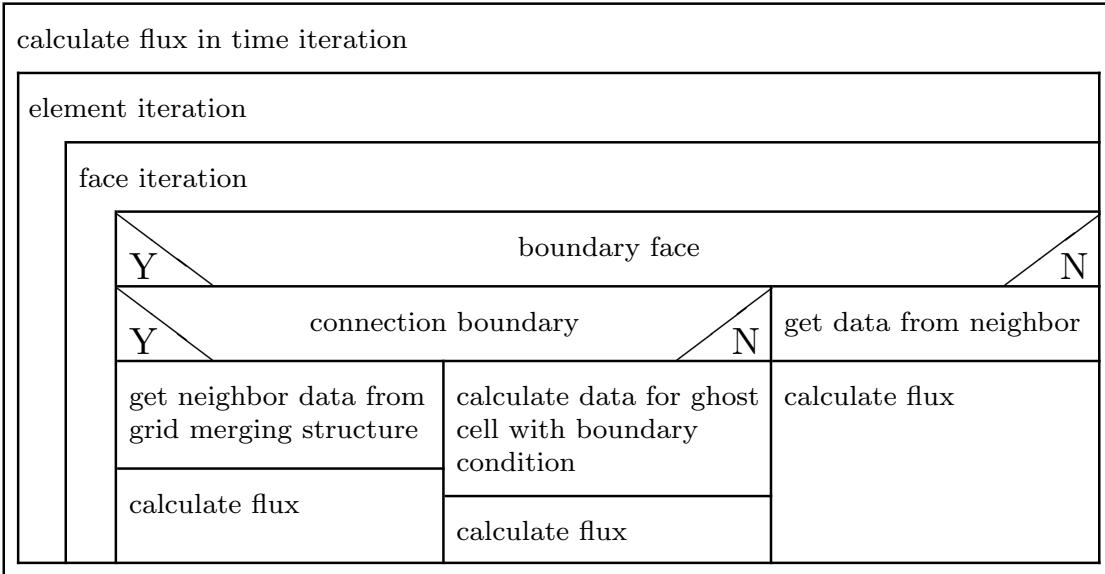

Figure 5.1.4: Flowchart of the grid merging algorithm. 


\section{The Virtual Splitting Method}

In order to simulate this behavior, a new treatment of these boundary cells has been devised. The element $T$ in Figure 5.1.3 (left) is virtually treated as two elements $T_{0}$ and $T_{1}$ as depicted in Figure 5.1.3 (right). And depending on the percentage of area $A:=\frac{1}{\left|S_{j l}\right|}\left(1-A_{\mathrm{bnd}}\right)$, the velocity is shifted into the connecting part of the virtual cell. Attention is paid to the conservation principle of mass, momentum, and energy. With $\mathbf{U}_{01}=\left(\rho_{01}, \rho_{01} v_{01}, e_{01}\right)$ being the values of the cell $T$ before the virtual split and $\mathbf{U}_{0}$ and $\mathbf{U}_{1}$ the values of the connected and boundary cell $T_{0}$ and $T_{1}$ respectively, we have the conservation constraints:

$$
\begin{aligned}
\rho_{01} & =A \rho_{0}+(1-A) \rho_{1} \\
\rho_{01} v_{01} & =A \rho_{0} v_{0}+(1-A) \rho_{1} v_{1} \\
e_{01} & =A e_{0}+(1-A) e_{1}
\end{aligned}
$$

According to the idea to shift the velocity into the virtual cell $T_{0}, v_{1}$ is set to zero:

$$
v_{1}:=0
$$

A further necessary condition for conservation is given by:

$$
\sum_{l=1}^{k_{j}} p \mathbf{n}_{j l}=0
$$

If we also calculate the neighboring cell $T_{n}$ with the values of the virtual split cell this translates, in our context (with the notations of Figure 5.1.3), to:

$$
-\mathbf{n}_{j l}\left(A p_{0}+(1-A) p_{1}\right)+\mathbf{n}_{j l}\left(A p_{0}+(1-A) p_{1}\right)=0
$$

which is obviously true. (By calculating the cell $T_{n}$ with the original values $\mathbf{U}_{01}$, this condition would render the system of constraints unsolvable.)

Lemma 5.1.1. (Solution of the virtual split) The following set of split values satisfies the above-stated constraints (5.1.2) and (5.1.3):

$$
\begin{aligned}
\rho_{0} & =\rho_{1}=\rho_{01} \\
v_{0} & =\frac{1}{A} v_{01} \\
e_{0} & =e_{01}+\frac{1-A}{2 A^{2}} \rho_{01} v_{01}^{2} \\
e_{1} & =e_{01}-\frac{1}{2 A} \rho_{01} v_{01}^{2}
\end{aligned}
$$

These values further satisfy:

$$
p_{0}=p_{1}
$$

with the pressure $p$ given by the equation of state for a calorically perfect (as in (3.5.3)) or imperfect gas (as in (3.5.2)).

Proof. This is a straightforward calculation. 
The problem that is encountered in these values is the dependency of $v_{0}$ on $\frac{1}{A}$. For $A \rightarrow 0$ we have $v_{0} \rightarrow \infty$ if $v_{01} \neq O(A)$. In numerical tests condition $v_{01} \neq O(A)$ was encountered. Therefore, we need to construct a transition solution for $0<A<\varepsilon$. This is the purpose of the next Lemma.

Lemma 5.1.2. (Solution of the virtual split for $A<\varepsilon$ ) Under the constraints (5.1.2) and the boundary values

$$
\begin{aligned}
& \text { for } A=0:\left\{\begin{array}{l}
\rho_{0}=\rho_{1}=\rho_{01} \\
v_{0}=v_{1}=v_{01} \\
p_{0}=p_{1}=p_{01}
\end{array}\right. \\
& \text { for } A=\varepsilon:\left\{\begin{array}{l}
\rho_{0}=\rho_{1}=\rho_{01} \\
v_{0}=\frac{1}{\varepsilon} v_{01} \\
v_{1}=0 \\
p_{0}=p_{1}
\end{array}\right.
\end{aligned}
$$

the following split values for small $A$ are admissible:

$$
\begin{aligned}
\rho_{0} & =\rho_{1}=\rho_{01} \\
v_{0} & =\left(\frac{A}{\varepsilon}\left(\frac{1}{\varepsilon}-1\right)+1\right) v_{01} \\
v_{1} & =\frac{1}{1-A} v_{01}-\frac{A}{1-A} v_{0} \\
e_{0} & =e_{01}+(1-A) \frac{\rho_{01}}{2}\left(v_{0}^{2}-v_{1}^{2}\right) \\
e_{1} & =e_{01}-A \frac{\rho_{01}}{2}\left(v_{0}^{2}-v_{1}^{2}\right)
\end{aligned}
$$

Proof. This Lemma is also verified by an easy calculation.

In three dimensions there might also be velocities in the $y$ - and $z$-direction. These velocities are not touched by our virtual splitting:

$$
\begin{aligned}
& \left(v_{0}\right)_{y}=\left(v_{1}\right)_{y}=\left(v_{01}\right)_{y} \\
& \left(v_{0}\right)_{z}=\left(v_{1}\right)_{z}=\left(v_{01}\right)_{z}
\end{aligned}
$$

In both virtual cells these velocities are the original ones.

\section{The Algorithm}

If, in our numerical scheme, we encounter an element $T_{j}$ which has the property $A_{\text {bnd }}>0$ then two numerical fluxes are calculated. One flux uses the split value $\mathbf{U}_{0}$ and the face volume $\left|S_{j l}^{\text {con }}\right|:=A\left|S_{j l}\right|$ and the other flux the split values $\mathbf{U}_{1}$ and $\left|S_{j l}^{\text {bnd }}\right|:=(1-A)\left|S_{j l}\right|$. The split values $\mathbf{U}_{0}$ and $\mathbf{U}_{1}$ are obtained by using Lemma 5.1.1 on the facing page or Lemma 5.1.2 depending on $A<\varepsilon$ or $A>\varepsilon$, respectively. 


\subsection{The Piston Motion}

The vertical position of the piston is determined by an explicit formula stated in Lemma 2.3.10 on page 13. In order to realize this position the mesh has to be changed dynamically after each iteration step. This mesh changing affects also the connection to other parts of the grid, namely the transfer ports, the inlet and the outlet duct. This dynamic changing has to be applied in the cylinder as well as in the crankcase. In the crankcase we have the additional problem that the piston bottom is not a flat surface but has extensions that are necessary for a correct timing of the inlet duct opening.

\subsubsection{Different Techniques}

For this dynamic piston motion several methods of mesh changing have been proposed. Some of these are described here.

\section{Grid Compression}

A first approach is to compress and stretch the whole grid as the piston moves up and down, called ICED-ALE ([Hir74]), as applied e.g. in [PHPM88] for unstructured meshes. But, as the compression ratio in two-stroke engines is usually very high, this would lead to many very small cells as the piston reaches tdc, decreasing the time step dramatically.

\section{Re-Meshing}

To solve this problem one could re-mesh the area under consideration with bigger cells. The data on these new cells would be obtained by interpolation of the old mesh (see e.g. [Hir74, PHPM88]). But still the whole grid must be moved in each step, which is computationally costly, and an additional interpolation from the old to the new mesh during the re-meshing process can be very complicated and timeconsuming and introduces a further interpolation error. Furthermore, the generation of a new grid might not be that easy for the irregular lower surface of the piston.

\section{The Collected Cell Algorithm}

With this approach, a cutting technique (cf. [Wie95]) intersects all elements with the piston crown. Only the part of the element above the piston is retained. If an element happens to be too small (and the CFL condition would imply too small a time step) it is merged with other neighboring cells by the collected cell algorithm. This method results in complex case differentiation and many different types of elements, depending on the orientation of the element that has been cut by the piston, even more so if the piston is not flat anymore. However, the advantage is that an arbitrary tetrahedral mesh can be used to discretize the cylinder and crankcase. In addition, the calculation of less and less elements in the cylinder is necessary as the piston moves its way upwards, reducing computational time for the flow simulation. 


\section{The Snapper Algorithm}

The requirement of the snapper algorithm (see e.g. [ARB92]) is an equal distribution of hexahedrons or prisms oriented parallel to the piston surface. If the piston moves, e.g. upwards, the lowest layer of elements in the cylinder (above the piston) is reduced in size as long as this does not result in too small elements. If too small elements occur the lowest layer of elements is merged with the next upper one, which now becomes the lowest layer. This process is reversed in the case of the downwards motion of the piston. The elements in the crankcase (below the piston) are treated in an analogous manner. The advantage of this algorithm is its easy application and therefore its low cost with respect to computational time. And as with the Collected Cell Algorithm there are always many layers of deactivated cells, which can therefore be neglected for the flow calculation.

\subsubsection{Detailed Description of the Snapper Algorithm}

Because we decided to use a hexahedral mesh (see Remark 4.2.5 on page 47), the snapper algorithm is a natural choice. It is not difficult to assure that we have a prescribed parallel orthogonal layering within the lower part of the cylinder and the upper part of the crankcase.

In the initialization step we first build a list of all layers in the cylinder and crankshaft to grant a fast and easy access to all elements of the lowest and second lowest layer at all times. Within the time iteration the exact position $h_{\text {new }}=h_{p}(\Theta)$ of the piston above bdc is calculated via the explicit formula of Lemma 2.3.10 on page 13. This new position $h_{\text {new }}$ is compared to the old position $h_{\text {old }}$. For the following description we assume an upward motion of the piston, i.e $h_{\text {new }}>h_{\text {old }}$. Therefore, above the piston the elements of the lowest layer have to shrink. Their lower face rises to position $h_{\text {new }}$ and identifies the new boundary to the piston crown (see the upper part of Figure 5.2.1 on the following page) if their new relative height $l_{\text {rel }}$ is not smaller than a certain prescribed threshold $t$, where $l_{\text {rel }}$ is defined as

$$
l_{\mathrm{rel}}:=\frac{l_{\mathrm{act}}}{l_{\mathrm{orig}}}:=\frac{h_{\mathrm{u}}-h_{\mathrm{l}, \mathrm{act}}}{h_{\mathrm{u}}-h_{\mathrm{l}, \mathrm{orig}}},
$$

with relative height $l_{\text {rel }}$ of the lowest layer, actual height $l_{\text {act }}$, original height $l_{\text {orig }}, h_{\mathrm{u}}$ position of the upper face, $h_{1, \text { act }}$ the actual position of the lower face, and $h_{1 \text {,orig }}$ the original position of the lower face. The data in these cells is then adapted according to the conservation of mass, impulse and energy:

$$
u_{\text {new }}=\frac{V_{\text {old }}}{V_{\text {new }}} u_{\text {old }},
$$

where $u$ is the conserved variable $\rho, \rho \mathbf{v}$, and $e$.

If $l_{\text {rel }}<t$, i.e. the new cells would be too small, the lowest layer "snaps" back to its original position, is deactivated, and the lower face of the elements of the former second lowest layer is set to position $h_{\text {new }}$. The boundary condition of these faces is changed in order to identify the piston boundary (Figure 5.2.1 (middle)). Now the data in the cells has to be matched to fulfill the conservation properties:

$$
u_{\text {new }}=\frac{V_{\mathrm{l}, \mathrm{old}}}{V_{\text {new }}} u_{1, \text { old }}+\frac{V_{\mathrm{s}, \text { old }}}{V_{\text {new }}} u_{\mathrm{s}, \text { old }}
$$


with $(\cdot)_{1, \text { old }}$ and $(\cdot)_{\mathrm{s}, \mathrm{old}}$ the former value of the element in the lowest and second lowest layer, respectively.

In the case of downward motion of the piston the faces of the lowest elements are lowered to the new position $h_{\text {new }}$. The data on the elements is adjusted in the same manner as in the compression of the cells. The elements are split into two layers if the height of the new layer is larger than the threshold percentage of its original size. In this case the elements of the new layer are activated, the boundary condition is shifted down one layer, the now second lowest layer is restored to its original size, and the lower face of the just activated elements is moved to represent the piston position $h_{\text {new }}$ (as displayed in Figure 5.2.1 (bottom)). Also the data in the cells is split into the two new layers:

$$
u_{1, \text { new }}=u_{\mathrm{s}, \text { new }}=\frac{V_{\text {old }}}{V_{\text {l,new }}+V_{\mathrm{s}, \text { new }}} u_{\text {old }}
$$
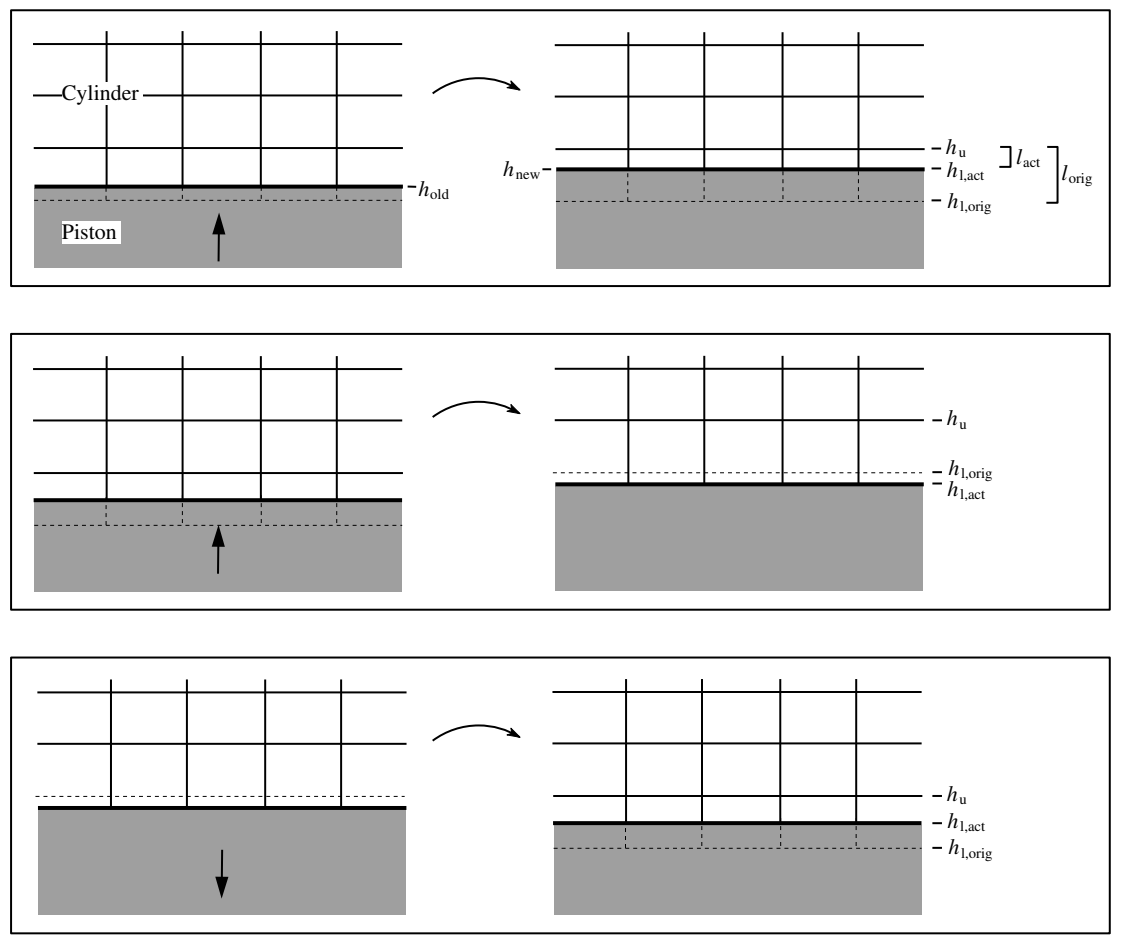

Figure 5.2.1: The snapper algorithm, moving (top), merging (middle), and splitting (bottom).

\subsubsection{The Extended Snapper Algorithm}

With the above described approach the piston surface does not need to be flat as long as a layering of the elements along the piston surface is possible. However, the bottom side of the piston does not allow for this layering as one can see in Figure 5.2.2 on the next page. Therefore, the snapper algorithm has to be extended 
to this case. On the upper part of the mesh (area $a$ on Figure 5.2.2) the standard algorithm can be applied. Just on the lower part of the mesh (area $b$ on Figure 5.2.2) special care has to be taken of the neighboring cells, which are affected by the motion of the upper faces of the elements at the piston boundary (as seen in Figure 5.2.3 on the following page).

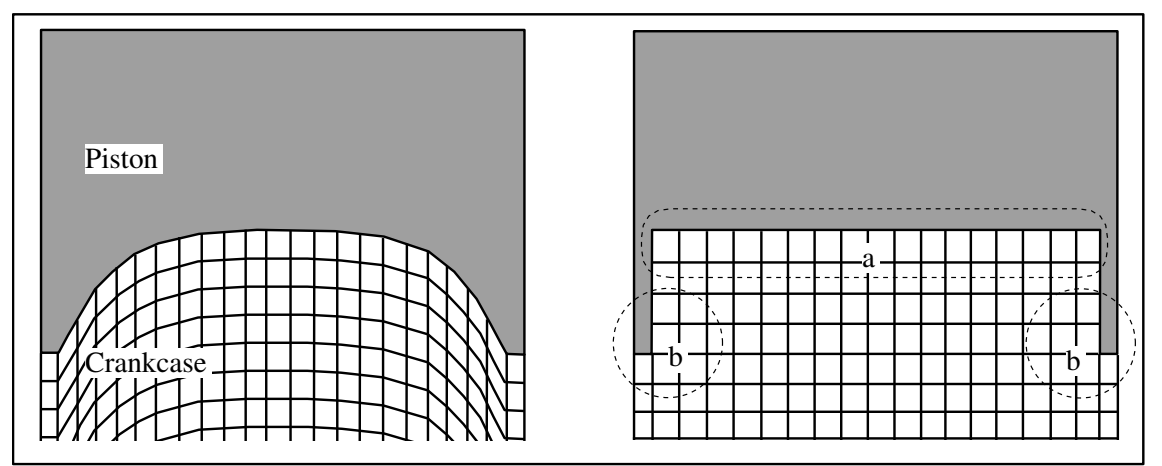

Figure 5.2.2: Two different pistons: layering possible (left) and impossible (right).

We have to deal with three cases to handle the data in these neighbors:

- The lowest layer elements are only changed in size, no merging or splitting occurs (case $a$ in Figure 5.2.3 on the next page):

$$
\begin{aligned}
u_{\mathrm{u}, \text { new }} & =\frac{V_{\mathrm{u}, \text { old }}}{V_{\mathrm{u}, \text { new }}} u_{\mathrm{u}, \text { old }}+\frac{V_{\mathrm{u}, \text { new }}-V_{\mathrm{u}, \text { old }}}{V_{\mathrm{u}, \text { new }}} u_{\mathrm{l}, \text { old }} \\
u_{\mathrm{l}, \text { new }} & =u_{\mathrm{l}, \text { old }}
\end{aligned}
$$

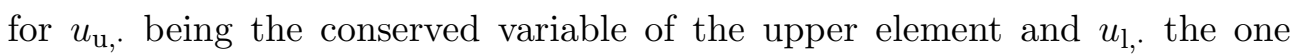
for the lower element ${ }^{1}$. These equations, as well as the ones stated below, result from the transport flux from the lower element to the upper element to compensate for the grid motion.

- The lowest and second lowest layers are merged while the piston moves downwards (case $b$ in Figure 5.2.3):

$$
\begin{aligned}
u_{\mathrm{u}, \text { new }} & =u_{\mathrm{u}, \text { old }} \\
u_{\mathrm{m}, \text { new }} & =\frac{V_{\mathrm{m}, \text { old }}+V_{\mathrm{l}, \mathrm{old}}-V_{\mathrm{l}, \text { new }}}{V_{\mathrm{m}, \text { new }}} u_{\mathrm{m}, \text { old }}+\frac{V_{\mathrm{u}, \mathrm{old}}-V_{\mathrm{u}, \text { new }}}{V_{\mathrm{m}, \text { new }}} u_{\mathrm{u}, \mathrm{old}} \\
u_{\mathrm{l}, \text { new }} & =\frac{V_{\mathrm{l}, \mathrm{old}}}{V_{\mathrm{l} \text { new }}} u_{\mathrm{l}, \text { old }}+\frac{V_{\mathrm{l}, \mathrm{old}}-V_{\mathrm{l}, \text { new }}}{V_{\mathrm{l} \text {,new }}} u_{\mathrm{m}, \text { old }}
\end{aligned}
$$

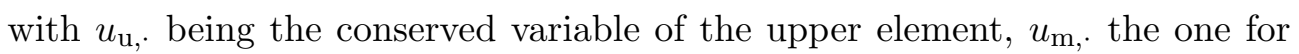
the middle element, and $u_{l}$, for the lower element.

\footnotetext{
${ }^{1}$ Here we stated the equations governing the downward motion of the piston. The upward motion is handled in the same manner.
} 
- The lowest level is split into two layers on the upward motion of the piston (case $c$ in Figure 5.2.3):

$$
\begin{aligned}
u_{\mathrm{u}, \text { new }} & =\frac{V_{\mathrm{u}, \text { old }}}{V_{\mathrm{u}, \text { new }}} u_{\mathrm{u}, \text { old }}+\frac{V_{\mathrm{u}, \text { new }}-V_{\mathrm{u}, \text { old }}}{V_{\mathrm{u}, \text { new }}} u_{\mathrm{m}, \text { old }} \\
u_{\mathrm{m}, \text { new }} & =\frac{V_{\mathrm{u}, \text { old }}+V_{\mathrm{m}, \text { old }}-V_{\mathrm{u}, \text { new }}}{V_{\mathrm{m}, \text { new }}} u_{\mathrm{m}, \text { old }}+\frac{V_{\mathrm{l}, \text { old }}-V_{\mathrm{l}, \text { new }}}{V_{\mathrm{m}, \text { new }}} u_{\mathrm{l}, \text { old }} \\
u_{\mathrm{l}, \text { new }} & =u_{\mathrm{l}, \text { old }}
\end{aligned}
$$

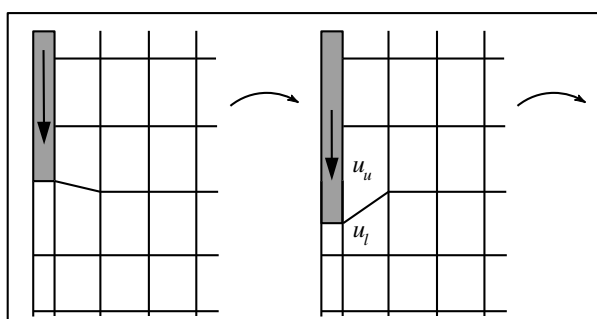

(a) move

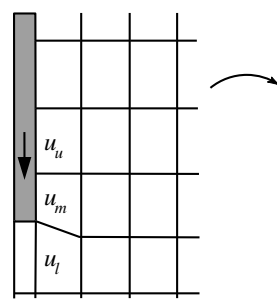

(b) merge

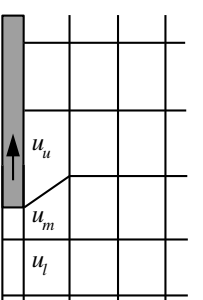

(c) split

Figure 5.2.3: The neighboring cells get affected by the piston motion.

\subsubsection{The Update of the Window Area to the Ducts}

As the piston acts as a valve for the inflow, transfer, and outflow ports by covering them at certain positions, this also affects the grid merging algorithm, described in Section 5.1 on page 65 . This is due to the feature that all ports connecting to the cylinder and crankcase can be realized as self contained meshes that are connected to the cylinder and crankcase by the grid merging method. It has been stated there that the connectivity information for each element $T_{j}$ on an inter-mesh boundary can be calculated at the initialization phase before the time iteration starts. Now, if $T_{j}$ is a cylinder or crankcase element that changes its size by the snapper algorithm this has to be accounted for in the grid merging structure. Thus, the area of a boundary element $T_{j}$ with all its neighbors $T_{j l k}\left(1 \leq k \leq k_{j l}\right)$ over the boundary face $S_{j l}$ has to be updated dynamically if its size was changed. But as there do not exist many boundary elements whose size is changed during a time step, and no new neighbors have to be found, this dynamic area update is not too computationally costly.

\subsubsection{The Algorithm}

In Flowchart 5.2.4 on the next page the complete algorithm of the piston motion is summarized. 
initialization

build layering structure of the cylinder and crankcase

time iteration

calculate CFL-condition

mesh movements

piston motion

calculate new piston position

\begin{tabular}{|l|l|l|}
\hline \multirow{2}{*}{ merge layer } & lowest layer too small \\
\hline split layer & lowest layer too large \\
\hline
\end{tabular}

move piston points

update data of resized elements

update window areas

crankshaft rotation

calculate fluxes

mesh adaption

time $=$ time $+\Delta t^{n}$

Figure 5.2.4: Flowchart of the moving piston algorithm. 


\subsection{The Crankshaft Motion}

The crankshaft, which rotates in the crankcase, possesses a complex three-dimensional structure. For the investigation of its influence on the flow behavior a threedimensional simulation is therefore indispensable.

We described different merging methods for a composite mesh in Section 5.1 on page 65. The same possibilities are given for the rotating mesh of the crankshaft within the outer mesh of the crankcase. Because of conservation issues we also decided here to use a patched grid approach. But due to the rotating motion of the crankshaft and the cylindrical shape of the mesh the straightforward implementation failed. The exhaustive search for new neighbors was too computationally expensive, and the approximation of the cylinder by planar cell faces produced too big errors because of the violation of the conservation property. Therefore, a new approach had to be found.

\subsubsection{Different Techniques}

Two approaches were tested. The fitted interface method and the curved interface method. Both are based on a cylindrical inner crankshaft mesh rotating in an outer crankcase mesh.

\section{The Fitted Interface Method}

If one tries to patch two grids together that do not fit exactly at the boundary (as on the left-hand side of Figure 5.3.1 on the next page) the conservation property is violated. This method overcomes this problems by splitting the faces of the elements in the outer crankcase mesh and fitting them exactly to the faces of the elements of the inner crankshaft mesh as shown in Figure 5.3.1 (right). Consequently, the conservation property is restored. Because of the rotation of the inner grid this is a dynamic process and the faces have to be updated every step.

In the following description it is assumed that the boundary of the two meshes is a piecewise linear approximation of a circle, shifted in $\mathrm{z}$-direction. Furthermore, the number and extension of the layers in $\mathrm{z}$-direction of the inner and outer mesh are expected to be identical. This enables us to reduce the problem to two dimensions, thus, we only need to calculate the first layer and can copy the result to the others.

Before each time step of the algorithm (refer to the complete algorithm in Paragraph 4.10 on page 63 ), the inner crankshaft mesh is rotated to correspond to the actual crankshaft angle. For this, each point is rotated from its initial position of $\theta_{0}:=\theta\left(t_{0}\right)=\theta(0)=0^{\circ}$ crankshaft angle to the new position $\theta_{\text {new }}:=\theta\left(t^{n+1}\right){ }^{2}$ Now this new position of the inner mesh is used to alter the boundary cells of the outer mesh. With the notation of Figure 5.3.1, face $S_{j l}$ of element $T_{j}$ is split into two facets (in this example). The point $\mathbf{p}_{1}$ is projected onto the face $S_{i k}$ of element $T_{i}$ along the extension of edge $E_{j k}$ of element $T_{j}$ :

$$
\mathbf{p}_{1, \text { new }}=\mathbf{p}_{1}-\frac{|\mathbf{u} \times \mathbf{w}|}{|\mathbf{u} \times \mathbf{d}|} \mathbf{d}
$$

\footnotetext{
${ }^{2}$ Whereas an incremental procedure, where each point is rotated from $\theta_{\text {old }}:=\theta\left(t^{n}\right)$ to $\theta_{\text {new }}$, would accumulate the errors of each change in position.
} 
with $\mathbf{u}=\mathbf{f}_{2}-\mathbf{f}_{1} \subset S_{i k}, \mathbf{w}=\mathbf{p}_{1}-\mathbf{f}_{1}$, and $\mathbf{d}=\mathbf{p}_{4}-\mathbf{p}_{1}$ forming edge $E_{j k}$. Now the volume of the facets $S_{j l 1}$, formed by the points $\mathbf{p}_{1}$ and $\mathbf{f}_{2}$, is calculated and stored in the grid merging structure together with the corresponding outer normal $\mathbf{n}_{i k}$ of face $S_{i k}$ of element $T_{i}$. Point $\mathbf{p}_{2}$ and the second facet $S_{j l 2}$ are treated similarly. After creating the two new entities in the grid merging structure, the other faces of the cell $T_{j}$ that have been changed in area by the movement of the points, as well as the cell volume, have to be updated. The shape of the frontal face might become quite irregular and has to be triangulated in order to calculate its area. This procedure is repeated for each boundary element in the first layer of the outer crankcase mesh and then the calculated information is copied to the other layers. Now the standard finite volume scheme extended by the facet handling, as described in Paragraph 5.1.4 on page 67 , can be applied.

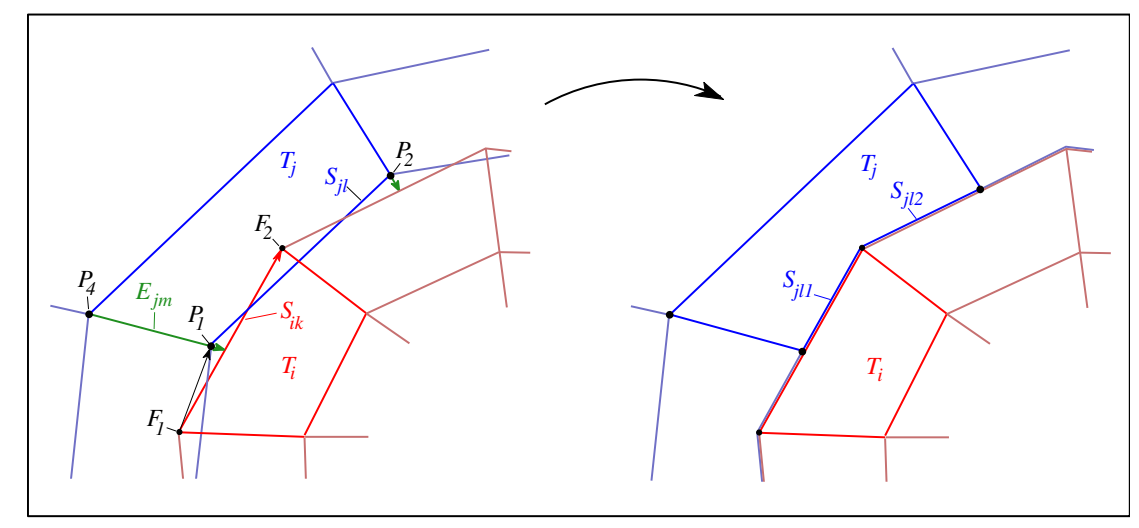

Figure 5.3.1: The fitted interface method fits the outer (blue) grid to the inner (red) one.

\section{The Curved Interface Method}

Because the fitted interface method needed a special layer at the outside of the inner crankshaft mesh (which needed to consist of very thin elements due to geometrical reasons ${ }^{3}$ ), and the dynamic splitting and moving of the element faces was still too time consuming, it was replaced by the curved interface method. This method treats the interface of the two meshes as a true cylinder $\mathcal{Z}:=S^{1} \times\left[z_{1}, z_{2}\right]$. Thus, the faces of the boundary elements are not planar any longer but curved in one direction. This also ensures the conservation property and a constant changing of face locations is overcome. As a further advantage the outer face of the crankshaft itself is simulated with its real round shape.

To avoid the thin outer layer of elements around the crankshaft, it is now admitted that the outer layer of the inner grid has holes, as shown in Figure 5.3.2 on the following page. With this method this does not pose a problem since no points need to be projected to the faces of the inner elements. In order to handle elements with curved faces some modifications to standard elements have to be considered. We

\footnotetext{
${ }_{3}^{3}$ There is not much space between crankshaft and crankcase wall.
} 
describe now the treatment for an element in the outer mesh with inwards curved face. The elements in the inner mesh with outwards curved faces are handled in an analogous manner.

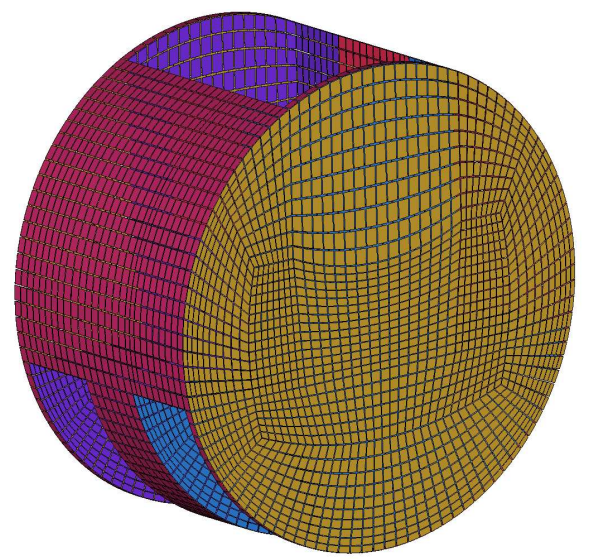

Figure 5.3.2: Holes are now possible in the outer layer of the crankshaft mesh.

Thus, all elements at the crankcase-crankshaft interface are adapted at initialization, as follows. The area of the curved face $S_{j l_{0}}$ increases whereas the area of the front and rear face, $S_{j l_{f}}$ and $S_{j l_{r}}$ respectively, and the volume $V_{T_{j}}$ decrease. In our case (cf. Figure 5.3.3 on page 82 ) these changes can be calculated easily:

$$
\begin{aligned}
\left|S_{j l_{0}}\right| & =\alpha_{0} R_{c s} l_{z} \\
\left|S_{j l_{f}, \text { new }}\right| & =\left|S_{j l_{f}, \text { old }}\right|-\left(\frac{\alpha_{0}}{2} R_{c s}^{2}-\frac{d}{2} R_{c s} \cos \left(\frac{\alpha_{0}}{2}\right)\right) \\
\left|S_{j l_{r}, \text { new }}\right| & =\left|S_{j l_{f}, \text { new }}\right| \\
V_{T_{j}} & =\left|S_{j l_{f}, \text { new }}\right| l_{z}
\end{aligned}
$$

where $\alpha_{0}:=\alpha_{P_{2}}-\alpha_{P_{1}}$, with $\alpha_{P_{1 / 2}}$ as in Figure 5.3.3 in degrees, $R_{c s}$ the radius of the crankshaft, $l_{z}$ the length of the cell in z-direction, and $d:=\left|\mathbf{p}_{2}-\mathbf{p}_{1}\right|=2 R_{c s} \sin \left(\frac{\alpha_{0}}{2}\right)$ the distance between points $P_{1}$ and $P_{2}$. The normal $\mathbf{n}_{j l_{0}}$ of face $S_{j l_{0}}$ is now not constant anymore but depends, in our case, i.e. the center of rotation is the origin and the axis of rotation is the $\mathrm{z}^{-}$-axis, on $\alpha(\mathbf{x}), \mathbf{x} \in S_{j l_{0}}$ :

$$
\mathbf{n}_{j l_{0}}(\alpha(\mathbf{x}))=\left(\begin{array}{c}
-\cos \alpha(\mathbf{x}) \\
-\sin \alpha(\mathbf{x}) \\
0
\end{array}\right)
$$

In the finite volume scheme given in Section 4.3 on page $47\left|S_{j l}\right| \mathbf{n}_{j l}$ is assumed to be an approximation of $\int_{S_{j l}} \mathbf{n}_{j l}(\cdot)$ which is not correct in the case of an evaluation of the normal $\mathbf{n}_{j l_{0}}(\mathbf{x})$ in a single point. But because of the explicit representation of the normal $\mathbf{n}_{j l_{0}}(\mathbf{x})$ and the rectangular shape of $S_{j l_{0}}$ an exact integration of $\mathbf{n}_{j l_{0}}(\mathbf{x})$ 
on $S_{j l_{0}}$ is possible:

$$
\int_{S_{j l_{0}}} \mathbf{n}_{j l_{0}}(\mathbf{x}) d \mathbf{x}=l_{z} R_{c s} \int_{\alpha_{P_{1}}}^{\alpha_{P_{2}}} \mathbf{n}_{j l_{0}}(\alpha) d \alpha=l_{z} R_{c s}\left(\begin{array}{c}
-\sin \alpha_{P_{2}}+\sin \alpha_{P_{1}} \\
\cos \alpha_{P_{2}}-\cos \alpha_{P_{1}} \\
0
\end{array}\right)
$$

Now, if in the finite volume scheme we replace $\mathbf{n}_{j l_{0}}$ by

$$
\overline{\mathbf{n}}_{j l_{0}}:=\frac{1}{\left|S_{j l}\right|} \int_{S_{j l}} \mathbf{n}_{j l_{0}}(\mathbf{x}) d \mathbf{x}=\frac{1}{\alpha_{0}}\left(\begin{array}{c}
-\sin \alpha_{P_{2}}+\sin \alpha_{P_{1}} \\
\cos \alpha_{P_{2}}-\cos \alpha_{P_{1}} \\
0
\end{array}\right)
$$

the standard algorithm can be applied to treat such elements with curved faces.

Lemma 5.3.1. For the curved face $S_{j l_{0}}$ let $\overline{\mathbf{n}}_{j l_{0}}$ be given by (5.3.1) and $\overline{\mathbf{n}}_{j l}=\mathbf{n}_{j l}$ for $l \neq l_{0}$. Then

$$
\sum_{l=1}^{k_{j}}\left|S_{j l}\right| \overline{\mathbf{n}}_{j l}=\mathbf{0}
$$

Proof. It is sufficient to show that $\left|S_{j l_{0}}\right| \overline{\mathbf{n}}_{j l_{0}}=\left|\tilde{S}_{j l_{0}}\right| \tilde{\mathbf{n}}_{j l_{0}}$ with $\tilde{S}_{j l_{0}}$ being the planar face and $\tilde{\mathbf{n}}_{j l_{0}}$ its constant outer normal vector. Then the proposition follows from the according property for elements with planar faces.

The extension in z-direction of $S_{j l_{0}}$ and $\tilde{S}_{j l_{0}}$ is identical. Therefore, we only have to show that

$$
l_{R} \overline{\mathbf{n}}_{j l_{0}}=d \tilde{\mathbf{n}}_{j l_{0}}
$$

with $l_{R}:=\frac{2 \pi}{360} \alpha_{0} R_{c s}$ the arc length from $P_{1}$ to $P_{2}$ and $d:=\left|\mathbf{p}_{2}-\mathbf{p}_{1}\right|=2 R_{c s} \sin \left(\frac{\alpha_{0}}{2}\right)$ the distance between $P_{1}$ and $P_{2}$. Equation (5.3.3) is true since

$$
R_{c s}\left(\begin{array}{c}
-\sin \alpha_{P_{2}}+\sin \alpha_{P_{1}} \\
\cos \alpha_{P_{2}}-\cos \alpha_{P_{1}} \\
0
\end{array}\right)=2 R_{c s} \sin \left(\frac{\alpha_{P_{2}}-\alpha_{P_{1}}}{2}\right)\left(\begin{array}{c}
-\cos \left(\frac{\alpha_{P_{2}}+\alpha_{P_{1}}}{2}\right) \\
-\sin \left(\frac{\alpha_{P_{2}}+\alpha_{P_{1}}}{2}\right) \\
0
\end{array}\right),
$$

which can be seen by applying the addition theorems for sine and cosine.

Remark 5.3.2. The property (5.3.2) is a necessary requirement for the convergence of the finite volume method. The following example illustrates this fact.

Assuming constant data $\mathbf{U}$ for element $T_{j}$ and all neighbors $T_{j l}\left(1 \leq l \leq k_{j}\right)$, one would expect the data to stay constant: $\mathbf{U}_{j}^{n+1}=\mathbf{U}_{J}^{n}$. However, in the finite volume scheme we obtain

$$
\begin{aligned}
\mathbf{U}_{j}^{n+1} & =\mathbf{U}_{j}^{n}-\frac{\Delta t^{n}}{\left|T_{j}\right|} \sum_{l=1}^{k_{j}} \mathbf{g}_{j l}(\mathbf{U}, \mathbf{U}) \\
& =\mathbf{U}_{j}^{n}-\frac{\Delta t^{n}}{\left|T_{j}\right|} \sum_{l=1}^{k_{j}}\left|S_{j l}\right| \mathbf{f}(\mathbf{U}) \cdot \mathbf{n}_{j l} \\
& =\mathbf{U}_{j}^{n}-\frac{\Delta t^{n}}{\left|T_{j}\right|} \mathbf{f}(\mathbf{U}) \cdot \sum_{l=1}^{k_{j}}\left|S_{j l}\right| \mathbf{n}_{j l},
\end{aligned}
$$


thus if $\mathbf{f}(\mathbf{U})$ does not vanish (i.e. pressure $p>0$ ) and equation (5.3.2) does not hold then $\mathbf{U}_{j}^{n+1} \neq \mathbf{U}_{J}^{n}$.

Now we have described the changes to the boundary elements to handle the curved surface at the initialization phase. On the inner mesh the orientation of the normals has to be updated after every movement of the cells during the time iteration but the sizes of the faces and the volume of the elements stays the same as well as all properties on the outer mesh. What we also have to consider is the possible changing of neighbors while the inner mesh rotates.

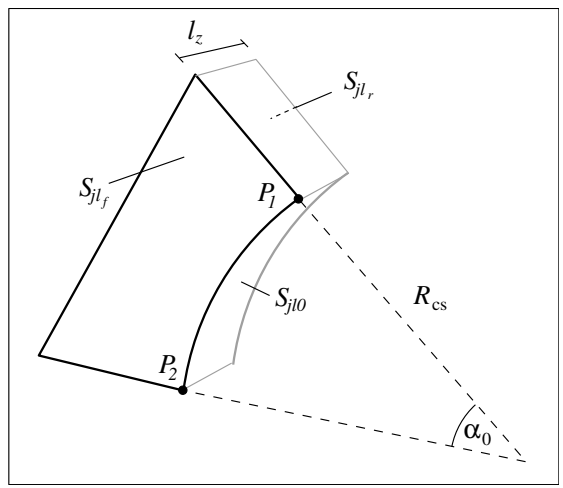

Figure 5.3.3: Round element faces in the crankcase and crankshaft meshes.

\subsubsection{Exploitation of the Quasi 2D Structure}

In order to speed up the neighbor search, a new structure was created that exploits the quasi 2D structure of the crankshaft-crankcase interface. A pointer to each element of the boundary is stored such that an element on the cylinder $\mathcal{Z}:=S^{1} \times\left[z_{1}, z_{2}\right]$ can be accessed by its position on the circle $S^{1}$ and its layer $n_{z}$. A search for an element is then performed by first looking for an element whose range on the $S^{1}$ is admissible, starting with the first element to the left of a former neighbor ${ }^{4}$. If this is found the z-range is checked for the elements in the correct layers. The worst-case complexity of this algorithm is $O(N)$ as opposed to $O\left(N^{2}\right)$ of the naive neighbor search. Because the inner mesh can have holes (the crankshaft itself) not every element in the outer mesh has a full set of neighbors. If a neighbor $T_{j l k}$ of element $T_{j}$ is found the area of the common facet $S_{j l k}$ has to be determined.

\footnotetext{
${ }^{4}$ The inner mesh rotates counter clockwise, therefore, the first element to the left has the highest probability of being the sought element.
} 
This is straightforwardly solved as

$$
\begin{aligned}
\left|S_{j l k}\right|= & l_{z}\left(T_{j}, T_{j l k}\right) l_{\alpha}\left(T_{j}, T_{j l k}\right), \\
l_{z}\left(T_{j}, T_{j l k}\right):= & \min \left\{\max _{i \in \mathcal{K}_{j}}\left\{\left(P_{i}\right)_{z}\right\}, \max _{i \in \mathcal{K}_{j l k}}\left\{\left(P_{i}\right)_{z}\right\}\right\} \\
& -\max \left\{\min _{i \in \mathcal{K}_{j}}\left\{\left(P_{i}\right)_{z}\right\}, \min _{i \in \mathcal{K}_{j l k}}\left\{\left(P_{i}\right)_{z}\right\}\right\}, \\
l_{\alpha}\left(T_{j}, T_{j l k}\right):= & \min \left\{\max _{i \in \mathcal{K}_{j}}\left\{\left(P_{i}\right)_{\alpha}\right\}, \max _{i \in \mathcal{K}_{j l k}}\left\{\left(P_{i}\right)_{\alpha}\right\}\right\} \\
& -\max \left\{\min _{i \in \mathcal{K}_{j}}\left\{\left(P_{i}\right)_{\alpha}\right\}, \min _{i \in \mathcal{K}_{j l k}}\left\{\left(P_{i}\right)_{\alpha}\right\}\right\},
\end{aligned}
$$

with $l_{z}$ being the interval of the overlap of $T_{j}$ and $T_{j l k}$ in z direction, and $l_{\alpha}$ the interval of the overlap on the circle $S^{1}$, where $\mathcal{K}_{j}$ is an index set such that $P_{i}\left(i \in \mathcal{K}_{i}\right)$ is a vertex of element $T_{j}$, and $\left(P_{i}\right)_{z}$ is the z coordinate of the point $P_{i}$, and $\left(P_{i}\right)_{\alpha}$ is its $\alpha$ coordinate with respect to the $S^{1}$ used above. Now, all entities of the grid merging structure are filled and the finite volume scheme can be calculated as usual.

\subsubsection{Compensation of the Artificial Transport}

Because of the rotation of the elements in the crankshaft mesh, also the data on these cells is rotated. However, this is unphysical as the rotation of a mesh without obstacles should leave the flow unchanged. Therefore, this artificial transport has to be compensated for. This is done by calculating a transport of all data in the opposite direction of rotation (motivated by the time-dependent formulation of the integral form of the Navier-Stokes equations in Paragraph 4.6 on page 54).

Thus, starting from the standard conservation law of transport

$$
\begin{gathered}
\int_{T_{j}} \frac{\partial}{\partial t} \mathbf{U}-\int_{\partial T_{j}} \mathbf{v}_{\text {grid }} \cdot \mathbf{n} \mathbf{U}=\mathbf{0}, \\
\mathbf{v}_{\text {grid }}=2 \pi \frac{\operatorname{rpm}}{60}\left(\begin{array}{c}
x_{2} \\
-x_{1} \\
0
\end{array}\right),
\end{gathered}
$$

with rpm being the rotations per minute of the crankshaft mesh, we get for the discrete explicit upwind finite volume scheme of first order (cf. Section 4.3 on page 47)

$$
\mathbf{U}_{j}^{n+1}=\mathbf{U}_{j}^{n}-\frac{\Delta t_{n}}{\left|T_{j}\right|} \sum_{l=1}^{k_{j}} \tilde{\mathbf{g}}_{j l}\left(\mathbf{U}_{j}^{n}, \mathbf{U}_{j l}^{n}\right)
$$

with

$$
\tilde{\mathbf{g}}_{j l}\left(\mathbf{U}_{j}^{n}, \mathbf{U}_{j l}^{n}\right)=\left\{\begin{array}{cl}
\left|S_{j l}\right| \mathbf{v}_{\text {grid }} \cdot \mathbf{n}_{j l} \mathbf{U}_{j l}^{n} & \text { if } \mathbf{v}_{\text {grid }} \cdot \mathbf{n}_{j l} \geq 0 \\
\left|S_{j l}\right| \mathbf{v}_{\text {grid }} \cdot \mathbf{n}_{j l} \mathbf{U}_{j}^{n} & \text { if } \mathbf{v}_{\text {grid }} \cdot \mathbf{n}_{j l}<0 \\
\mathbf{0} & \text { if } S_{j l} \text { is wall boundary }
\end{array} .\right.
$$


Therefore, we can write

$$
\mathbf{U}_{j}^{n+1}=\mathbf{U}_{j}^{n}-\sum_{l=1}^{k_{j}} \frac{\mathbf{v}_{\text {grid }} \cdot \mathbf{n}_{j l} \Delta t^{n}\left|S_{j l}\right|}{\left|T_{j}\right|}\left\{\begin{array}{cl}
\mathbf{U}_{j l}^{n} & \text { if } \mathbf{v}_{\text {grid }} \cdot \mathbf{n}_{j l} \geq 0 \\
\mathbf{U}_{j}^{n} & \text { if } \mathbf{v}_{\text {grid }} \cdot \mathbf{n}_{j l}<0 \\
\mathbf{0} & \text { if } S_{j l} \text { is wall boundary }
\end{array} .\right.
$$

Now, $V_{\text {swept }}:=\mathbf{v}_{\text {grid }} \cdot \mathbf{n}_{j l} \Delta t^{n}\left|S_{j l}\right|$ is the volume swept by the face $S_{j l}$ from time-step $n$ to time-step $n+1$ as shown in Figure 5.3.4 on the next page. This volume $V_{\text {swept }}$ can be calculated easily if one stores the coordinates of the mesh points from the last time-step. As we assumed a layered mesh, the front and back faces of the elements are parallel to the x-y axis, therefore, $\mathbf{v}_{\text {grid }} \cdot \mathbf{n}_{j l}=0$ for such faces $S_{j l}$. They do not account for any transport terms. Summarizing, our resulting compensation scheme, combined with the finite volume scheme for the discretization of the Navier-Stokes equations, can be calculated as

$$
\begin{aligned}
\mathbf{U}_{j}^{n+1}=\mathbf{U}_{j}^{n} & -\frac{\Delta t^{n}}{\left|T_{j}\right|}\left(\sum_{l=1}^{k_{j}} \mathbf{g}_{j l}\left(\mathbf{U}_{j}^{n}, \mathbf{U}_{j l}^{n}\right)-\sum_{l=1}^{k_{j}} \mathbf{G}_{j l}\left(\mathbf{U}_{j}^{n}, \mathbf{U}_{j l}^{n}\right)\right) \\
- & \sum_{\substack{l=1 \\
\mathbf{v}_{\text {grid }} \cdot \mathbf{n}_{j l} \neq 0}} \frac{V_{\text {swept }}}{\left|T_{j}\right|}\left\{\begin{array}{cl}
\mathbf{U}_{j l}^{n} & \text { if } \mathbf{v}_{\text {grid }} \cdot \mathbf{n}_{j l}>0 \\
\mathbf{U}_{j}^{n} & \text { if } \mathbf{v}_{\text {grid }} \cdot \mathbf{n}_{j l}<0 \\
\mathbf{0} & \text { if } S_{j l} \text { is wall boundary }
\end{array},\right.
\end{aligned}
$$

or equivalently

$$
\begin{gathered}
\overline{\mathbf{U}}_{j}^{n}=\mathbf{U}_{j}^{n}-\sum_{l=1}^{k_{\text {grid }} \cdot \mathbf{n}_{j l} \neq 0} \frac{V_{\text {swept }}}{\left|T_{j}\right|}\left\{\begin{array}{cl}
\mathbf{U}_{j l}^{n} & \text { if } \mathbf{v}_{\text {grid }} \cdot \mathbf{n}_{j l}>0 \\
\mathbf{U}_{j}^{n} & \text { if } \mathbf{v}_{\text {grid }} \cdot \mathbf{n}_{j l}<0 \\
\mathbf{0} & \text { if } S_{j l} \text { is wall boundary }
\end{array}\right. \\
\mathbf{U}_{j}^{n+1}=\overline{\mathbf{U}}_{j}^{n}-\frac{\Delta t^{n}}{\left|T_{j}\right|}\left(\sum_{l=1}^{k_{j}} \mathbf{g}_{j l}\left(\mathbf{U}_{j}^{n}, \mathbf{U}_{j l}^{n}\right)-\sum_{l=1}^{k_{j}} \mathbf{G}_{j l}\left(\mathbf{U}_{j}^{n}, \mathbf{U}_{j l}^{n}\right)\right),
\end{gathered}
$$

with $\mathbf{g}_{j l}$ and $\mathbf{G}_{j l}$ being the convective and viscous numerical fluxes of the NavierStokes discretization as defined in Sections 4.4 on page 50 and 4.5 on page 52. (For the treatment of moving grids compare also [FP97].)

\subsubsection{The Crankshaft-Transfer Port Interface}

Another problem that arises, is the interface between the rotating crankshaft mesh and the transfer port mesh. So far, we just discussed the search and area computation for the crankshaft-crankcase interface. As there is no special grid structure to profit from, the standard grid merging algorithm, as described in Section 5.1 on page 65 , is used. It is just extended by the ability to update the facet area of active neighbors, to look for new neighbors starting from the neighbor on the leading edge of the rotating mesh, and to respect the adapted area of the round faces. Especially the latter extension needs further consideration. As the frontal face $S_{j l_{f}}$ of 


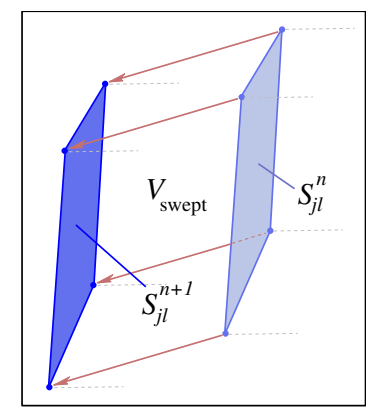

Figure 5.3.4: Swept volume of a face $S_{j l}$ at crankshaft rotation.

an element with rounded face $S_{j l_{0}}$ is bigger (for a cell in the inner mesh) or smaller (for a cell in the outer one), this has to be kept in mind at the calculation of the common area of the facet $S_{j l_{f} k}$ (see Lemma 5.3.1 on page 81 and Remark 5.3.2 on page 81). Thus, the arc segment of the frontal face $S_{j l_{f}}$ has to be calculated and intersected with the element faces of the neighbors $T_{j l_{f}}$ in the transfer port mesh. This calculation is of basic geometrical nature and is straightforward.

\subsubsection{The Algorithm}

In Flowchart 5.3.5 on the next page the complete rotating chrankshaft algorithm is summed up.

\subsection{The Connecting Rod}

The connecting rod is linked to the piston at one end and joined to the crankshaft at the other one. Therefore, its movement is a complicated tilting/shifting mechanism. It takes place in areas which interfere with the mesh rotation of the crankshaft and the piston motion. Thus, an easy treatment by blanking out cells or moving a whole mesh is not possible. In addition, the connecting rod is a rather small structure compared to the crankshaft and does probably not have an important impact on the flow pattern within the crankcase and ultimately does not change the scavenging process in the cylinder. In order to verify this hypotheses we conducted a series of two-dimensional numerical simulations of the crankcase with a moving connecting rod. The detailed descriptions of the employed model, the numerical methods and the meshes together with the achieved results are to be found in Chapter 8 on page 107. It was indeed revealed that even in the $2 \mathrm{D}$ case, where the connecting rod is a far more dominating structure than in $3 \mathrm{D}$, in the opening phase of the transfer ports, the flow pattern at the cylinder-transfer port interface hardly changes. The difference of the two flows, with and without connecting rod, was in every respect below $2 \%$. Thus it was concluded that the influence of the connecting rod can be neglected, even more so in 3D. A treatment of the movement of this structure is therefore, based on our data, not necessary. 
initialization

build curved interface structure of crankshaft-crankcase interface

build grid merging structure of crankshaft-transfer port interface

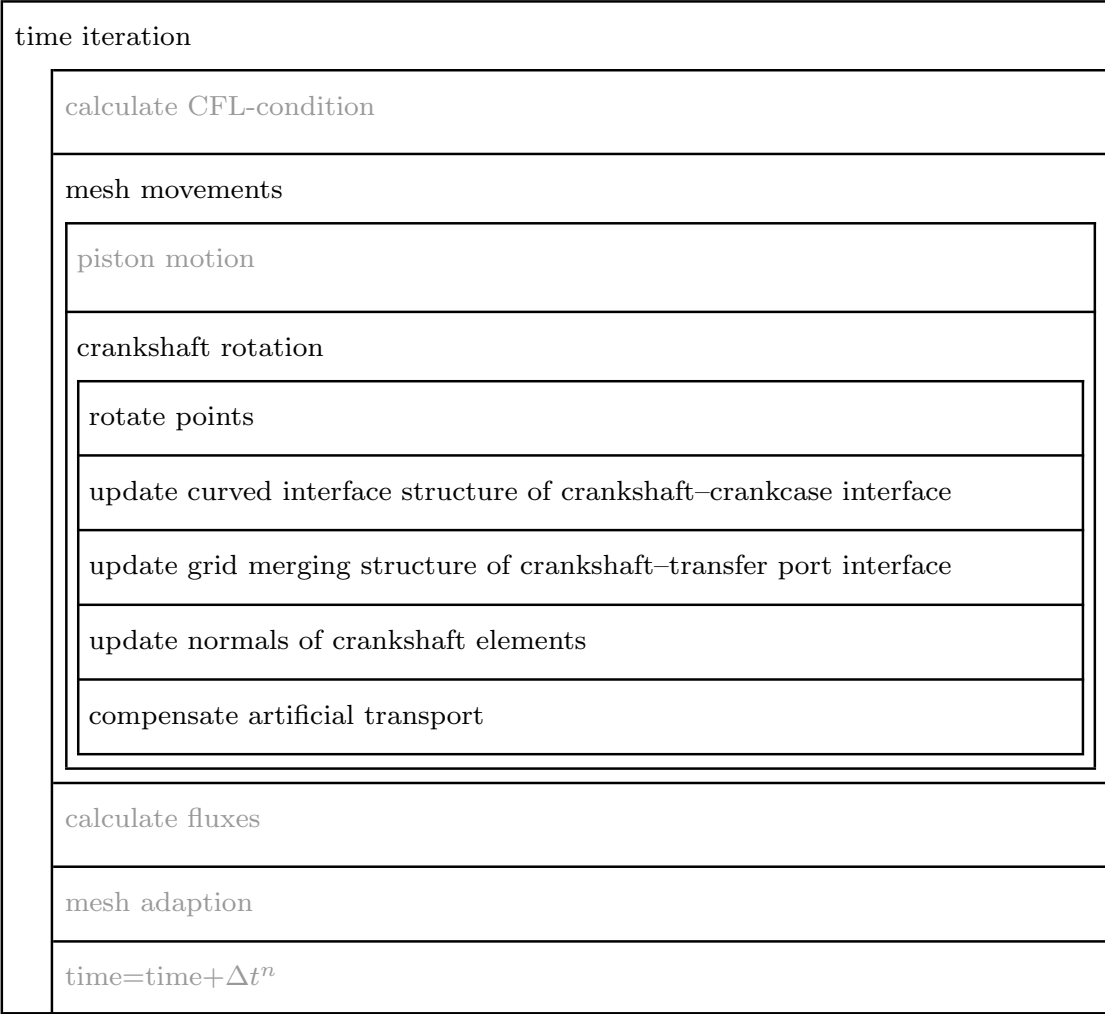

Figure 5.3.5: Flowchart of the rotating crankshaft algorithm. 


\section{Chapter 6}

\section{Reducing Computational Time}

In computational fluid dynamics computational time is always a topic. A mesh size that would be small enough to capture even the smallest feature of the flow field is still far beyond reach. In three dimensions, this problem is even intensified as the number of mesh cells rises with the order of $N^{3}$ with $N$ being the number of cells in one dimension. As one is also interested in an accurate solution with respect to the time scale, this also implies a restriction on the time step, which results in an increased number of time steps for a fixed time interval. This number also increases with the order $N$, resulting in a total complexity of the simulation of $O\left(N^{4}\right)$.

With the techniques described in the following, it is possible to reduce this cost in the temporal as well as in the spacial respect and keep the high accuracy of the fine mesh and small time-step.

\subsection{Temporally Consistent Adaptive Local Time-Step- ping}

Especially in dealing with complex geometries it is sometimes inevitable to have a mesh consisting of elements of largely differing size. The time-step $\Delta t^{n}$ from the finite volume scheme as given in Definition 4.3 .3 on page 49 depends, for stability reasons as stated in Section 4.8 on page 58, on the data $\mathbf{U}_{j}$ and element sizes of $T_{j}$. To assure the temporal consistency of the elements among themselves the smallest time-step $\Delta t^{n}:=\min _{j \in \mathcal{I}}\left\{\Delta t_{j}^{n}\right\}$ of all elements is chosen. Thus, small elements in the mesh force the time-step to be very small.

The idea of the adaptive local time-stepping is now to guarantee this temporal consistency by a different mechanism (see also [FBLV04, OMF+00]).

\subsubsection{The Time-Step Level}

Therefore, the time iteration is split up into time cycles of fixed duration. At the beginning of each cycle, at time $t^{n_{0}}$, the elements are sorted into a time level table according to their actual $\Delta t_{j}^{n_{0}}$ as follows. The element $T_{j}$ is in time level $l_{j}$ if its time-step $\Delta t_{j}^{n_{0}}$ is in the slice

$$
2^{l_{l}} \Delta t_{\min } \leq \Delta t_{j}^{n_{0}}<2^{l_{j}+1} \Delta t_{\min }
$$


with $0 \leq l_{j} \leq l_{\max }$ and $\Delta t_{\min }:=\min _{j \in \mathcal{T}}\left\{\Delta t_{j}^{n_{0}}\right\}$ (see Figure 6.1.1). Thus, at the beginning of the time cycle the values for $\Delta t_{\min }$ and $l_{\max }$ are calculated. Within the cycle, all elements belonging to time level $l_{\text {timestep }}=0$ are calculated each time-step with $\Delta t=\Delta t_{\min }$, the elements of level $l_{\text {timestep }}=1$ every second time-step with $2 \Delta t_{\text {min }}$, the elements of level $l_{\text {timestep }}=2$ every fourth one with $4 \Delta t_{\min }$, and so on. During this time stepping process the minimum time-step $\Delta t_{\text {min }}$ is fixed, but the classification of the $\Delta t_{j}^{n}$ of element $T_{j}$ to its time level $l_{j}$ is checked after each update of element $T_{j}$. I.e. if after the calculation of $T_{j}$ the new $\Delta t_{j}^{n+1}$ is found to have decreased below the bound $2^{l_{j}} \Delta t_{\min }$ its time level $l_{j}$ is reduced by one, and on the other hand if $\Delta t_{j}^{n+1} \geq 2^{l_{j}+1} \Delta t_{\min }$ the time level $l_{j}$ is increased by one. If the time level $l_{j}$ of element $T_{j}$ has to be decreased below level $l_{\text {timestep }}=0$ a new level $l_{\text {timestep }}=-1$ is created. Thus, the next time-step is conducted for the level $l_{\text {timestep }}=-1$ with $\Delta t=\frac{1}{2} \Delta t_{\min }$ and the restriction on $l_{j}$ has to be replaced by $l_{\min } \leq l_{j} \leq l_{\max }$. The time level $l_{j}$ can only be raised by one when the elements of time level $l_{\text {timestep }}=l_{j}+1$ have been updated in this step, because only then is element $T_{j}$ consistent with the other elements $T_{i}$ being in this higher time level.

By this method it is assured that every element is updated by the finite volume scheme with a $\Delta t$ that is smaller than or equal to the $\Delta t$ required by the CFLcondition. Furthermore, the temporal link between the elements is respected, since after the time cycle all elements advance to

$$
t^{n}=2^{l_{\max }} \Delta t_{\min }+t^{n_{0}}=\left(n-n_{0}\right) \Delta t_{\min }+t^{n_{0}},
$$

due to the duration of a complete time cycle of $2^{l_{\max }} \Delta t_{\min }$.

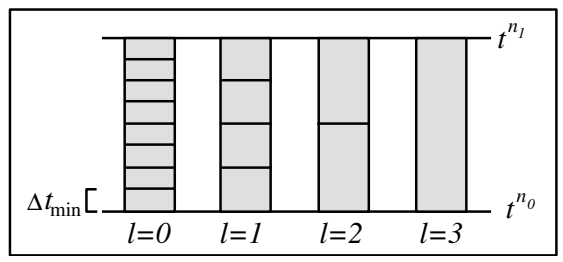

Figure 6.1.1: Time step levels.

Remark 6.1.1. It has to be noted that, in order to save computational time, the flux between two neighboring elements $T_{j}$ and $T_{j l}$ is only computed once, since it is required to be identical for conservation reasons. In addition to the speedup of the calculation, this is a crucial property for the local time stepping method to work. The reason being that after an update of the element $T_{j}$ with time level $l_{j}<l_{j l}$ the data $\mathbf{U}_{j}^{n-1}$ of time-step $n-1$ would be lost for the computation of the flux of element $T_{j l}$ with $T_{j}$. Thus a complete history of data $\mathbf{U}^{\tilde{n}}$ for $n_{0} \leq \tilde{n} \leq n$ would need to be stored, which is impossible in the case of large grids. Due to the one sided flux calculation, only data of not yet updated elements is needed which implies that only $\mathbf{U}^{n-1}$ and $\mathbf{U}^{n}$ have to be stored. 


\subsubsection{Forced Update of an Element}

It can occur that an element, or all elements, have to be updated. For instance, if an element has to be refined or coarsened (see the next Section 6.2 on page 91) or if the data on all elements is written into a file. Then, the element under consideration has to be calculated up to the actual time $t^{n}$. Therefore, it has to be determined when the element $T_{j}$ was last updated, which is basic calculus knowing the time level $l_{j}$ of the element, the minimum level $l_{\min }$, the starting point of the time cycle $n_{0}$, and the actual step $n$. This enables us to find the number of steps $n_{j}$ and the corresponding $\Delta t_{j}=n_{j} \Delta t_{\min }$ to update the element using the standard finite volume scheme. Now the data $\mathbf{U}_{j}$ of the element can be accessed. The new time level $l_{j}$ of the element is set to $l_{j}=l_{\min }$ and is adaptively raised during the next time-steps.

\subsubsection{Time Level Restrictions}

Not all elements can be in every time level. If the data of an element is changed by a mesh movement the element has to be updated at this moment. Therefore, if the geometry is adapted in each time level $l_{\text {geom }}$ to the actual situation, then this would also be the maximum level to be in for the elements affected by the geometry movement, i.e. all elements involved in the extended snapper algorithm of the piston (described in Section 5.2 on page 72) and all elements on the connecting crankcasecrankshaft interface. This restriction has to be verified each time an element tries to increase its time level due to a large enough $\Delta t_{j}^{n}$.

\subsubsection{Elements in the Rotating Crankshaft Mesh}

In order to not restrict the time level for all elements situated in the rotating crankshaft mesh, and therefore reducing the efficiency of the local time-stepping algorithm, a special treatment of the normals of these elements is necessary. As said in Section 5.3 on page 78, the normals of every element in the crankshaft mesh are updated according to the rotational position of the crankshaft. Thus, if an element is calculated only every $n_{j}^{\text {th }}$ time-step with corresponding time level $l_{j}>l_{\text {geom,crank }}$ ( $l_{\text {geom,crank }}$ being the update level of the crankshaft mesh rotation) it has to use average normals

$$
\overline{\mathbf{n}}_{j l}:=\frac{\mathbf{n}_{j l}^{n_{\text {old }}}+\mathbf{n}_{j l}^{n}}{2}
$$

with the normal $n_{\text {old }}$ of the last update of element $T_{j}$. Because of the one-sided update of the flux with the neighbors $T_{j l}$ of $T_{j}$, as noted in Paragraph 6.1.1 on page 87, this procedure does not violate the conservation property, and also Lemma 5.3.1 on page 81 is satisfied.

\subsubsection{The Algorithm}

The complete algorithm for the temporally consistent adaptive local time-stepping is presented in Flowchart 6.1.2 on the next page. 


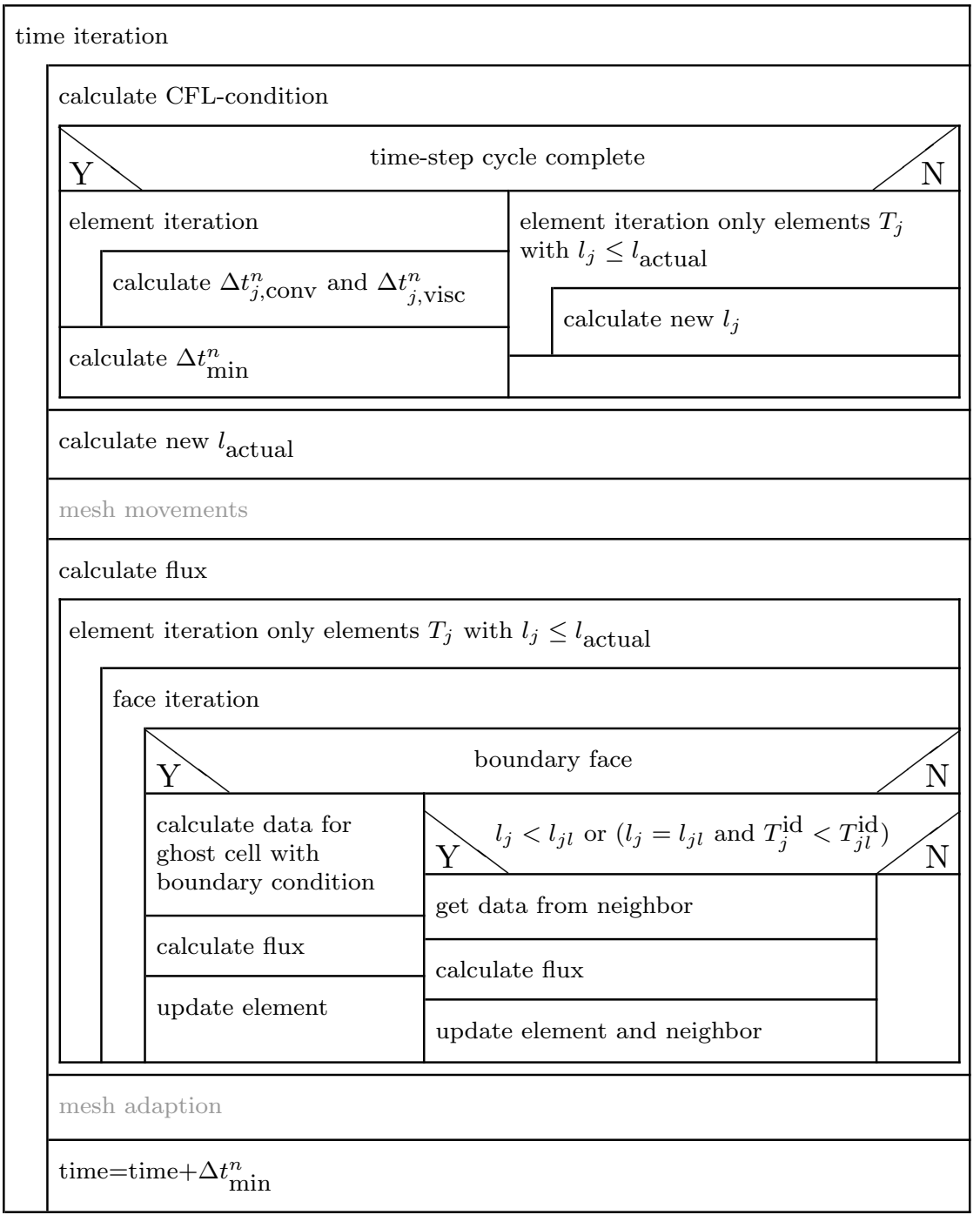

Figure 6.1.2: Flowchart of the local time-step algorithm. 


\subsection{Dynamic Local Mesh Adaption}

The idea behind the local mesh adaption is to approximate the solution of the considered problem with an equal distribution of the numerical error to reach a given accuracy with minimum computational cost. Thus, in areas where the approximation error is large the mesh should be refined, and where the error is small we can calculate on a coarse mesh to save resources. In the case of a solution evolving in time this adaption has to be dynamic.

Also, in the case of the dynamic mesh adaption it was necessary to extend the standard method for the handling of the moving meshes and the complex geometry.

\subsubsection{Different approaches}

In the literature, it is distinguished between adaption techniques that result in conformal meshes, i.e. meshes without hanging edges (cf. Definitions 4.2.2 on page 46 and 4.2.4 on page 47) and non-conformal meshes.

\section{Conformal Adaption}

One possibility is a re-meshing of the whole domain (as done in [HW92]) with a prescribed refinement in the areas indicated by the adaption criterion (see Paragraph 6.2.3 on the following page). This approach is ruled out for complex threedimensional hexahedral meshes, since an automatic mesh generation is not feasible in this case.

Another technique consists in moving the points of the discretization (cf. [VH93]) according to the adaption criterion, resulting in small elements where more accuracy is needed and large ones in areas of already good approximation. The number of elements stays constant in this approach.

The difficulty in these two approaches consists in the interpolation of the data $\mathbf{U}_{j}^{n}$ for the changed elements. As with the re-meshing and compression algorithms to realize the piston motion (cf. Paragraph 5.2.1 on page 72) this can be very complex and time consuming.

\section{Non-Conformal Adaption}

Therefore, a local adaption procedure is used. A local refinement of a hexahedral mesh always implies the presence of nonconforming (hanging) edges, as opposed to a tetrahedral one (see [Bän89, Bän91]). If indicated by the adaption criterion an element of the mesh is refined by splitting it up into several smaller child elements. For coarsening an element this process is reversed. Thus, a local interpolation of the data is possible (see Paragraph 6.2.2 on the following page).

\subsubsection{The Mesh Structure}

A structure has to be devised which is able to handle the difficulty of nonconformal meshes. Therefore, the local mesh adaption was realized with an underlying hierarchical mesh structure (compare [Gra99, NORS97, OR97, WR92]). The macro grid consists of the initially generated mesh. Each macro element can now 
be refined into child elements, which themselves can be further refined. Also, a coarsening is possible which is necessary for time-dependent problems.

\section{Geometrical procedure}

On refining, in our case, a hexahedron, it is divided into eight child hexahedrons, where the new vertices of the children are generated by the arithmetic mean of the vertices of the parent hexahedron, i.e. the new vertex $P_{12}$ on the edges between vertices $P_{1}$ and $P_{2}$ of the parent element is given by $\mathbf{p}_{12}=\frac{\mathbf{p}_{1}+\mathbf{p}_{2}}{2}$, the new vertex on the face by $\mathbf{p}_{1234}=\frac{1}{4} \sum_{i=1}^{4} \mathbf{p}_{i}$, and the volume vertex by $\mathbf{p}_{1 \ldots 8}=\frac{1}{8} \sum_{i=1}^{8} \mathbf{p}_{i}$. Each element is situated in a refinement level $l^{\text {ref }}$ depending on the depth in the hierarchical mesh. Macro elements are in refinement level $l^{\text {ref }}=0$, their children in level $l^{\text {ref }}=1$, and so forth. Elements of level $l^{\text {ref }}>0$ can be coarsened again if they do not have any child elements. The refinement level difference of neighboring cells is restricted to one. Therefore, in the case of hexahedrons the maximum number of neighbors $T_{j l k}$ of element $T_{j}$ per side $S_{j l}$ is four: $k \in\{1, \ldots, 4\}$. This is the case if the neighboring element is on refinement level $l_{j l}=l_{j}+1$. That implies that if an element has to be refined all its neighbor elements have to be on the same or a higher level: $l_{j l} \geq l_{j}$ for $1 \leq l \leq k_{j}, k_{j}$ being the number of faces of element $T_{j}$. If this precondition is not fulfilled then all neighboring elements with lower level have to be refined themselves. This has to be recursively iterated until an admissible state is reached. Inversely an element can only be coarsened if none of its neighbors has a higher refinement level: $l_{j l} \leq l_{j}\left(1 \leq l \leq k_{j}\right)$.

\section{Prolongation and Restriction of the Data}

At refinement the data $\mathbf{U}_{j}^{\text {parent }}$ of a parent element is just constantly prolonged to the child elements

$$
\mathbf{U}_{j}^{\text {child } i}:=\mathbf{U}_{j}^{\text {parent }} \quad(1 \leq i \leq 8) .
$$

This is obviously conservative since $\left|T_{j}\right|=\sum_{i=1}^{8}\left|T_{j}^{\text {child } i}\right|$.

When coarsening an element the data of the parent is obtained by a volume weighted averaging of the child data, i.e.

$$
\mathbf{U}_{j}^{\text {parent }}:=\frac{1}{\left|T_{j}\right|} \sum_{i=1}^{8}\left|T_{j}^{\text {child } i}\right| \mathbf{U}_{j}^{\text {child } i} .
$$

Also, with this procedure the conservation property holds, since

$$
\int_{T_{j}} \mathbf{U}_{j}^{\text {parent }}=\left|T_{j}\right| \mathbf{U}_{j}^{\text {parent }}=\sum_{i=1}^{8}\left|T_{j}^{\text {child } i}\right| \mathbf{U}_{j}^{\text {child } i}=\sum_{i=1}^{8} \int_{T_{j}^{\text {child } i}} \mathbf{U}_{j}^{\text {child } i} .
$$

\subsubsection{Mesh Adaption Criteria}

Now we have to decide which elements of the grid are to be refined and where a coarsened mesh is sufficient. Therefore, we would need to know the numerical error on each cell at each time-step (cf. [Kal96b]). 


\section{Error Estimator}

For linear elliptic or parabolic partial differential equations rigorous a-posteriori error estimates of the form

$$
\left\|u-u_{h}\right\| \leq C h^{\alpha} \eta\left(u_{h}\right)
$$

with an adequate norm $\|\cdot\|$, the exact and approximated solution $u$ and $u_{h}$ respectively, a constant $C, \alpha$ positive and a term $\eta\left(u_{h}\right)$ depending on the problem, are well-established. This implies that the numerical error can be reduced by decreasing the mesh size.

For the considered complex flow problem, i.e. the time-dependent convection dominant Navier-Stokes equations on a bounded domain in three space dimensions, no such a-posteriori error estimations are available up to now. Although for a model problem

$$
\left\{\begin{aligned}
u_{t}+\nabla \cdot f(u)-\varepsilon \triangle u & =0 & & \text { in } \mathbb{R}^{2} \times\left(0, T_{\text {end }}\right) \\
u(x, y, 0) & =u_{0}(x, y) & & \text { in } \mathbb{R}^{2}
\end{aligned}\right.
$$

with $\varepsilon \geq 0$ as a diffusion parameter, a-posteriori error estimates have been established (see [KO00, Ohl01]). Furthermore, in [OV03] an a-posteriori error estimate has been given for the boundary value problem in a multi dimensional setting for the scalar case for a general conservation law. Thus, this is a research field of special interest.

\section{Error Indicator}

Several approaches can be exploited to derive an error indicator. First of all, the residual of the numerical approximation gives a first hint of the location of the error (see e.g. [Wie95]). For finite difference schemes for a conservation law in one space dimension a rigorous error estimator has been derived by TADMOR et al. in [NTT94]. However, the used norms cannot be computationally evaluated.

Another method is to calculate the approximate solution on two meshes, one of them having a smaller mesh size. Then, in areas of large difference between the two solutions a further refinement would be indicated.

A similar technique uses the difference of a numerical solution of higher order to one of first order (as done e.g. in [Muz94]). Also, here a big difference is a sign for mesh refinement. As the latter two ideas are very time consuming and complex, heuristic criteria are used most often.

\section{Heuristic Criteria}

Heuristic indicators are usually based on local gradients. A large part of the numerical error occurs normally at discontinuities, i.e. the shocks and contact discontinuities, which are badly resolved on a discrete mesh and induce numerical diffusion. These structures can be detected by the use of gradients of key variables. This procedure is founded on theoretical results ([Cha98, Oh101]) and numerous numerical experiments (see e.g. [Geß94, Geß01, KKO02, GK01a, GK01b]). 


\subsubsection{The Marking Strategy}

For marking an element to be refined or coarsened, we use a heuristic strategy which is easy and fast to compute as it employs only readily available quantities (compare the previous Paragraph 6.2.3 on page 92 and [Geß01]).

Definition 6.2.1. (Local weighted flow quantity) Let $u_{j}^{n}:=\left(\mathbf{U}_{j}^{n}\right)_{k}, k \in\{1, \ldots, 5\}$ being a scalar component of the vector of conservative variables of element $T_{j}$ at time $t^{n}$ and $\mathbf{w}_{j}$ being the center of gravity of $T_{j}$. Then (for unrefined neighbors)

$$
\eta_{j}^{n}:=\left(\left|T_{j}\right|\left(\left(\frac{u_{j}^{n}-u_{j}^{n-1}}{\Delta t^{n}}\right)^{2}+\sum_{l=1}^{k_{j}}\left(\frac{u_{j}^{n}-u_{j l}^{n}}{\left|\mathbf{w}_{j}-\mathbf{w}_{j l}\right|}\right)^{2}\right)\right)^{\frac{1}{2}}
$$

defines the local weighted flow quantity. In the case of a refined neighbor $T_{j l}$ the following definition

$$
\eta_{j}^{n}:=\left(\left|T_{j}\right|\left(\left(\frac{u_{j}^{n}-u_{j}^{n-1}}{\Delta t^{n}}\right)^{2}+\sum_{l=1}^{k_{j}}\left(\frac{1}{k_{j l}} \sum_{k=0}^{k_{j l}} \frac{u_{j}^{n}-u_{j l k}^{n}}{\left|\mathbf{w}_{j}-\mathbf{w}_{j l k}\right|}\right)^{2}\right)\right)^{\frac{1}{2}}
$$

with $k_{j l}$ the number of facing Elements $T_{j l k}$ on face $S_{j l}$ with values $u_{j l k}^{n}$ and center of gravity $\mathbf{w}_{j l k}$ is used.

The mesh adaption criterion is then the relative value, which corresponds to an evenly distributed approximation error.

Definition 6.2.2. (Local relative mesh adaption quantity) Let

$$
\bar{\eta}^{n}:=\frac{1}{\sharp \mathcal{T}} \sum_{\substack{j \\ T_{j} \in \mathcal{T}}} \eta_{j}^{n}
$$

be the average of $\eta_{j}^{n}$ on the mesh $\mathcal{T}$, with $\sharp \mathcal{T}$ being the number of elements in $\mathcal{T}$. Then

$$
\zeta_{j}^{n}:=\frac{\eta_{j}^{n}}{\bar{\eta}^{n}}
$$

is the local relative mesh adaption quantity on element $T_{j}$ at time $t^{n}$.

Now the mesh adaption criterion, when to refine or to coarsen an element, can be stated.

Definition 6.2.3. (Local mesh adaption criterion) Let $\zeta_{j}^{n}$ be the local relative mesh adaption quantity of element $T_{j}$ at time $t^{n}$ from Definition 6.2.2. Then the element $T_{j}$ is marked for refinement or coarsening according to:

$$
\begin{array}{ll}
T_{j} \text { marked for refinement } & \text { if } \zeta_{j}^{n}>C_{\text {refine }} \\
T_{j} \text { marked for coarsening } & \text { if } \zeta_{j}^{n}<C_{\text {corsen }}
\end{array}
$$

with positive constants $C_{\text {refine }}>C_{\text {coarsen }}$. 
Remark 6.2.4. (Choice of $C_{\text {refine }} / C_{\text {coarsen }}$ ) As described in [Geß01], the constants to control the mesh adaption $C_{\text {refine }} / C_{\text {coarsen }}$ are chosen depending on the desired adaption accuracy. They can lie between $C_{\text {refine }} / C_{\text {coarsen }}=0.5 / 0.1$ for a very sensitive mesh refinement up to $C_{\text {refine }} / C_{\text {coarsen }}=1.3 / 0.7$ if the mesh should only be refined at intense structures in the numerical solution.

Remark 6.2.5. (Used components for marking) As indicators for the marking strategy we use the density $\rho$, the fresh-gas concentration $\sigma$, and the pressure $p$.

\subsubsection{Special Mesh Considerations}

This standard algorithm now needs further extensions in order to work with the above described mesh treatment for complex geometries with moving parts.

\section{Boundary Fitting}

As the boundary of our domain is not planar but curved in most parts, the new boundary vertices of refined elements do not lie exactly on the boundary. These vertices can be projected onto the exact geometrical boundary if the surface of this boundary is known. Thus, for a complete treatment of all boundaries it would be necessary to use the CAD data of the geometry. Since this data is a very complex structure, and as an initial mesh of fine enough resolution approximates even complex geometries quite well, such a projection is only needed in critical areas. Especially at the inter-mesh interfaces where the ports connect to the cylinder and crankcase, and at the crankshaft-crankcase interface, a high accuracy is desired. But the exact geometry of the lower part of the cylinder and the upper part of the crankcase is also known without extraction from the CAD data. They are just cylindrical bodies with given radius. Therefore, a projection onto these surfaces is a straightforward trigonometrical calculation.

\section{Moving Mesh Interaction}

Elements in the rotating crankshaft mesh are constantly changed. Also, the newly created vertices of refined elements have to be included in the rotational movement and the update of their outer normals $\mathbf{n}_{j l}$. An adaptive treatment of the crankshaft-crankcase interface has been avoided by initially refining this part of the mesh up to the maximum refinement level and fixing it for the time iteration. This has been done for efficiency reasons. It is too time consuming to update the concerned structures with every refinement. Also, a high accuracy is needed on this interface, thus justifying the computation on the maximum refinement level.

At the moving boundary just above the piston crown, and similarly on the uppermost layer below the piston in the crankcase, the mesh is likewise refined to maximum depth. The reason also being time considerations within the moving piston algorithm and the desired accuracy in this region of high activity. But unlike in the case of the rotating crankshaft mesh, this refinement needs constant updating due to the snapper algorithm described in Section 5.2 on page 72. If, during the upwards motion of the piston, a merging of two layers above the piston occurs then 
a further refinement of the cells in the second layer above the piston might be necessary (see the left-hand side of Figure 6.2.1). Inversely, at the downward motion a refinement, induced by the piston motion, is not needed anymore after the splitting of two layers. Therefore, a coarsening can be conducted if the local mesh adaption criterion indicates this (as done in Figure 6.2.1 (right)). Furthermore, special care has to be taken at the neighbors of moved elements in the crankcase mesh below the piston (see Figure 6.2.2) whose data is updated during the snapper algorithm as described in Paragraph 5.2.3 on page 74. These elements have to be refined to maximum level as well in order to treat them with the procedure described in Paragraph 5.2.3 on page 74 .

Remark 6.2.6. (Modified marking strategy) Due to the strong influence of the mesh movement on the temporal part of the marking strategy, this part is omitted in the two-stroke engine simulation.
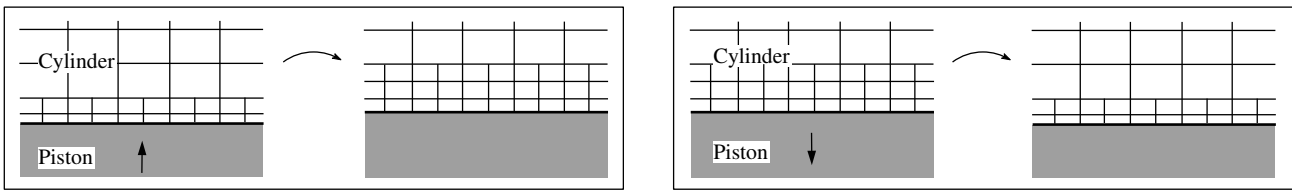

Figure 6.2.1: Refinement (left) and coarsening (right) induced by piston motion.
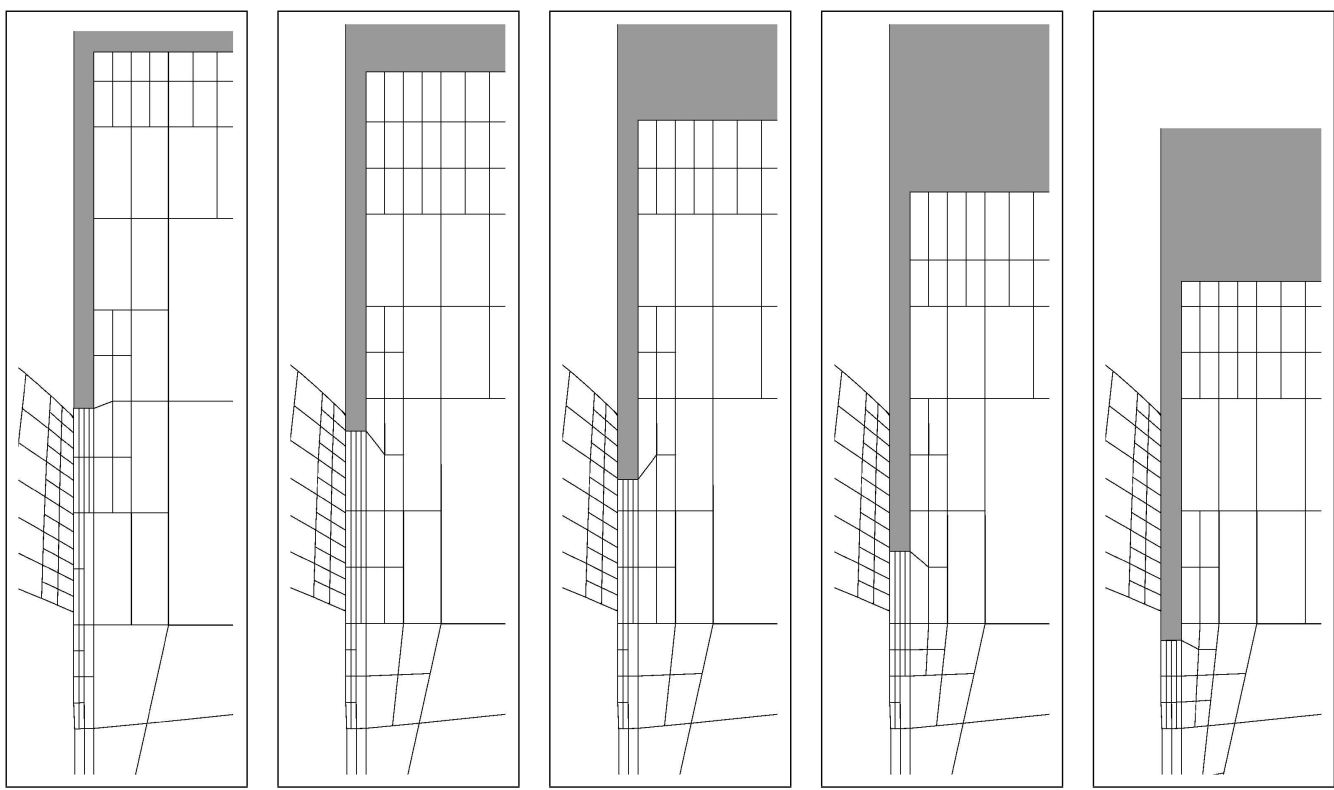

Figure 6.2.2: Neighbors which are influenced by the piston motion have to be refined $\left(0^{\circ}-80^{\circ}\right.$ crankshaft angle). 


\section{Merged Meshes}

For the purpose of achieving a high accuracy on the inter-mesh interfaces, and to avoid an excessive calculation of the area of common facets of neighboring elements across the contact boundary, we also decided in this case to refine these boundaries up to the maximum refinement level. The update of the merging grid structure therefore only has to be done at initialization time and not dynamically during the time stepping iteration.

\subsubsection{The Algorithm}

The complete algorithm of the dynamic local mesh adaption is summarized in Flowchart 6.2 .3 on the following page.

\subsection{Accelerated Convergence by Enhanced Initial Data}

In our application of simulating the two-stroke engine, we are in the situation of a periodic problem. After a full rotation of the crankshaft, i.e. $360^{\circ}$ crankshaft angle, the mesh, including all boundary conditions ${ }^{1}$, is in an identical state. Therefore, one would expect the flow pattern, i.e. the approximate solution $\mathbf{U}_{h}$, also to be periodic with period length of $360^{\circ}$ crankshaft angle. For this, the disturbing influence of the initial data has to diminish. This weakening of the influence needs many rotations of the crankshaft to take place. To avoid the lengthy calculations of the flow on a fine mesh for many periods we use an iterative scheme of constantly decreasing mesh size $h$.

\subsubsection{About the Notion of Convergence in Time for Periodic Prob- lems}

A convergence in time is usually defined as

$$
u_{h}(t, \cdot) \longrightarrow \bar{u}_{h}(\cdot) \quad \text { for } t \rightarrow \infty
$$

or, equivalently,

$$
\forall \varepsilon \exists T_{1}:\left\|u_{h}(t, \cdot)-\bar{u}_{h}(\cdot)\right\|<\varepsilon \text { for } t>T_{1}
$$

for a suitable norm $\|\cdot\|$. However, for a periodic time-dependent solution $u_{h}$ this is not a meaningful definition. It has to be adapted to the periodic situation:

$$
\forall \varepsilon \exists T_{1}:\left\|u_{h}(t+\tau, \cdot)-u_{h}(t, \cdot)\right\|<\varepsilon \text { for } t>T_{1}
$$

with $\tau$ being the period length, i.e. the solution $u_{h}$ does not change much from one period to the next one. Whereas within one period the solution is allowed to change.

\footnotetext{
${ }^{1}$ Except possibly the outflow boundary condition, which depends also on the flow itself.
} 


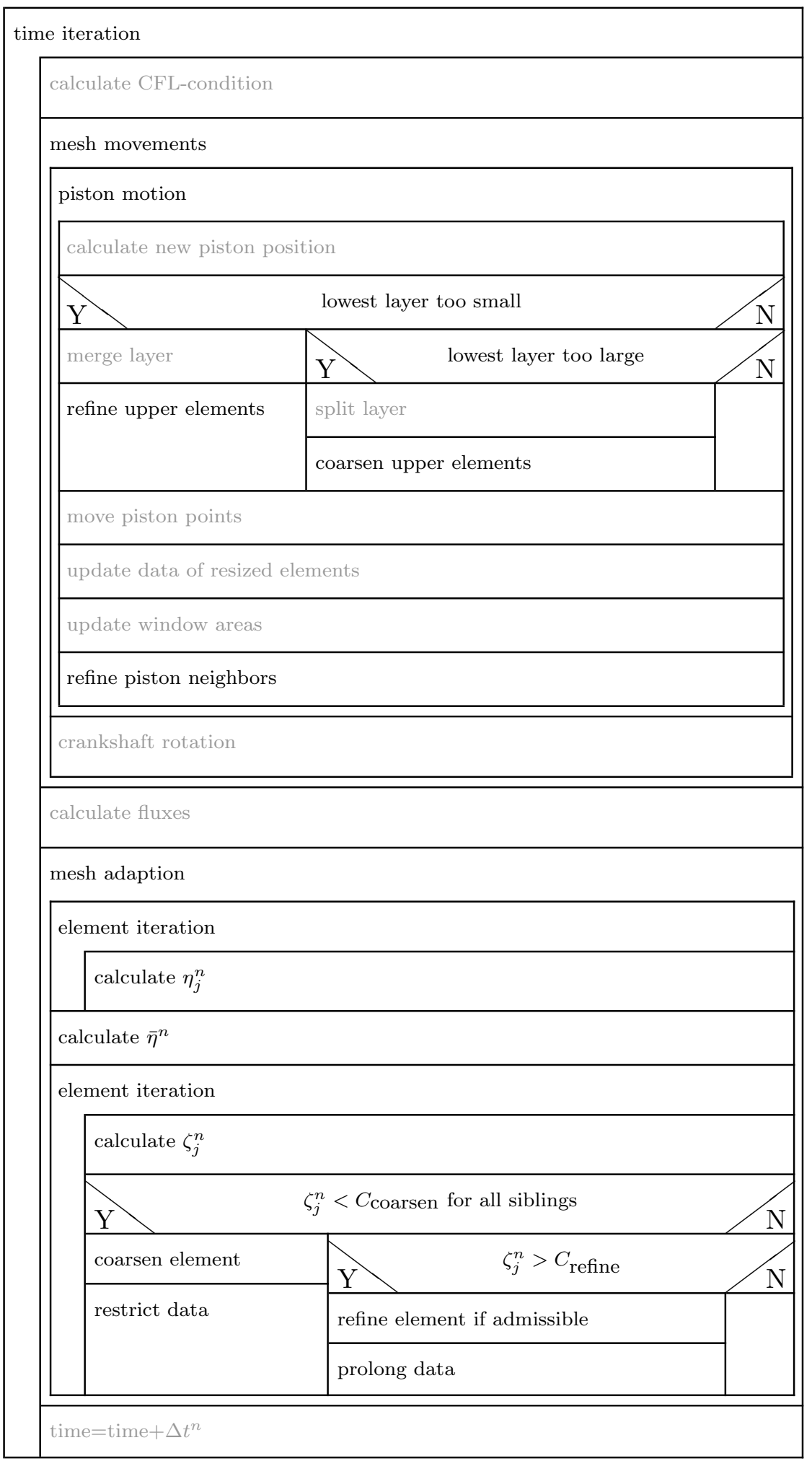

Figure 6.2.3: Flowchart of the mesh adaption algorithm. 


\subsubsection{Criteria for the Convergence in Time}

Using criteria (6.3.1) we have now to think about a suitable norm $\|\cdot\|$ and a threshold $\bar{\varepsilon}$ to stop the calculation. There are many possibilities to choose from:

- The most accurate would be to take into account the full solution $\mathbf{U}_{h}$ on every element $T_{j} \in \mathcal{T}$ on all time-steps $t^{n}$ ( $n$ such that $\left.T_{1} \leq t^{n}<T_{1}+\tau\right)$. Thus, e.g. for the discrete $L^{1}$-Norm at time $t_{0}$ the error $\varepsilon_{1}\left(t_{0}\right)$ would be:

$$
\varepsilon_{1}\left(t_{0}\right):=\sum_{n=n_{0}-m+1}^{n_{0}} \sum_{T_{j} \in \mathcal{T}}\left|\mathbf{U}_{j}^{n}-\mathbf{U}_{j}^{n-m}\right|
$$

with $n_{0}$ such that $t^{n_{0}}=t_{0}$ and $m$ such that $t^{n_{0}-m}=t_{0}-\tau$. As this would imply storing $\mathbf{U}_{h}$ for all time-steps over a full period length $\tau$ this approach is ruled out.

- To avoid this need for excessive storage one could compare two solutions $u_{h}$ on every element $T_{j}$ only at fixed time intervals, saying always at $\theta_{0}=0^{\circ}$ crankshaft angle:

$$
\varepsilon_{2}\left(t_{0}\right):=\sum_{T_{j} \in \mathcal{T}}\left|\mathbf{U}_{j}^{n_{0}}-\mathbf{U}_{j}^{n_{0}-m}\right|
$$

with $n_{0}$ and $m$ as above. The drawback of this method is that the information of the events during the period is not taken into account.

- The way out of this problem is to consider not the whole solution $\mathbf{U}_{h}$ on every element $T_{j}$ but to define characteristical values to describe the solution at a certain point in time. As described in Section 2.3 on page 11, there are some important characteristics for the description of the scavenging process of the engine, which we are interested in. Namely the delivery ratio $D R$, the scavenge ratio $S R$, the scavenging efficiency $S E$, and the trapping efficiency $T E$. Additionally, one could use the difference of the mass supplied and the mass outflow during one period:

$$
\varepsilon_{3}\left(t_{0}\right):=\sum_{n=n_{0}-m+1}^{n_{0}}\left(-\sum_{S_{j l} \in \mathcal{I}}\left|S_{j l}\right| \rho_{j} \mathbf{v}_{j} \cdot \mathbf{n}_{j l}-\sum_{S_{j l} \in \mathcal{O}}\left|S_{j l}\right| \rho_{j} \mathbf{v}_{j} \cdot \mathbf{n}_{j l}\right)
$$

with index-sets $\mathcal{I}$ and $\mathcal{O}$ for the faces $S_{j l}$ situated at the inflow and outflow boundaries respectively. A convergent state is therefore assumed if the massfluxes are nearly balanced over one period, i.e. $\varepsilon_{3}$ is smaller than a given threshold $\bar{\varepsilon}$.

\subsubsection{The Mesh Iteration}

To avoid the simulation on the final mesh until convergence in time is reached, which could very well imply calculating some one hundred periods of crankshaft rotation, depending on the threshold $\bar{\varepsilon}$, we conduct these calculations on a very coarse grid. The converged solution of this coarse grid is then taken as initial data 
for the next finer grid. This initial data is obtained by interpolation of the data on the coarse grid. This process is iterated until the final mesh is reached. There, only some three to four rotations of crankshaft are required to fulfill the same convergence criterion with threshold $\bar{\varepsilon}$. The exact value of the threshold $\bar{\varepsilon}$ is given in Chapter 10 on page 167, besides further details concerning the meshes and other configuration particulars.

\subsubsection{Mesh Dependency Checking}

By simulating the flow on meshes with varying mesh size, a check for the mesh dependency of the final solution is possible. Thus, we can inspect the convergence of $u_{h}$ for decreasing mesh size $h$ :

$$
u_{h} \longrightarrow \bar{u} \quad \text { for } h \rightarrow 0
$$

for an appropriate norm. In our case, this is again not done with the whole solution $\mathbf{U}_{j}^{n}$ ( $j$ such that $T_{j} \in \mathcal{T}$, and $n$ such that $\left.T_{\text {end }}-\tau<t^{n} \leq T_{\text {end }}\right)$ but by comparing on the different meshes the most important characteristical properties, scavenge ratio $S R$ and trapping efficiency $T E$, of the final crankshaft rotation period. The results of these convergence investigations are also described in Chapter 10 on page 167. 


\section{Chapter 7}

\section{Parallelization}

"Most applications have an unbounded appetite for memory and processor time. The efficient use of computing resources is, therefore, always a concern in scientific computing."

E.F. VAN DE VELDE [vdV94]

This citation emphasizes the desire of every CFD simulation to be as accurate as possible and to calculate problems with ever-increasing complexity within a reasonable time. By the use of parallel computers, the runtime of a problem can be reduced considerably. Also, the available memory in parallel computers is many times bigger.

\subsection{Efficiency of a Parallel Algorithm}

The reason for parallel computing is founded in the need to execute an identical problem in less time (speed-up) or a larger problem in the same time (scalability). The following definitions are used to measure these quantities for a given algorithm.

Definition 7.1.1. (Speed-up) The speed-up $S p(n)$ is defined as the ratio of the sequential total execution time $T(1)$ to the time needed with $n$ processors $T(n)$ :

$$
S p(n):=\frac{T(1)}{T(n)}
$$

Definition 7.1.2. (Efficiency) The efficiency $E$ describes the ratio of actual speedup $S p(n)$ to ideal speed-up $S p_{\text {opt }}(n)=n$ (see Remark 7.1.4 on the following page):

$$
E(n):=\frac{S p(n)}{n}
$$

Definition 7.1.3. (Scalability) Let an $n$-scaled problem $n P$ for a problem $P$ be given by $T_{n P}(1)=n T_{P}(1)$, where $T_{P}(1)$ is the serial execution time for $P$. Then the scalability $S c(n)$ is defined by:

$$
S c(n):=\frac{T_{P}(1)}{T_{n P}(n)}
$$


Remark 7.1.4. The theorem on the limitation of speed-up states that $S p(n) \leq n$ (see, e.g. [Sch99]) ${ }^{1}$. Therefore, it follows for the efficiency and scalability $E(n) \leq 1$ and $S c(n) \leq 1$.

The speed-up and scalability of an algorithm are determined by the portion of the work load $T_{\text {par }}$ that can be distributed between the processors and the work load $T_{\text {ser }}$ that needs to be executed by only one processor. An ideal speed-up $S p(n)=n$ is obtained if $T_{\text {ser }}=0$, i.e. the algorithm is fully parallelized and the whole load can be distributed between the $n$ processors (see also Remark 7.1.6). This is, e.g., the case in Monte-Carlo simulations, where the single tasks are completely independent of each other. But, in general, $T_{\text {ser }} \neq 0$, thus it follows for the speed-up $S p(n)=T_{\text {ser }}+\frac{T_{\text {par }}}{n}$. This is, if $T_{\text {ser }}$ and $n$ are small, unproblematic, but it is critical if either $T_{\text {ser }}$ or $n$ are bigger (Amdahl's Law).

Example 7.1.5. In the case of $T_{\text {ser }}=0.2$ and $n=8$ the total execution time reduces to $T(8)=0.2+\frac{0.8}{8}=0.3$ resulting in a speed-up of $S p(8)=3 . \overline{3}$ which is far from the expected ideal speed-up of $S p_{\text {opt }}(8)=8$.

Remark 7.1.6. In addition to the serial part $T_{\text {ser }}$ of the algorithm the parallel overhead, such as synchronization and data exchange, slows down the parallel execution of the program.

But a gleam of hope is spotted in the fact that the non-parallelizable part $T_{\text {ser }}$ generally decreases with increasing size of the problem (Gustafson's Law). Thus, even with bad performance with respect to speed-up $S p(n)$ the algorithm can still possess a good scalability $S c(n)$.

For further details cf. [SM95, Sch99, TG99, vdV94].

\subsection{Parallelization Concepts}

In recent years two different types of supercomputers have become widely available, namely the shared memory architecture and the distributed memory architecture. As these two types of architecture also influence the efficiency of the parallelization strategy, they are now described in further detail.

\subsubsection{Distributed Memory Architectures}

In this approach the parallel computer consists of many individual processors, each possessing its own memory. One processor cannot access the memory of another. An executed program and its processed data is split into $n$ parts and is distributed to the $n$ processors. An exchange of information is possible via a communication network connecting the processors with each other (see e.g. [DRSW04]). A fast network, with short length of path from one processor to any other one, is essential for the performance of such a machine. This is also the limiting factor of the size of such supercomputers which can, nevertheless, consist of up to thousands of processors.

For the information transfer on such architectures there exists a widespread standard, the message passing interface MPI (see [Mpi97]).

\footnotetext{
${ }^{1}$ A possible exception to this rule is the improved cache performance for smaller problems.
} 


\subsubsection{Shared Memory Architectures}

This type of parallel computer also consists of individual processors but each of them has access to the same memory. An exchange of information is therefore easily realized by the common memory, an explicit, time consuming, message passing is not necessary. This advantage is diminished by the risk of the, so-called, race condition, i.e. two processors modifying the same storage position. The outcome of such a situation is non-deterministic and has to be avoided by the programmer. Also, after changing a common variable it is necessary to update the cache of every processor keeping this variable. For further details confer Section 7.3.

For hardware considerations this architecture is more difficult to realize than the distributed memory approach. The requirements for the fast memory access via the memory switch limit the size of such an architecture (nowadays) to some hundred processors.

The accepted programming standard for the usage of shared memory computers is the OpenMP standard, as described in [Ope02] and briefly in Section 7.3.

\subsubsection{Hybrid Architectures}

These are parallel computers sharing the characteristics of both of the above described architectures. The largest supercomputers these days are of this type. They consist of many nodes connected to each other by a communication network, whereas each node is composed of processors accessing one memory block within their node.

In the programming model both types of directives, MPI as well as OpenMP, are used.

\subsection{The OpenMP Parallelization}

\subsubsection{The Finite Volume Scheme}

The parallelization of the main numerical finite volume scheme is quite straightforward.

First, the mesh is divided into $n_{\text {part }}$ disjoint domains $\mathcal{D}_{i}\left(1 \leq i \leq n_{\text {part }}\right)$, called partitioning. The objective for the creation of these domains is an equal distribution of elements. This is necessary for the even distribution of the load between the processors (further clarified in Section 7.5 on page 105). Furthermore, small boundaries to other domains are desirable, due to the fact that the data $\mathbf{U}_{j}$ on each element situated on a domain-boundary can be modified by two processors ${ }^{2}$. Thus, in order to avoid the above-mentioned race condition, this element has to be locked before changing the content of $\mathbf{U}_{j}$ and unlocked afterwards. As this locking/unlocking needs time for itself, and might block the other processor, this should be avoided as much as possible. The partitioning is performed by the recursive coordinate bisection algorithm. It is fast, easy to implement, and efficient regarding the above required attributes (cf. [Kal96a, Sch99]). In order to handle the diverse add-ons to the main

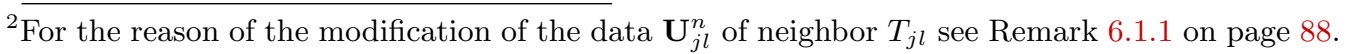


numerical scheme, this recursive coordinate bisection had to be extended, which is described in Section 7.4.

Then the OpenMP \#pragma omp parallel-directive is applied to create $n$ different threads (each being run on one processor) which execute the same code from this point onwards. Thus, each thread $i$ calculates the same scheme on all elements $T_{j} \in \mathcal{D}_{i}$ of the domain $\mathcal{D}_{i}$ that has been appointed to it.

For synchronization of the threads the directive \#pragma omp barrier is used. The threads wait at this point for each other. This is, e.g., necessary at the end of one time-step, before the threads use the data $\mathbf{U}_{j l}^{n-1}$ of the neighboring elements from the time-step before. It has to be assured that this data is completely calculated.

For the update of global variables it is crucial that only one thread at a time accesses this variable to avoid the race condition. This is done by the \#pragma omp critical-directive. E.g. for the new minimum time-step $\Delta t_{\text {min }}$ each thread $i$ calculates its own local minimum time-step $\Delta t_{i \text {,min }}$ on domain $\mathcal{D}_{i}$ and compares it with the global $\Delta t_{\min }$. If $\Delta t_{i, \min }<\Delta t_{\min }$ then $\Delta t_{\min }:=\Delta t_{i, \min }$, using this directive.

A similar case is given for instructions that only one thread needs to execute. They are indicated by the \#pragma omp single.

And finally, the locking of the data $\mathbf{U}_{j}^{n}$ that can be modified by more than one thread (if the element $T_{j}$ is situated at a domain boundary) is done by the OpenMP library functions omp_set_lock() and omp_unset_lock().

The changes that need to be applied to the serial program are minor, such that the same code can be used for serial and parallel execution. The dedication is set at compilation time by the compiler flag -OPENMP.

\subsubsection{The Mesh Routines}

The parallelization of the main scheme resulted in a code which had a rather big serial part $T_{\text {ser }}$, most importantly due to the routines for the mesh movement and the dynamic local mesh refinement. Therefore, the speed-up and efficiency of this parallel code was not very impressive. To enhance the performance two strategies were applied.

On the one hand, the absolute execution time of the mesh related routines was optimized. This was achieved by profiling and tuning the concerned program parts (cf. Section 10.8 on page 195), and by changing the mesh not after each step but only after $2^{l_{\text {geom }}}$ steps, with $l_{\text {geom }}$ as a given parameter (cf. Section 6.1 on page 87 ).

On the other hand, these routines used for mesh treatment were parallelized to the greatest possible extent. For this, we used not just one partitioning of the mesh for the finite volume scheme, as described in Paragraph 7.3.1 on the page before, but in parallel also several others for the mesh related routines (see Section 7.4).

\subsection{The Extended Partitioning Algorithm}

The load of a single processor during the execution of the finite volume scheme is determined by the number of elements being updated at the current time-step $t^{n}$. Because of the adaptive local time-stepping (cf. Section 6.1 on page 87 ) this number is different for each time-step level $l_{\text {timestep }}$. An element $T_{j_{1}}$ belonging to time-step level $l_{j_{1}}=l_{\min }$ is calculated every time-step, one element $T_{j_{2}}$ belonging to the next 
higher level $l_{j_{2}}=l_{\min }+1$ every second step, and so on. Thus, for a time-step in which only elements of level $l_{\text {timestep }}=l_{\text {min }}$ are updated, only these elements contribute to the load in this step. In a time-step for level $l_{\text {timestep }}=l_{\min }+1$ all elements of level $l_{j} \leq l_{\min }+1$ are to be considered, and so forth. Therefore, we need for each level $l_{\text {min }} \leq l_{\text {timestep }} \leq l_{\max }$ a separate partitioning in order to obtain a correct load balancing for every single time-step. That means that the domains $\mathcal{D}_{i}^{l_{k}}(k=1,2)$ can differ for $l_{1} \neq l_{2}$.

Additionally, the mesh routines need to work in parallel, too. For the snapper algorithm, e.g., most of the time only one layer of elements in the cylinder and the crankcase are changed (see Section 5.2 on page 72). Thus, the elements in this layer have to be partitioned for the $n_{\text {part }}$ processors on the fly. Similarly, for the treatment of the rotating crankshaft mesh we need a balanced partitioning of this part of the mesh. The dynamic local mesh adaption algorithm (described in Section 6.2 on page 91) is operating on every cell, thus a further partitioning of the whole mesh is necessary. As the number of elements on domain boundaries is not important in the case of the mesh routines (no data on neighboring elements needs to be changed, thus no locking is necessary) a simple partitioning by element number is carried out. Summarizing this, we obtain a whole family of partitions $\mathcal{P}^{r}:=\left\{\mathcal{D}_{i}^{r} \mid 1 \leq i \leq n_{\text {part }}\right\}$ with $1 \leq r \leq l_{\max }-l_{\min }+4$ with each partitioning having a particular role within the load balancing procedure.

This approach therefore uses the capabilities of the shared memory architecture to its full extent, since a new partitioning does not need any communication or data exchange as it would be the case in distributed memory machines. On this distributed memory architecture the above described approach of using more than one partitioning would be impossible.

\subsection{Dynamic Load Balancing}

The load balancing in our case is a very important issue. As the mesh is changed by several different mechanisms, the size of the partitioning domains $\mathcal{D}_{i}^{r}$ is also affected. However, if these domains are not evenly balanced the performance of the parallelization suffers, since some processors have a lower work load, waiting for the others to finish (cf. [DRSW04]). Therefore, a dynamic load balancing is essential.

For this, a concept of work load has to be derived (see also [Sch99]). It is assumed that the work load is proportional to the number of elements that a processor has to compute in a certain time-step level. It follows for the cost per level for each domain $\mathcal{D}_{i}$ :

$$
W(l, i):=m_{l i} 2^{-l}
$$

with $m_{l i}$ being the number of elements $T_{j}$ in domain $\mathcal{D}_{i}$ with $l_{j} \leq l$. The total cost for a complete level cycle is thus:

$$
W:=\sum_{l_{\min } \leq l \leq l_{\max }} \max _{i}(W(l, i))
$$

Every $2^{l_{\text {part }}}$ steps, with $l_{\text {part }} \in \mathbb{N}$ a given constant, the even distribution of the domains is verified by calculating the ratio of the number of elements of the biggest 
to the smallest domain:

$$
\zeta_{\text {loadbal }}^{n}:=\frac{\sum_{l_{\min } \leq l \leq l_{\max }} \max _{i} W(l, i) \frac{\max _{i}\left(m_{l i}\right)}{\min _{i}\left(m_{l i}\right)}}{W}
$$

In the case of exceeding a given threshold $C_{\text {loadbal }}>1$ a new partitioning, i.e. a re-distribution of the mesh to the processors, is created by the extended recursive coordinate bisection, as described in Paragraph 7.4 on page 104 . 


\section{Chapter 8}

\section{The Connecting Rod - 2D Estimations}

\subsection{Description of the Problem}

As stated in Section 5.4 on page 85, the movement of the connecting rod is a complicated three-dimensional tilting/shifting mechanism. Its position interferes with the piston movement and the crankshaft rotation. Thus, for a direct simulation within our existing software a complete new technique would be needed. Hence, the question, to what extent the movement of the connecting rod influences the flow pattern in the cylinder at the scavenging process, has been analyzed using a quasi two-dimensional model of the crankcase. If the influence can be neglected in two dimensions then this small but complex structure can probably be omitted in the three-dimensional case, where additionally the rotation of the much bigger crankshaft overlays the influence of the connecting rod at least partially, thus decreasing this influence even more.

A detailed description of the adaption of the algorithm in order to calculate this problem is given in the following.

\subsection{The Model}

For the quasi two-dimensional simulation, a cross-section of the crankcase has been used, such that the inlet duct, the connecting rod and the transfer ports could be taken into consideration (see Figure 8.3.1 on page 111). All geometrical sizes have been extracted from the CAD data of the real-world engine and all physical dimensions have been respected. The meshes had a 3D structure but were only one layer of elements thick, thus the structure of the numerical solution was essentially two-dimensional.

As the temperature differences in the crankcase are by far not as dramatic as in the cylinder no temperature dependencies of the gas properties (described in Section 3.8 on page 40) were included in this simulation. 


\subsubsection{The Compression of the Crankcase}

The compression and expansion of the crankcase by the motion of the piston, which was not directly simulated due to the quasi two-dimensional setting, was modeled. This was done by modifying the pressure $p_{j}^{n}$ of each element $T_{j}$ within the crankcase mesh during the time-stepping iteration according to the change of volume (as depicted on the left-hand side of Figure 8.2.1 on the facing page)

$$
V^{n}:=V\left(t^{n}\right):=110.5+59.0 \frac{1+\cos \left(\theta\left(t^{n}\right)\right)}{2},
$$

$\theta\left(t^{n}\right)$ being the crankshaft angle at time $t^{n}$, in an adiabatic manner:

$$
\tilde{p}_{j}^{n}:=p_{j}^{n}\left(\frac{V^{n}}{V^{n+1}}\right)^{\gamma}
$$

Thus, the finite volume scheme has been split, as in Paragraph 5.3.3 on page 83,

$$
\begin{aligned}
\tilde{\mathbf{U}}_{j}^{n}= & \left(\begin{array}{c}
\rho_{j}^{n} \\
\left(\rho v_{1}\right)_{j}^{n} \\
\left(\rho v_{2}\right)_{j}^{n} \\
\left(\rho v_{3}\right)_{j}^{n} \\
e\left(\tilde{p}_{j}^{n}\right)
\end{array}\right) \\
\mathbf{U}_{j}^{n+1}= & \tilde{\mathbf{U}}_{j}^{n}-\frac{\Delta t^{n}}{\left|T_{j}\right|}\left(\sum_{l=1}^{k_{j}} \mathbf{g}_{j l}\left(\tilde{\mathbf{U}}_{j}^{n}, \tilde{\mathbf{U}}_{j l}^{n}\right)-\sum_{l=1}^{k_{j}} \mathbf{G}_{j l}\left(\tilde{\mathbf{U}}_{j}^{n}, \tilde{\mathbf{U}}_{j l}^{n}\right)\right)
\end{aligned}
$$

where $e\left(\tilde{p}_{j}^{n}\right)$ is calculated via the thermal and caloric equation of state (cf. Section 3.5 on page 36$)$ to represent the new pressure $\tilde{p}_{j}^{n}$.

\subsubsection{The Inlet and Outlet Boundary Conditions}

Also, the opening and closing of the inlet duct and the transfer ports by the piston had to be modeled, since the motion of the piston was not directly simulated, as stated above. This has been accomplished by an approximation of the open window area at the end of the ports by a multiplication factor of

$$
\begin{aligned}
C_{\text {inlet }}\left(t^{n}\right) & :=\max \left\{0,1.4827295\left(\cos \left(\theta\left(t^{n}\right)\right)-0.32556815\right)\right\} \text { and } \\
C_{\text {transfer }}\left(t^{n}\right) & :=\frac{3.393}{7.540} \max \left\{0,-2.9074757\left(\cos \left(\theta\left(t^{n}\right)\right)+.65605903\right)\right\},
\end{aligned}
$$

for the inlet and transfer ports respectively (see the lefthand side of Figure 8.2.2 on the next page). The mesh extension in $z$-direction was chosen such that the maximum window area for the inlet port was identical to the three-dimensional CAD data. Thus, the timing of opening and closing of inlet and transfer ports corresponds exactly to the three-dimensional setting, as well as the maximum window area. Just the exact shape of the ports has been approximated by a rectangular cross-section of the ducts resulting in the above presented formulae. 
As artificial boundary condition we used, as in the 3D case, a mass-flow condition at the inflow boundary (see Figure 8.2.1 (right)). At the outflow boundary at the end of the transfer ports, the periodically changing pressure in the cylinder was prescribed (as shown in Figure 8.2.2 (right)). This pressure was obtained by a onedimensional gas-exchange simulation of the engine, which is reliable for this kind of information.

\subsubsection{The Motion of the Connecting Rod}

The shape of the cross-section of the connecting rod can be seen on Figure 8.3.1 on page 111. It has been taken directly from the CAD data, as well. Also, the maximum amplitude of the oscillation of the connecting rod corresponds to the real dimensions of the 3D case. The complex horizontal oscillation $\xi_{\text {rod }}(t)$ itself has been modeled by (see Figure 8.2.3 on the next page)

$$
\xi_{\text {rod }}\left(t^{n}\right):=\xi_{\text {max }} \sin \left(\theta\left(t^{n}\right)\right) .
$$
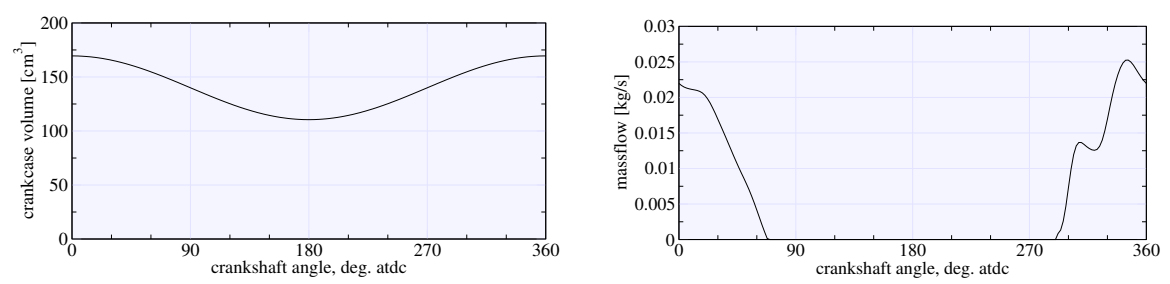

Figure 8.2.1: Modeled crankcase volume (left) and massflow boundary condition at the inlet (right).
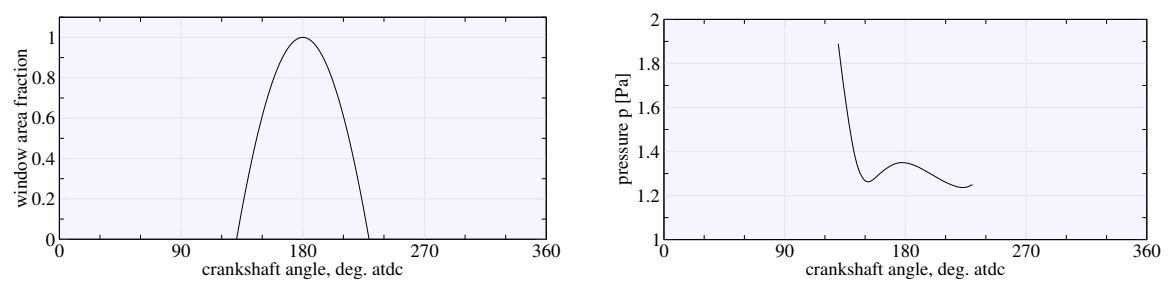

Figure 8.2.2: Window area fraction (left) and pressure boundary condition (right) at the end of the transfer ports.

\subsection{Meshes}

To be able to estimate the influence of the connecting rod, geometries with and without it were created. In order to judge the mesh dependency of the results, 

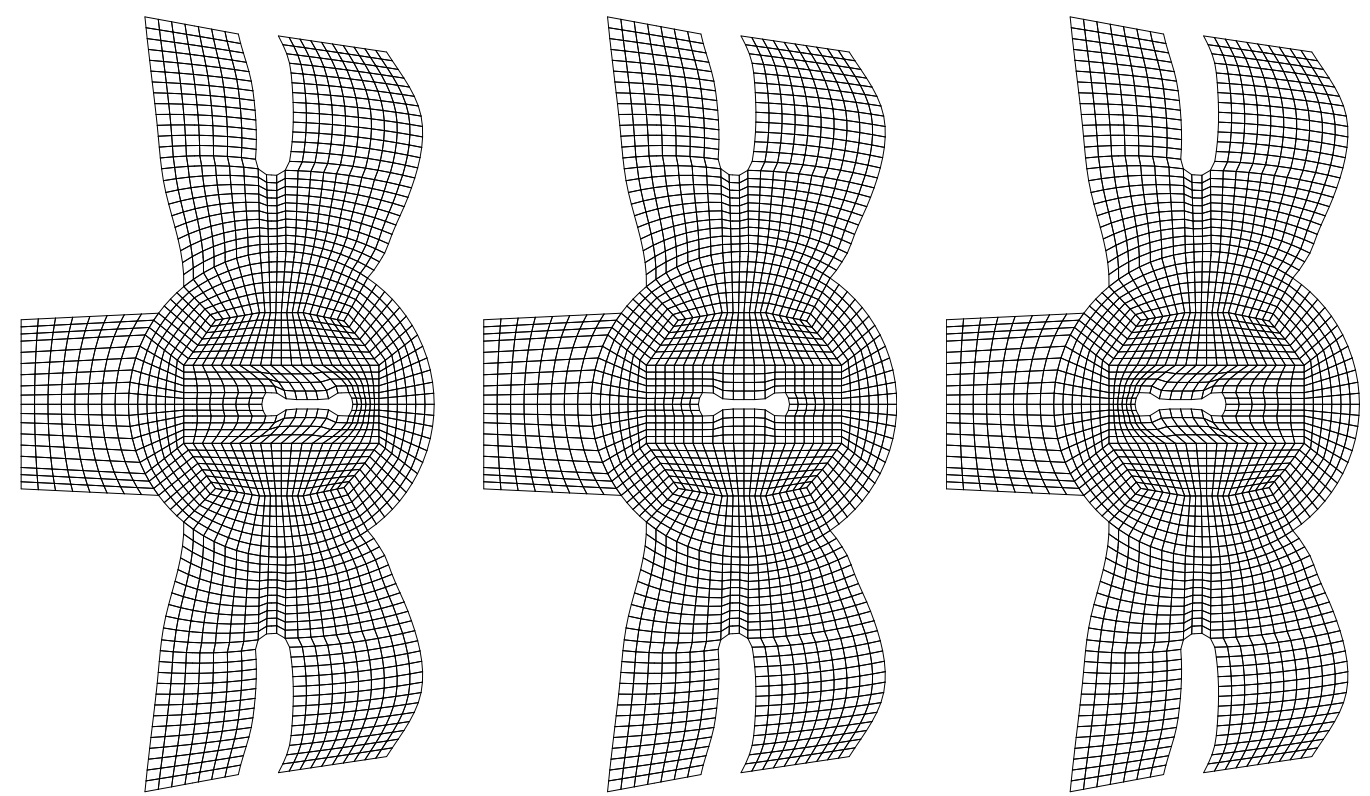

Figure 8.2.3: Movement of the connecting rod: at $90^{\circ}$ (left), at $180^{\circ}$ (middle), and at $270^{\circ}$ crankshaft angle (right).

meshes with 1000, 2000, 4000, $8000,16000,32000,64000$, and 128000 elements for both geometries were generated. Figure 8.3.1 on the facing page shows the meshes with 4000 elements for the two geometries. As stated above, the meshes are three-dimensional but only one layer of elements thick. With the boundary condition being a slip condition at the upper and lower face (cf. Sections 3.4 on page 32 and 4.7 on page 55), the numerical solution was not influenced by the third dimension and behaved just as in a two-dimensional simulation.

\subsection{Results}

A convergence in time in the sense of (6.3.1) was achieved on each mesh by the iterative process described in Paragraph 6.3.3 on page 99. For the coarsest meshes about ten periods were needed (see the left-hand side of Figure 8.4.1 on the facing page), for the finer ones only about three periods due to the iterative process of Paragraph 6.3 .3 on page 99 .

Now, the average velocity at the end of a transfer port was taken as an indicator of the influence of the connecting rod on the flow entering the cylinder. The average differences of this velocity in the calculations with and without the connecting rod were plotted (Figure 8.4.1 (right)) to analyze this influence. It can be seen that the difference between the two configurations is below $2 \%\left(\max .0 .38 \frac{\mathrm{m}}{\mathrm{s}}\right.$ at an average velocity of $25.4 \frac{\mathrm{m}}{\mathrm{s}}$ ) and this even decreases on the finer meshes.

In Figure 8.4.2 on page 112 the density and velocity profiles for the two configurations at two different times are shown. The influence of the connecting rod on this 2D flow pattern is apparent. However, at opening of the transfer ports (in 

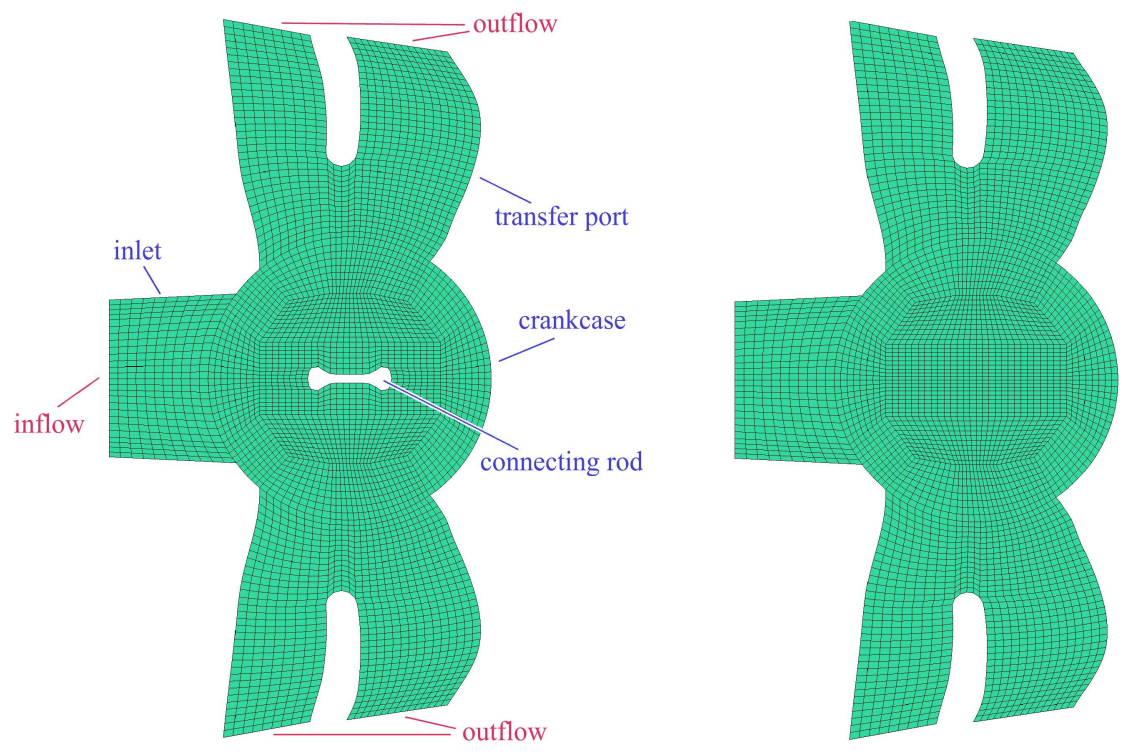

Figure 8.3.1: Quasi-2D mesh with (left) and without (right) connecting rod (4000 elements, each).

the bottom pictures) at the end of the transfer ports (their connecting point to the cylinder), the flow patterns of the two configurations coincide very well. This coincidence indicates the diminishing influence of the connecting rod for the flow reaching the cylinder.

Hence, according to this investigation, we can conclude that the connecting rod, which is in the $3 \mathrm{D}$ case an even less dominating structure than in the $2 \mathrm{D}$ one, is not important for the simulation of the scavenging process.

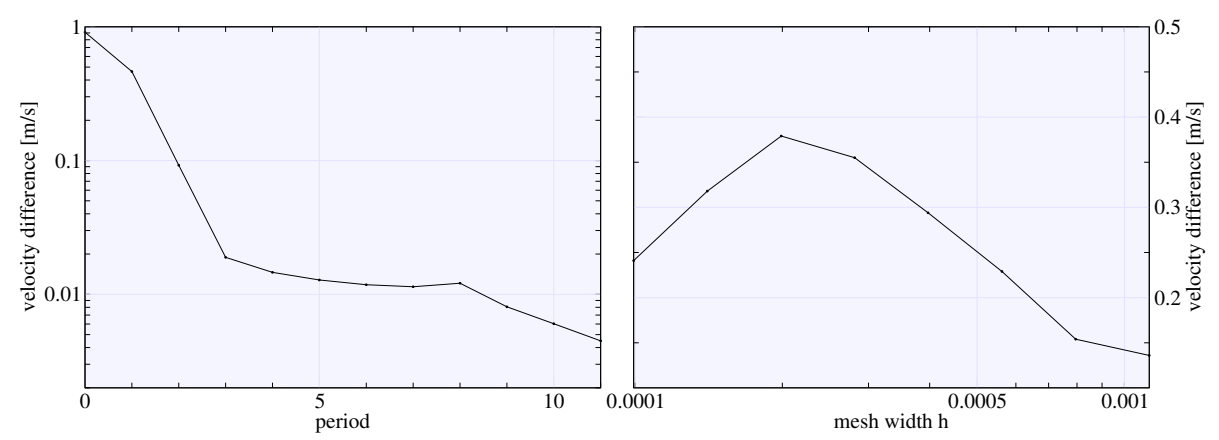

Figure 8.4.1: Temporal convergence history of the average velocity difference at the end of a transfer port between two periods (left), and mesh dependence history of the average velocity difference of velocity at the end of a transfer port on the different meshes (right). 

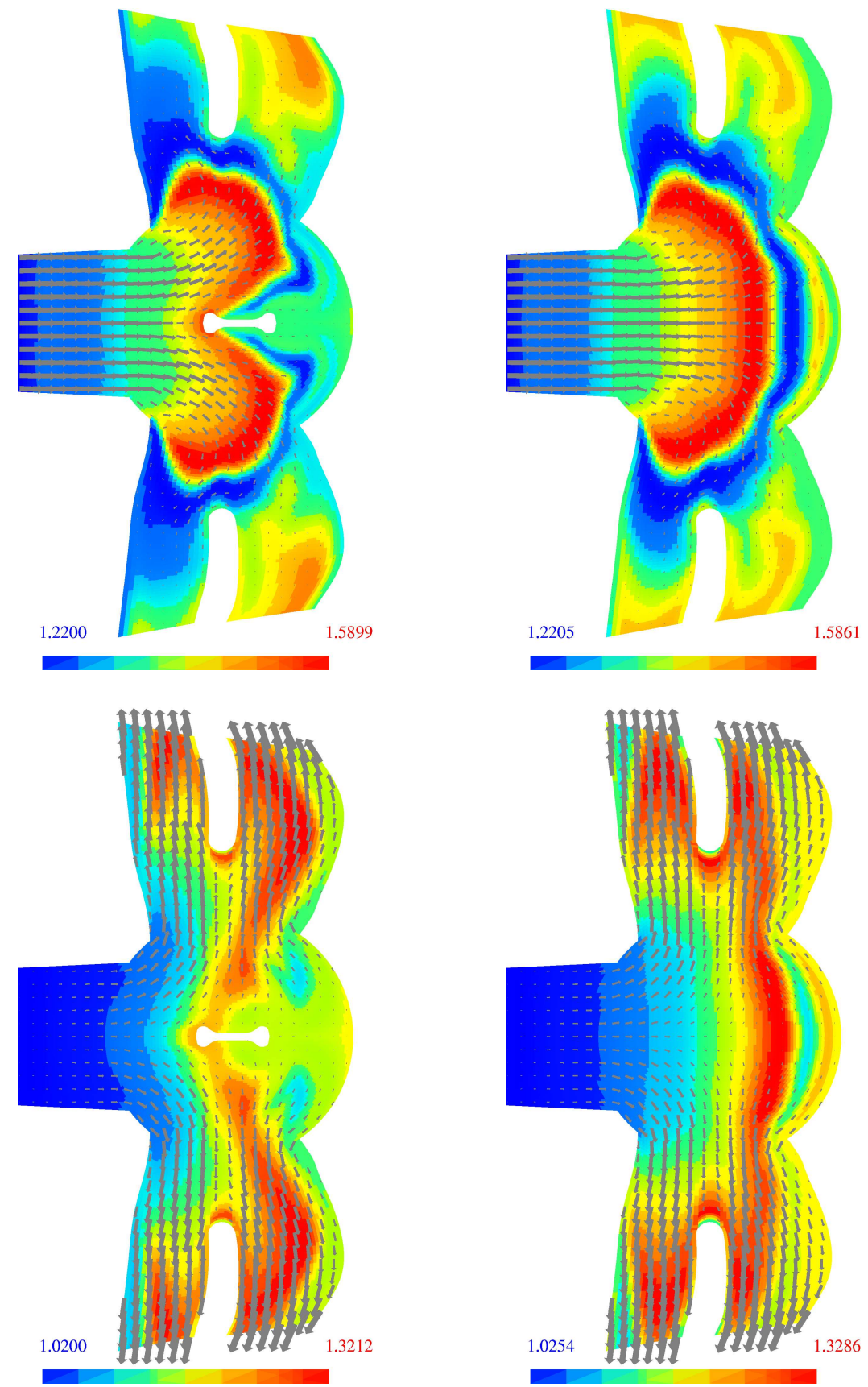

Figure 8.4.2: Structure of the flow at $30^{\circ}$ crankshaft angle (top) and $170^{\circ}$ crankshaft angle (bottom) with (left) and without (right) connecting rod (density and velocity are shown). 


\section{Chapter 9}

\section{Validation of the Software}

\subsection{Evaluation of a Numerical Scheme}

For simple model problems, the convergence properties of a finite volume scheme can be studied analytically (see e.g. [Krö97]). However, in our case of the threedimensional time-dependent Navier-Stokes equations, the numerical scheme has to be evaluated by numerical experiments. The results of these calculations can then be compared to known exact solutions, calculations with other schemes found in literature, or physical experiments. All of these methods were conducted and the results are described in the following.

\subsubsection{The Experimental Order of Convergence}

For the study of the convergence rate of a numerical scheme, and as a good indicator for convergence, the experimental order of convergence can be used. This concept is based on the assumption that the error of a numerical scheme can be expressed in powers of the mesh width $h$ :

$$
\left\|u-u_{h}\right\|_{\Omega}=C h^{\alpha}+O\left(h^{\alpha+1}\right)
$$

with the constant $C$ independent of $h$.

Definition 9.1.1. (EOC) Let $\mathbf{U}: \Omega \times \mathbb{R}^{+} \rightarrow \mathbb{R}^{K}$ be the exact solution of a system of conservation laws, and $\mathbf{U}_{h_{1}}, \mathbf{U}_{h_{2}}: \Omega \times \mathbb{R}^{+} \rightarrow \mathbb{R}^{K}$ two numerical solutions calculated with the numerical scheme in question on two different grids with minimal mesh width $h_{1}$ and $h_{2}$ respectively. Then the experimental order of convergence (EOC) of the numerical scheme is defined by

$$
\text { EOC }:=\frac{\ln \left(\left\|(\mathbf{U})_{i}-\left(\mathbf{U}_{h_{1}}\right)_{i}\right\|_{\Omega}\right)-\ln \left(\left\|(\mathbf{U})_{i}-\left(\mathbf{U}_{h_{2}}\right)_{i}\right\|_{\Omega}\right)}{\ln \left(h_{1}\right)-\ln \left(h_{2}\right)}
$$

with $(\cdot)_{i}(i \in\{1, \ldots, K\})$ indicating the $i^{\text {th }}$ component of the solution, and a suitable norm $\|\cdot\|_{\Omega}$.

Remark 9.1.2. The $L_{1}$-norm has been used to evaluate the numerical errors in the definition above. As we employed a numerical scheme of first order, this $L_{1}$-norm 
can be approximated by

$$
\left\|(\mathbf{U})_{i}-\left(\mathbf{U}_{h}\right)_{i}\right\|_{L^{1}} \approx \sum_{T_{j} \in \mathcal{T}}\left|T_{j}\right|\left|\left(\mathbf{U}\left(\mathbf{w}_{j}\right)\right)_{i}-\left(\mathbf{U}_{h}\left(\mathbf{w}_{j}\right)\right)_{i}\right|
$$

where $\mathbf{w}_{j}$ is the center of gravity of element $T_{j}$ (see Section 4.2 on page 46 ).

Remark 9.1.3. It could not be observed that the EOC was influenced by the step size $\Delta t$, as long as the CFL-condition was satisfied. Therefore, the adaptive local time-stepping was used in all test problems.

Remark 9.1.4. For a theoretical discussion on the convergence rate of a numerical scheme for systems of hyperbolic conservation laws see e.g. [LeV90].

\subsubsection{The Efficiency of a Scheme}

The EOC of a numerical scheme gives no information about the computing time necessary on a given mesh nor the actual error on that mesh. Therefore, the efficiency of a scheme, measured by the runtime to reach a given error, is probably even more important than its EOC if we want to compare two different numerical approaches. Two schemes can have a very different efficiency even if they are of the same order. This is due to different constants $C$ in equation (9.1.1), i.e. a different absolute error on the same mesh, and a different computing time to obtain the solution.

Also, the superior convergence of higher order schemes might only be experienced on grids with small mesh sizes.

\subsection{Inviscid Flow}

As we want to study the performance of the different modules of our software we need test cases for these specific parts. In this section the convective part of our numerical scheme is tested with inviscid flow problems. The mathematical model of this flow is given by the compressible Euler equations of gas dynamics, as described in Section 3.3.1 on page 31. The numerical scheme is described in detail in Sections 4.3 on page 47, 4.7, and 4.8, with the convective flux from Section 4.4 on page 50. All the values from the diagrams are stated in the tables in Appendix A on page 205.

\subsubsection{Compression Profile}

In the first test problem a one-dimensional compression wave through a duct is considered (see Figure 9.2.1 on the facing page). This wave travels with a prescribed velocity and on its way the wave profile is distorted. On the wave front a so-called "steep-fronting" occurs (see Figure 9.2.2 on the next page). The crest of the wave travels faster than its front and tail. 


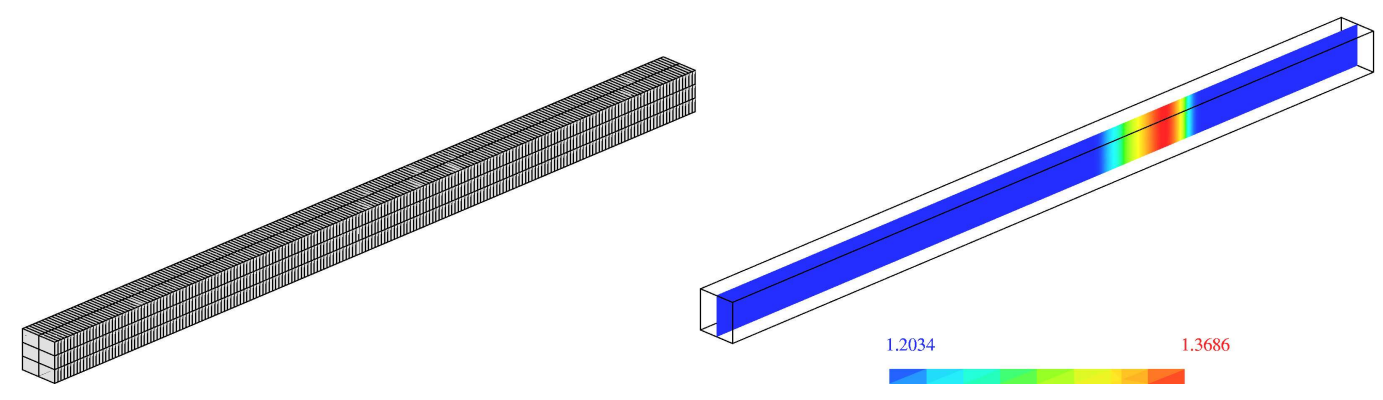

Figure 9.2.1: Compression profile: the $200 \times 3 \times 2$ element mesh (left) and a clipping plane of the calculated density at $t_{\text {end }}=0.01 \mathrm{~s}$ on the $3200 \times 3 \times 2$ element mesh (right) (the images have been scaled in $x$-direction by the factor 100).

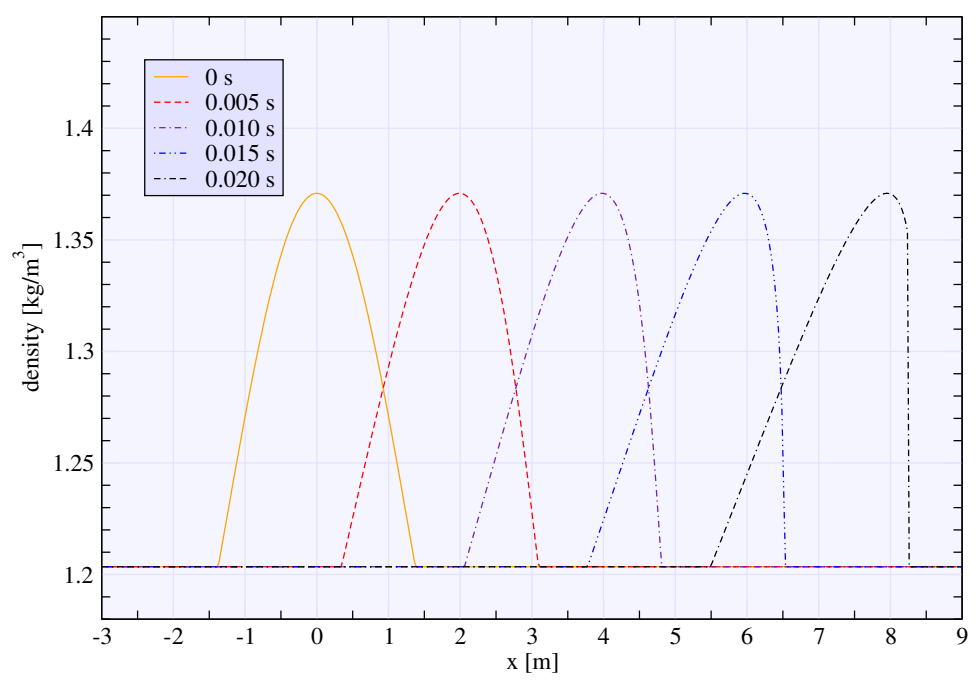

Figure 9.2.2: Compression profile: steep-fronting. 


\section{Exact Solution}

For a polytropic, i.e. calorically and thermally perfect, gas an exact solution of the compression profile can be calculated (as described in [LL95, Paragraph 101]) until the time $t=t_{\max }$ when the steep-fronting results in the formation of a shock wave. In order to obtain this exact solution, let the initial velocity profile be given by a function $F(x)$. Then the velocity $v$ can be written as

$$
v(x, t)=F\left(x-\left(c_{0}+\frac{\gamma+1}{2} v(x, t)\right) t\right)
$$

with $c_{0}=\sqrt{\frac{\gamma p_{0}}{\rho_{0}}}$ the speed of sound of the surrounding medium. ${ }^{1}$ With the help of this velocity $v$ the density $\rho$ and pressure $p$ are calculated as

$$
\rho(x, t)=\rho_{0}\left(1+\frac{\gamma-1}{2} \frac{v(x, t)}{c_{0}}\right)^{\frac{2}{\gamma-1}}
$$

and

$$
p(x, t)=p_{0}\left(1+\frac{\gamma-1}{2} \frac{v(x, t)}{c_{0}}\right)^{\frac{2 \gamma}{\gamma-1}} .
$$

\section{Setup of the Numerical Experiment}

In our test case the following values have been fixed (following the European standard temperature and pressure (STP) for the density $\rho_{0}$ and the pressure $\left.p_{0}\right)$ :

$$
\begin{aligned}
& \rho_{0}:=1.2039 \frac{\mathrm{kg}}{\mathrm{m}^{3}} \\
& p_{0}:=101325 \mathrm{~Pa} \\
& \gamma:=1.4 \\
& F(x):= \begin{cases}v_{\max } \cos \left(\pi \frac{x}{2.75}\right) & \text { for }-1.375 \leq x \leq 1.375 \\
0 & \text { otherwise }\end{cases} \\
& v_{\max }=\frac{2}{\gamma-1} c_{0}\left(\left(\frac{p_{\max }}{p_{0}}\right)^{\frac{\gamma-1}{2 \gamma}}-1\right) \\
& p_{\max }:=1.2 p_{0}
\end{aligned}
$$

$F(x)$ is chosen such that the compression pulse has a duration of about $8 \mathrm{~ms}$, which is an average port opening of a two-stroke engine running on 3000 RPM (cf. also test case 9.5.1 on page 158 and [B196]).

The computational domain is

$$
\Omega:=[-10 ; 10] \times[0 ; 0.01] \times[0 ; 0.01]
$$

with inflow and outflow boundary conditions at the two planes $P_{\text {in }}:=\{x=-10\}$ and $P_{\text {out }}:=\{x=10\}$ respectively, and slip wall boundary conditions on all other boundaries (cf. Figure 9.2.3 on the next page). The initial values in primitive

\footnotetext{
${ }^{1}$ This implicit equation (9.2.1) was solved with a damped fixpoint iteration. This method is feasible for $t$ not too big and appropriate functions $F(x)$ as given below.
} 
variables are given by $v(x, 0)=F(x), \rho(x, 0)$, and $p(x, 0)$ with $\rho(x, 0)$ and $p(x, 0)$ obtained by equations (9.2.2) and (9.2.3). ${ }^{2}$ The end time $t_{\text {end }}=0.01 \mathrm{~s}$ is chosen such that a shock has not been formed and no disturbing influence from the inflow and outflow boundary can occur.

To be able to calculate the convergence properties, different meshes have been generated, ranging from $100 \times 3 \times 2$ elements up to $12800 \times 3 \times 2$ elements (see Figure 9.2.1 on page 115 for a section of the $400 \times 3 \times 2$ element mesh $)^{3}$.

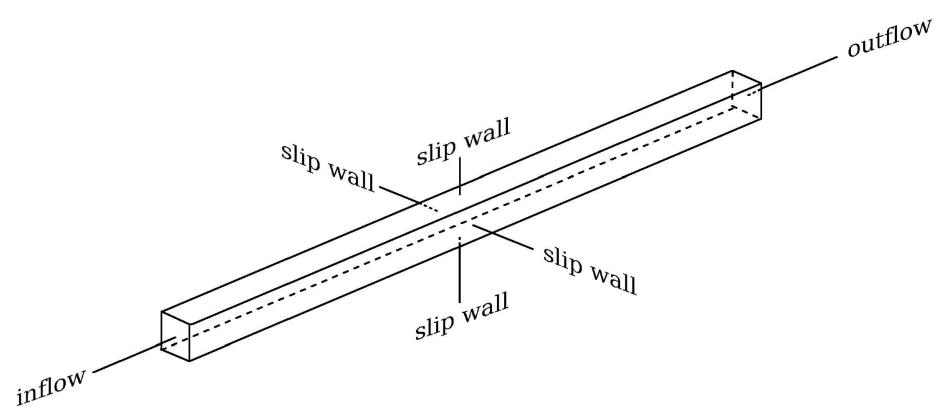

Figure 9.2.3: Compression profile: boundary conditions and domain $\Omega$ for the experimental setup.

\section{Results}

As can be seen in Figure 9.2.4 on the following page, on the finer meshes a very good approximation of the exact solution is attained. Especially the crest of the profile is rendered almost exactly on the finest mesh, and even the sharp changes at the front and tail are resolved with very good accuracy. The convergence history is shown in Figure 9.2.5 on the next page and in Table A.1 on page 205 in Appendix A. On each consecutive mesh the $L^{1}$-error (see Remark 9.1.2 on page 113) of the density is about half as big as before. This corresponds to an EOC of approximately 1.

Summing up, it has been shown that a compression profile, i.e. a continuous wave, can be simulated with very good accuracy on meshes that are well within the scope of a two-stroke engine simulation. Also, the convergence towards the exact solution is very satisfying.

\subsubsection{Sod's Shock Tube}

As next test case we consider the classical one-dimensional shock tube problem (see Figure 9.2.6 on page 119). The physical setup consists of a tube that is separated in two sections by a membrane. Each of the two sections is filled with gas of a

\footnotetext{
${ }^{2}$ Because of the independence of the initial values of the $y$ and $z$ coordinates and because of the above described boundary treatment, a quasi one-dimensional simulation is performed.

${ }^{3}$ On the finest mesh the length of a cell is approximately $1.5 \mathrm{~mm}$ which can be found in a medium sized macro grid of the two-stroke engine (cf. Section 10.1 on page 167).
} 


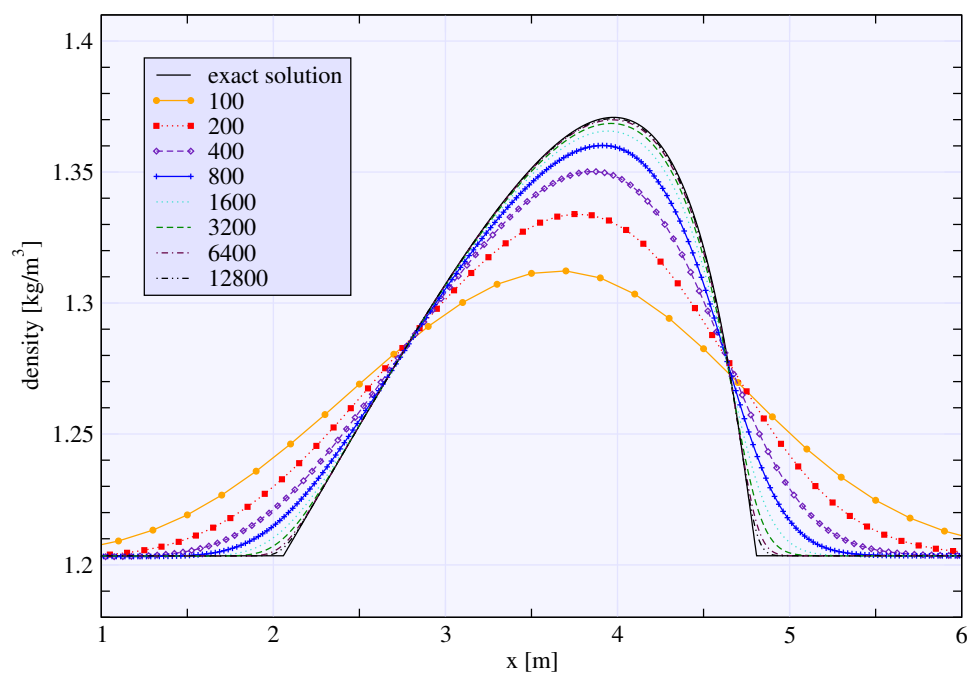

Figure 9.2.4: Compression profile: density on the different meshes at $t=0.01 \mathrm{~s}$.
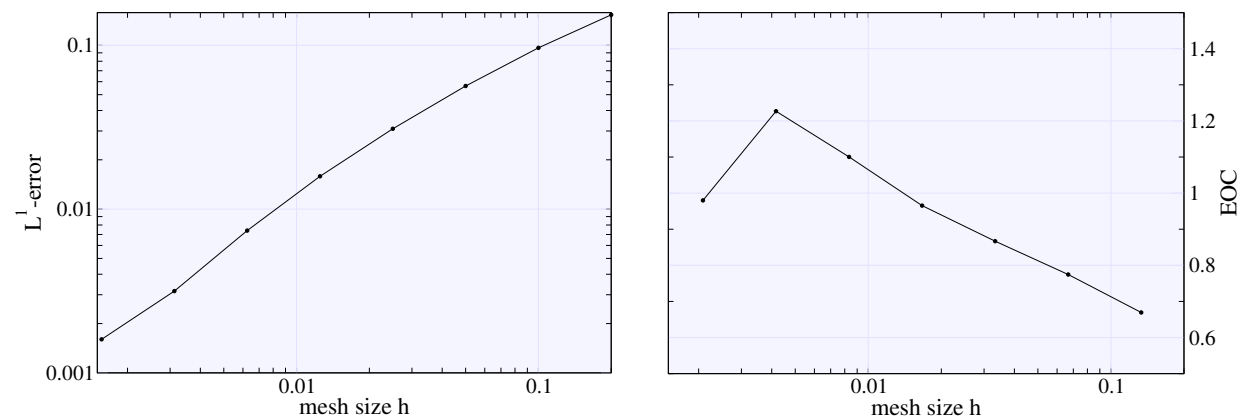

Figure 9.2.5: Compression profile: $L^{1}$-error (left) and EOC (right) evolution on the different meshes. 
different pressure and density. Initially the gas in both sections is at rest. At time $t=0$ the membrane is suddenly removed. Thus, this physical setup represents the one-dimensional Riemann problem defined by initial left and right-hand states $\mathbf{U}_{l}$ and $\mathbf{U}_{r}$ :

$$
\mathbf{U}_{0}(x):= \begin{cases}\mathbf{U}_{l} & \text { for } x<0 \\ \mathbf{U}_{r} & \text { for } x>0\end{cases}
$$

with $x \in \mathbb{R}$.

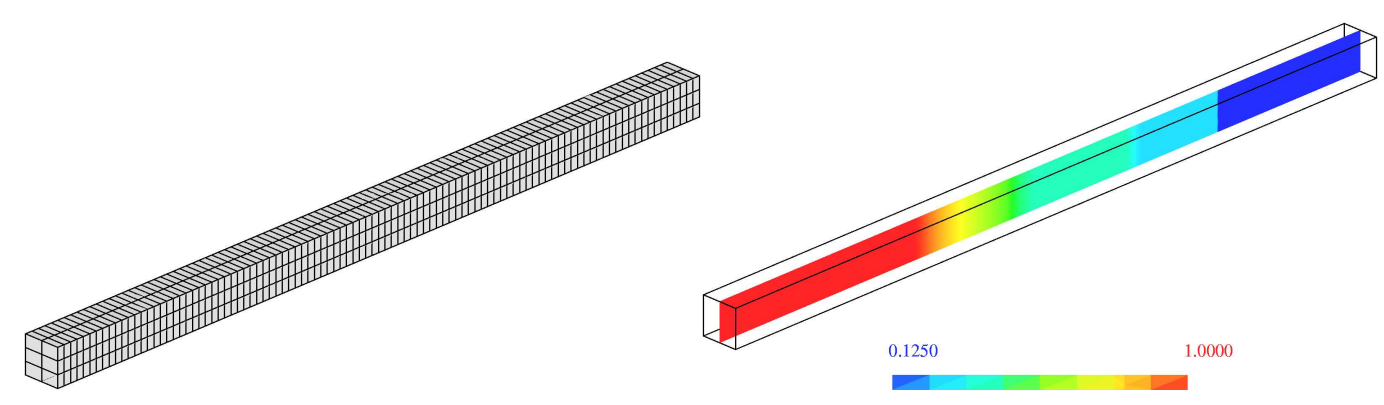

Figure 9.2.6: Shock tube: the $100 \times 3 \times 2$ element mesh (left) and a clipping plane of the calculated density at $t_{\mathrm{end}}=0.0001 \mathrm{~s}$ on the $3200 \times 3 \times 2$ element mesh (right).

\section{Exact Solution}

Like the compression wave in the test problem before, this is a time-dependent problem. Furthermore, an exact solution can be given in the case of a polytropic gas. It is composed of different constant states connected either by a rarefaction wave, a shock or a contact discontinuity (see e.g. [Krö97]). Chorin's method (cf. also [Krö97]) is used to reduce the one-dimensional Riemann problem to the solution of an ODE. For sufficiently small initial data (the jump from $\mathbf{U}_{l}$ to $\mathbf{U}_{r}$ must not be too big) the problem can be solved up to any given accuracy. Figure 9.2.8 on page 121 shows the exact solution for the time $t=0.0001 \mathrm{~s}$ of density, velocity and pressure for the below stated initial data.

\section{Setup of the Numerical Experiment}

The initial values in primitive variables $\left(\rho, v_{1}, p\right)^{T}$ (proposed by [Hir92b]) are given by:

$$
\begin{aligned}
\mathbf{U}_{l}:= & \left(\begin{array}{c}
1.0 \\
0.0 \\
100000
\end{array}\right) \\
\mathbf{U}_{r}:= & \left(\begin{array}{c}
0.125 \\
0.0 \\
10000
\end{array}\right)
\end{aligned}
$$

and $\gamma=1.4$ for the adiabatic exponent. 
The computational domain is

$$
\Omega:=[-0.1 ; 0.1] \times[0 ; 0.01] \times[0 ; 0.01]
$$

with inflow and outflow boundary conditions at the left and right hand side respectively, and slip wall boundary conditions on all other boundaries (cf. Figure 9.2.7). With this setup, as in the compression profile test case, a quasi one-dimensional simulation is performed, resulting in identical values of density, $\mathrm{x}$-velocity and pressure for arbitrary $y$ and $z$ coordinates.

The calculations are performed on meshes with $100 \times 3 \times 2$ elements up to $12800 \times$ $3 \times 2$ elements. ${ }^{4}$ (In Figure 9.2.6 on the page before the $400 \times 3 \times 2$ element mesh is shown.)

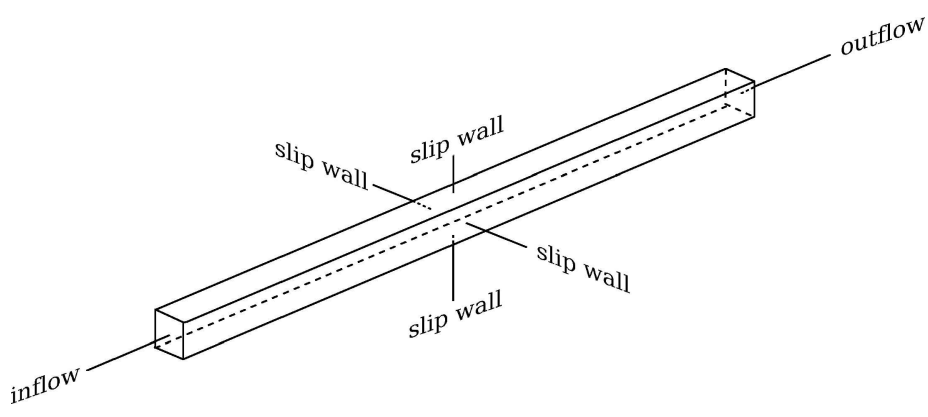

Figure 9.2.7: Shock tube: boundary conditions and domain $\Omega$ for the experimental setup.

\section{Results}

As the density profile represents the whole structure of the solution (expansion wave, contact discontinuity, and shock), in the following only the density is used to analyze the quality of the simulation. It can be seen in Figure 9.2.9 on the facing page that the approximation of the exact constant density states is very good and that even the connecting waves are captured well on the finer meshes, whereas the shock is better resolved than the contact discontinuity and the upper part of the expansion wave. The convergence to the exact solution is very smooth (see Figure 9.2.10 on page 122) which results in an almost straight line in the double logarithmic mesh size $-L^{1}$-error diagram. The EOC-values between 0.6 and 0.7 are lower than in the first test case, which can be expected from the presence of discontinuities (see also Remark 9.1.4 on page 114).

All together, it can be said that also in the case of even very strong expansion waves, contact discontinuities, and shocks a very satisfactory approximation of the exact solution on realistic meshes could be achieved.

\footnotetext{
${ }^{4}$ But because this time the computational domain is only $0.2 \mathrm{~m}$ long, the length of a single cell ranges from $2 \mathrm{~mm}$ down to $0.016 \mathrm{~mm}$. In the simulation of the two-stroke engine on the finest macro grid with maximum refinement a cell length of $0.2 \mathrm{~mm}$ can be achieved which is the equivalent of the $3200 \times 3 \times 2$ element mesh.
} 

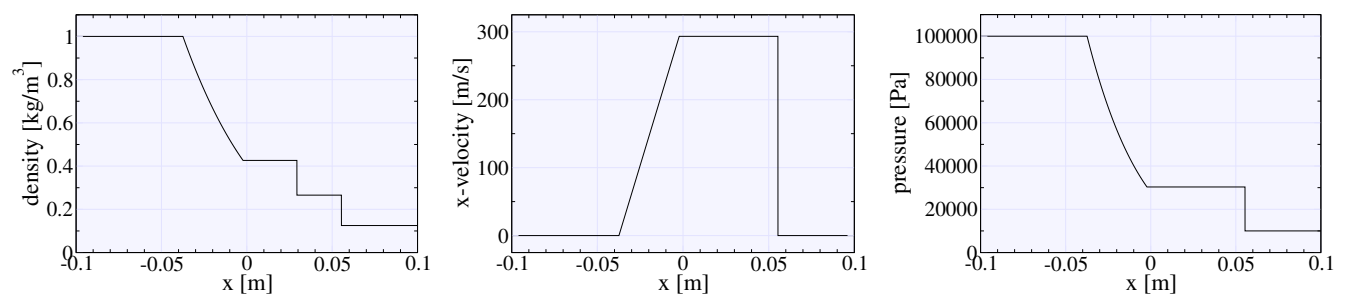

Figure 9.2.8: Shock tube: exact density (left), velocity in $\mathrm{x}$-direction (middle), and pressure (right) at $t_{\text {end }}=0.0001 \mathrm{~s}$.
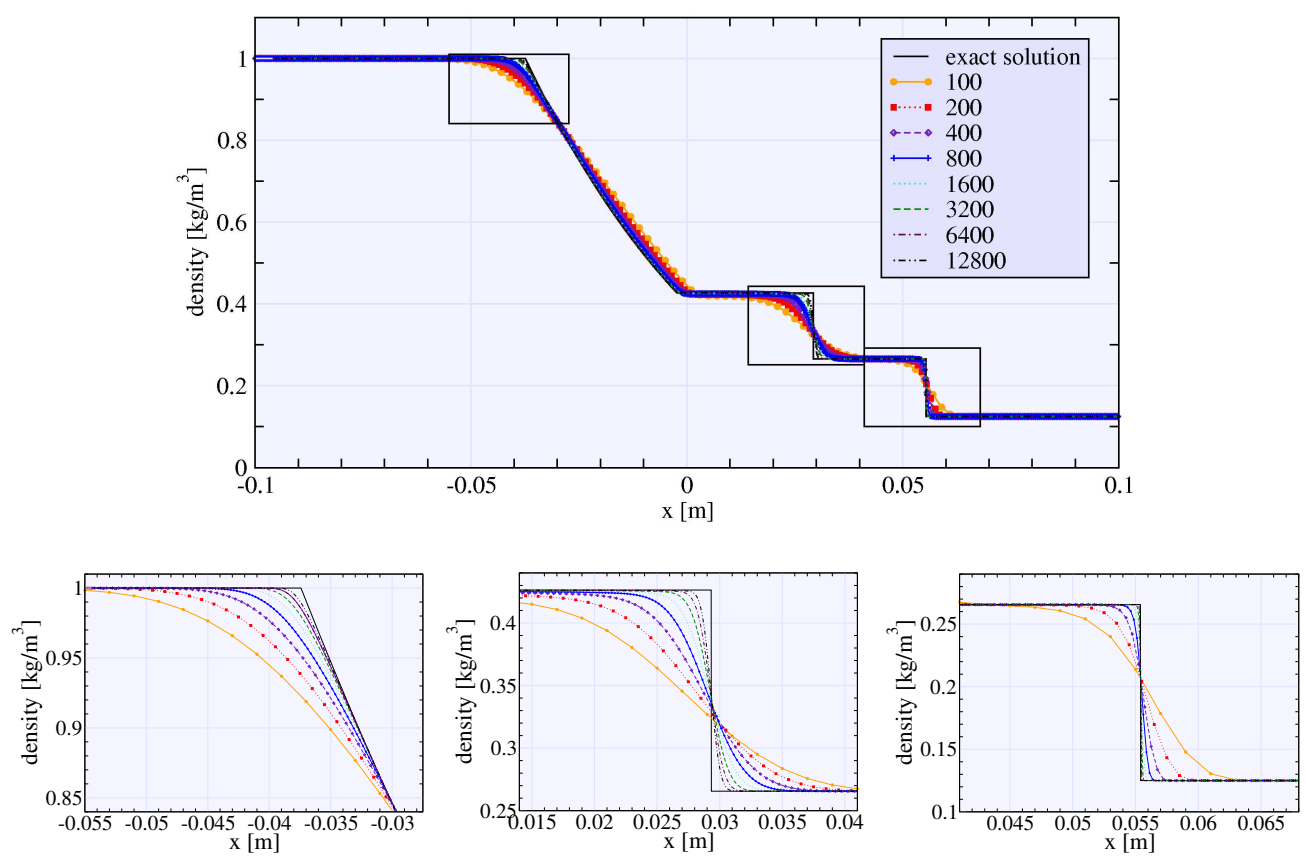

Figure 9.2.9: Shock tube: the whole solution (top) and detailed view (below) of the upper part of the expansion wave (left), the contact discontinuity (middle), and the shock (right) (displayed is the density profile along the tube on the different meshes and the exact density). 

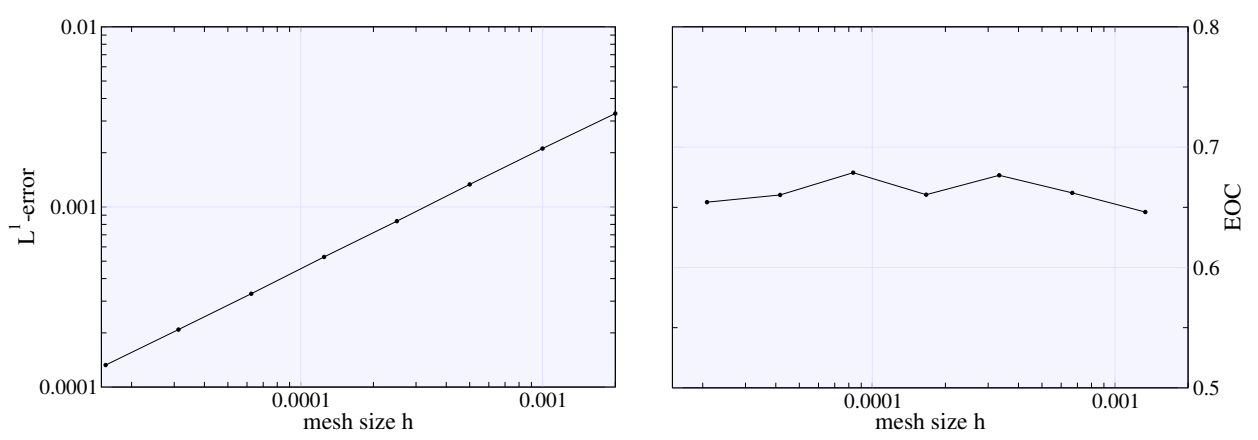

Figure 9.2.10: Shock tube: $L^{1}$-error of the density (left) and corresponding EOC (right) evolution on the different meshes.

\subsubsection{Nozzle Flow}

The third test problem with an inviscid regime is the two-dimensional nozzle flow (see Figure 9.2.11). A laminar flow passes a continuous expansion or compression of the cross-section of a duct. During this passage the values of pressure $p$, density $\rho$, and velocity $v$ of the flow undergo changes depending on the cross-section of the nozzle. Unlike the two test cases before, this is a stationary problem.

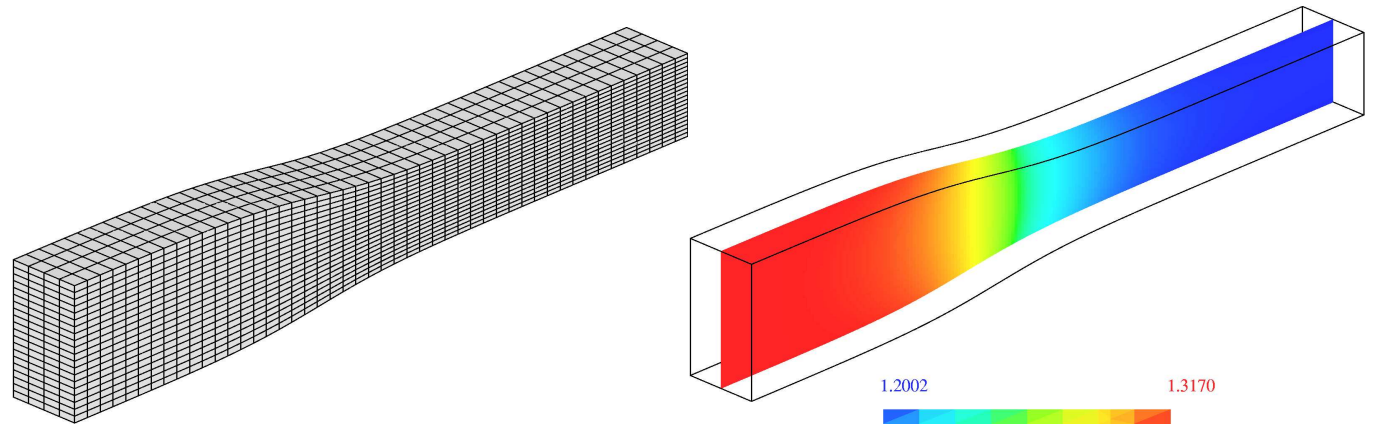

Figure 9.2.11: Nozzle flow: the $48 \times 20 \times 4$ element mesh (left) and a clipping plane of the calculated density on the $192 \times 80 \times 4$ element mesh (right).

\section{Exact Solution}

An exact solution for the nozzle flow for a polytropic gas can be given under the additional assumption that the velocity in orthogonal direction (here the velocity in $y$-direction) can be neglected. For a detailed derivation of the following equations see [LL95, Hir92b]. Let the cross-section $S(x)$, the critical cross-section $S^{*}$ (where the Mach number $M=1$ is reached), and the reference density and pressure $\rho_{0}$ and $p_{0}$ be given ${ }^{5}$. Also, it has to be fixed if a subsonic or supersonic regime is being

\footnotetext{
${ }^{5}$ The values $\rho=\rho_{0}$ and $p=p_{0}$ are theoretically reached in points with $M=0$ (see equation $(9.2 .5))$.
} 
considered. Here we restrict ourselves to the subsonic case. Then the Mach number $M(x)$ of the flow at position $x$ can be calculated by solving

$$
\frac{S(x)}{S^{*}}=\frac{1}{M(x)}\left(\frac{2}{\gamma+1}\left(1+\frac{\gamma-1}{2}(M(x))^{2}\right)\right)^{\frac{\gamma+1}{2 \gamma-2}} .
$$

The correct solution according to the subsonic or supersonic regime has to be chosen. ${ }^{6}$ Now $\rho(x)$ and $p(x)$ can be computed by

$$
\left(\frac{\rho_{0}}{\rho(x)}\right)^{\gamma-1}=\left(\frac{p_{0}}{p(x)}\right)^{\frac{\gamma-1}{\gamma}}=1+\frac{\gamma-1}{2}(M(x))^{2} .
$$

Therefore, we can obtain the local speed of sound

$$
c(x)=\sqrt{\frac{\gamma p(x)}{\rho(x)}},
$$

and finally the velocity

$$
v(x)=M(x) c(x)
$$

\section{Setup of the Numerical Experiment}

In our setup the parameters for a subsonic condenser flow are given $b y^{7}$ :

$$
\begin{aligned}
S(x) & :=0.01398+0.00347 \tanh (80(0.04-x)) \quad 0 \leq x \leq 0.1 \\
S^{*} & :=0.008 \\
\rho_{0} & :=1.36835 \frac{\mathrm{kg}}{\mathrm{m}^{3}} \\
p_{0} & :=121277 \mathrm{~Pa} \\
\gamma & :=1.4
\end{aligned}
$$

Figure 9.2.12 on the following page shows the computational domain $\Omega$ together with the physical boundary conditions. The upper and lower sides $L_{+/-}$of the domain $\Omega$ are given by

$$
L_{+/-}:=\left(\begin{array}{c}
x \\
y(x) \\
z
\end{array}\right)
$$

with

$$
\begin{aligned}
x & \in[0 ; 0.1] \\
y(x) & = \pm \frac{1}{2} S(x) \quad \text { and } \\
z & \in[0 ; 0.01] .
\end{aligned}
$$

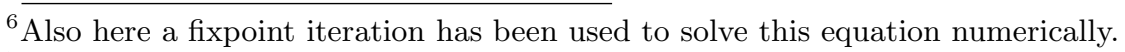

${ }^{7}$ The reference density $\rho_{0}$ and pressure $p_{0}$ are chosen such that the standard temperature and pressure (STP) values at the outflow boundary (see below) are obtained.
} 
The inflow and outflow values for the primitive variables density and pressure can be calculated using equations (9.2.4) to (9.2.7) with the corresponding (one-dimensional) cross-sections $S_{\text {in }}=S(0) \approx 0.017449$ and $S_{\text {out }}=S(0.1) \approx 0.010522$ :

$$
\begin{aligned}
\rho_{\text {in }} & =1.3170 \frac{\mathrm{kg}}{\mathrm{m}^{3}} \\
p_{\text {in }} & =114947 \mathrm{~Pa} \\
p_{\text {out }} & =101325 \mathrm{~Pa}
\end{aligned}
$$

The boundary treatment is handled as described in Section 4.7 on page 55 with the difference that the pressure at the inflow and outflow boundary is given, thus imposing a pressure gradient between inflow and outflow. Therefore, no velocity is prescribed at the boundaries.

The initial values are set to match the outflow values:

$$
\begin{aligned}
\rho_{0} & =1.2039 \frac{\mathrm{kg}}{\mathrm{m}^{3}} \\
v_{1,0} & =176.231 \frac{\mathrm{m}}{\mathrm{s}} \\
p_{0} & =101325 \mathrm{~Pa}
\end{aligned}
$$

The meshes that are used have $24 \times 10 \times 4$ elements up to $384 \times 160 \times 4$ elements (see Figure 9.2.11 on page 122 for the mesh with $48 \times 20 \times 4$ elements). The boundaries $P_{\text {front }}:=\{z=0\}$ and $P_{\text {rear }}:=\{z=0.01\}$ are treated as slip walls, therefore a quasi two-dimensional calculation is conducted: all variables are independent of the $z$ coordinate.

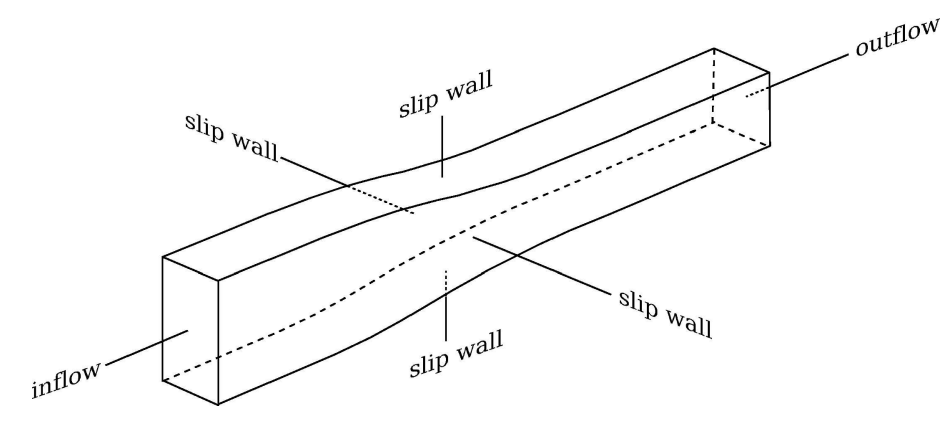

Figure 9.2.12: Nozzle flow: boundary conditions and domain $\Omega$ for the experimental setup.

\section{Results}

The pressure gradient that is imposed on the inflow and outflow boundaries, is the driving force for the flow. Therefore, the density is used for the study of the convergence behavior of the numerical solution. The density is only fixed at the inflow boundary. At the outflow boundary it is determined by the solution in the domain, which can be seen in the $x$ - $\rho$ diagram in Figure 9.2.13 on the next page. The density profiles on the different meshes also indicate an excellent convergence 
to the exact solution. This is confirmed by the history of the $L^{1}$-error of the density and the corresponding EOC evolution (that is displayed in Figure 9.2.14).

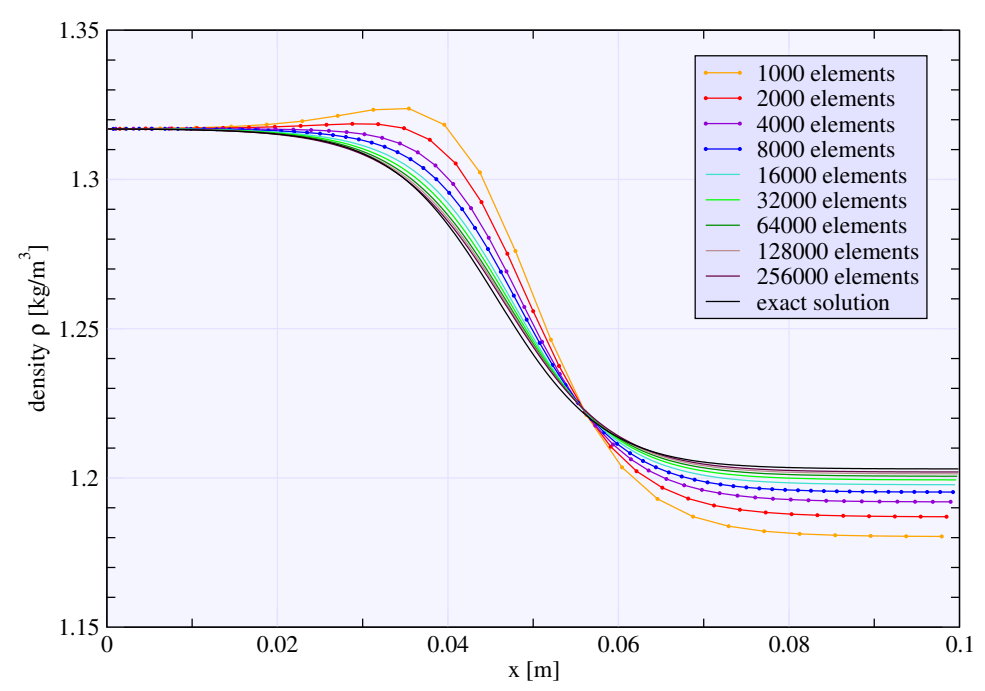

Figure 9.2.13: Nozzle flow: density profile along the nozzle on the different meshes and the exact density.
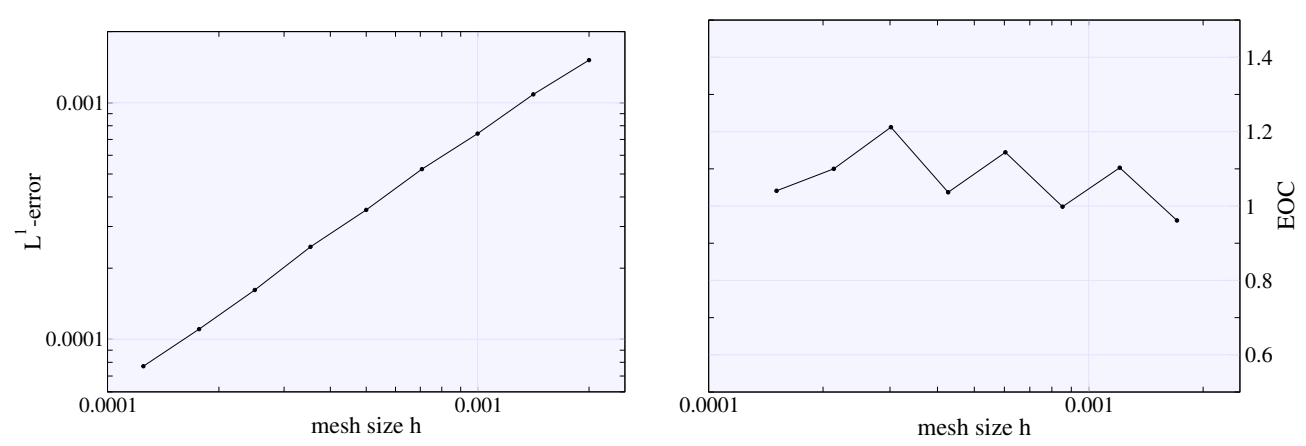

Figure 9.2.14: Nozzle flow: $L^{1}$-error of the density (left) and corresponding EOC (right) evolution on the different meshes.

\subsubsection{Channel with a Bump}

The last test case for inviscid flow is a truly two-dimensional problem. It is a stationary laminar flow in a channel with a bump (see Figure 9.2.15 on the next page). As the flow passes the narrow passage it is accelerated until a shock appears on the right-hand side of the bump. 


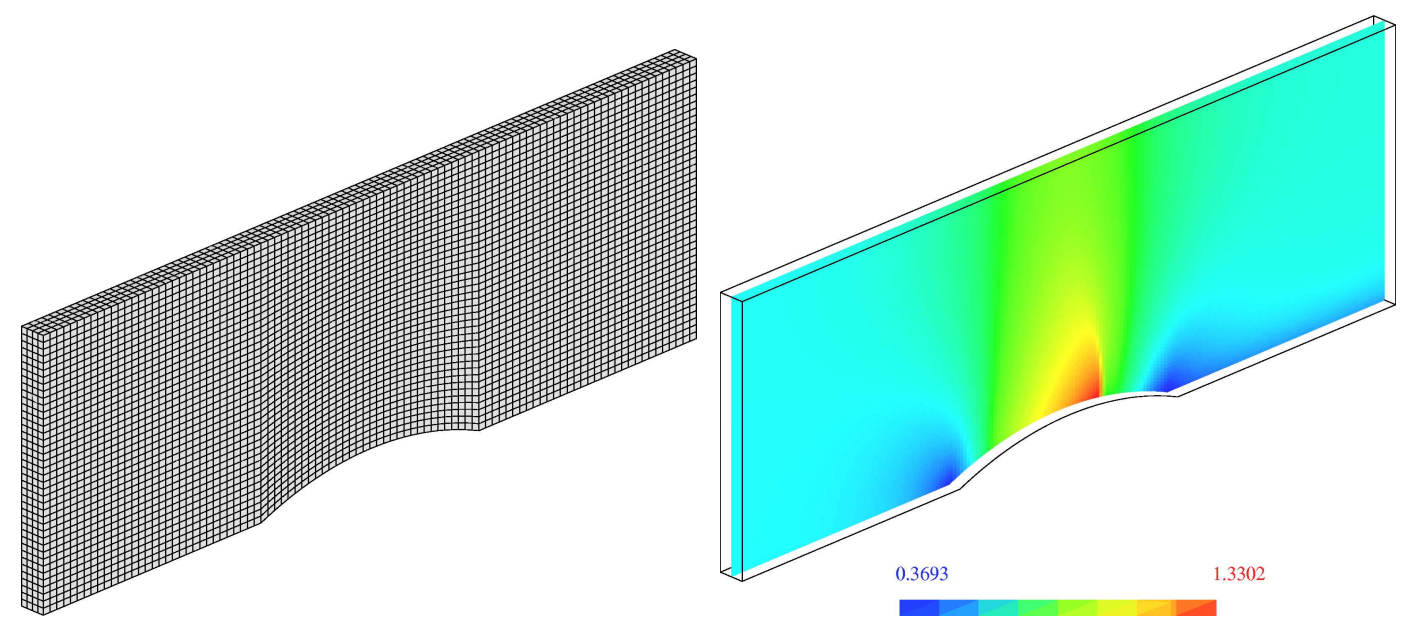

Figure 9.2.15: Channel with a bump: the $96 \times 40 \times 4$ element mesh (left) and a clipping plane of the computed Mach number on the $192 \times 80 \times 4$ element mesh (right).

\section{Validation of the Numerical Solution}

As there is, up to now, no method to obtain an exact solution of this problem, we have to rely on numerical solutions of other groups found in literature (e.g in [Hir92b]). Like in other literature, we consider a polytropic gas with adiabatic exponent $\gamma:=1.4$.

\section{Setup of the Numerical Experiment}

The setup consists of the computational domain $\Omega$ and the physical boundary conditions as depicted in Figure 9.2.16 on the facing page. The domain is derived from a channel $\bar{\Omega}:=[0 ; 0.6] \times[0 ; 0.2] \times[0 ; 0.02]$ where a cylindrical bump of cord length $l:=0.2$ and height $h:=0.02$ is cut out in the middle of the lower plane. The axis of this cylinder is therefore $M=(0.3,-0.24)^{T} \times[0 ; 0.02]$ and its radius $R=0.26$. Thus, the position of the lower side $L$ is given by

$$
L=\left(\begin{array}{c}
x \\
y(x) \\
z
\end{array}\right)
$$

with

$$
\begin{array}{rll}
x & \in[0 ; 0.6], \\
y(x) & = \begin{cases}R(\sin (\alpha)-1)+0.02 & \text { for } 0.2 \leq x \leq 0.4 \\
0 & \text { otherwise }\end{cases} \\
\cos (\alpha) & =\frac{x-0.3}{R} \text { and } \\
z & \in[0 ; 0.02] .
\end{array}
$$

The numerical inflow and outflow boundary conditions are again handled according to Section 4.7 on page 55 with the boundary values identical to the initial 
values. These initial values in primitive variables are fixed as:

$$
\begin{aligned}
\rho_{0} & :=1.2039 \frac{\mathrm{kg}}{\mathrm{m}^{3}} \\
v_{0} & :=231.7411 \frac{\mathrm{m}}{\mathrm{s}} \\
p_{0} & :=101325 \mathrm{~Pa}
\end{aligned}
$$

resulting in a Mach number of $M=0.675$.

The used meshes have a resolution of $24 \times 10 \times 4$ elements up to $384 \times 160 \times 4$ elements. The boundaries $P_{\text {front }}:=\{z=0\}$ and $P_{\text {rear }}:=\{z=0.02\}$ are treated, as in the nozzle flow, as slip walls, therefore again a quasi two-dimensional calculation is conducted.

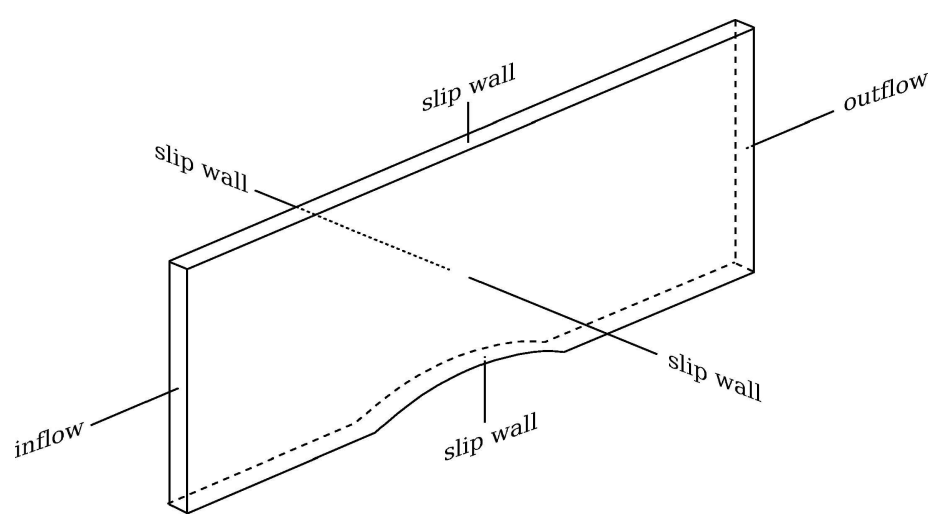

Figure 9.2.16: Channel with a bump: boundary conditions and domain $\Omega$ for the experimental setup.

\section{Results}

In Figure 9.2.17 on the following page the Mach number along the bottom side of the channel (the oscillating line) and on the horizontal plane $P_{\text {middle }}:=\{y=0.1\}$ (the smooth line) is displayed, on top our results, and below the solution from literature (as given in [Hir92b]). As can be seen, the convergence of our numerical solutions to the reference solution is very good as the mesh size decreases. On the numerical solutions obtained on the finer meshes, all the features of the literature solution are reproduced exactly: the two sharp minima right at the front and tail of the bump, the supersonic region terminated by a shock, and the little kink right after the shock.

In the next Figure 9.2.18 on the next page the iso-Mach contours of our solution on the finest mesh of $384 \times 160 \times 4$ elements are compared to the literature on the whole domain. And also here, no difference is discernable. 

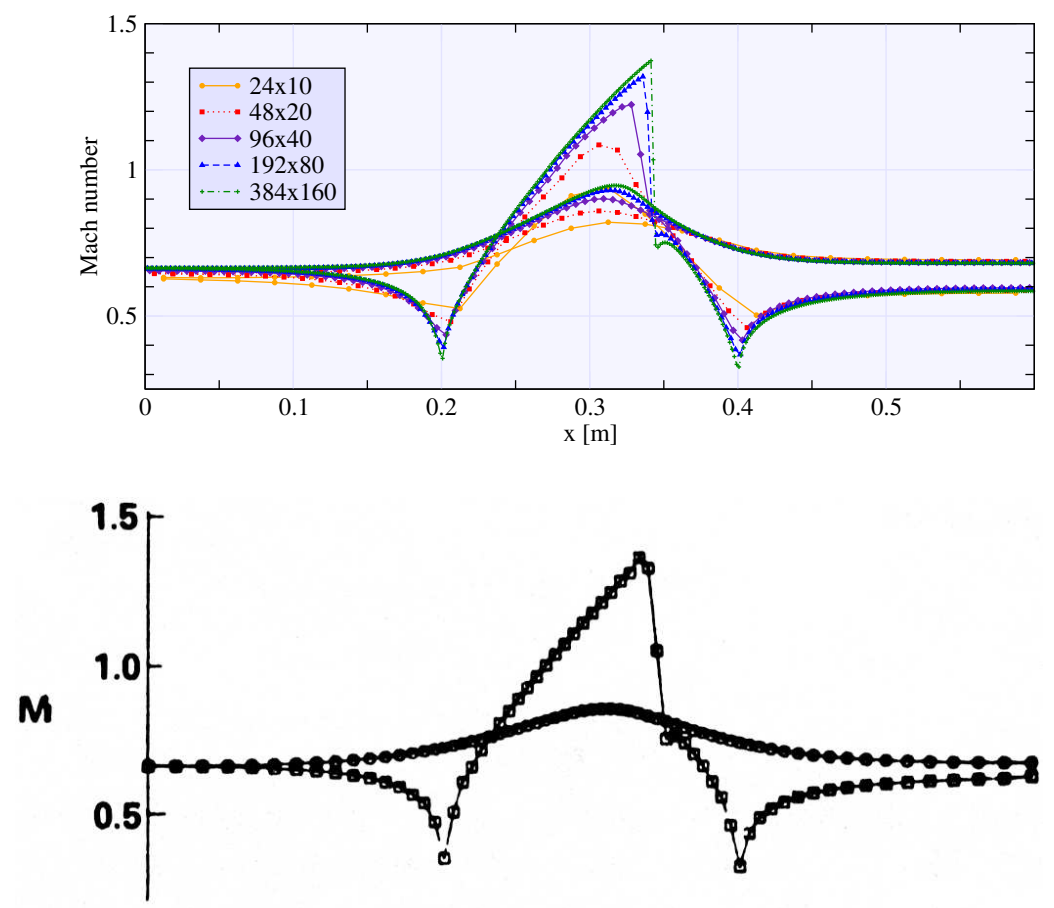

Figure 9.2.17: Channel with a bump: comparison of our results (top) with the literature (below) (shown are the Mach numbers along the bottom plane and the middle plane).

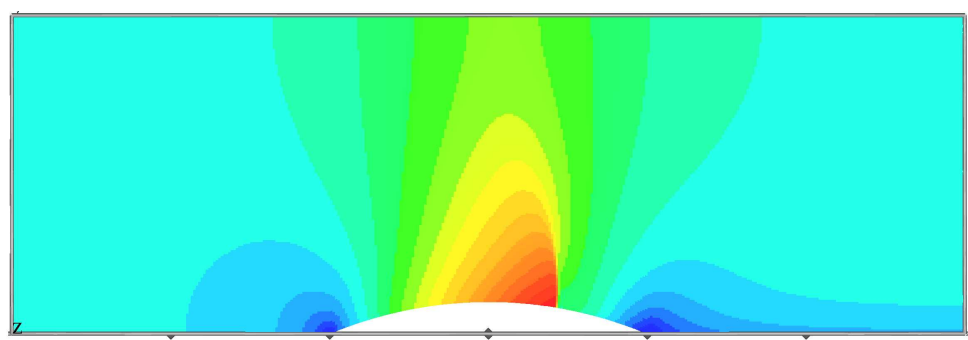

$\begin{array}{llllll}0.40 & 0.60 & 0.80 & 1.00 & 1.20 & 1.40\end{array}$

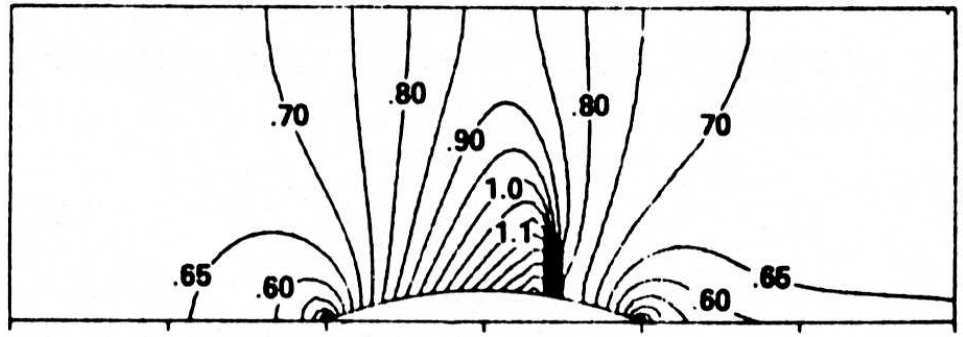

Figure 9.2.18: Channel with a bump: comparison of our results on the $384 \times 160 \times 4$ element mesh (top) with the literature (below) (shown are the Mach number contours). 


\subsection{Viscous Flow}

The next four problems in this section test the viscous part of the software. This time the viscosity and thermal conductivity are also taken into account. For large Reynolds numbers the viscosity is very small, such that a fluid with this Reynolds number can be treated as an inviscid fluid in most cases (we will see an example in the last test problem 9.3.4 on page 141 of this section). This is certainly not true in the close vicinity of walls where a boundary layer is formed for viscous fluids (see the Blasius plate problem 9.3.3 on page 135). The mathematical model of this viscous flow is governed by the compressible Navier-Stokes equations, as given in Section 3.3.2 on page 32. The numerical scheme is described in Sections 4.3 on page $47,4.7,4.4$, and 4.8 , with the viscous flux from Section 4.5 on page 52 . Also in this section, all the values from the diagrams are given in the tables in Appendix A on page 205.

\subsubsection{Poiseuille Flow}

The first test case in this category is the classical two-dimensional stationary laminar duct flow or Poiseuille flow (see Figure 9.3.1). A fluid flows through a channel with fixed upper and lower walls of height $h=2 a$. Right at the walls the boundary condition forces the flow velocity to zero. And the viscosity of the fluid transports this friction towards the middle of the channel. This physical setup is shown in Figure 9.3.2 on page 131.

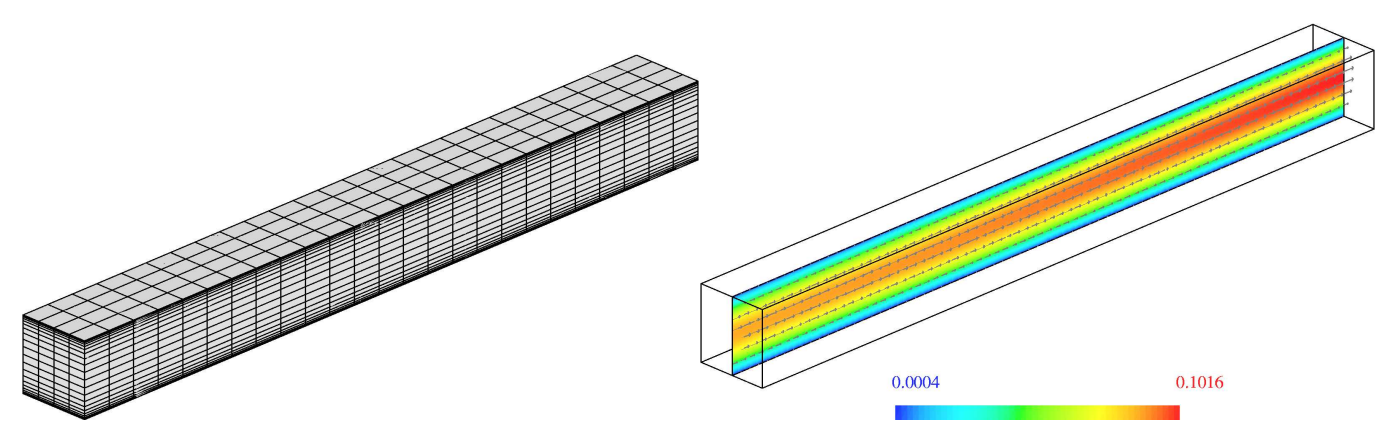

Figure 9.3.1: Poiseuille flow: the $25 \times 20 \times 4$ element mesh (left) and a clipping plane of the computed Mach number with velocity vectors on the $100 \times 80 \times 4$ element mesh (right) (the images are scaled with factor 50 in $y$-direction and 5 in $z$-direction).

\section{Exact Solution}

In the incompressible case an exact solution can be calculated (see e.g. [Tru89]). Let the boundary condition for the velocity $v=\left(v_{1}, v_{2}\right)^{T}$ at the upper and lower boundary be given by $v_{1}(x, y)=v_{2}(x, y)=0$ for $y=a$ and $y=-a$. Then the absence of a velocity in $y$-direction yields for the viscous fluid in the velocity distribution

$$
v(x, y)=-\frac{1}{2 \eta}\left(a^{2}-y^{2}\right) \frac{d p}{d x},
$$


i.e. the velocity $v$ is independent of $x$. The pressure is independent of $y$ and the pressure gradient is constant along the duct:

$$
\frac{d p}{d x}=\text { const }
$$

The Poiseuille flow is therefore also called pressure induced flow through a duct with a parabolic velocity distribution.

\section{Setup of the Numerical Experiment}

The viscosity $\eta$, the thermal conductivity $\lambda$, and the specific heat capacity at constant pressure $c_{p}$ are set to the values of air at standard temperature and pressure:

$$
\begin{aligned}
\eta & :=1.834 \cdot 10^{-5} \frac{\mathrm{Ns}}{\mathrm{m}^{2}} \\
\lambda & :=2.540 \cdot 10^{-2} \frac{\mathrm{N}}{\mathrm{s} \mathrm{K}} \\
c_{p} & :=1004.8 \frac{\mathrm{m}^{2}}{\mathrm{~s}^{2} \mathrm{~K}}
\end{aligned}
$$

The adiabatic exponent $\gamma$ is fixed, as in all the following test problems, at:

$$
\gamma:=1.4
$$

resulting in a specific gas constant $R$ of air of:

$$
R:=287.10 \frac{\mathrm{J}}{\mathrm{kg} \mathrm{K}}
$$

The size of the computational domain (as shown in Figure 9.3.2 on the facing page) is chosen as

$$
\Omega:=[0 ; 0.1] \times[0 ; 0.0002] \times[0 ; 0.002]
$$

and it follows for $a$ :

$$
a=0.0001 \mathrm{~m}
$$

The treatment of the fixed wall boundaries is described in Section 4.7 on page 55 . The inflow and outflow boundary values are fixed such that the pressure gradient $\frac{\Delta p}{\Delta x}$ induces a maximum velocity of $10 \%$ of the speed of sound. Therefore, the flow behavior is almost incompressible, which is important because the exact solution is only valid for an incompressible flow. So the boundary pressures are prescribed as:

$$
\begin{aligned}
p_{\text {in }} & =114083.6 \mathrm{~Pa} \\
p_{\text {out }} & =101325 \mathrm{~Pa}
\end{aligned}
$$

The density $\rho_{\text {in }}$ is set to the standard atmospheric value:

$$
\rho_{\text {in }}=1.2039 \frac{\mathrm{kg}}{\mathrm{m}^{3}}
$$

$\rho_{\text {out }}$ is treated as numerical boundary condition, and also for the velocities $v_{\text {in }}$ and $v_{\text {out }}$ no value can be set in order to not disturb the formation of the parabolic 
distribution of the velocity. The front and rear boundaries $P_{\text {front }}=\{z=0\}$ and $P_{\text {rear }}=\{z=0.002\}$ are again treated as slip wall boundaries as to conduct a quasi two-dimensional calculation. All variables are independent of the $z$ coordinate and the velocity in $z$-direction is set to 0 .

As initial values the exact solution was used. This is done in order to speed up the convergence to a stationary state. This stationary state is not influenced by the choice of initial values (see also Section 6.3 on page 97 ).

The used meshes contain from $12 \times 10 \times 4$ elements up to $200 \times 160 \times 4$ elements (see Figure 9.3 .1 on page 129 for the $25 \times 20 \times 4$ element mesh).

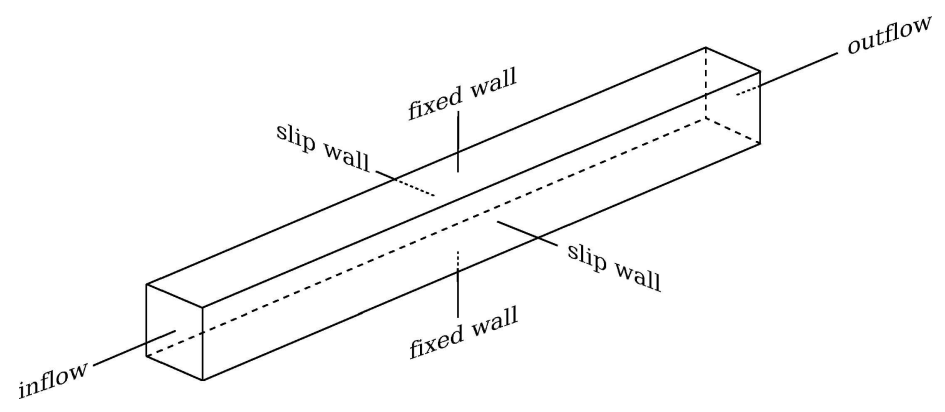

Figure 9.3.2: Poiseuille flow: boundary conditions and domain $\Omega$ for the experimental setup.

\section{Results}

The Poiseuille flow is a stationary problem. Because our numerical scheme uses a time-stepping algorithm, we calculate a time-dependent problem with the asymptotical stationary solution. Thus, in the first place we need to check the temporal convergence to a stationary state. This can be seen in Figure 9.3.3 on the following page, e.g. for the $50 \times 40 \times 4$ element mesh. After a steady state is reached, the solutions on the different meshes can be compared. This is done in Figure 9.3.4 for the velocity profile. It is shown that already on the coarsest mesh the calculated velocity approximates the exact one quite well. On the finer meshes the accuracy is greatly enhanced. This is verified on the $L^{1}$-error plot of the velocity and the associated EOC history, as displayed in Figure 9.3.5 on the next page. The shown mesh size $h$ in these two diagrams is the length of the smallest edge, that is located in $y$-direction in the elements at the upper and lower boundary (cf. also the mesh depicted on the left-hand side of Figure 9.3.1 on page 129).

Remark 9.3.1. The numerical viscosity of the convective flux does not affect the solution of the Poiseuille flow in this configuration because it only acts along the flow direction. Here the solution is determined by the viscosity of the fluid normal to the flow. 


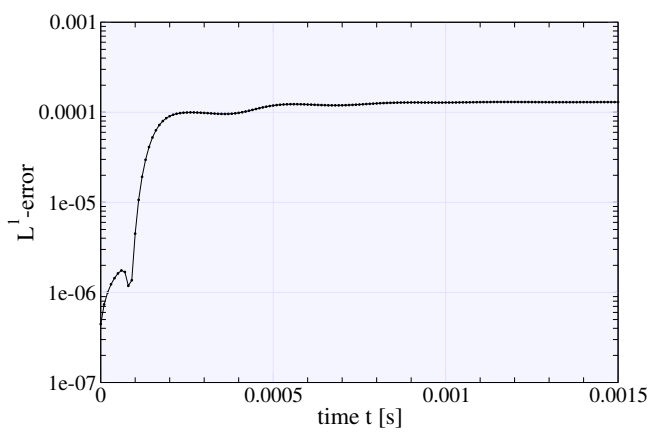

Figure 9.3.3: Poiseuille flow: temporal convergence of the time-dependent problem to a stationary state on the $50 \times 40 \times 4$ element mesh (the $L^{1}$-error of the velocity $v_{1}$ is shown).

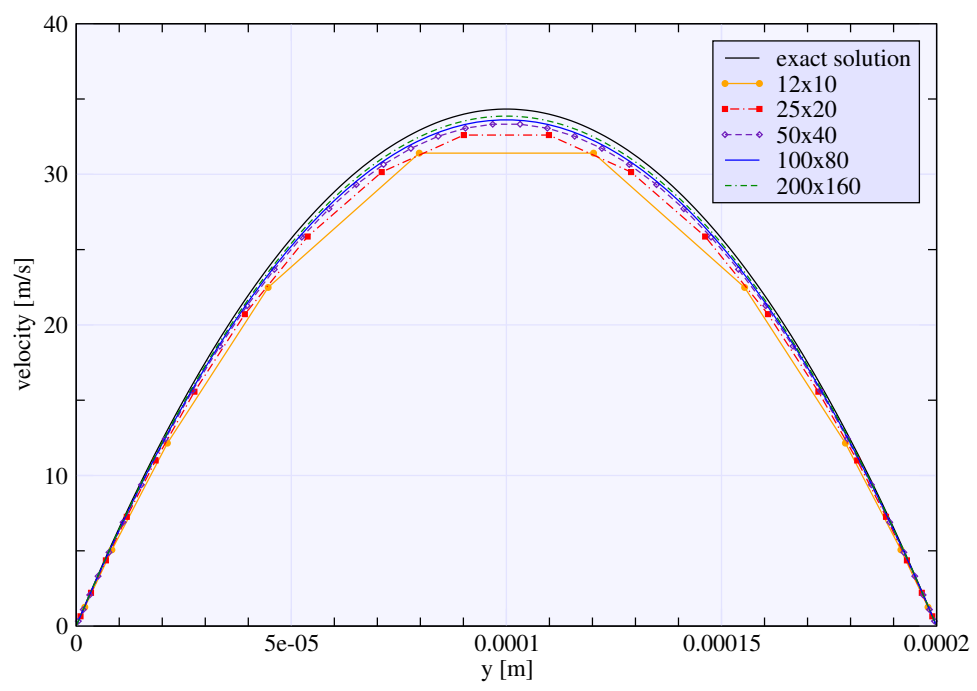

Figure 9.3.4: Poiseuille flow: velocity profile on the different meshes and exact velocity.
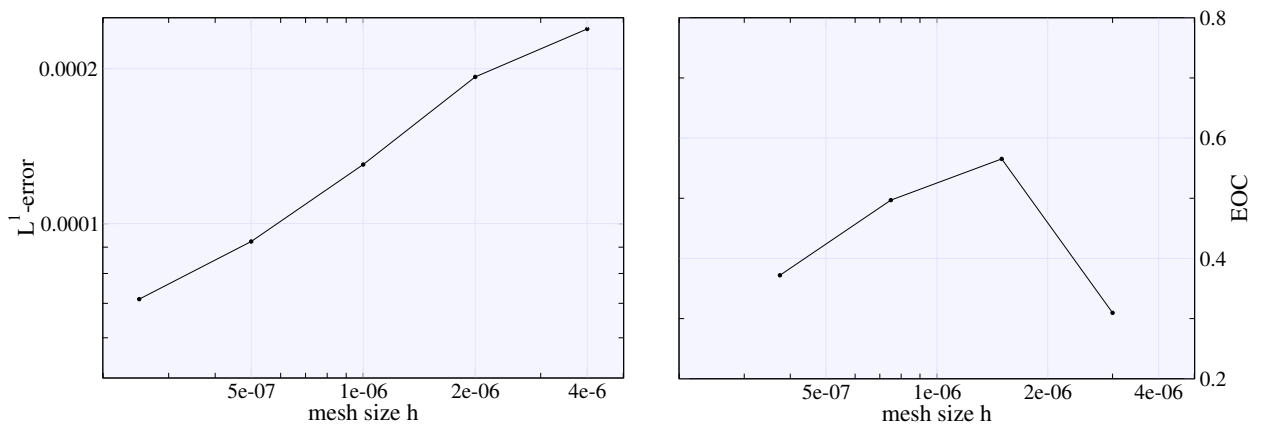

Figure 9.3.5: Poiseuille flow: $L^{1}$-error convergence history of the velocity on the different meshes (left) and the associated EOC plot (right). 


\subsubsection{Couette Flow}

Very similar to the just described Poiseuille flow is the laminar two-dimensional stationary shear flow or Couette flow (see Figure 9.3.6). Unlike in the Poiseuille flow, where a pressure gradient was the driving force of the flow, in the Couette flow a moving upper wall sets the viscous fluid in motion. The physical setup is similar to the one described in the previous Paragraph 9.3.1 on page 129 and can be seen in Figure 9.3.7 on the next page. The two plates are situated at a distance $h$. The upper one $P_{\text {upper }}=\{y=h\}$ is moving with a constant speed $u_{\text {plate }}$ whereas the lower one is fixed.

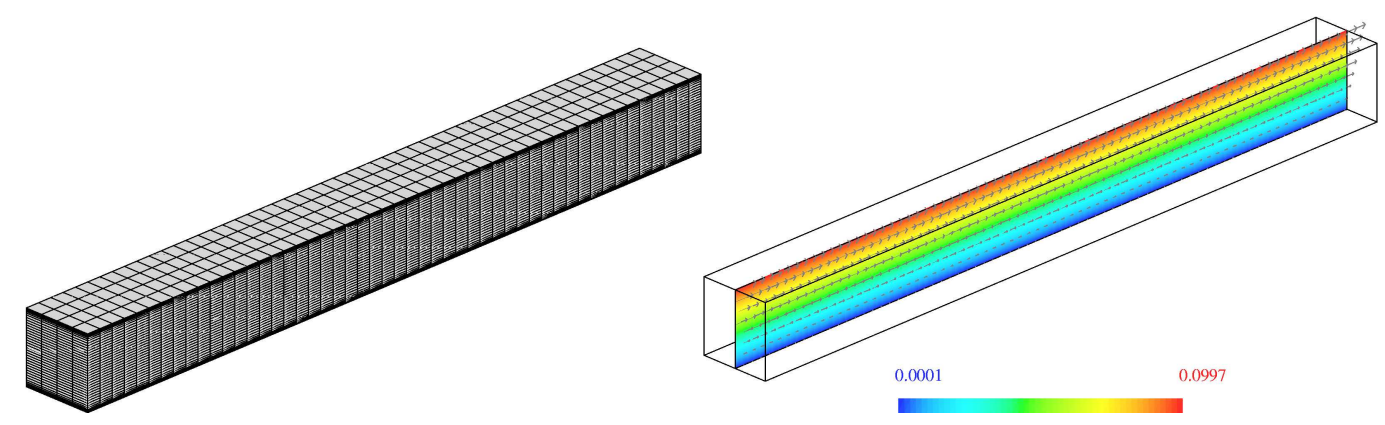

Figure 9.3.6: Couette flow: the $50 \times 40 \times 4$ element mesh (left) and a clipping plane of the computed Mach number with velocity vectors on the $100 \times 80 \times 4$ element mesh (right) (the images are scaled by a factor 50 in $y$-direction and 5 in $z$-direction).

\section{Exact Solution}

As in the Poiseuille flow, an exact solution can be given for the Couette flow in the case of an incompressible fluid (cf. [Tru89]). Let the boundary conditions for the velocity $v=\left(v_{1}, v_{2}\right)^{T}$ of the flow at the upper and lower boundary be given by $v_{1}(x, 0):=v_{2}(x, 0):=0$ and $v_{1}(x, h):=u_{\text {plate }}, v_{2}(x, h):=0$. Then it can be shown for $\frac{d p}{d x}=0$ :

$$
\begin{aligned}
v_{1}(x, y) & =\frac{y}{h} u_{\text {plate }} \\
v_{2}(x, y) & =0
\end{aligned}
$$

\section{Setup of the Numerical Experiment}

The same values for the viscosity $\eta$, the thermal conductivity $\lambda$, the specific heat capacity at constant pressure $c_{p}$, and the adiabatic exponent $\gamma$ are used as in the 
Poiseuille flow:

$$
\begin{aligned}
\eta & :=1.834 \cdot 10^{-5} \frac{\mathrm{Ns}}{\mathrm{m}^{2}} \\
\lambda & :=2.540 \cdot 10^{-2} \frac{\mathrm{N}}{\mathrm{s} \mathrm{K}} \\
c_{p} & :=1004.8 \frac{\mathrm{m}^{2}}{\mathrm{~s}^{2} \mathrm{~K}} \\
\gamma & :=1.4
\end{aligned}
$$

Also the domain $\Omega$ is identical:

$$
\Omega:=[0 ; 0.1] \times[0 ; 0.0002] \times[0 ; 0.002] .
$$

Therefore, we have $h=0.0002$ for the distance between the upper and the lower plate. The velocity $u_{\text {plate }}$ of the upper plate is set to

$$
u_{\text {plate }}:=34.332 \frac{\mathrm{m}}{\mathrm{s}}
$$

being $10 \%$ of the speed of sound $c$.

The boundary conditions (shown in Figure 9.3.7) are treated in the same way as above, with the exception of a zero pressure gradient at the inflow and outflow boundary:

$$
\begin{aligned}
p_{\text {in }}:=p_{\text {out }} & :=101325 \mathrm{~Pa} \\
\rho_{\text {in }} & :=1.2039 \frac{\mathrm{kg}}{\mathrm{m}^{3}}
\end{aligned}
$$

Thus, no velocity is prescribed at the inflow and outflow boundaries.

Also, the setting of the initial values and the meshes are the same as in the previous test case 9.3.1 on page 129 .

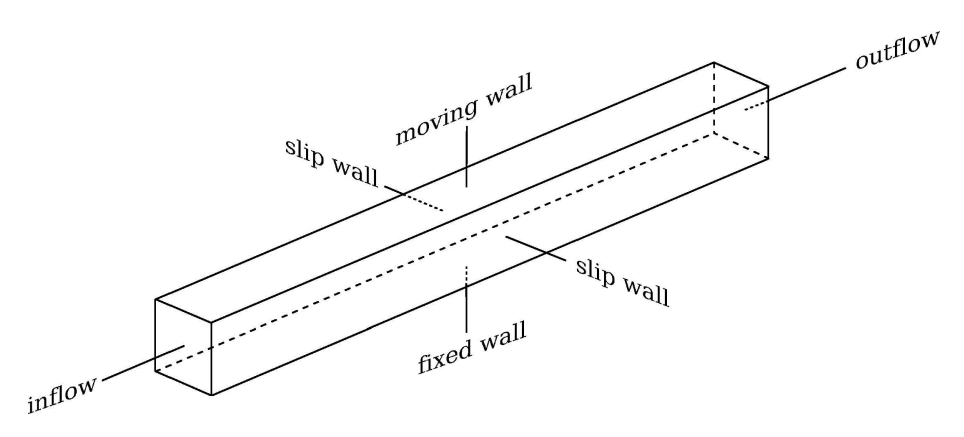

Figure 9.3.7: Couette flow: boundary conditions and domain $\Omega$ for the experimental setup.

\section{Results}

Just as the Poiseuille flow, the Couette flow is a stationary problem. Therefore, we need to check for the temporal convergence to a steady state. This can be seen in 
Figure 9.3 .8 for the $50 \times 40 \times 4$ element mesh. After approximately $0.0015 \mathrm{~s}$ (which needs some 870.000 steps in the case of this mesh) the $L^{1}$-error of the velocity does not change significantly any more, and a stationary state is assumed to be reached. This is verified on all meshes. Now, we can compare the results on the different meshes to the exact solution. On the plots in Figure 9.3.9, one can see the excellent approximation of the numerical solution even on the coarsest mesh. In order to enhance the visibility of the differences, a detailed view is shown on the right-hand side of Figure 9.3.9. Also the convergence to the exact solution is very good, which can be seen in Figure 9.3.10 on the next page. The $L^{1}$-error decreases with reducing mesh size $h$ and the corresponding EOC values are around 0.8 to 1 .

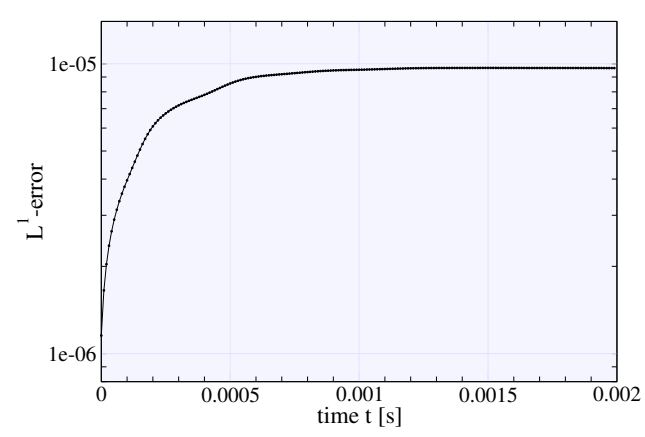

Figure 9.3.8: Couette flow: temporal convergence of the time-dependent problem to a stationary state on the $50 \times 40 \times 4$ element mesh (shown is the $L^{1}$-error of the velocity $v_{1}$ ).
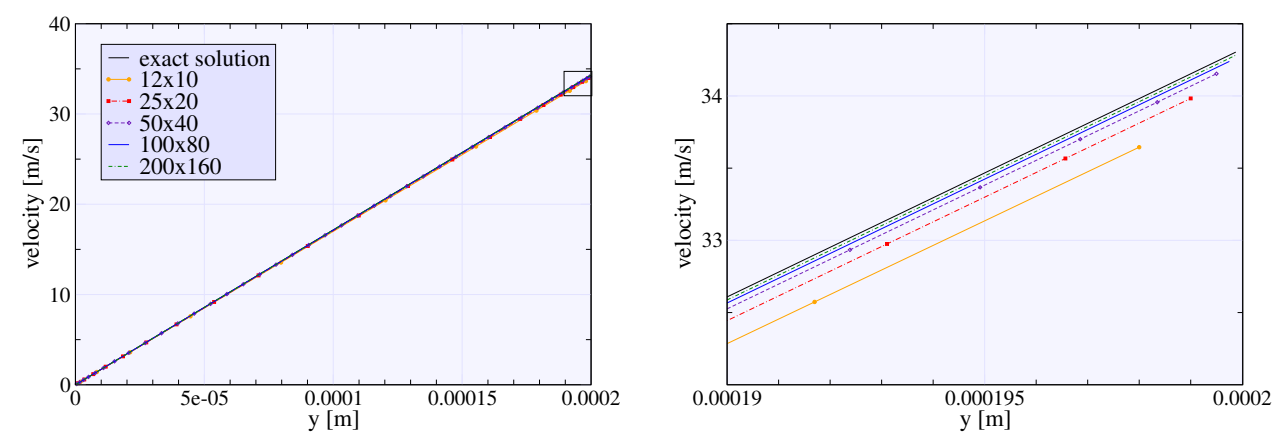

Figure 9.3.9: Couette flow: velocity profile on the different meshes and exact velocity (left) and a detailed view of the upper part of the plot (right).

\subsubsection{Blasius Plate}

In this test problem a laminar stationary boundary layer flow is studied (see Figure 9.3.11 on the following page). A fluid flows over a very thin plate and develops a boundary layer of increasing thickness with distance to the front edge of the plate. 

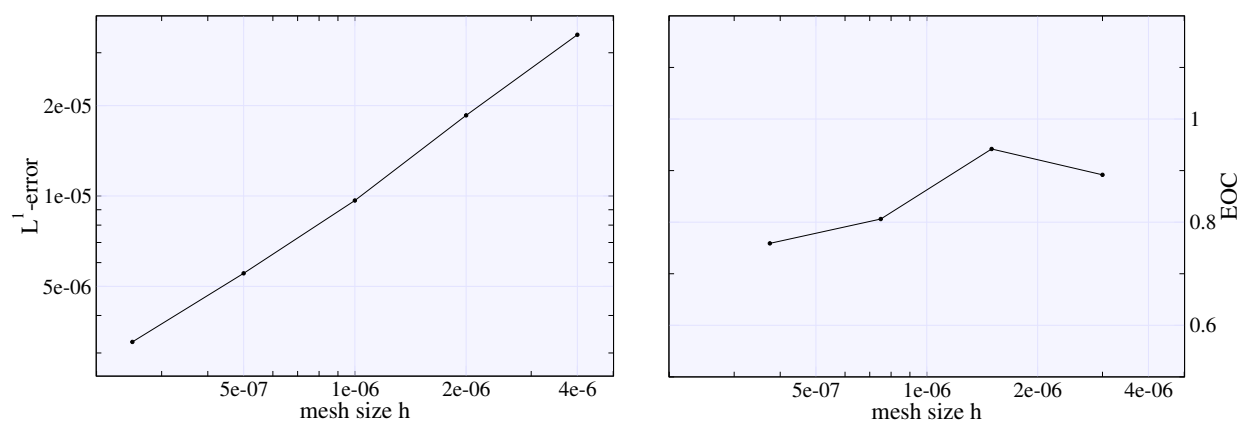

Figure 9.3.10: Couette flow: $L^{1}$-error convergence of the velocity on the different meshes (left) and the associated EOC values (right).

The plate begins at $x=0$, is parallel to the $x$-axis and semi-infinitely long. The approach velocity of the flow $v_{\infty}$ is constant and parallel to the $x$-axis. Therefore, no pressure gradient in $x$-direction is present in the flow: $\frac{d p}{d x}=0$. This Blasius plate problem is sketched in Figure 9.3.14 on page 139.

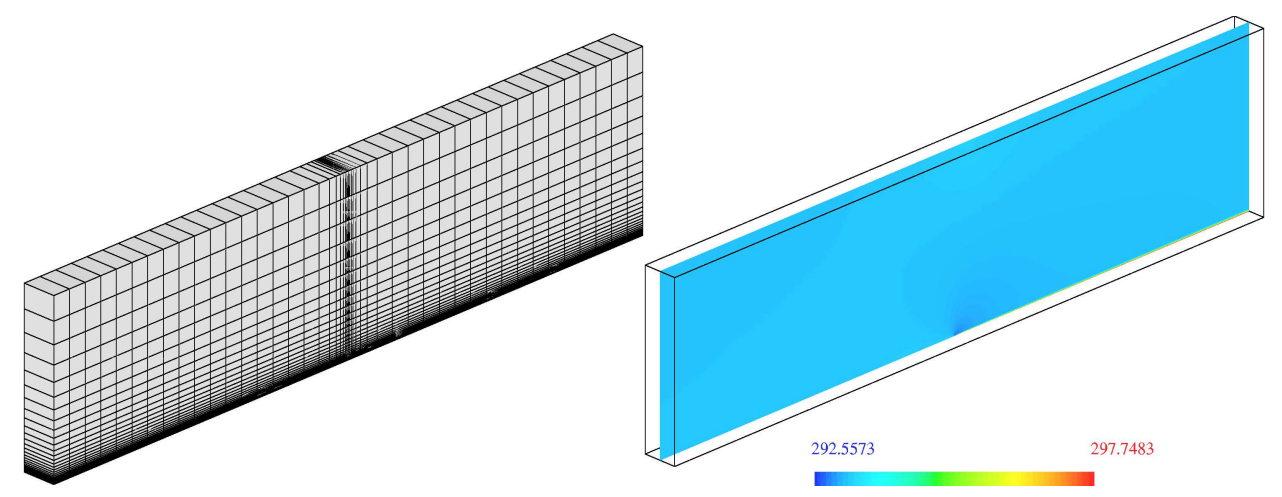

Figure 9.3.11: Blasius plate: the $50 \times 30 \times 1$ element mesh (left) and a clipping plane of the computed temperature on the $100 \times 80 \times 1$ element mesh (right).

\section{Exact Solution}

For the incompressible case and under the above-stated assumptions the NavierStoke equations can be transformed into the PRANDTL boundary layer equations (cf. e.g. [SG97, LL95]):

$$
\begin{aligned}
v_{1} \frac{\partial v_{1}}{\partial x}+v_{2} \frac{\partial v_{1}}{\partial y} & =\eta \frac{\partial^{2} v_{1}}{\partial y^{2}} \\
\frac{\partial v_{1}}{\partial x}+\frac{\partial v_{2}}{\partial y} & =0
\end{aligned}
$$


with the boundary conditions:

$$
\begin{aligned}
y=0: & u=0, v=0 \\
y \rightarrow \infty: & u=v_{\infty}
\end{aligned}
$$

In order to solve these equations, a self-similar solution is assumed. Therefore, a new similarity variable

$$
\xi:=y \sqrt{\frac{v_{\infty}}{2 \eta x}}
$$

is defined. After introducing a dimensionless stream function $f(\xi)$ one obtains for the velocity components

$$
\begin{aligned}
v_{1} & =v_{\infty} f^{\prime}(\xi) \\
v_{2} & =\sqrt{\frac{\eta v_{\infty}}{2 x}}\left(\xi f^{\prime}-f\right)
\end{aligned}
$$

and it follows for the stream function $f(\xi)$ the ordinary differential equation

$$
f^{\prime \prime \prime}+f f^{\prime \prime}=0,
$$

which is also called Blasius equation. The boundary conditions translate into

$$
\begin{aligned}
\xi=0: & f=0, f^{\prime}=0, \\
\xi \rightarrow \infty: & f^{\prime}=1 .
\end{aligned}
$$

This boundary value problem for the ODE can be solved by a Runge-Kutta method with the "shooting approach" in order to guess an initial value for $f^{\prime \prime}(0)$. This value has been computed to be $f^{\prime \prime}(0)=0.4696$. The solution for $f, f^{\prime}$, and $f^{\prime \prime}$ is displayed in Figure 9.3.12 on the following page. With the help of this solution the exact profile of the velocity components (also over the similarity variable $\xi$ ) can be given (see Figure 9.3.13 on the next page).

For the temperature distribution within the boundary layer (cf. [SG97, Section 9.6]) we assume an adiabatic wall, i.e. no heat flux is present. With the additional assumption of a Prandtl number of $\operatorname{Pr}:=1$, an exact temperature profile can be given with the help of the above defined stream function $f(\xi)$ :

$$
T(\xi)=\frac{v_{\infty}^{2}}{2 c_{p}}\left(1-\left(f^{\prime}(\xi)\right)^{2}\right)+T_{\infty}
$$

Here $T_{\infty}$ is the temperature of the approaching fluid. This exact temperature is also shown in Figure 9.3.13 on the following page.

These theoretically derived exact solutions have been reproduced very accurately by physical experiments (see [SG97]). 

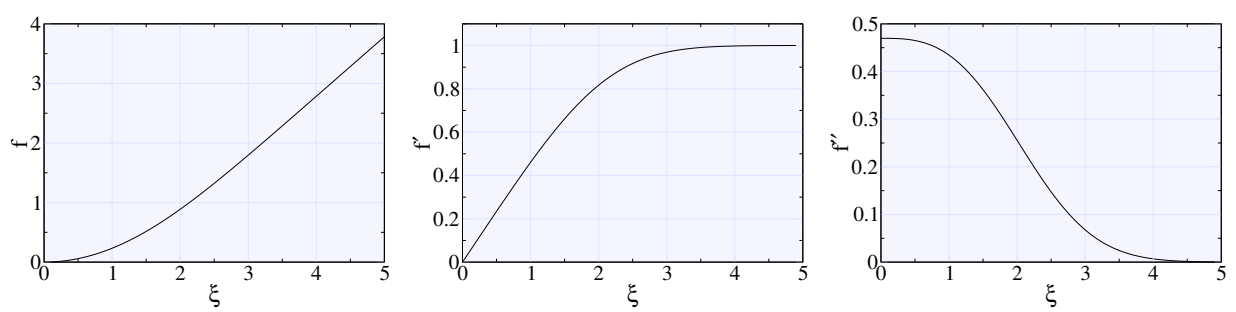

Figure 9.3.12: Blasius plate: the stream function $f$ and its derivatives.
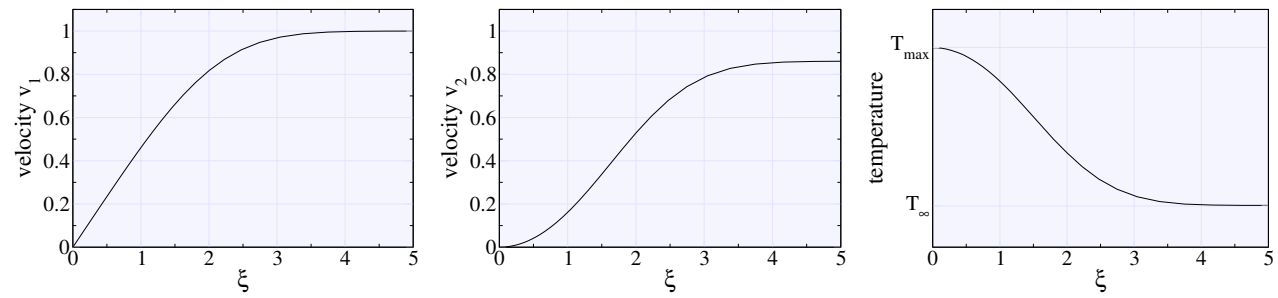

Figure 9.3.13: Blasius plate: exact velocity profiles $v_{1}$ and $v_{2}$ (left and middle) and exact temperature profile (right) (the similarity variable $\xi:=y \sqrt{\frac{v_{\infty}}{2 \eta x}}$ gives the distance to the plate in $y$-direction for fixed position $x$ ).

\section{Setup of the Numerical Experiment}

The viscosity $\eta$ is set to the viscosity of air at standard temperature and pressure:

$$
\eta:=1.834 \cdot 10^{-5} \frac{\mathrm{N} \mathrm{s}}{\mathrm{m}^{2}}
$$

The Prandtl number of $\operatorname{Pr}:=1$ (see above) forces the thermal conductivity $\lambda$ to be

$$
\lambda=\frac{\eta c_{p}}{\operatorname{Pr}}=1.843 \cdot 10^{-2} \frac{\mathrm{N}}{\mathrm{s} \mathrm{K}}
$$

(as the Prandtl number of air is $\operatorname{Pr}=0.726$, this value for $\lambda$ comes quite close to the real thermal conductivity of air at standard temperature and pressure of $\left.\lambda=2.540 \cdot 10^{-2} \frac{\mathrm{N}}{\mathrm{sK}}\right)$. The specific heat capacity at constant pressure $c_{p}$ at this temperature is equal to:

$$
c_{p}:=1004.8 \frac{\mathrm{m}^{2}}{\mathrm{~s}^{2} \mathrm{~K}}
$$

The adiabatic exponent $\gamma$ is fixed at:

$$
\gamma:=1.4
$$

The self-similarity solution is not valid at the front of the plate (at $x=0$ ). In order to diminish this disturbing influence, the error is calculated at the position $x=0.05$ and the computing domain is given by

$$
\Omega:=[-0.1 ; 0.1] \times[0 ; 0.05] \times[0 ; 0.01] .
$$


The value for the velocity of the approaching fluid $v_{\infty}$ has to be set such that the flow can be regarded as incompressible (for the exact solution to be valid). Therefore, a velocity of

$$
v_{\infty}:=100 \frac{\mathrm{m}}{\mathrm{s}}
$$

is chosen, also to have a measurable temperature boundary layer (cf. equation (9.3.3)).

The boundary conditions are shown in Figure 9.3.14). As inflow values the density $\rho_{\text {in }}$ and the pressure $p_{i n}$ are set to

$$
\begin{aligned}
& \rho_{\text {in }}=1.2039 \frac{\mathrm{kg}}{\mathrm{m}^{3}}, \\
& p_{\text {in }}:=101325 \mathrm{~Pa},
\end{aligned}
$$

and at the outflow boundary the exact values for the velocities $v_{1}$ and $v_{2}$ and for the temperature $T$, given in equations (9.3.1), (9.3.2), and (9.3.3), are prescribed. A detailed description of the boundary treatment is given in Section 4.7 on page 55 .

As initial values, the data for the ambient flow with the parameters

$$
\begin{aligned}
\rho(\mathbf{x}, 0) & :=1.2039 \frac{\mathrm{kg}}{\mathrm{m}^{3}} \\
v_{1}(\mathbf{x}, 0) & :=v_{\infty}, \\
v_{2}(\mathbf{x}, 0) & :=0 \frac{\mathrm{m}}{\mathrm{s}} \\
p(\mathbf{x}, 0) & :=101325 \mathrm{~Pa},
\end{aligned}
$$

for $\mathbf{x} \in \Omega$ is used.

The meshes are composed of $26 \times 15 \times 1$ elements up to $400 \times 240 \times 1$ and are highly refined towards the boundary layer up to an edge ratio of $1: 60$, resulting in 3 to 36 elements within the boundary layer (see Figure 9.3.11 on page 136 for the $50 \times 30 \times 1$ elements mesh).

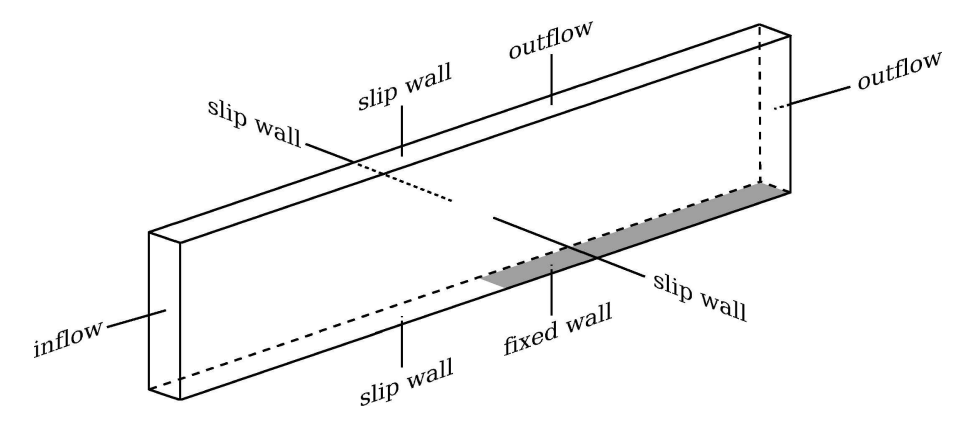

Figure 9.3.14: Blasius plate: boundary conditions and domain $\Omega$ for the experimental setup.

\section{Results}

The Blasius plate, as the Poiseuille (Paragraph 9.3.1 on page 129) and Couette flow (Paragraph 9.3.2 on page 133), is also a stationary problem. Therefore, we 
compute the numerical solution with our time-stepping method until a steady state is reached. As can be seen in Figure 9.3.15 for the $50 \times 30 \times 1$ element mesh this temporal convergence towards a stationary state for the velocities $v_{1}$ and $v_{2}$, and for the temperature $T$ is finished after about $0.01 \mathrm{~s}$ (being the equivalent of almost 90000 steps on this mesh). The $L^{1}$-error in these graphs, as well as in the following ones, is measured near the plate in the interval $0 \leq \xi \leq 5$.

Now, we can study the quality of the numerical solution with respect to the exact solution. The approximation of the velocity $v_{1}$ (shown on the left-hand side of Figure 9.3.16 on the next page) is already good for the coarse meshes, and on the finer meshes it is hard to distinguish the difference between numerical and exact solution. This can not be said for the velocity $v_{2}$. The calculated solution on the coarsest mesh is quite close to the exact one but then a convergence can only be seen near the plate for $\xi \leq 1.5$. For larger $\xi$, the approximation on the finer meshes tends to be farther away from the exact solution. This can be explained by the difficult boundary treatment of the upper boundary, which is actually an outflow boundary where the exact value of the vertical velocity $v_{2}$ cannot be set on the whole interval $0 \leq x \leq 0.1$. This results from the fact that the exact solution is not valid at the front edge of the plate. For $x \rightarrow 0$ one obtains $v_{2} \rightarrow \infty$. Thus, the front edge of the plate has a significant impact on the vertical velocity $v_{2}$, even far away from the edge. A second problem might be the compressibility of our fluid. The assumptions of constant density $\rho$ and constant pressure $p$ orthogonal to the plate are not exactly satisfied by our numerical setup (see also [Sch97], where this problem for the vertical velocity is also encountered, and as well in [Fuj95] only the horizontal velocity is studied, no reference to the vertical velocity is made). But, again, for the temperature $T$ (shown in Figure 9.3.16 (right)) a very good approximation is reached.

The convergence on the successive meshes can be studied in more detail in Figure 9.3.17 on the facing page. Here the $L^{1}$-error and the associated EOC diagram for the horizontal velocity $v_{1}$, the vertical velocity $v_{2}$, and the temperature $T$ is displayed. As can be expected by the above-mentioned, the convergence of the velocity $v_{1}$ and the temperature $T$ is good, whereas no convergence for the velocity $v_{2}$ is observed. An explanation for this lacking convergence has been given above.
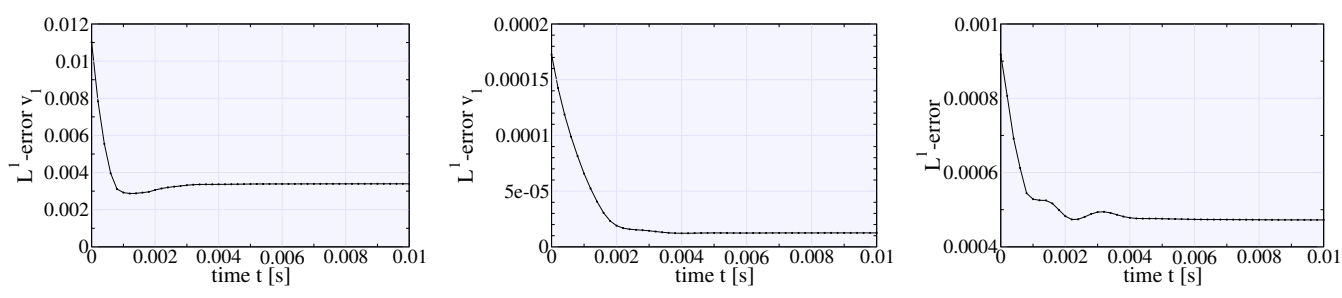

Figure 9.3.15: Blasius plate: temporal convergence of the time-dependent problem to a stationary state on the $50 \times 30 \times 1$ element mesh (shown is the $L^{1}$-error of the velocities $v_{1}$ (left) and $v_{2}$ (middle), and of the temperature $T$ (right)). 

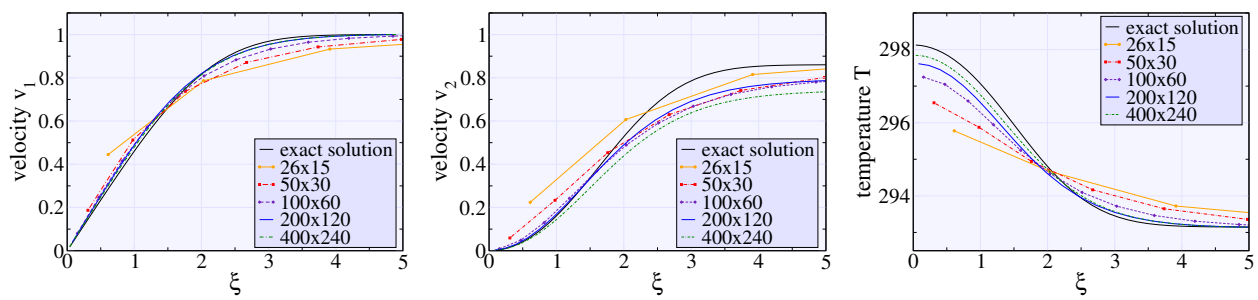

Figure 9.3.16: Blasius plate: profiles on the different meshes and exact curve of velocity $v_{1}$ (left), velocity $v_{2}$ (middle), and temperature $T$ (right).
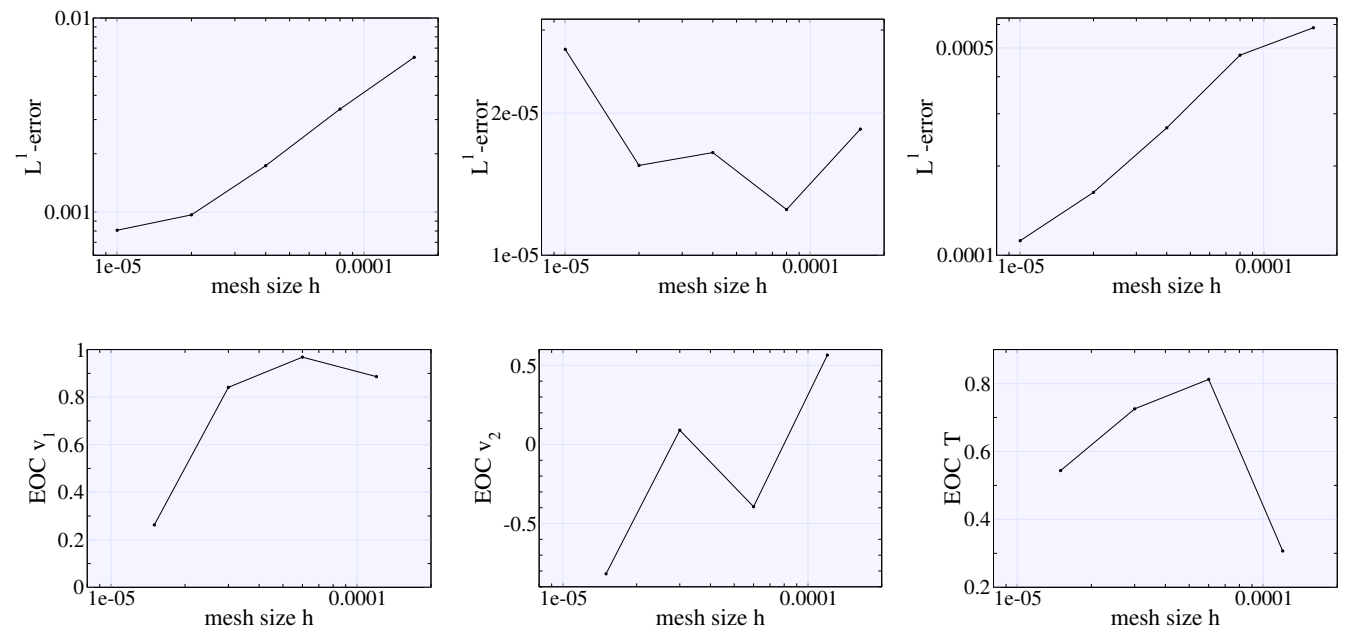

Figure 9.3.17: Blasius plate: $L^{1}$-error convergence (top) of the velocities $v_{1}$ (left), $v_{2}$ (middle), and the temperature $T$ (right) on the different meshes and the associated EOC values (below).

\subsubsection{Traveling Wave}

This test problem simulates a one-dimensional "shock wave" along a duct similar to the shock wave in the shock tube problem 9.2.2 on page 117, just that in viscid flows no shocks are possible. The transition between two states is always "smeared out" over a certain space. Strong gradients may be present but no actual shocks (see Figure 9.3.18 on the following page). As opposed to the other test problems for viscid flow, this traveling wave problem is not influenced by the interaction of fixed or moving walls with the fluid.

\section{Exact Solution}

An exact solution for this traveling wave problem has been established in [FR02]. The Navier-Stokes equations (3.3.4) in one dimension can be written in the form

$$
\frac{\partial}{\partial t} \mathbf{U}(x, t)+\frac{\partial}{\partial x} \mathbf{f}(\mathbf{U}(x, t))=\frac{\partial}{\partial x}\left(\mathbf{B}(\mathbf{U}(x, t)) \frac{\partial}{\partial x} \mathbf{U}(x, t)\right)
$$




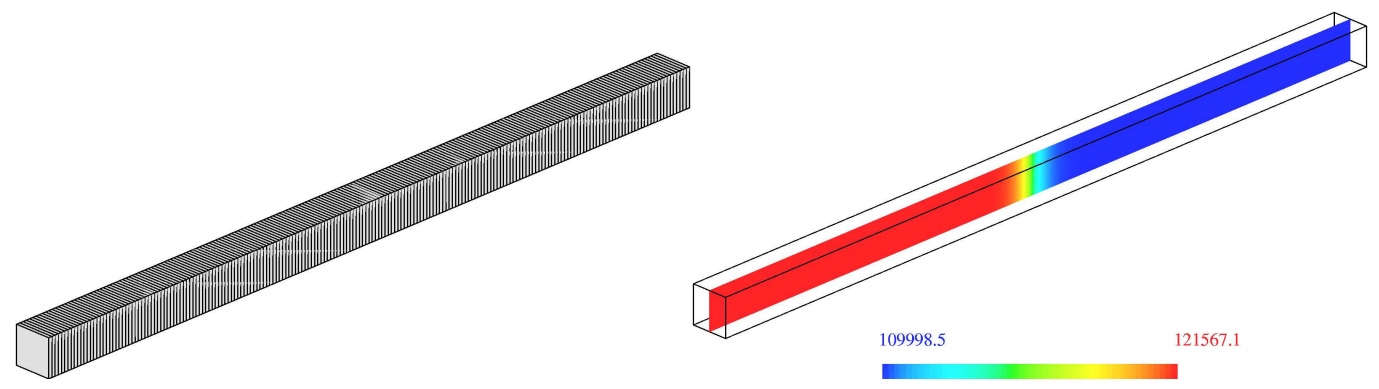

Figure 9.3.18: Traveling wave: the $200 \times 1 \times 1$ element mesh (left) and a clipping plane of the computed pressure on the $1600 \times 1 \times 1$ element mesh (right).

with flux function $\mathbf{f}: \Psi \rightarrow \mathbb{R}^{3}$ and smooth degenerate viscosity matrix $\mathbf{B}: \Psi \rightarrow \mathbb{R}^{3 \times 3}$ derived from (3.3.4), with the set of states $\Psi$ defined in (3.3.2). We are interested in solutions of the form

$$
\mathbf{U}(x, t)=\phi(x-s t)
$$

with $s$ constant, and

$$
\phi(-\infty)=\mathbf{U}^{-}, \quad \phi(\infty)=\mathbf{U}^{+}
$$

with left and right state $\mathbf{U}^{-}$and $\mathbf{U}^{+}$, that satisfy the Rankine-Hugoniot jump condition (see e.g. [Krö97] for this condition). Under these assumptions on $\mathbf{U}(x, t)$ we obtain by integrating equation (9.3.4)

$$
\mathbf{B}(\phi) \dot{\phi}=\mathbf{f}(\phi)-s \phi-q
$$

with the integration constant $q$ being equal to

$$
q=\mathbf{f}\left(\mathbf{U}^{-}\right)-s \mathbf{U}^{-}=\mathbf{f}\left(\mathbf{U}^{+}\right)-s \mathbf{U}^{+}
$$

and the boundary conditions given by (9.3.5). This ODE can now be solved following [FR02].

\section{Setup of the Numerical Experiment}

The viscosity $\eta$ and thermal conductivity $\lambda$, and also the data for the specific heat capacity at constant pressure $c_{p}$, are prescribed according to the values for air at a temperature of $T:=500 \mathrm{~K}$ :

$$
\begin{aligned}
\eta & :=3.662 \cdot 10^{-5} \frac{\mathrm{N} \mathrm{s}}{\mathrm{m}^{2}} \\
\lambda & :=5.564 \cdot 10^{-2} \frac{\mathrm{N}}{\mathrm{s} \mathrm{K}} \\
c_{p} & :=1093 \frac{\mathrm{m}^{2}}{\mathrm{~s}^{2} \mathrm{~K}}
\end{aligned}
$$

But apart from these settings, no temperature dependency of these material constants has been assumed. The adiabatic exponent $\gamma$ is as usual:

$$
\gamma:=1.4
$$


The computational domain is (see Figure 9.3.19)

$$
\Omega:=[-0.0002 ; 0.005] \times[0 ; 0.00002] \times[0 ; 0.00002] .
$$

The boundary values at the left and right-hand side of the domain are set according to the left and right state of the traveling wave:

$$
\begin{aligned}
\rho_{\text {left }} & :=0.7518 \frac{\mathrm{kg}}{\mathrm{m}^{3}} \\
v_{1, \text { left }} & :=33.747 \frac{\mathrm{m}}{\mathrm{s}} \\
p_{\text {left }} & :=121568.6 \mathrm{~Pa} \\
\rho_{\text {right }} & :=0.7 \frac{\mathrm{kg}}{\mathrm{m}^{3}} \\
v_{1, \text { right }} & :=0 \frac{\mathrm{m}}{\mathrm{s}} \\
p_{\text {right }} & :=110000 \mathrm{~Pa}
\end{aligned}
$$

All other boundaries are treated as slip walls and therefore the calculation can be regarded as quasi one-dimensional.

The initial values are given by the exact solution for the time $t=0$.

In order to save computational time, only the part of the mesh around the traveling wave itself is computed, the constant left and right states are not accounted for. This results in a moving computational domain of $x=0.0004$ length for the coarsest mesh down to $x=0.0001$ length for the finest mesh, with associated mesh sizes of $200 \times 1 \times 1$ to $3200 \times 1 \times 1$. Thus, with this technique a mesh width of $\Delta x=2 \cdot 10^{-6}$ down to $\Delta x=3.125 \cdot 10^{-8}$ could be achieved. The exact solution of the wave itself has an extension of about $2 \cdot 10^{-5}$. It follows that there are 10 elements up to 640 elements within the wave profile.

The final time is set to $t_{\text {end }}:=0.00001 \mathrm{~s}$, this corresponds to a propagation of the wave by $l \approx 0.0049 \mathrm{~m}$.

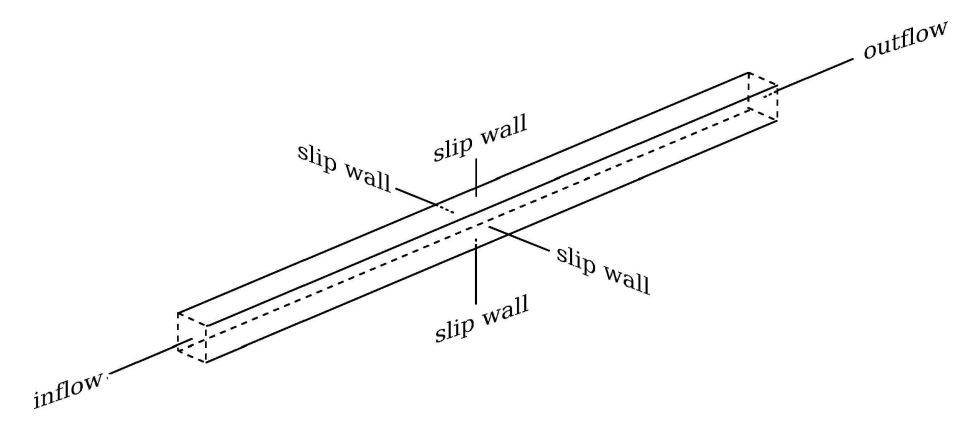

Figure 9.3.19: Traveling wave: boundary conditions and domain $\Omega$ for the experimental setup.

\section{Results}

The exact solution makes it clear that the regular counterpart of the shock wave from the lower state $\mathbf{U}^{-}$to the upper state $\mathbf{U}^{+}$is a smooth transition (see Fig- 
ure 9.3.20). This smooth curve is approximated very well by the numerical solutions on the finest meshes. The $L^{1}$-error and EOC plots in the next Figure 9.3.21 confirm this. The reduction in the EOC on the two finest meshes are due to round-off errors from the moving mesh.

As can be seen by the mesh size in $x$-direction $\left(\Delta x=2 \cdot 10^{-6}\right.$ down to $\Delta x=$ $\left.3.125 \cdot 10^{-8}\right)$, the sizes of the finer meshes can not be reached by any of our meshes for the two-stroke engine simulation. Therefore, a detailed resolution of a shock wave in the viscid flow regime is not possible in our calculations. There are just not enough elements in the zone of the traveling wave itself.

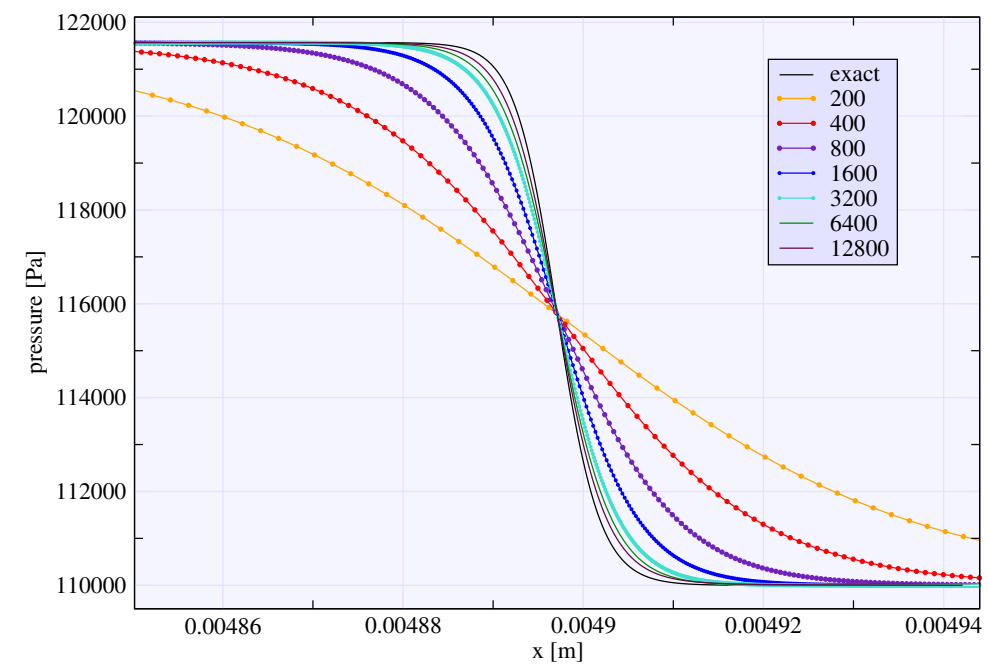

Figure 9.3.20: Traveling wave: pressure profile on the different meshes and exact pressure at $t=.00001$.
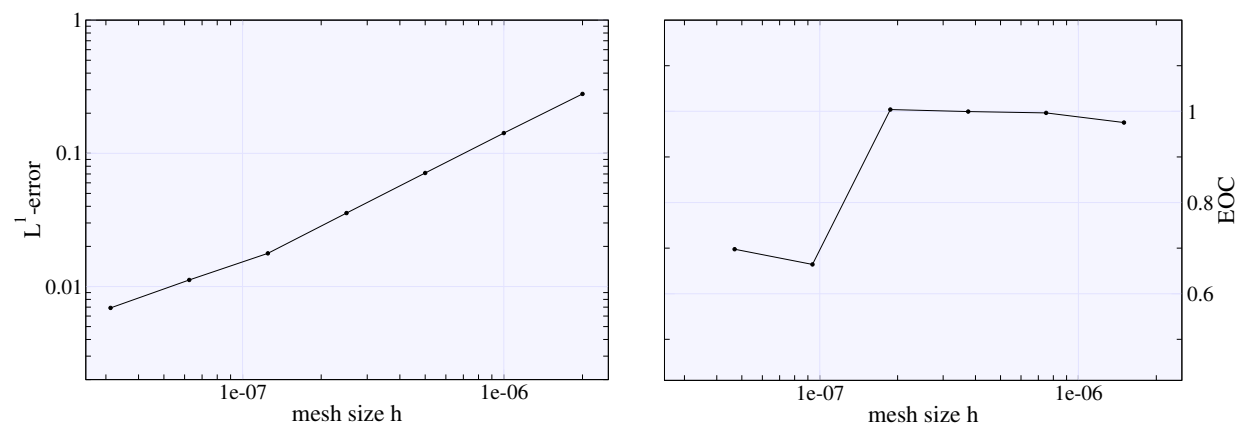

Figure 9.3.21: Traveling wave: $L^{1}$-error of the pressure (left) and the associated EOC (right). 


\subsection{Mesh Issues}

Now that we have validated the numerical scheme regarding the convective and viscous fluxes, we need to check its correct behavior with respect to the proposed mesh treatment. Namely, the grid merging method (see Section 5.1 on page 65 for an in depth description of this method) and the dynamic mesh adaption technique (as presented in Section 6.2 on page 91) are studied within the next three test problems.

\subsubsection{Grid Merging: A Sudden Expansion}

The grid merging with the patched grid method is, by construction, mass conservative (compare Section 5.1 on page 65 ). But is has to be verified that other flow quantities, as e.g. massflow, are treated correctly. In order to study this, a three-dimensional flow through a duct with a sudden expansion is observed with three different mesh setups (see Figure 9.4.1). Also, the virtual splitting approach (see Paragraph 5.1.5 on page 68) for the treatment of the elements with boundary area $A_{\text {bnd }}$ (see equation (5.1.1)) is compared to the standard method without virtual splitting.

A viscous flow is considered, and so the Navier-Stokes equations (3.3.4) are the appropriate mathematical model for this problem.

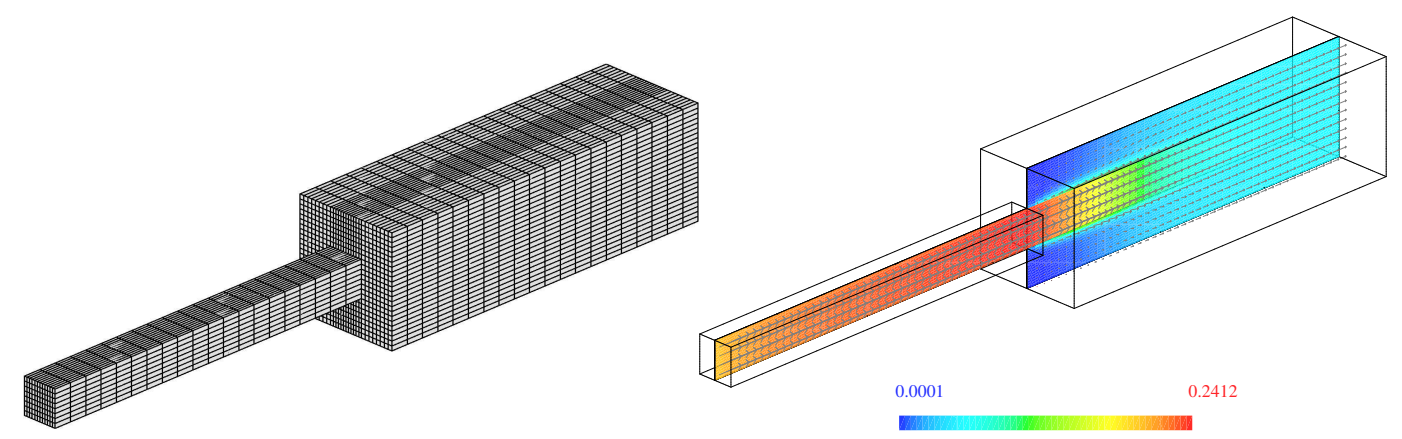

Figure 9.4.1: Sudden expansion: the $40 \times 8 / 24 \times 12 / 28$ element mesh (left) and a clipping plane of the computed Mach number and velocity vectors at time $t_{\text {end }}=0.0006 \mathrm{~s}$ on the $80 \times 16 / 48 \times 24 / 56$ element mesh (right).

\section{Validation of the Numerical Solution}

The numerical solutions obtained with the help of the grid merging algorithm is compared to the solution on a mesh without the need of this technique, but on an identical geometry setup.

\section{Setup of the Numerical Experiment}

We study a viscous flow where the viscosity $\eta$, the thermal conductivity $\lambda$, and the specific heat capacity at constant pressure $c_{p}$ are set to the values of air at standard 
temperature and pressure and no further temperature dependency is assumed:

$$
\begin{aligned}
\eta & :=1.834 \cdot 10^{-5} \frac{\mathrm{Ns}}{\mathrm{m}^{2}} \\
\lambda & :=2.540 \cdot 10^{-2} \frac{\mathrm{N}}{\mathrm{s} \mathrm{K}} \\
c_{p} & :=1004.8 \frac{\mathrm{m}^{2}}{\mathrm{~s}^{2} \mathrm{~K}}
\end{aligned}
$$

The adiabatic exponent $\gamma$ is set as in the above-stated test cases:

$$
\gamma:=1.4
$$

The computational domain

$$
\Omega:=[-0.1 ; 0] \times[0.01 ; 0.02] \times[0.01 ; 0.02] \cup[0 ; 0.1] \times[0 ; 0.03] \times[0 ; 0.03]
$$

together with the boundary types can be seen in Figure 9.4.2 on the next page.

The initial state of the flow is given by a compression profile similar to the one used in the first inviscid test case 9.2.1 on page 114. The profile is sinusoidal, has its peak right at the inflow boundary and the ambient pressure and density is reached at the transition from small to big duct (cf. Figure 9.4.3 on the next page). The detailed profile of the compression wave is described by (cf. 9.2.1 on page 114):

$$
\begin{array}{rlr}
\rho_{0} & :=1.2039 \frac{\mathrm{kg}}{\mathrm{m}^{3}} \\
p_{0} & :=101325 \mathrm{~Pa} & \\
p(x) & := \begin{cases}\left(p_{\max }-p_{0}\right) \cos \left(\frac{\pi}{2} 10(x+0.1)\right)+p_{0} & \text { for } x \leq 0 \\
p_{0} & \text { otherwise }\end{cases} \\
p_{\max } & :=1.2 p_{0} & \\
v_{1}(x) & =\frac{2}{\gamma-1} c_{0}\left(\left(\frac{p(x)}{p_{0}}\right)^{\frac{\gamma-1}{2 \gamma}}-1\right) & \\
\rho(x) & =\rho_{0}\left(\frac{p(x)}{p_{0}}\right)^{\frac{1}{\gamma}}
\end{array}
$$

with the speed of sound in the ambient fluid $c_{0}:=\sqrt{\frac{\gamma p_{0}}{\rho_{0}}}$.

The boundary values at the inflow boundary are given by the corresponding values at the peak of the compression profile:

$$
\begin{aligned}
\rho_{\text {in }} & :=\rho_{\max } \\
v_{1, \text { in }} & :=v_{1, \max }
\end{aligned}
$$

and the outflow values by the ambient ones:

$$
p_{\text {out }}:=p_{0}
$$

The additional boundary data is determined using the boundary treatment described in Section 4.7 on page 55 . 
The final time $t_{\text {end }}$ is chosen such that no influence from the boundaries can reach the contact interface.

Three different types of meshes are in use (see Figure 9.4.4 on the following page). The first kind does not need any grid merging at the interface from small to large duct. No hanging knots are present (Figure 9.4.4 (left)). The second type has hanging knots, therefore, the interface small to large duct is connected via grid merging, but there is no boundary area $A_{\text {bnd }}$ (see equation (5.1.1)) in any of the connecting faces (Figure 9.4.4 (middle)). In the last type of grid we even have $A_{\text {bnd }} \neq 0$ (Figure 9.4 .4 (right)). All three types of meshes have a resolution of $10 \times 2 / 6 \times 3 / 7$ (with 240 elements) up to $160 \times 32 / 96 \times 48 / 112$ (with 1 million elements).

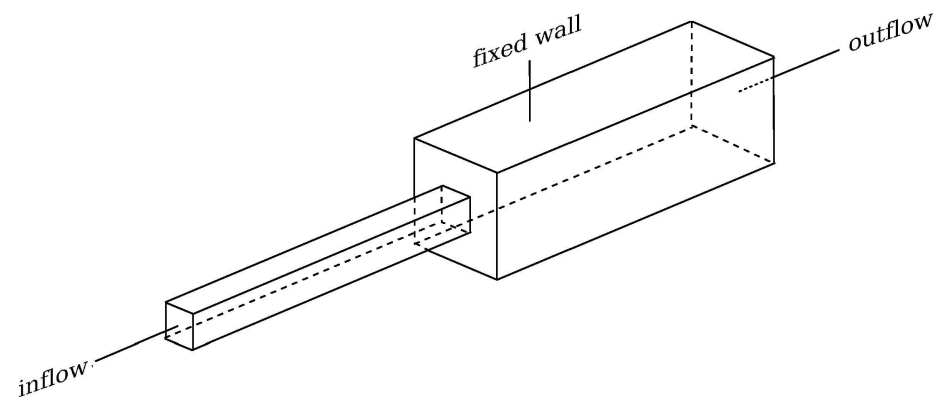

Figure 9.4.2: Sudden expansion: boundary conditions and domain $\Omega$ for the experimental setup.

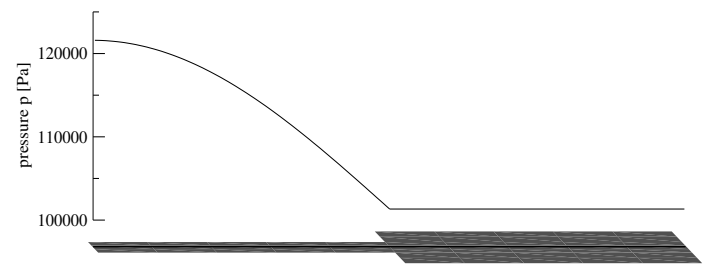

Figure 9.4.3: Sudden expansion: initial pressure distribution $p_{0}(x)$ along the center of the domain $(y=z=.015)$.

\section{Results}

As test item the total massflow $m$ through the connection face from small to large duct is taken. This is based on the fact that this parameter is crucial in our two-stroke engine simulation. The massflow through the engine affects all important characteristics, as e.g. the scavenge ratio $S R$ and trapping efficiency $T E$ (compare Paragraph 2.3.2 on page 13). In Figure 9.4.5 (left) the convergence of the total massflow $m_{h}^{\text {type }}$ on the different types of meshes can be seen. The black curve 

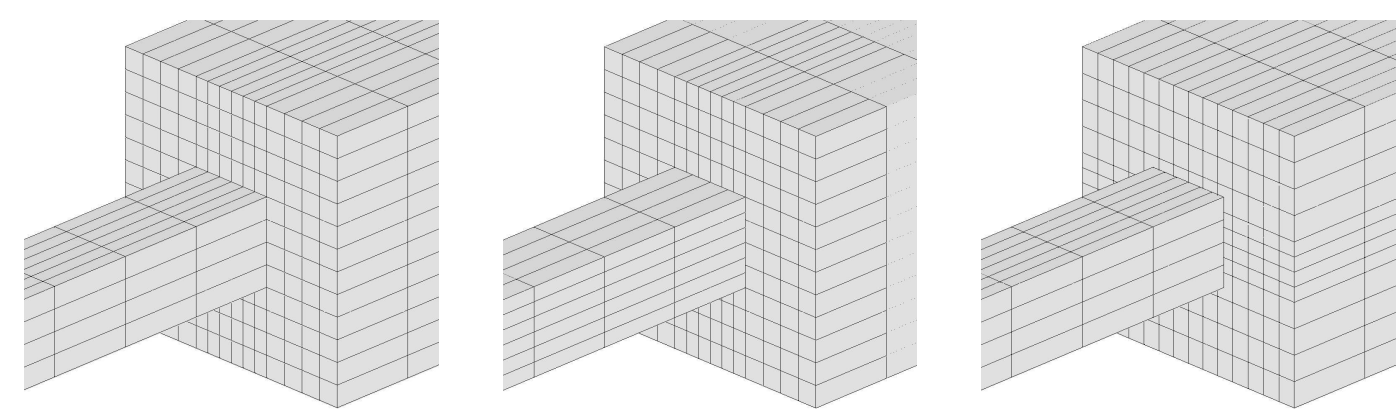

Figure 9.4.4: Sudden expansion: the three different types of meshes, type 1 with an exact connection (left), type 2 with exact boundary connection (middle), and type 3 with arbitrary connection (right) (the $20 \times 4 / 12 \times 6 / 14$ element mesh is shown each time).

shows the convergence history of $m_{h}^{\text {type } 1}$ of the meshes with exact connection. The exact value of the total massflow is approximated and seems to be located around $m_{0} \approx 0.005 \mathrm{~g}$. The yellow curve represents the total massflow $m_{h}^{\text {type } 2}$ on the meshes with hanging knots but exact boundary. This data is almost identical to the one on the meshes with exact connection. This can be explained by the fact that the flow in the small tube is almost one-dimensional (apart from the small boundary layer on the walls). Therefore, the values in the elements in the small tube right at the connecting face is virtually identical. Thus, an interpolation between these values, as is done by the grid merging method, does not alter the solution much. The red curve displays the values $m_{h}^{\text {type } 3 \text {, no vel.split. }}$ on the meshes with arbitrary connection and no virtual splitting (for the virtual splitting method cf. Paragraph 5.1.5 on page 68). A convergence is clearly visible, but the absolute error to the exact massflow $m_{0}$ is always larger than on the mesh without hanging knots:

$$
\left|m_{h}^{\text {type } 3, \text { no vel.split. }}-m_{0}\right|>\left|m_{h}^{\text {type } 1}-m_{0}\right|
$$

This is different for the method with virtual splitting. Not only that the error to the solution on the meshes without hanging knots is smaller (see Figure 9.4.5 (right)):

$$
\left|m_{h}^{\text {type } 3 \text {, vel.split. }}-m_{h}^{\text {type } 1}\right|<\left|m_{h}^{\text {type } 3 \text {, no vel.split. }}-m_{h}^{\text {type } 1}\right|
$$

but also the absolute error is smaller:

$$
\left|m_{h}^{\text {type 3, vel.split. }}-m_{0}\right|<\left|m_{h}^{\text {type } 1}-m_{0}\right|
$$

Thus, the grid merging method with virtual splitting is superior to the grid merging method without virtual splitting in the general case.

\subsubsection{Dynamic Mesh Adaption: The Forward Facing Step}

A test case that has been simulated by a multitude of codes is the forward facing step (see Figure 9.4.6 on the next page). A supersonic flow through a channel 

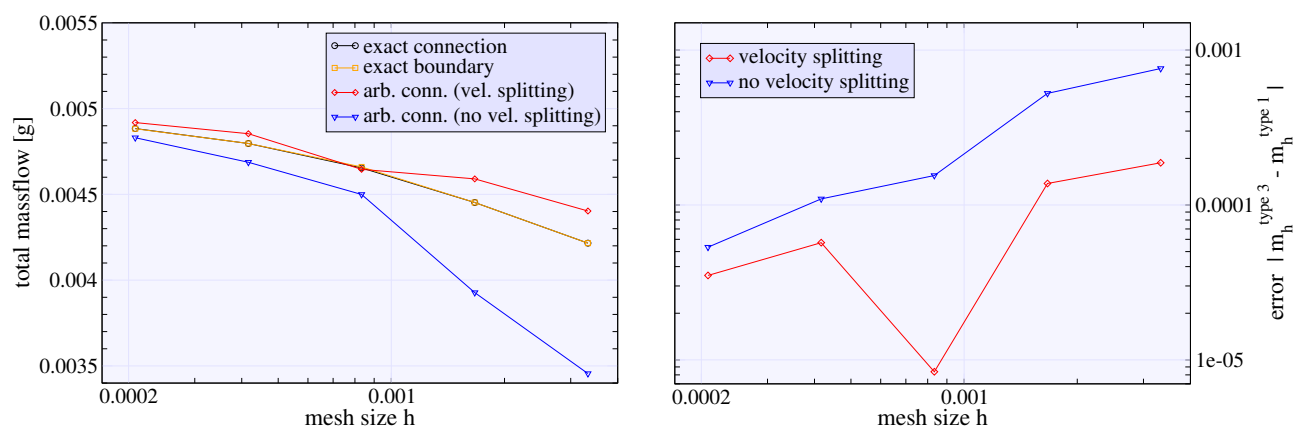

Figure 9.4.5: Sudden expansion: convergence of the total massflow on the different types of meshes (left) and error for the two different approaches on the type 3 meshes (right) for decreasing mesh size $h$.

encounters a blunt object on its way. The problem is characterized by the presence of strong shock waves, namely a bow shock in front of the step, and its successive reflections on the walls. It is an ideal test problem for the study of a local mesh refinement. A few areas with steep gradients need a high resolution, whereas wide regions can be approximated by a relatively coarse mesh, thus saving computational time without losing accuracy. As in Section 9.2 on page 114 an inviscid fluid is considered and the Euler equations (see Paragraph 3.3.1 on page 31) provide the appropriate mathematical model.
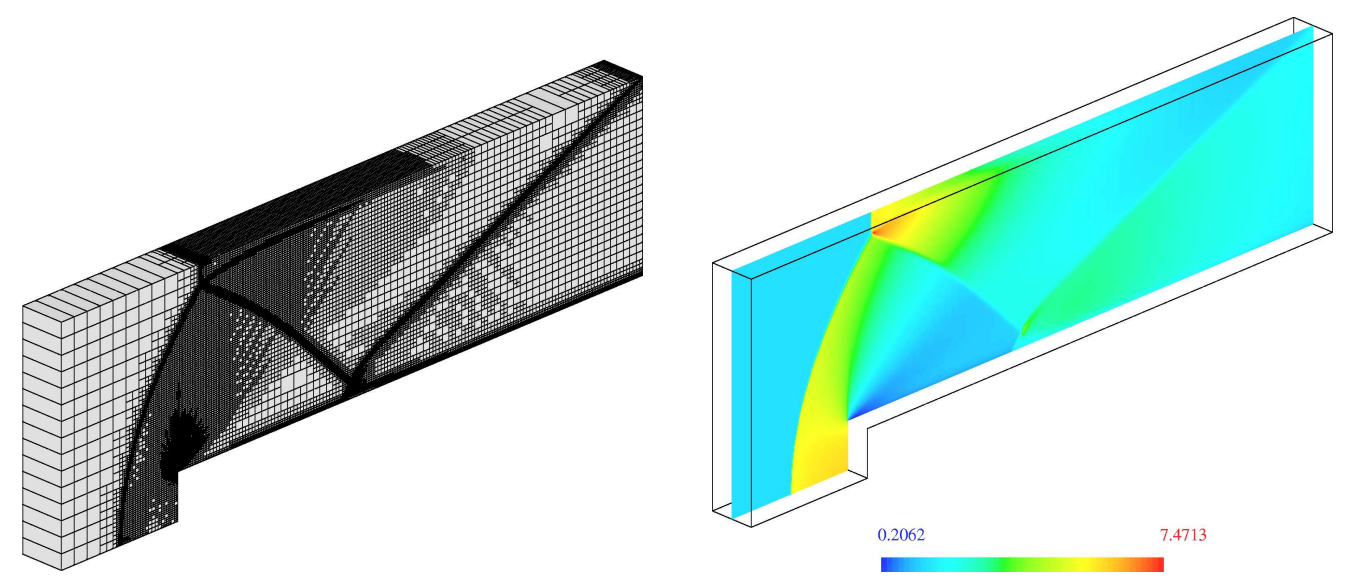

Figure 9.4.6: Forward facing step: the $45 \times 15 / 12 \times 1$ element mesh with refinement up to level $l_{\text {ref }}=4$ (left) and a clipping plane of the computed density on the same mesh (right) at time $t=2.5$.

\section{Validation of the Numerical Solution}

As no exact solution of this problem is known, a validation of our results is possible by comparison with results from other numerical schemes. For example in 
[Sch99] and [Be00] this test case has been studied and detailed results are given for different times $t$. Also, a validation of the dynamic mesh adaption is feasible by comparing its results with the numerical solution obtained with a global refinement of the mesh.

\section{Setup of the Numerical Experiment}

A polytropic flow is simulated, the adiabatic exponent is set to the value for a diatomic gas:

$$
\gamma:=1.4
$$

The shape and boundary conditions of the computational domain

$$
\Omega:=[0 ; 3] \times[0.2 ; 1] \times[0 ; 0.2] \cup[0 ; 0.6] \times[0 ; 0.2] \times[0 ; 0.2]
$$

are sketched in Figure 9.4.7 on the facing page.

As in the reference computation, all variables are given in dimensionless values. So, the boundary values for the inflow and outflow boundary are fixed as follows:

$$
\begin{array}{ll}
\rho_{\text {in }}:=\rho_{\text {out }} & :=1.4 \\
p_{\text {in }}:=p_{\text {out }} & :=1 \\
v_{1, \text { in }} & :=v_{1, \text { out }}:=3
\end{array}
$$

It follows for the speed of sound $c=\sqrt{\frac{\gamma p}{\rho}}=1$, and thus a Mach 3 flow is simulated. In order to simulate a quasi two-dimensional flow, the boundary conditions for the front and rear boundary $P_{\text {front }}=\{z=0\}$ and $P_{\text {rear }}=\{z=0.2\}$ are slip wall conditions.

The initial conditions are identical to the boundary conditions at the inflow and outflow boundaries.

As macro meshes a $45 \times 15 / 12 \times 1$ element mesh and a $180 \times 60 / 48 \times 1$ element mesh is used. The maximum refinement level is $l_{\text {ref }}:=5$. In the case of the $45 \times 15 / 12 \times 1$ element mesh this corresponds to a mesh of 18 million elements and with the 9000 element macro mesh to a 300 million element mesh obtained by global refinement.

The parameter for the marking strategy (as described in Section 6.2.4 on page 94) are given as follows:

$$
\begin{array}{rll}
C_{\text {refine }} & :=0.9 \\
C_{\text {coarsen }} & :=0.4
\end{array}
$$

The mesh is checked for refinement every $4^{\text {th }}$ time step and for coarsening every $32^{\text {nd }}$ time step.

\section{Results}

In Figures 9.4 .8 on page 153 and 9.4 .9 on page 154 it can be seen very nicely how the dynamic mesh adaption (on the upper part of the figures) follows the structure of the solution (displayed is a clipping plane of the density) in the course of the calculation. Also, the comparison of the solution on the adaptive mesh, refined up 


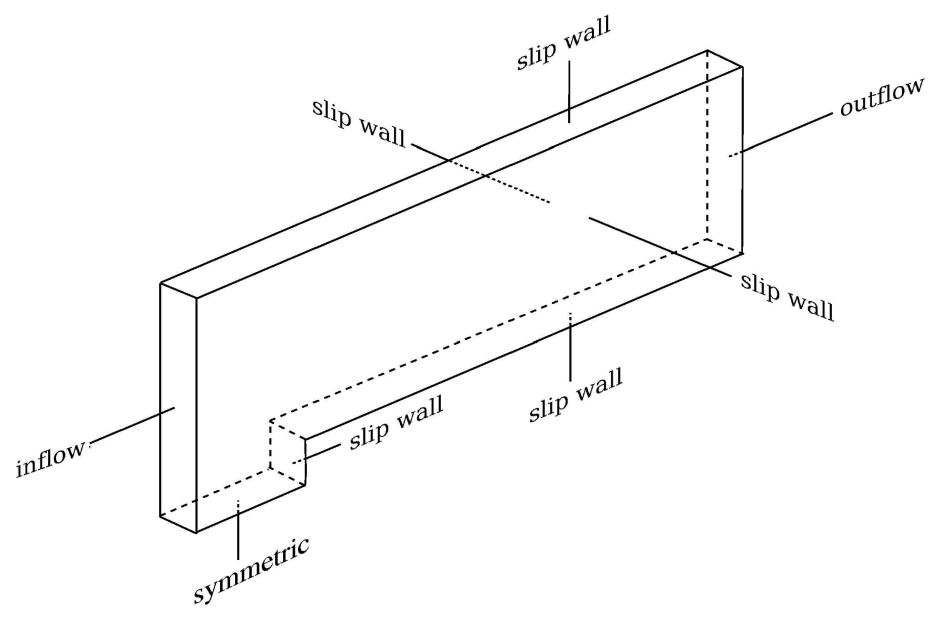

Figure 9.4.7: Forward facing step: boundary conditions and domain $\Omega$ for the experimental setup.

to refinement level $l_{\text {ref }}=4$ (middle part of the figures) to the one on the, also four times, globally refined mesh (bottom part) shows how the important flow features with steep gradients are captured by the adaptive mesh. The shock wave and its position are resolved exactly. Where the solution shows gentle gradients, e.g. behind the bow shock after the step, the accuracy is reduced to save computational time.

How the choice of the refinement parameters for the marking strategy controls the number of elements is demonstrated in Figure 9.4.10 on page 155 for a refinement level of $l_{\text {ref }}=5$. The parameters range from $C_{\text {refine }}=1.1, C_{\text {coarsen }}=0.55$ with approximately 141000 elements (upper part of the figure), $C_{\text {refine }}=1.0, C_{\text {coarsen }}=$ 0.5 with 175000 elements (middle part), and $C_{\text {refine }}=0.9, C_{\text {coarsen }}=0.4$ with a little less than 590000 (bottom part). Especially, the steep rise of the number of elements in the last two cases is remarkable.

The effect of the level of adaption $l_{\text {ref }}$ is shown in Figures 9.4.11 on page 156 and 9.4.12 on page 157. The adaptive meshes (left-hand side of the figure) and the corresponding solution (right-hand side) are displayed for an adaption level from $l_{\text {ref }}=2$ up to $l_{\text {ref }}=5$ for the $45 \times 15 / 12 \times 1$ element mesh and for $l_{\text {ref }}=4$ and $l_{\text {ref }}=5$ for the $180 \times 60 / 48 \times 1$ element mesh. It can be seen, how the position and the structure of the shocks is enhanced with each further refinement level. In the two plots in Figure 9.4.13 on page 158 the efficiency of the dynamic adaptive mesh refinement can be estimated. On the left diagram the number of elements for a global mesh refinement versus the number of elements for the adaptive mesh refinement at time $t=3$ is given. On the corresponding diagram on the right-hand side of Figure 9.4.13 on page 158 this difference is even more impressive. At level $l_{\text {ref }}=1$, a little less than half of the elements is used with the adaptive refinement. But this figure is reduced down to 3.2 percent for refinement level $l_{\text {ref }}=5$. Thus, the computational time and the memory requirements would be about 30 times as much for the calculation on a globally refined mesh, assuming a linear dependency for the number of elements to time and memory needs.

Summarizing this, it could be shown that the dynamic adaptive mesh refinement 
is very efficient regarding computational time and memory demand, especially at higher refinement levels, and that the accuracy is only reduced in areas without important structures of the flow field.

\subsubsection{Boundary Fitting of the Dynamic Mesh Adaption: Channel with a Bump}

This test problem has been described in detail in Paragraph 9.2.4 on page 125. Here it shall be reviewed in the light of the dynamic mesh adaption. The concept of boundary fitting is presented in Paragraph 6.2 .5 on page 95. In this example its necessity becomes obvious.

\section{Validation of the Numerical Solution}

The solution with the boundary fitting algorithm and the one without it are compared to the numerical solution obtained on a globally refined mesh with the matching resolution.

\section{Setup of the Numerical Experiment}

The setup is described in Paragraph 9.2.4 on page 125. Here we only want to add the parameters concerning the dynamic mesh refinement. The coarsest mesh of $24 \times 10 \times 4$ elements is used as macro grid and is adaptively refined down to refinement level $l:=3$. Thus, it corresponds to the (globally) refined mesh of $192 \times 80 \times 4$ elements. The marking strategy of the mesh adaption (described in Section 6.2 .4 on page 94 ) is, as in the above studied problem of the forward facing step, controlled by:

$$
\begin{array}{rll}
C_{\text {refine }} & :=0.9 \\
C_{\text {coarsen }} & :=0.4
\end{array}
$$

Furthermore, the mesh is checked for refinement every $4^{\text {th }}$ time step and for coarsening every $32^{\text {nd }}$ time step.

\section{Results}

The numerical solution without the boundary fitting algorithm can be seen on the left-hand side of Figure 9.4.14 on page 158. If compared to the solution obtained on the globally fine mesh of Figure 9.4.14 (right) the presence of a second shock behind the sharp edge from the macro grid on top of the bump can be spotted. This additional shock is not present in the solution with the help of the boundary fitting algorithm (depicted in Figure 9.4.14 (middle)). This difference is even more obvious 

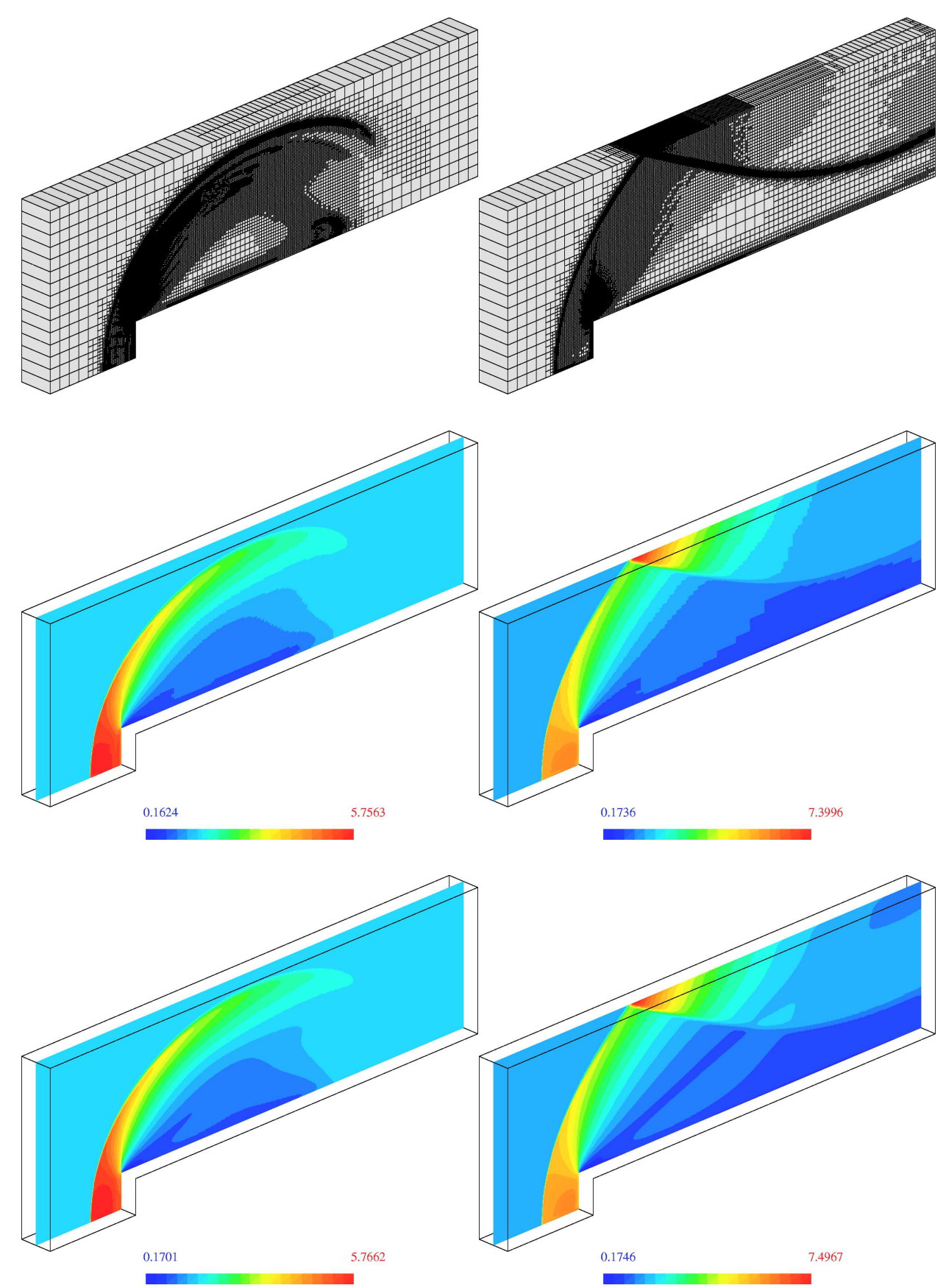

Figure 9.4.8: Forward facing step: the $45 \times 15 / 12 \times 1$ element mesh adaptively refined to level $l_{\text {ref }}=4$ (top), the solution obtained by adaptive refinement (middle), and the solution for global refinement (bottom) at time $t=0.5$ (left) and $t=1$ (right). 

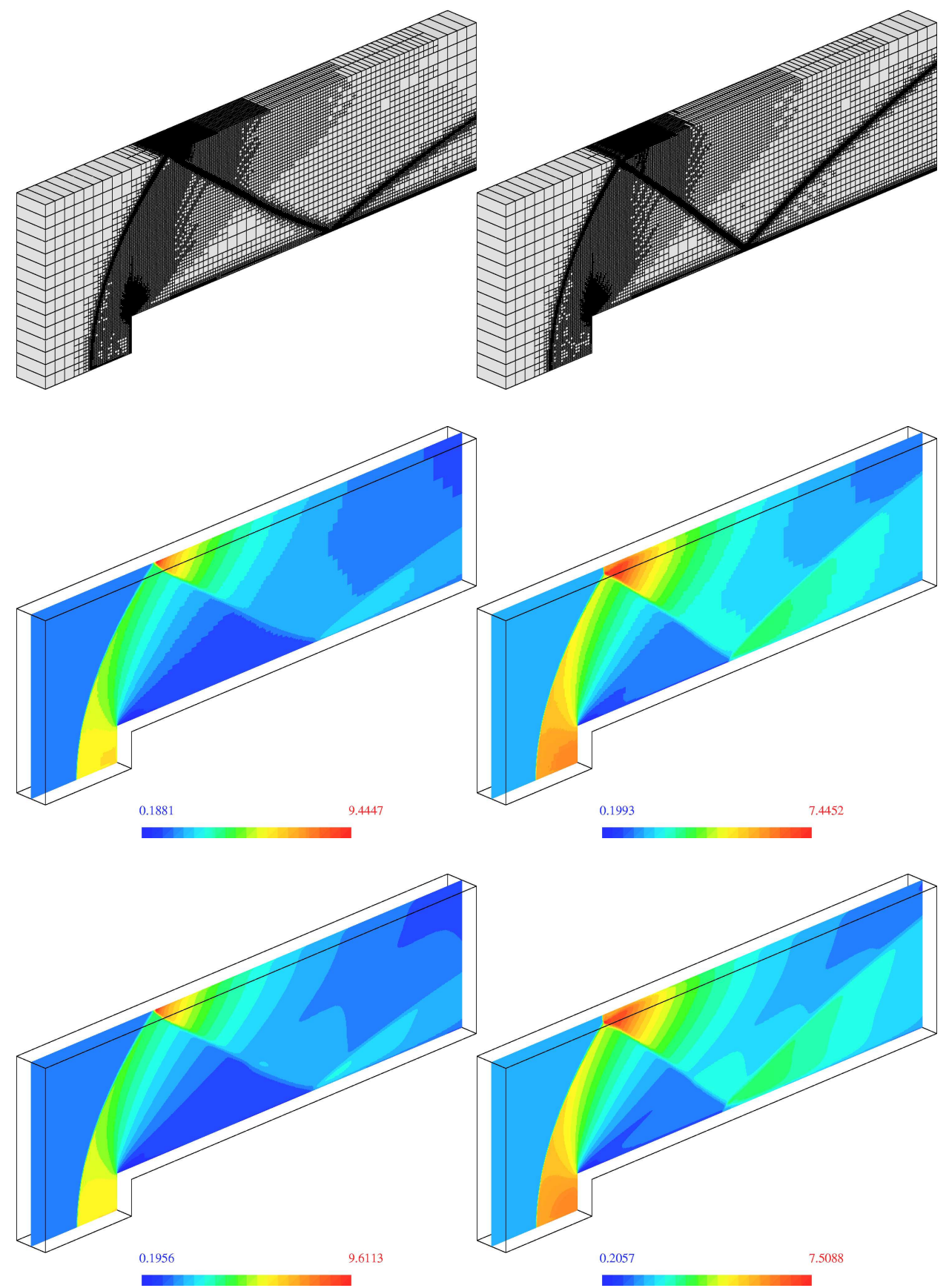

Figure 9.4.9: Forward facing step: the $45 \times 15 / 12 \times 1$ element mesh adaptively refined to level $l_{\text {ref }}=4$ (top), the solution obtained by adaptive refinement (middle), and the solution for global refinement (bottom) at time $t=1.5$ (left) and $t=2$ (right). 

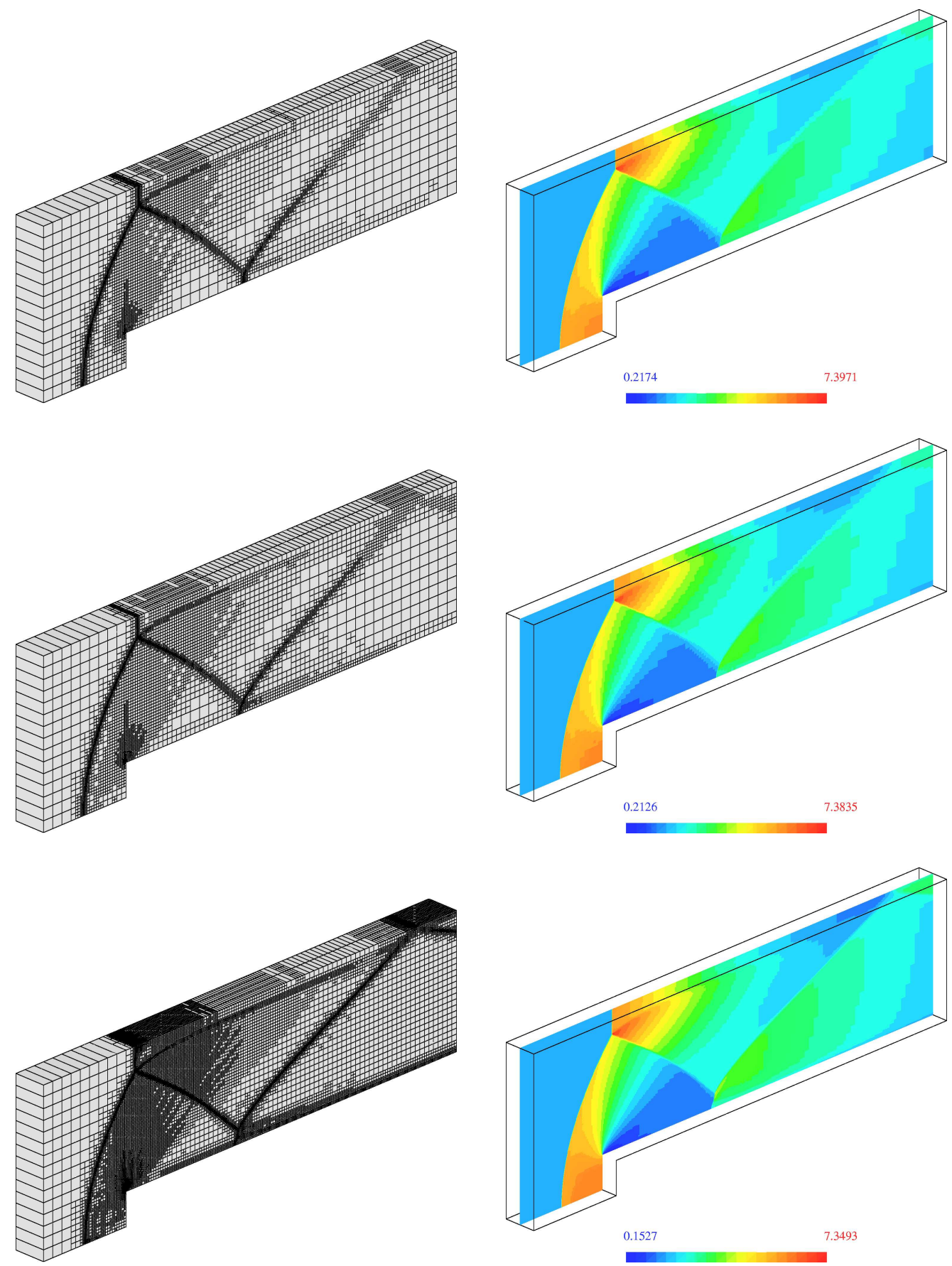

Figure 9.4.10: Forward facing step: the $45 \times 15 / 12 \times 1$ element mesh (left) and the calculated density (right) at time $t=3$ adaptively refined to level $l_{\text {ref }}=5$ with refinement constants $C_{\text {refine }}=1.1, C_{\text {coarsen }}=0.55$ (top), $C_{\text {refine }}=1.0, C_{\text {coarsen }}=0.5$ (middle), and $C_{\text {refine }}=0.9$, $C_{\text {coarsen }}=0.4$ (bottom). 

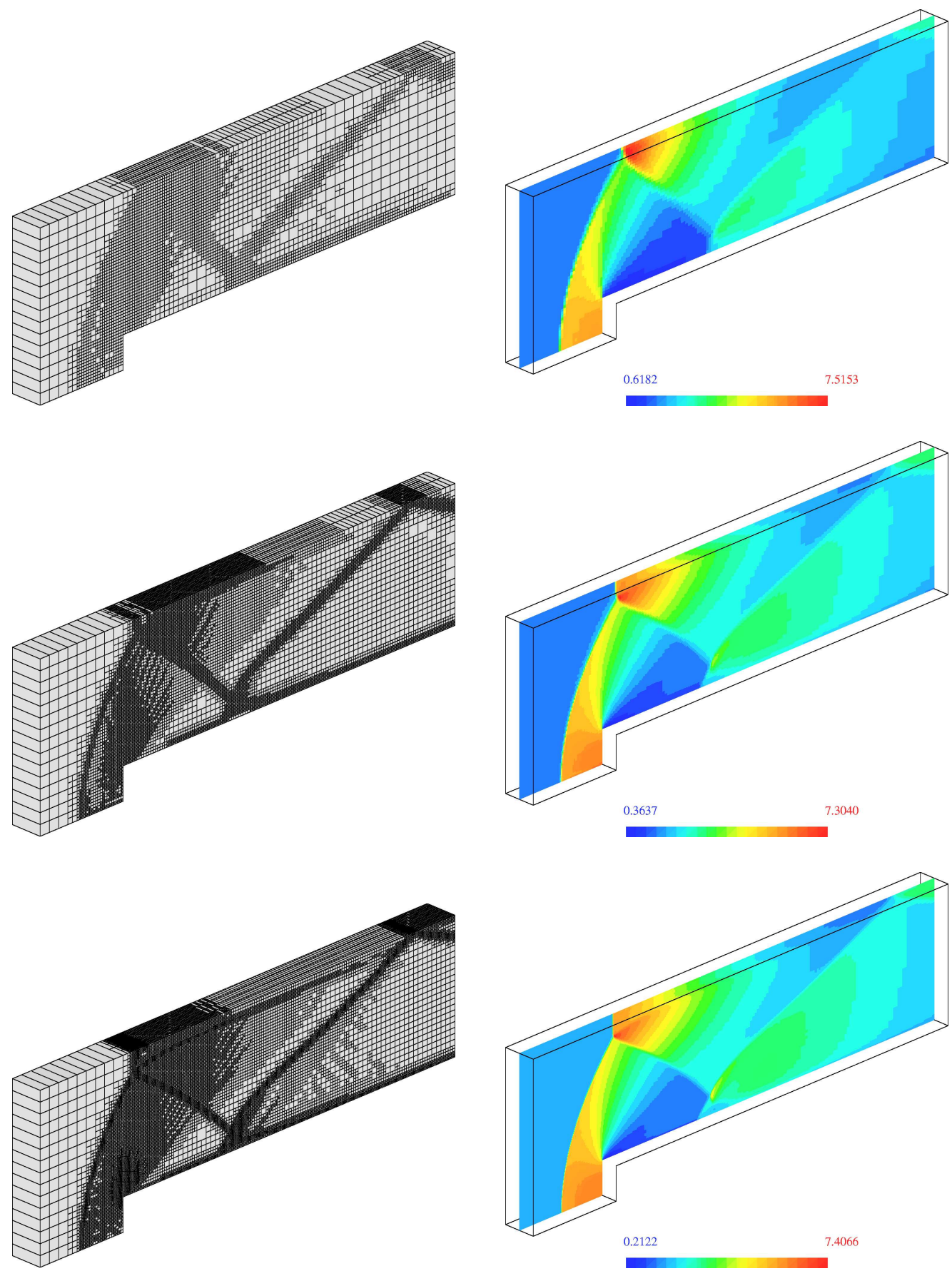

Figure 9.4.11: Forward facing step: the $45 \times 15 / 12 \times 1$ element mesh (left) and the calculated density (right) at time $t=3$ adaptively refined to level $l_{\text {ref }}=2$ (top), to level $l_{\text {ref }}=3$ (middle), and to level $l_{\text {ref }}=4$ (bottom). 

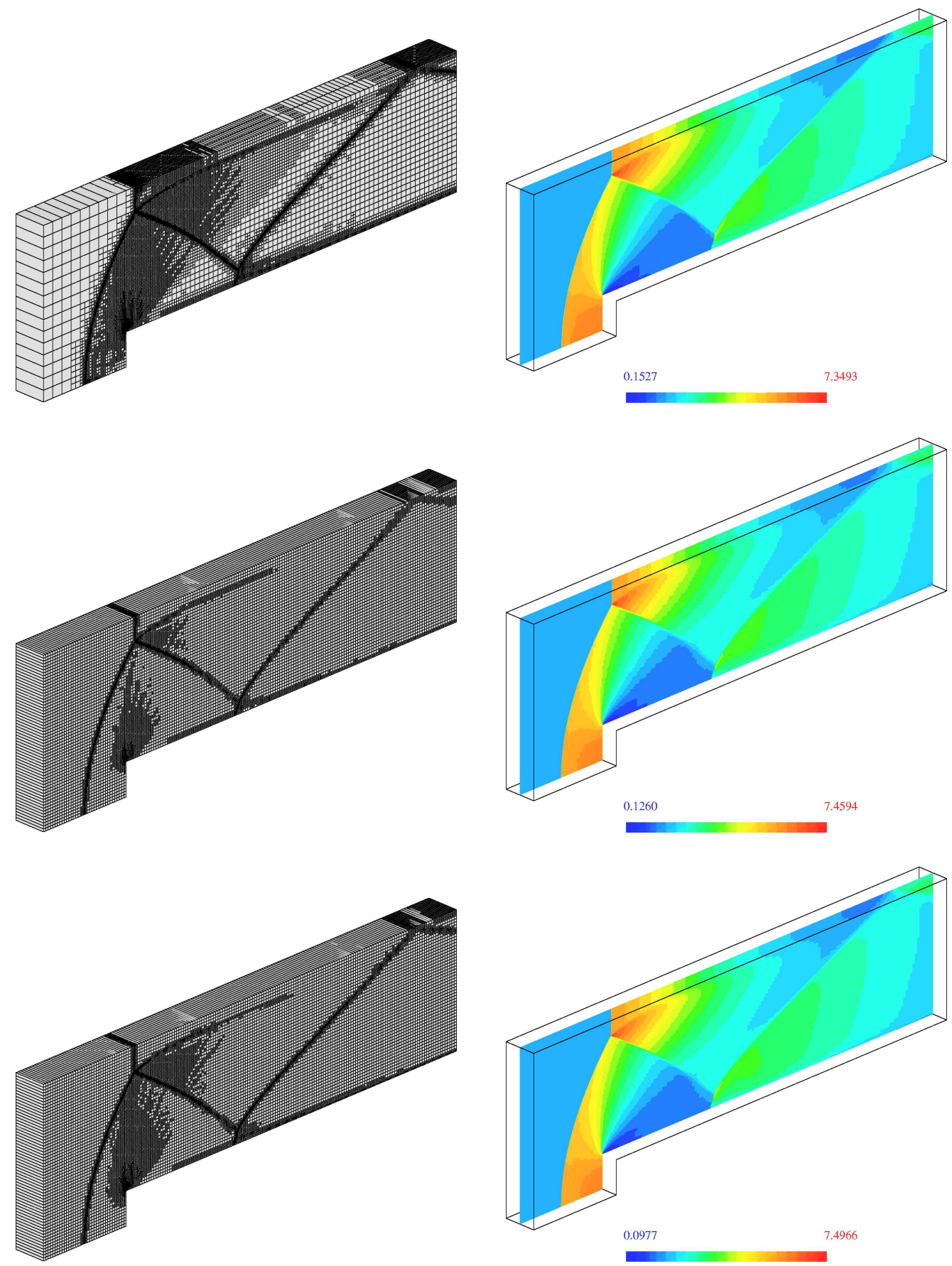

Figure 9.4.12: Forward facing step: the mesh (left) and the calculated density (right) at time $t=3$, for the $45 \times 15 / 12 \times 1$ element mesh adaptively refined to level $l_{\text {ref }}=5$ (top), and for the $180 \times 60 / 48 \times 1$ element mesh refined to to level $l_{\text {ref }}=4$ (middle), and to level $l_{\text {ref }}=5$ (bottom). 

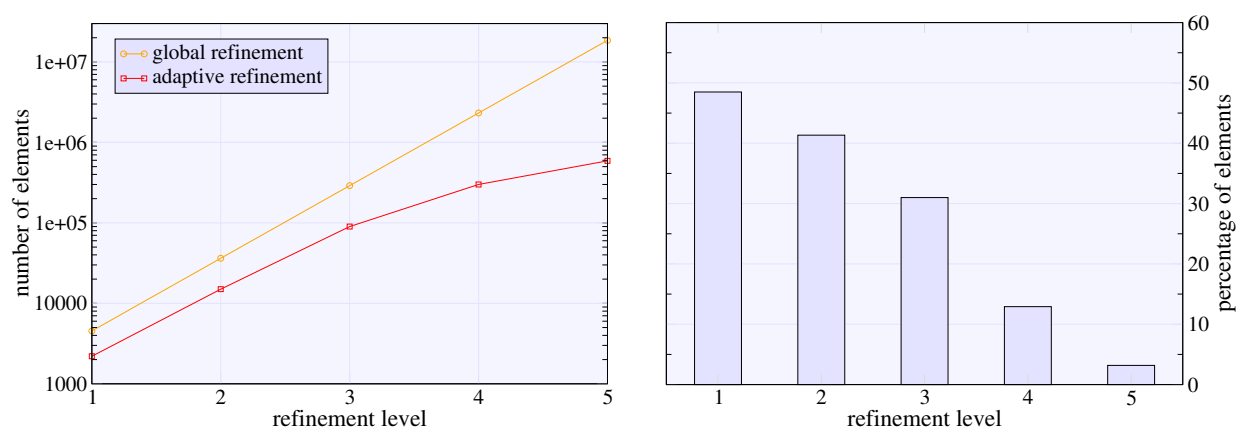

Figure 9.4.13: Forward facing step: number of elements at the different refinement levels for global and adaptive refinement (left) and the corresponding percentage of the number of elements for adaptive versus global refinement (right) at time $t=3$.

in the diagram in Figure 9.4.15 on the facing page. Displayed is the pressure along the bottom layer of elements and along the middle horizontal plane. The difference between the dynamic mesh adaption with boundary fitting and the globally fine mesh is hardly noticeable, whereas the pressure of the numerical solution without boundary fitting has a peak after each kink on the bump and decreases after the kink.

Summarizing, it can be said that the boundary fitting is definitely necessary in those cases where a flow along a round object is simulated. This is e.g. the case with the crank shaft or the cylinder in the two-stroke engine.

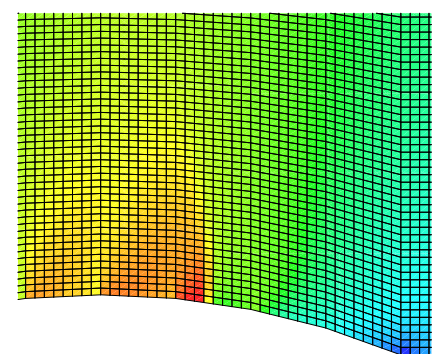

0.3453
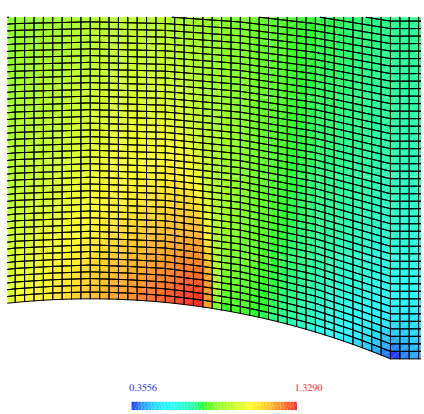

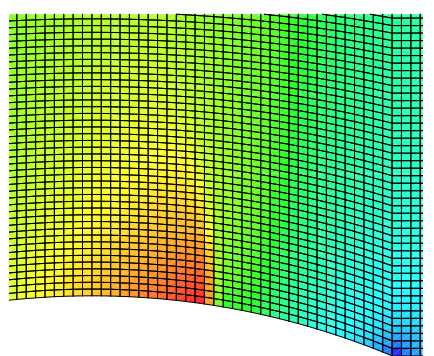

Figure 9.4.14: Channel with a bump: the Mach number contours without boundary fitting (left), with boundary fitting (middle), and on a globally fine mesh (right).

\subsection{In the Two-Stroke Engine Context}

\subsubsection{Straight Pipe attached to a Cylinder}

In [B196], a physical experiment can be found that is closely related to the flow out of, or into, a cylinder. A straight parallel pipe is attached to this cylinder 


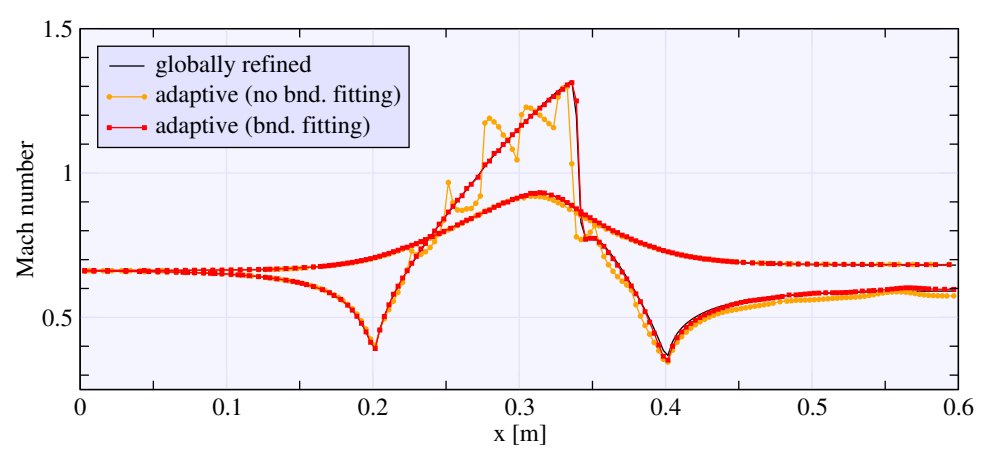

Figure 9.4.15: Boundary fitting: comparison of the Mach number plots for the solution without boundary fitting, with boundary fitting, and on a globally fine mesh.

(see Figure 9.5.1). The connection can be operated by a valve, that has opening and closing characteristics similar to a piston opening and closing of a port. The pressure wave, that is propagating along the pipe, is measured by two fast-response pressure transducers, which are positioned at the pipe. For our numerical experiment, we apply the Navier-Stokes equations (3.3.4) to simulate a viscous fluid.

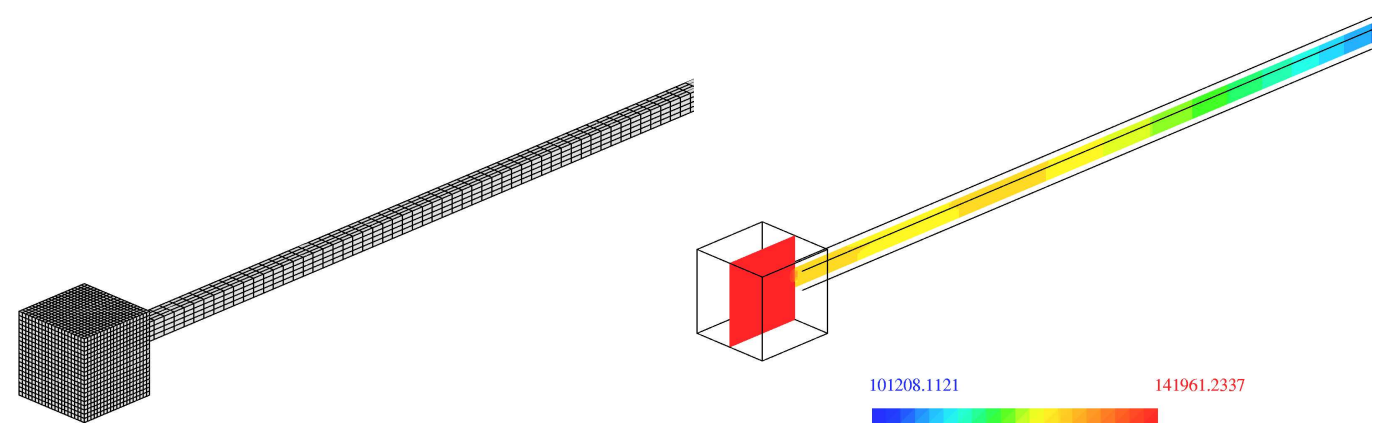

Figure 9.5.1: Exhaust pipe: the $205 \times 5 / 1 \times 5 / 1$ element mesh with refinement up to level $l_{\text {ref }}=2$ (left) and a clipping plane of the computed pressure on the four times refined mesh (right) at time $t=0.003$.

\section{Validation of the Numerical Solution}

In order to validate our numerical solution, we compare the numerical and experimental pressure profiles at the positions of the pressure transducers. Additionally, the total massflow can be measured and compared.

\section{Setup of the Exhaust Pipe Experiment}

The configuration of the experiment (taken from [B196]) is shown in Figure 9.5.2. The cylinder is box-shaped with a volume of $V_{\text {cyl }}:=912 \mathrm{~cm}^{3}$, the attached pipe has a diameter of $d_{\text {pipe }}:=25 \mathrm{~mm}$ length of $l_{\text {pipe }}:=5.901 \mathrm{~m}$ and opens to the atmosphere at 
the far end. Therefore, in the pipe we initially have ambient atmospheric conditions:

$$
\begin{aligned}
& T_{0, \text { pipe }}:=293.15 \mathrm{~K} \\
& p_{0, \text { pipe }}:=101325 \mathrm{~Pa}
\end{aligned}
$$

The positions of the two pressure transducers are given by $l_{\mathrm{p} 1}:=0.317$ and $l_{\mathrm{p} 2}:=$ 3.691. The valve operates in such a way that a port opening lasts for $0.008 \mathrm{~s}$, which is typical for a two-stroke engine at $3000 \mathrm{rpm}$. The temperature and the pressure of the cylinder is initially set to:

$$
\begin{aligned}
& T_{0, \text { cyl }}:=293 \mathrm{~K} \\
& p_{0, \text { cyl }}:=1.5 p_{\text {pipe }}=151987.5 \mathrm{~Pa}
\end{aligned}
$$

At time $t=0 \mathrm{~s}$ the valve starts to open, is fully open at time $t=0.004 \mathrm{~s}$, and closed again at $t=0.008 \mathrm{~s}$. The area $A$ of the port window is calculated by the following formula, that results from the intersection area of two identical circles with radius $r$ :

$$
\begin{aligned}
d & :=\left|2 r\left(1-\frac{t}{0.004}\right)\right| \\
\alpha & :=2 \cos \left(\frac{d}{2 r}\right) \\
A & =\alpha r^{2}-d r \sqrt{1-\left(\frac{d}{2 r}\right)^{2}}
\end{aligned}
$$

The data at the outflow boundary at the end of the pipe is set to:

$$
p_{\text {out }}:=101325 \mathrm{~Pa}
$$

The other values for the outflow boundaries are calculated according to the boundary treatment described in Section 4.7 on page 55 .

The mesh consists of $205 \times 5 / 1 \times 5 / 1$ elements and is successively refined up to level $l_{\text {ref }}=5$ (see Figure 9.5.1 on the page before for the mesh on refinement level $\left.l_{\text {ref }}=2\right)$.

Although no significant temperature differences are present, we use the temperature-dependent heat capacities $c_{p}$ and $c_{\mathrm{V}}$, adiabatic exponent $\gamma$, viscosity $\eta$, and thermal conductivity $\lambda$ of air (see Section 3.8 on page 40 ).

\section{Results of the Exhaust Pipe Experiment}

The numerical experiment is conducted with a mesh in 5 different refinement levels. Figure 9.5.3 on the next page shows the numerical compression wave in the pipe at the second pressure transducer. A convergence to a mesh-independent solution is clearly visible.

In Figure 9.5.4 on page 162 we can see the detailed pressure history in the cylinder (upper part of the figure) and at the first (middle part) and second (lower part) transducer. At the onset of the opening of the valve between cylinder and pipe 


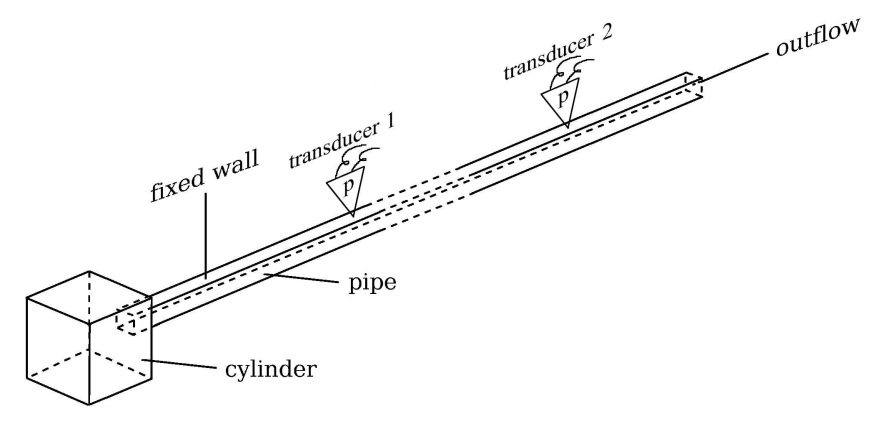

Figure 9.5.2: Exhaust pipe: boundary conditions and domain $\Omega$ for the experimental setup.

a compression wave is produced by the pressure gradient from cylinder and pipe (Figure 9.5.4 (top)). The pressure in the cylinder is decreasing at the same time. The ratio of final to initial cylinder mass is measured as 0.866 in the physical experiment and with 0.864 it is virtually identical in our simulation. However, the shape of the compression profile is not exactly reproduced, as can be seen in this upper part of the figure. At the first pressure transducer (middle part of Figure 9.5.4), one can see the compression wave on its way rightward and the reflected expansion wave from the far end of the pipe. This expansion wave is again reflected at the pipe-cylinder connection and superimposed at this first transducer. On the second measuring point (lower part of Figure 9.5.4), the compression wave arrives later, its reflection as expansion wave at the pipe end is seen earlier, and the reflection of this expansion wave at the closed valve passes again. A steep fronting, as explained in test case 9.2 .1 on page 114, is clearly visible in these graphs. Also, the timing of the passage of the waves is almost exact. Just the peak value of the expansion wave is slightly underestimated. This might be due to the outflow boundary condition. This boundary condition is replaced by the silencer in the engine simulation (see Paragraph 10.1.3 on page 169), thus eliminating this possible source of inaccuracy.

Thus, it can be seen that the physical experiment and the numerical simulation agree very well with each other.

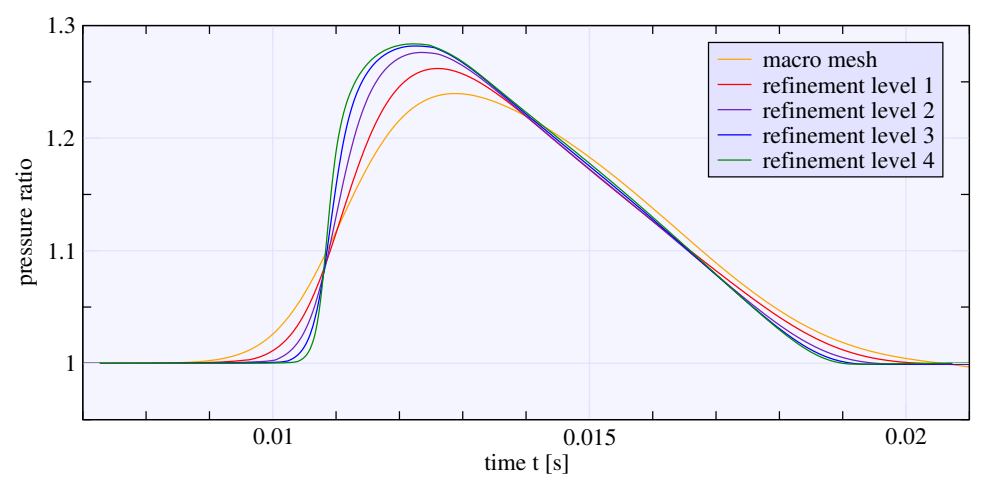

Figure 9.5.3: Exhaust pipe: convergence on the different meshes of the pressure profile at pressure transducer one for the compression pulse moving rightwards. 

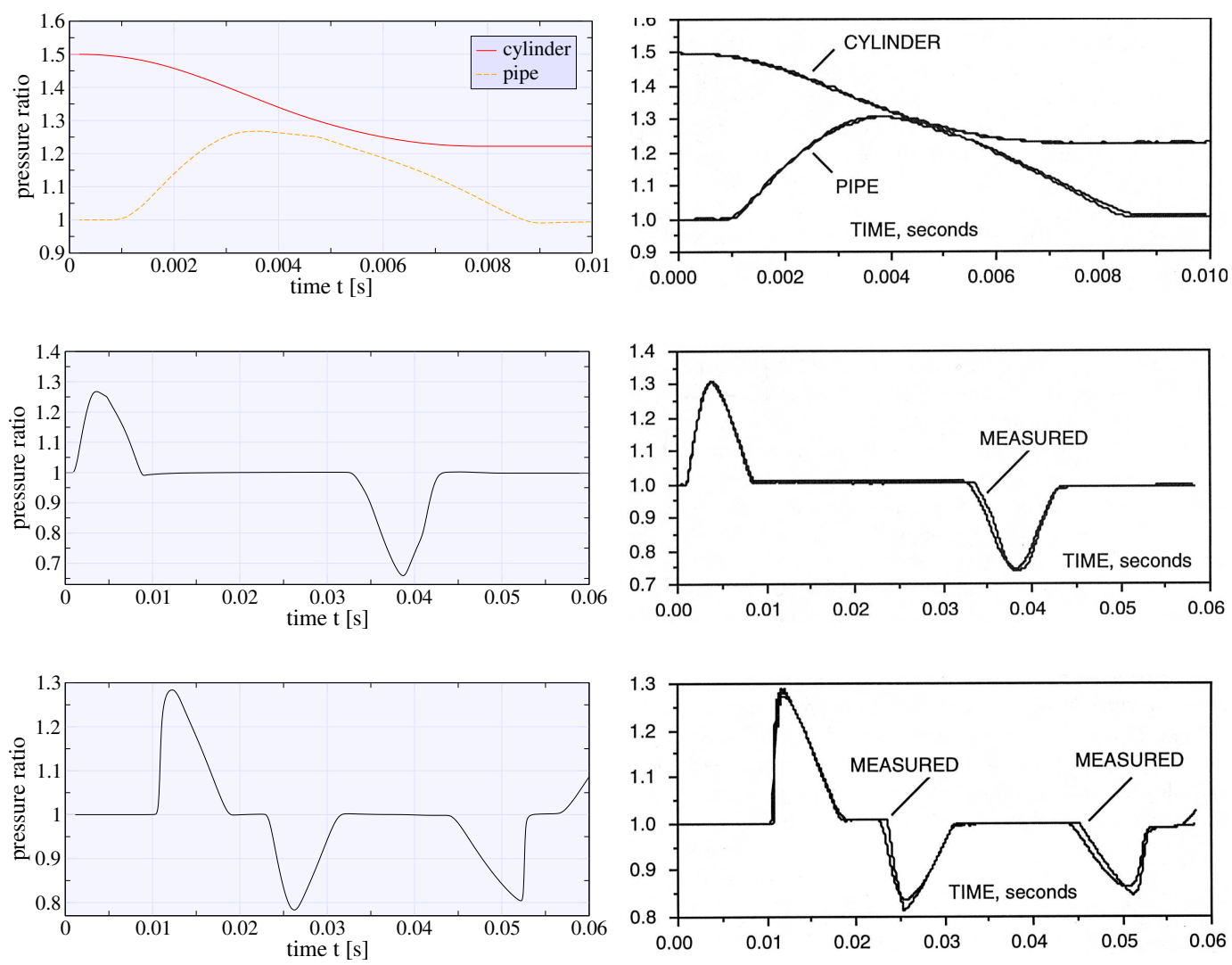

Figure 9.5.4: Exhaust pipe: comparison of the numeric pressure profiles (left) to the experimental ones (right) for the cylinder (top), at the first pressure transducer (middle), and at the second one (bottom).

\section{Setup of the Intake Pipe Experiment}

The same data, as in the above described exhaust pipe experiment, is applied, just the initial pressure of the air in the cylinder is different:

$$
p_{0, \text { cyl }}:=0.8 p_{\text {pipe }}=81060 \mathrm{~Pa}
$$

Thus, an expansion wave is produced by the opening of the connection between cylinder and pipe, simulating an intake pipe at the inlet or transfer ports of a twostroke engine.

\section{Results of the Intake Pipe Experiment}

The same convergence study as in the exhaust pipe experiment has been conducted. The evolution of the expansion wave at measuring point two is displayed in Figure 9.5.5 on the next page. Here too a good convergence is visible.

The main event in this setup is the creation of an expansion wave traveling rightwards (as seen in the graphs in Figure 9.5.6 on page 164). It passes transducer one and later transducer two, is reflected as a compression wave at the open end of 
the pipe, passes point two and one again, is reflected one more time at the now closed valve, superimposes at measuring point one and is clearly visible as an individual wave at transducer two. Also, here a steep fronting of the tail of the expansion wave and the front of the compression wave is clearly visible. Similar to the exhaust pipe experiment the peak value of the reflected pressure wave is slightly underestimated, but the timing of the events is captured very well.

Summarizing the results, a good accordance between physical experiment and simulation has been shown.

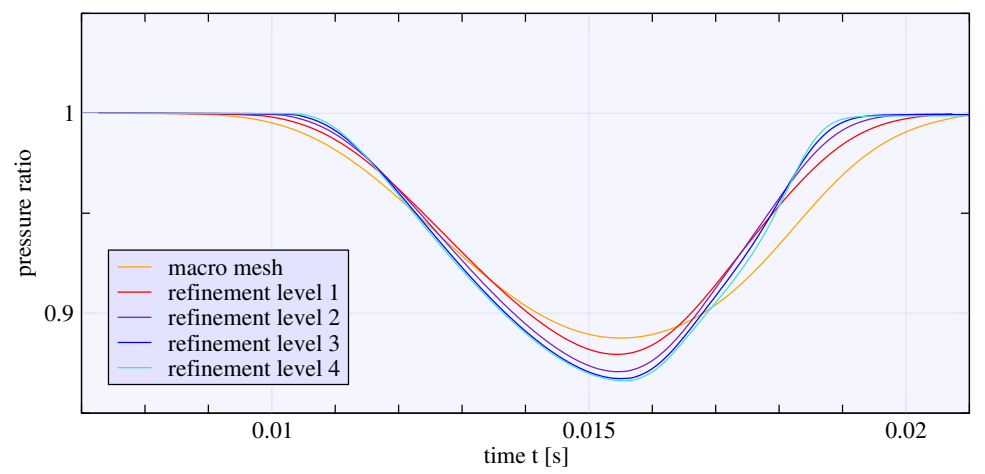

Figure 9.5.5: Intake pipe: convergence on the different meshes of the pressure profile at pressure transducer one for the expansion pulse moving rightwards.

\subsubsection{Temperature-dependent Material Properties: The Otto Cy- cle}

In this test case, the effect of the temperature-dependent material properties, especially the adiabatic exponent $\gamma$, is studied by the compression and expansion process in a closed cylinder (see Figure 9.5.7 on the next page). The calculation of the temperature-dependent heat capacities $c_{p}$ and $c_{\mathrm{V}}$, adiabatic exponent $\gamma$, viscosity $\eta$, and thermal conductivity $\lambda$ is described in Section 3.8 on page 40.

The Otto Cycle has been treated theoretically in Section 2.5.1 on page 20. Apart from the heat release in the combustion process, the Otto Cycle is most importantly influenced by the adiabatic exponent $\gamma$. This exponent determines the compression ratio of the fluid in the cylinder. As the material properties for fresh gas are different from the ones for exhaust air, this has to be taken into account when computing $\gamma$. And also the temperature in the cylinder undergoes drastic changes, which influences $\gamma$ as well.

\section{Setup of the Numerical Experiment}

In order to compare the effect of the temperature-dependent adiabatic exponent $\gamma$, two calculations are conducted: one with constant material properties fixed at 

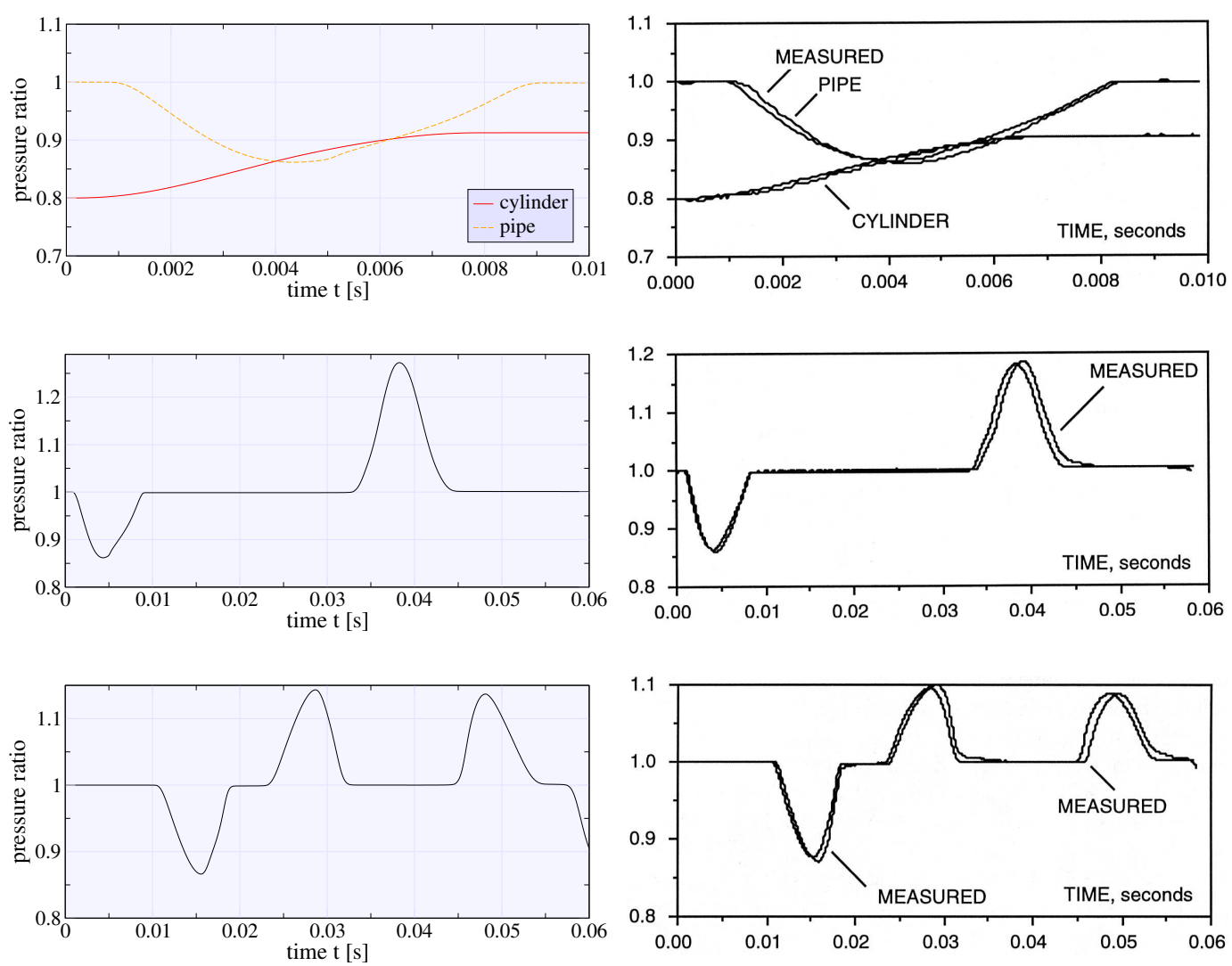

Figure 9.5.6: Intake pipe: comparison of the numeric pressure profiles (left) to the experimental ones (right) for the cylinder (top), at the first pressure transducer (middle), and at the second one (bottom).
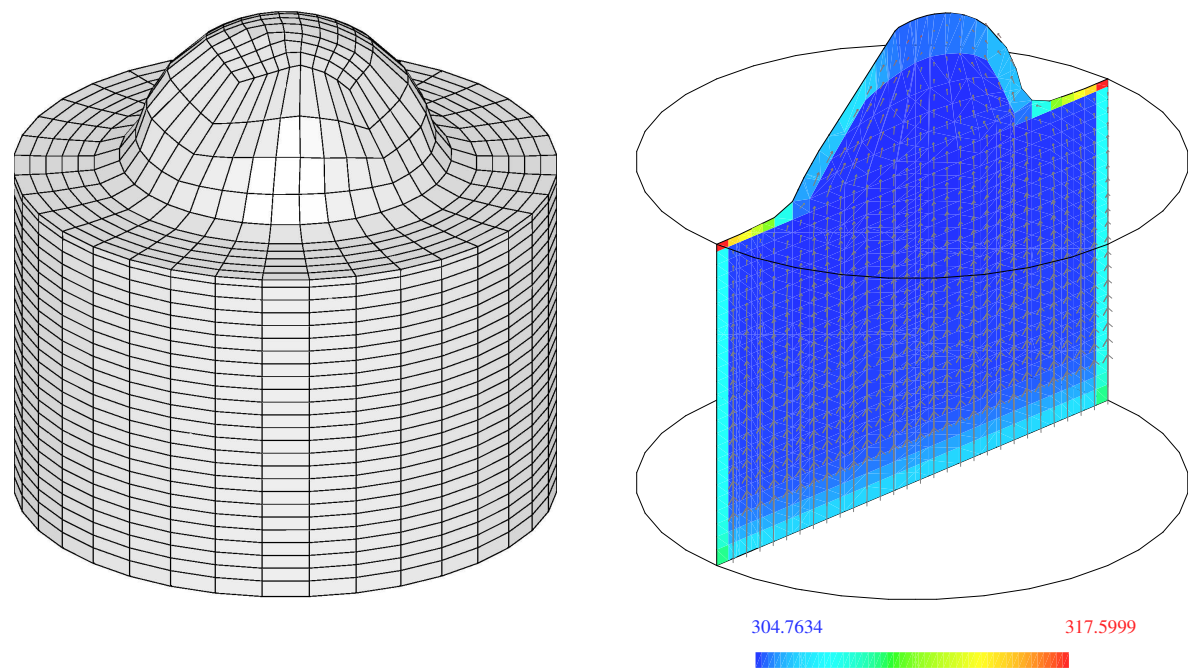

Figure 9.5.7: Otto Cycle: the 10000 element mesh (left) and a clipping plane of the computed temperature with velocity vectors this mesh (right) at crankshaft angle $50^{\circ}$ after bdc. 
$T:=298 \mathrm{~K}$, the other one with temperature-dependent properties (cf. Section 3.8 on page 40). As the calculations are not much influenced on the detailed resolution of the numerical solution of the flow, but rather by the average temperature within the cylinder, the simulation is carried out on a rather coarse mesh with 10000 elements (see Figure 9.5.7 (left)) that is built from CAD data of an existing twostroke engine (cf. Section 10.1 on page 167). Also, the data for the piston motion and the simulation for combustion (see Section 2.6 on page 21) is taken from this real engine (this is described in detail in Section 10.2 on page 170).

The initial conditions are taken to be ambient standard pressure and temperature:

$$
\begin{aligned}
& p_{0}:=101325 \mathrm{~Pa} \\
& T_{0}:=293.15 \mathrm{~K}
\end{aligned}
$$

As there are only wall boundary conditions (see Figure 9.5.8), no inflow or outflow values are needed. The treatment of the (isothermal) wall boundary condition and the moving boundary are explained in detail in Section 4.7 on page 55 .

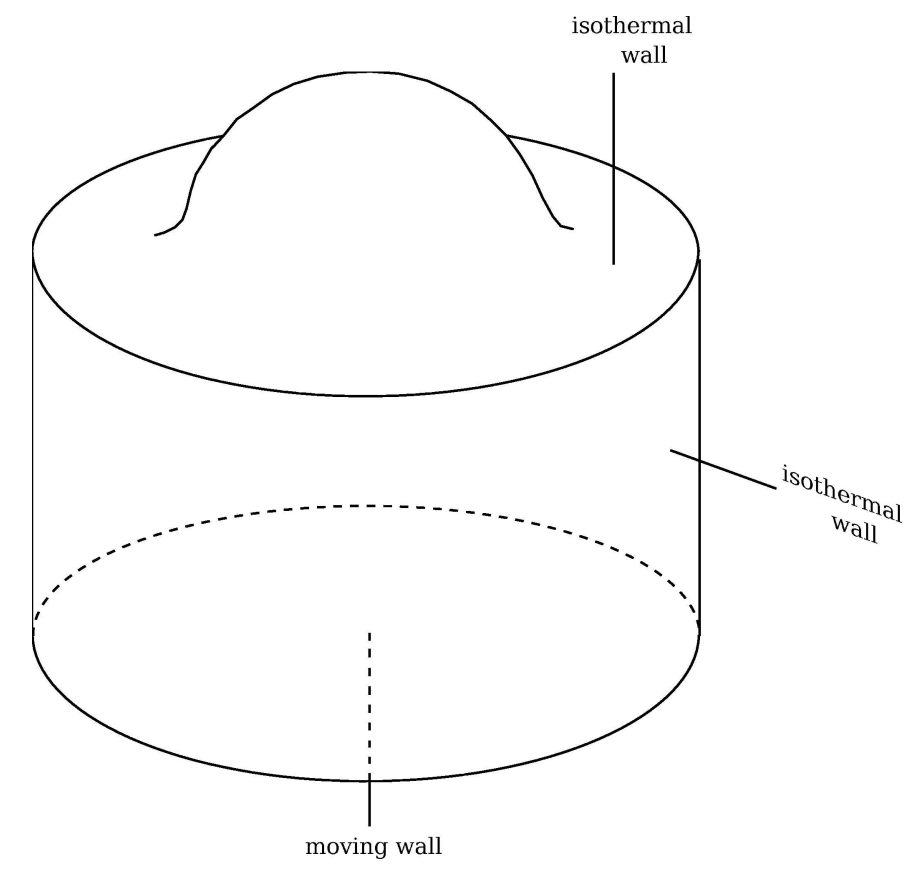

Figure 9.5.8: Otto Cycle: boundary conditions and domain $\Omega$ for the experimental setup.

\section{Results}

In Figure 9.5.9 on the next page the results of the computations are presented. On the left-hand side the compression process is displayed, on the right-hand side the expansion process after the end of combustion at crankshaft angle $50^{\circ}$ after tdc. It is clearly visible that the temperature-dependent adiabatic exponent $\gamma$, whose history 
can be seen in the bottom part of the figure, influences the solution quite strongly. This can be seen best at the end of the expansion process, when in the real twostroke engine the ports would open. These differences in pressure and temperature have a strong effect on the structure of the flow field at the exhaust and scavenging process, as different conditions prevail in the cylinder at port opening.

Therefore, it is very important to imply the temperature dependency of the fluid properties, in order to reach the desired accuracy.
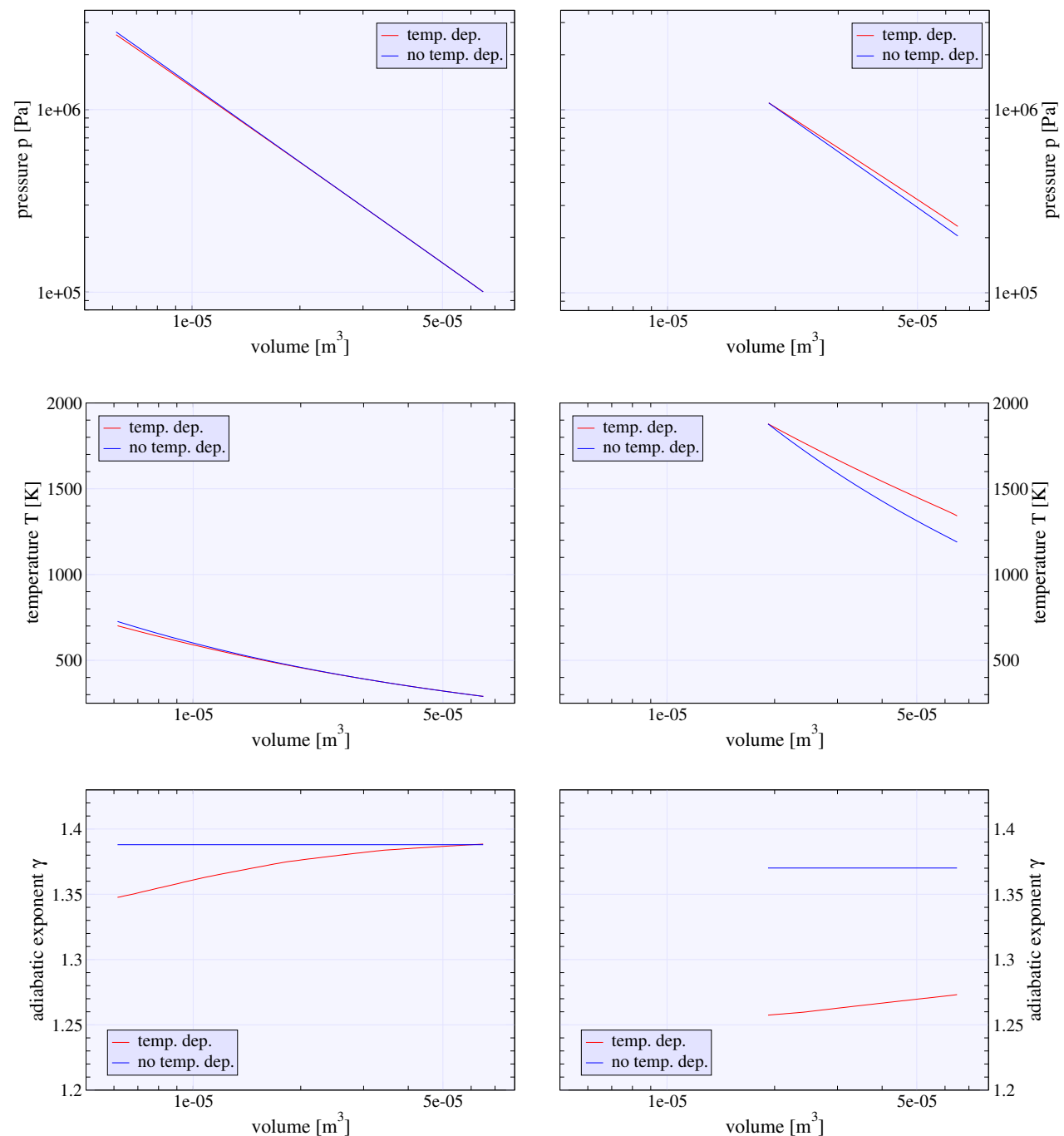

Figure 9.5.9: Otto Cycle: comparison of the pressure $p$ (top), temperature $T$ (middle), and adiabatic exponent $\gamma$ (bottom) development, for the compression (left) and expansion (right) process. 


\section{Chapter 10}

\section{Application: The Two-Stroke Engine}

Our final goal is the simulation of the flow through a two-stroke engine. Apart from the general study of the flow structure in an existing two-stroke engine, we treat in this chapter three problems of particular interest for the engineer. The first one is the question of how much does the crankshaft motion influence the flow situation in the cylinder and the exhaust characteristics. Is it therefore really necessary to simulate the moving parts in the crankcase. The second problem that is illuminated, is an approach to optimize the flow in the cylinder at the scavenging process by employing unsymmetrical transfer ports. And finally, we want to study the mechanism and the paths of the short-circuit flow from transfer port to exhaust port with help of the enhanced fresh-gas tracking (cf. Section 10.11.1 on page 200).

\subsection{Meshes}

\subsubsection{Generation}

The quality of the underlying meshes is of vital importance for the obtained solution. Bad quality meshes may result in a very small time-step (in the case of elements with very small volume) or in an oscillating solution (arising from strongly skewed elements). It was pointed out in Remark 4.2.5 on page 47 that our meshes consist of hexahedrons due to better performance. As we are in the case of a complex real-world mesh based on CAD data, a commercial mesh generator is necessary with this type of mesh (as e.g. [Ice04]).

The geometry of a two-stroke engine has a complex topology, thus it is inevitable to use a composition of a family of meshes assembled by the grid merging method to form the whole geometry (as shown in Figure 10.1.1 on the following page). It would not be possible to construct one single mesh meeting all the geometrical restraints. With the grid merging technique, O-grids can be used to mesh the inlet, transfer port and outlet duct, thus accounting for their cylindrical shape and the possible boundary refinement (see the upper left-hand side of Figure 10.1.2 on page 169). Also, the upper edge of the transfer ports connecting to the cylinder does not need to be horizontal (see Figure 10.1.2 (bottom)), as would be the case without grid 
merging due to the horizontal layering of the cylinder elements, as needed by the snapper algorithm (cf. Section 5.2 on page 72).

But also with grid merging, a problem in mesh generation arises. The lower part of the piston walls that closes the inlet and outlet ports, reaches far downwards into the crankcase at bdc (see Figure 10.1.2 (upper right)). These parts are essential for the timing of the inlet port, therefore, they cannot be shortened. A meshing of such a situation is difficult but feasible. The resulting elements are not very regular but within the limits of volume and skew.

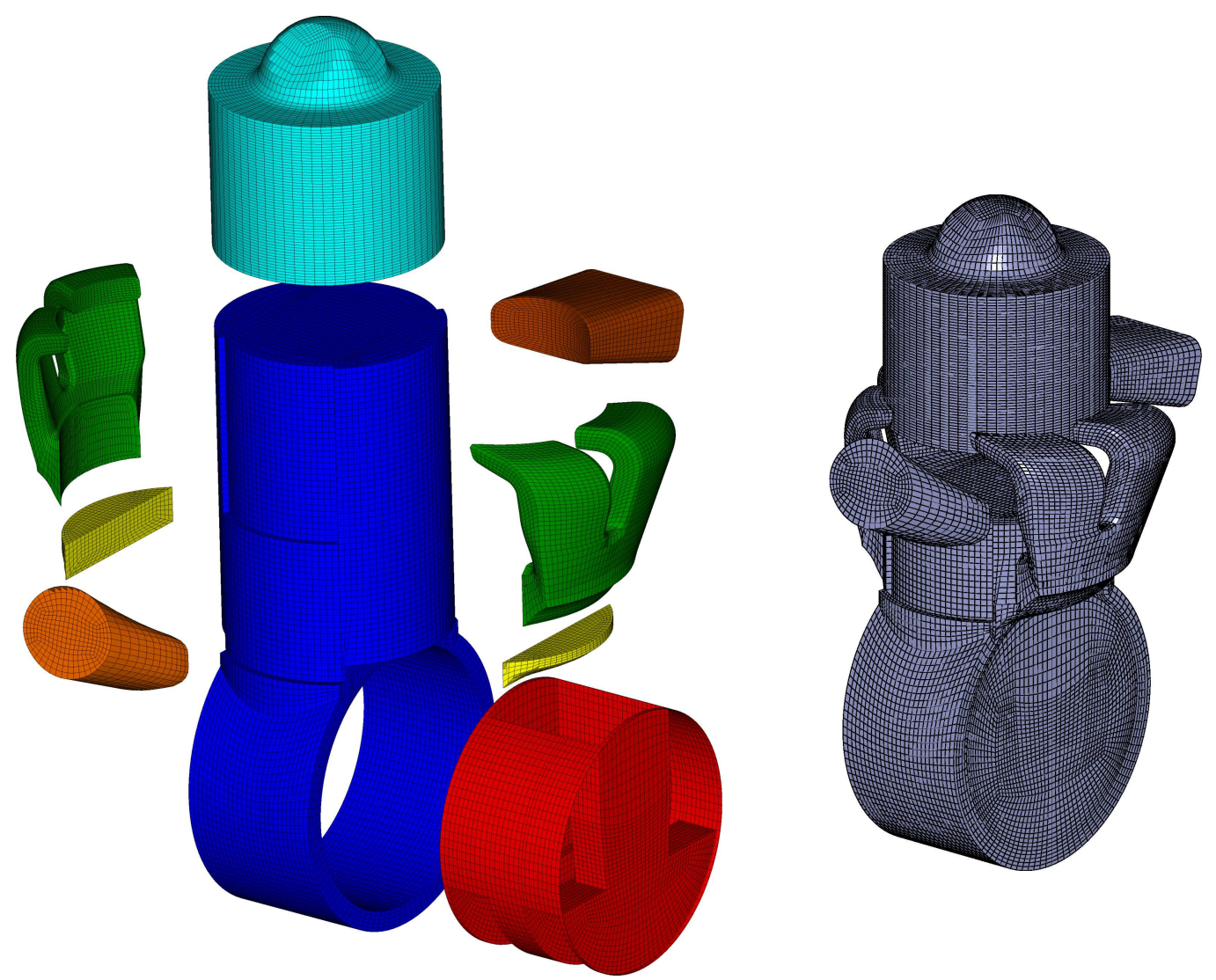

Figure 10.1.1: Meshes: the complete mesh is assembled by several different parts (left), on the right the 250000 element mesh is displayed.

\subsubsection{Different Size Meshes}

In order to check for the mesh dependency of our solution and to speed up convergence by the enhanced initial data method according to Section 6.3 on page 97, we need a whole series of families of meshes. Seven families of meshes have been generated. The element numbers can be seen in Table 10.1 on page 170. Further meshes with higher element numbers are created by global or adaptive refinement 

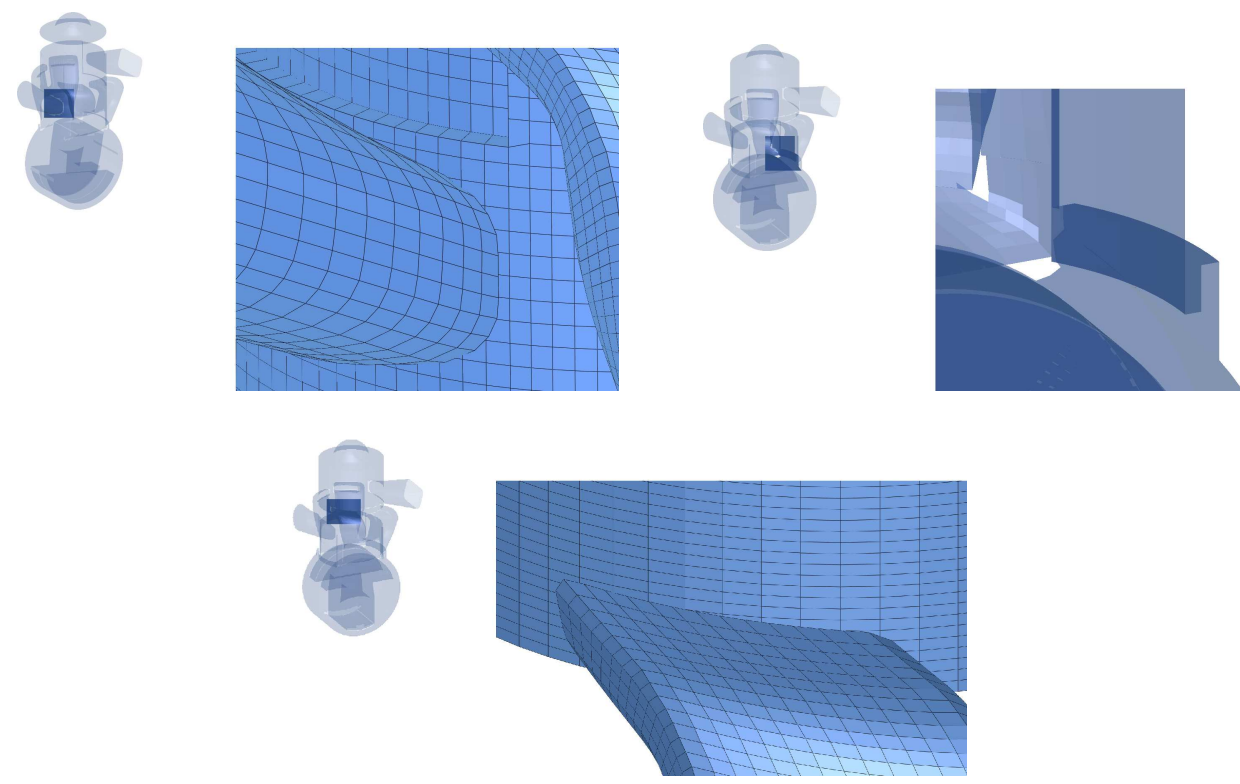

Figure 10.1.2: Meshes: details of the mesh generation, inlet-crankcase connection (upper left), piston wall reaching down the crankcase (upper right), and transfer port-cylinder connection (bottom).

of the existing ones. Therefore, the number of elements of a family of meshes is only limited by the computational time of the simulation on the resulting mesh.

\subsubsection{The Silencer-An Alternative to a Transparent Boundary Condition}

We have seen in test case 9.5.1 on page 158 that the handling of the outflow boundary is not easy. The straightforward characteristics based approach reflects waves back into the interior of the domain (if we are in the subsonic regime, which is the case in most of the outflow duct).

One method to cover this problem is the usage of a transparent or artificial boundary condition $(\mathrm{ABC})$ at the end of the outflow duct. These boundary conditions approximate the flow that would continue in an infinitely long outflow duct. They are an improvement to the characteristics based boundary treatment in that they do not influence the interior flow by perturbations created at the boundary. Usually, this kind of boundary condition is found in the simulation of outer flow problems, e.g. airfoil calculations (cf. [Tre00]). In our case of the viscous threedimensional time-dependent duct flow with boundary layer, the transparent boundary condition approach is not as well studied as the far field boundary in an outer flow problem. But, more importantly, in the two-stroke engine context the pressure wave, resulting from exhaust port opening, is reflected at the transition of outflow duct to silencer. Therefore, in reality we have a strong interaction between outflow boundary and interior flow. This can only be simulated by adding the silencer to our family of meshes and thus calculating the flow through this part of the engine as well. The disturbing boundary is now far away from the cylinder at the outlet of 
the silencer. As can be seen from Table 10.1, only about $8 \%$ of the total number of elements are needed for the mesh of the silencer due to its basic geometry.

Table 10.1: The element number of the different mesh families for the twostroke engine simulation

\begin{tabular}{|cccccc|}
\hline mesh family & 15,000 & 30,000 & 60,000 & 120,000 & 250,000 \\
\hline \hline inlet duct & 270 & 432 & 826 & 1,680 & 3,456 \\
crankcase & 3,952 & 7,595 & 14,444 & 31,572 & 65,838 \\
crankshaft & 1,920 & 3,420 & 7,916 & 14,313 & 26,848 \\
lower transfer ports & 236 & 430 & 866 & 1,744 & 3,440 \\
transfer ports & 2,144 & 4,272 & 8,032 & 17,522 & 34,048 \\
cylinder & 5,271 & 10,206 & 20,922 & 41,300 & 82,548 \\
outlet duct & 512 & 1,298 & 2,632 & 5,032 & 10,384 \\
silencer & 1,160 & 2,592 & 5,190 & 10,507 & 20,736 \\
\hline
\end{tabular}

\subsection{Configuration}

\subsubsection{Geometrical Data}

The geometrical data of the two-stroke engine with which we test our software are given in Table 10.2 on the next page (for the definitions of these entities see Section 2.3 on page 11). The values are extracted from the CAD data of a real engine employed in a chain saw. The port areas as functions of time of the different ports are shown in Figure 10.2.1 on the next page. The timing for the opening and closing is specified in Table 10.3 on the facing page. The piston motion is determined by the formulae for $h_{p}$ and $v_{p}$ stated in Lemma 2.3.10 on page 13. The rotation of the crankshaft is given by the value for the rotations per minute. Thus, in our case we have $9000 \mathrm{rpm}$, i.e. the crankshaft rotates $360^{\circ}$ per $\frac{1}{150 \mathrm{~s}}$.

As described in Chapter 8 on page 107, the connecting rod is neglected within our simulation.

\subsubsection{Temperature-dependent Material Properties}

In order to calculate the material properties of the fresh gas and the exhaust gas, the formulae from Section 3.8 on page 40 with the values from the Tables B.2 on page 209 and B.4 on page 210 in Appendix B on page 209 are used. There the 
Table 10.2: Geometrical data of the test engine

\begin{tabular}{|cc|}
\hline variable & value \\
\hline \hline bore $d_{\mathrm{b}}$ & $47.0 \mathrm{~mm}$ \\
stroke $l_{\mathrm{s}}$ & $34.0 \mathrm{~mm}$ \\
connecting rod length $l_{\mathrm{cr}}$ & $58.0 \mathrm{~mm}$ \\
trapped stroke $l_{\mathrm{ts}}$ & $23.3 \mathrm{~mm}$ \\
swept volume $V_{\mathrm{s}}$ & $59.0 \mathrm{~cm}^{3}$ \\
trapped swept volume $V_{\mathrm{ts}}$ & $40.4 \mathrm{~cm}^{3}$ \\
rotations per minute $r p m$ & $9000 \frac{1}{\mathrm{~min}^{2}}$ \\
crankcase compression ratio $C R_{\mathrm{cc}}$ & 1.35 \\
geometric compression ratio $C R_{\mathrm{g}}$ & 10.59 \\
trapped compression ratio $C R_{\mathrm{t}}$ & 7.57 \\
\hline
\end{tabular}

Table 10.3: Port timing for the test engine

\begin{tabular}{|ccc|}
\hline port & $\begin{array}{c}\text { opens } \\
\text { crankshaft atdc }\end{array}$ & $\begin{array}{c}\text { closes } \\
\text { crankshaft atdc }\end{array}$ \\
\hline \hline inlet port & 285 & 75 \\
main transfer port & 130 & 230 \\
auxiliary transfer port & 130 & 230 \\
outlet port & 104 & 256 \\
\hline
\end{tabular}

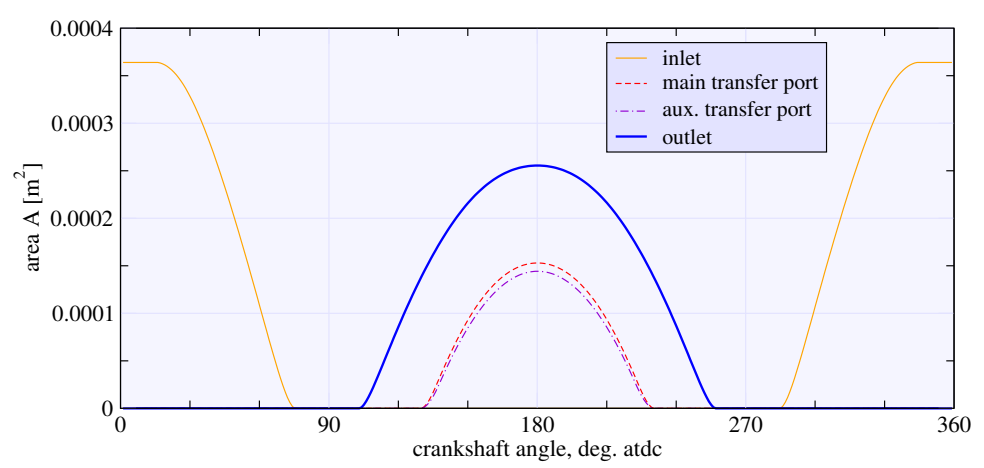

Figure 10.2.1: Port areas by piston control of the different ports. 
values for fresh air are given explicitly whereas the exhaust gas is assumed to have the composition as stated in Paragraph 2.6.3 on page 22.

\subsubsection{Combustion Data}

In Section 2.6 on page 21 it was specified how the combustion process is modeled. As the combustion process itself is not simulated, we need the actual state of the exhaust gas within the cylinder after the combustion has terminated. This data is extracted from an experimental measurement:

$$
p(\mathbf{x}, t):=1092360 \mathrm{~Pa} \quad \text { for } t=50^{\circ} \text { crankshaft angle atdc, } \mathbf{x} \in \text { cylinder }
$$

The density does not change during combustion, therefore, the temperature can be calculated by the thermal equation of state (3.5.1).

\subsection{Initial and Boundary Data}

\subsubsection{Initial Conditions}

The initial data for the coarsest mesh is set to ambient standard temperature and pressure for the inlet and crankcase:

$$
\begin{aligned}
& \rho_{0}:=1.2039 \frac{\mathrm{kg}}{\mathrm{m}^{3}} \\
& p_{0}:=101325 \mathrm{~Pa}
\end{aligned}
$$

and for the outlet and silencer:

$$
\begin{aligned}
& \rho_{0}:=0.4100 \frac{\mathrm{kg}}{\mathrm{m}^{3}} \\
& p_{0}:=101325 \mathrm{~Pa}
\end{aligned}
$$

Whereas in the cylinder the trapped compression ratio is applied to these values based on a polytropic compression:

$$
\begin{aligned}
& \rho_{0}^{\text {cyl }}:=\rho_{0} C R_{\mathrm{t}}=9.1135 \frac{\mathrm{kg}}{\mathrm{m}^{3}} \\
& p_{0}^{\text {cyl }}:=p_{0}\left(C R_{\mathrm{t}}\right)^{1.4}=1723657 \mathrm{~Pa}
\end{aligned}
$$

The velocity field is assumed to vanish in the whole geometry. It was explained in Section 6.3 on page 97 that the initial data does not influence the asymptotic (in the sense of (6.3.1)) solution. The boundary conditions determine the converged periodic solution. The initial values just influence the time needed for the solution to reach a converged state.

For the subsequent meshes, the converged solution of the previous mesh is used as initial data in order to speed up the convergence (as described in detail in Section 6.3 on page 97 ). 


\subsubsection{Boundary Conditions}

The numerical treatment of the boundary conditions is described in Section 4.7 on page 55. The outflow boundary condition has also been discussed in Paragraph 10.1.3 on page 169. The inflow boundary is the only one that needs further consideration. Two possible approaches are feasible to cover this boundary. If the massflow data from a physical experiment is available, this data can be used to impose a massflow condition at the inflow boundary. Otherwise, a the ambient standard pressure $p_{\text {in }}:=$ $p_{0}$ and density $\rho_{\text {in }}:=\rho_{0}$ can be prescribed.

The advantage of the massflow condition is a better comparability of two different engine geometries. Because the massflow through the engine is always the same (due to the setting at the inflow boundary), the delivery ratio DR is kept constant, and therefore also the scavenge ratio SR (with the assumption of identical cylinder volumes). Therefore, the trapping efficiency TE as main criterion is only influenced by the geometrical shape of the engine, and not by the amount of delivered fresh gas.

The advantage of the standard inflow condition, on the other hand, is its ease of use. No time-dependent measurements on a real engine need to be conducted, and no time-dependent massflow value needs to be enforced at the inflow boundary. But an interaction of the flow with this kind of boundary condition results in a variable massflow rate, which makes it difficult to compare different geometries.

\subsection{Parallelization}

The code was executed on IBM Regatta p650 and p690+ with up to 32 Power $4+$ processors with $1.7 \mathrm{GHz}$ in shared memory operation.

\subsubsection{Partitioning}

As stated in Chapter 7 on page 101, the parallelization was performed using the OpenMP standard for shared memory parallel architectures. The concept of multiple simultaneous partitions of the same mesh, explained in Section 7.4 on page 104, relies on the shared memory concept. In Figure 10.4 .1 on the following page the $\Delta t$-level distribution in a 250000 element mesh at $110^{\circ}$ crank angle atdc (top) with two $\Delta t$-level dependent partitionings (level 1 (middle left) and level 5 (middle right)) is shown. The bottom row of the figure displays the resulting critical elements at the interfaces of two partitioning domains. On those elements a race condition can occur, therefore, the element has to be locked while its contents are manipulated by a process (see also Paragraph 7.3.1 on page 103).

\subsubsection{Performance Analysis}

Two types of studies can be conducted. One is to increase the number of processors for the same problem. Thus, by doubling the number of processors, the size of the problem per processor is halved. This approach leads to the concept of speed-up and efficiency (see Section 7.1 on page 101). The other type consists in increasing 

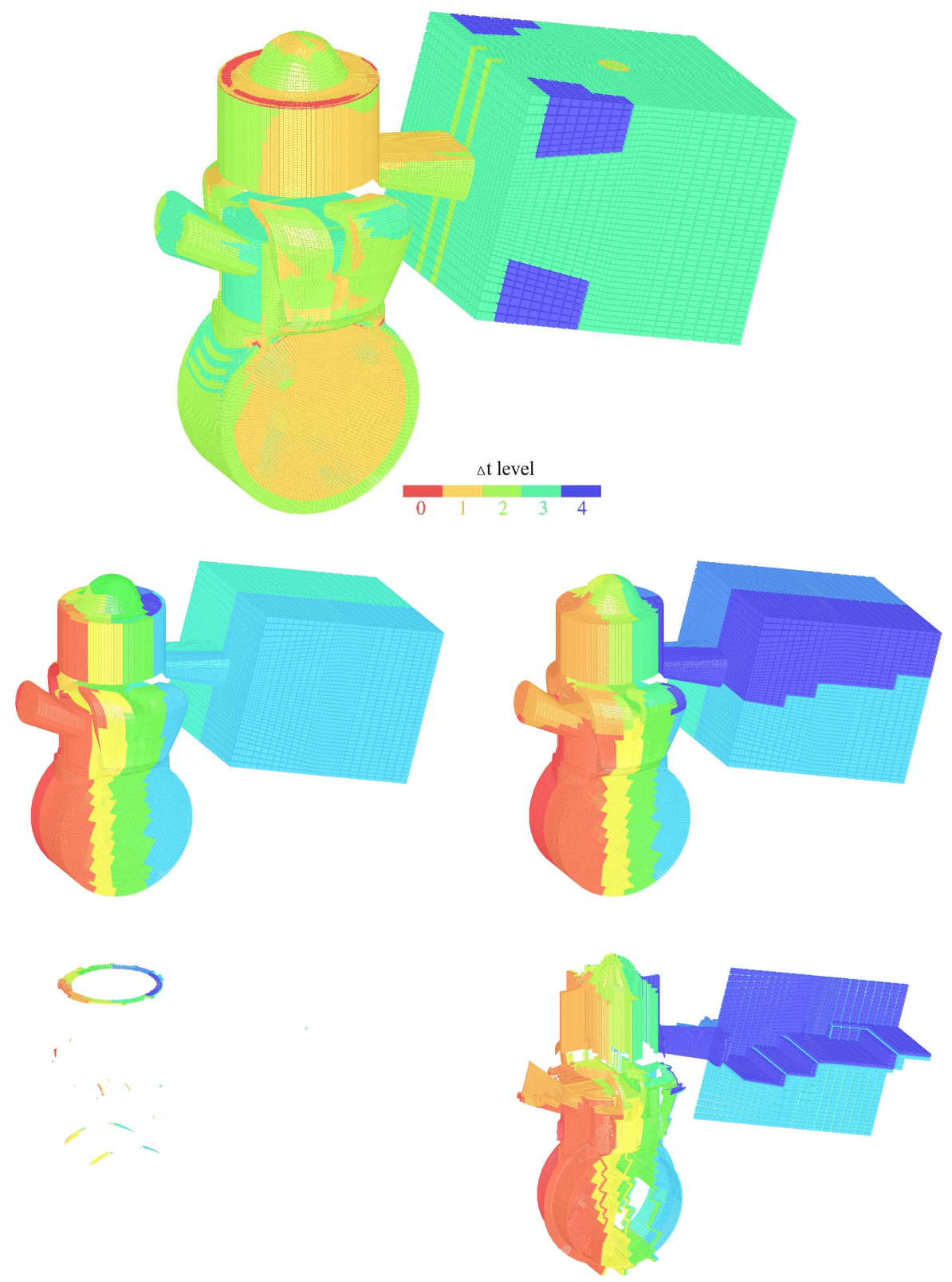

Figure 10.4.1: Parallelization: For a given local time-step level distribution (top) the mesh is for each $\Delta t$-level partitioned such that the number of elements belonging to that level is evenly distributed to the single processors (middle left for level 0 and middle right for level 5). The interface elements between two partitioning domains belonging to the respective $\Delta t$-level are shown in the bottom row (depicted is the 250000 element mesh at $110^{\circ} \mathrm{crank}$ angle atdc with maximum $\Delta t$-level $l_{\max }:=5$ and 16 partitioning domains). 
the problem according to the number of processors such that the problem size per processor remains constant. This is measured by the scalability (as defined in Section 7.1 on page 101). Both types of studies have been performed. The results can be seen in Figure 10.4.2. The speed-up (upper left) and the associated efficiency (upper right) for the 1 million and 4 million element mesh is quite good for such a complex algorithm. With 8 processors the larger mesh performs slightly better. This trend continues probably on more processors. The scalability (bottom) is based on simulations on 500000, 1 million, 2 million, and 4 million elements meshes with $1,2,4$, and 8 processors respectively. Also, here a relatively good performance is demonstrated.
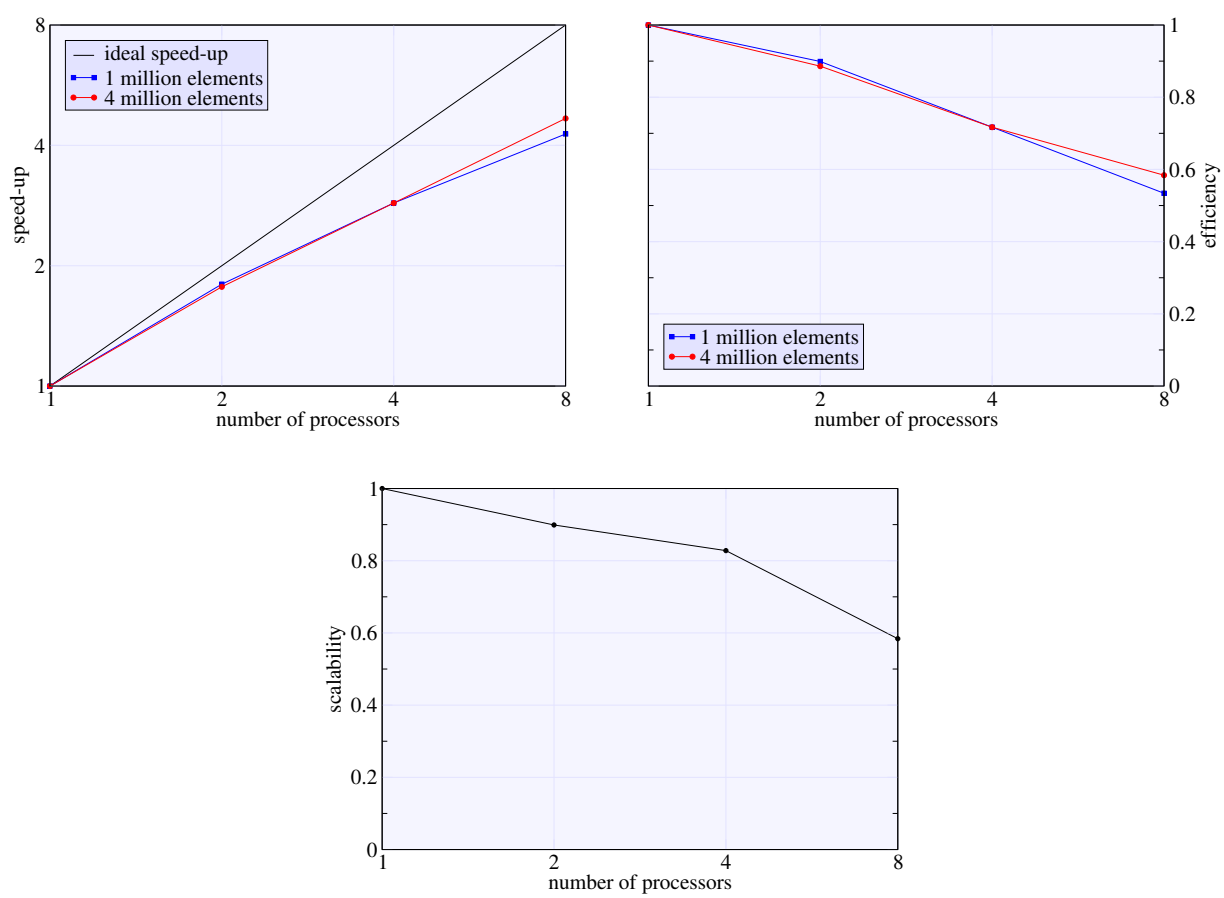

Figure 10.4.2: Parallelization: speed-up (upper left) and associated efficiency (upper right) for the simulations on the 1 million and 4 million element meshes and the scalability (bottom) for a computation on the 500000 (1 processor), 1 million (2 processors), 2 million (4 processors), and 4 million (8 processors) element meshes.

\subsection{Visualization and Characteristical Diagrams}

The visualization of large data is a very important issue in analyzing the flow through a complex geometry. The visualization software, that is part of our software package, is based on the library GRAPE (for GRAphics Programming Environment) (see [Gra99, BPR01, NORS97, OR97, OR99, RS95, WR92]) and provides an interface for our mesh structures. The visualization software reuses large parts of the code of the main simulation software, e.g. to move the piston and crankshaft and to calculate the grid merging. Additionally, several visualization routines have been 
implemented to display certain aspects of the special algorithms used in the simulation. These include the visualization of the local time-step levels (already seen in the upper part of Figure 10.4.1 on page 174), the partitioning for the parallel algorithm (the same figure in the middle row) with the ghost cells (on the bottom row of the figure), and the opaque clipping routine to enhance the clarity of the presentation. Already present in GRAPE is a wide variety of display methods, e.g. the level display method (as seen in Figures 10.6.7 on page 182 to 10.6.9 on page 184), the particle trace method (in Figure 10.6.5 on page 181 and 10.6.6 on page 181), and multiple-isoline and vector field clipping displays (e.g. Figure 10.6.11 on page 187 and 10.6.20 on page 193). All of these routines can be used in a time-dependent context, thus taking into account the variations in the flow with time (compare also the presentation of the results in the next Section 10.6). With the help of these very powerful routines an exhaustive analysis of the flow structure in the two-stroke engine is made possible.

Furthermore, for the quantitative study of the exhaust characteristics of the engine several output files are created during the simulation. This data can be used to generate, e.g. massflow and trapping efficiency diagrams (as displayed e.g. in Figures 10.6.14 on page 189 and 10.6.13 on page 189). In this manner the quality of two or more geometries can be compared with each other.

\subsection{Results}

\subsubsection{Convergence in Time}

In Section 6.3 on page 97 it was explained what is meant by convergence in time. The difference of the numerical solution, measured in a suitable norm, between two consecutive rotations vanishes. This has to be verified within the simulation. We continue the calculation as long as the influence of the initial data is still above a certain threshold. As the scavenge ratio SR and the trapping efficiency TE are the main characteristic values to assess the engine geometry, the difference of the SR and the TE of two consecutive rotations is taken as indicator for a convergence in time. The convergence history for the different geometries is displayed in Figure 10.6.1. It can be seen very clearly that only on the first mesh the solution starts far away from the asymptotic state. On the finer meshes the enhanced initial data approach accelerates the convergence to such an extent that the calculation can be terminated after no more than three periods. The difference after these three periods is for the scavenge ratio less than $1 \%$ (absolute difference of 0.431 from 49.406 ) and even less than $0.5 \%$ for the trapping efficiency (an absolute difference of 0.332 from 75.927 ).

\subsubsection{Convergence for Mesh Width $h \rightarrow 0$}

When the convergence in time is assured, the calculated numerical solution can be checked for mesh dependency. This is done by comparing the solutions from different meshes with each other. Here the isoline images on the meshes as well as the scavenge ratio SR-trapping efficiency TE graph are taken as indicators for convergence. As the mesh width $h$ decreases, the solutions, and consequently the 

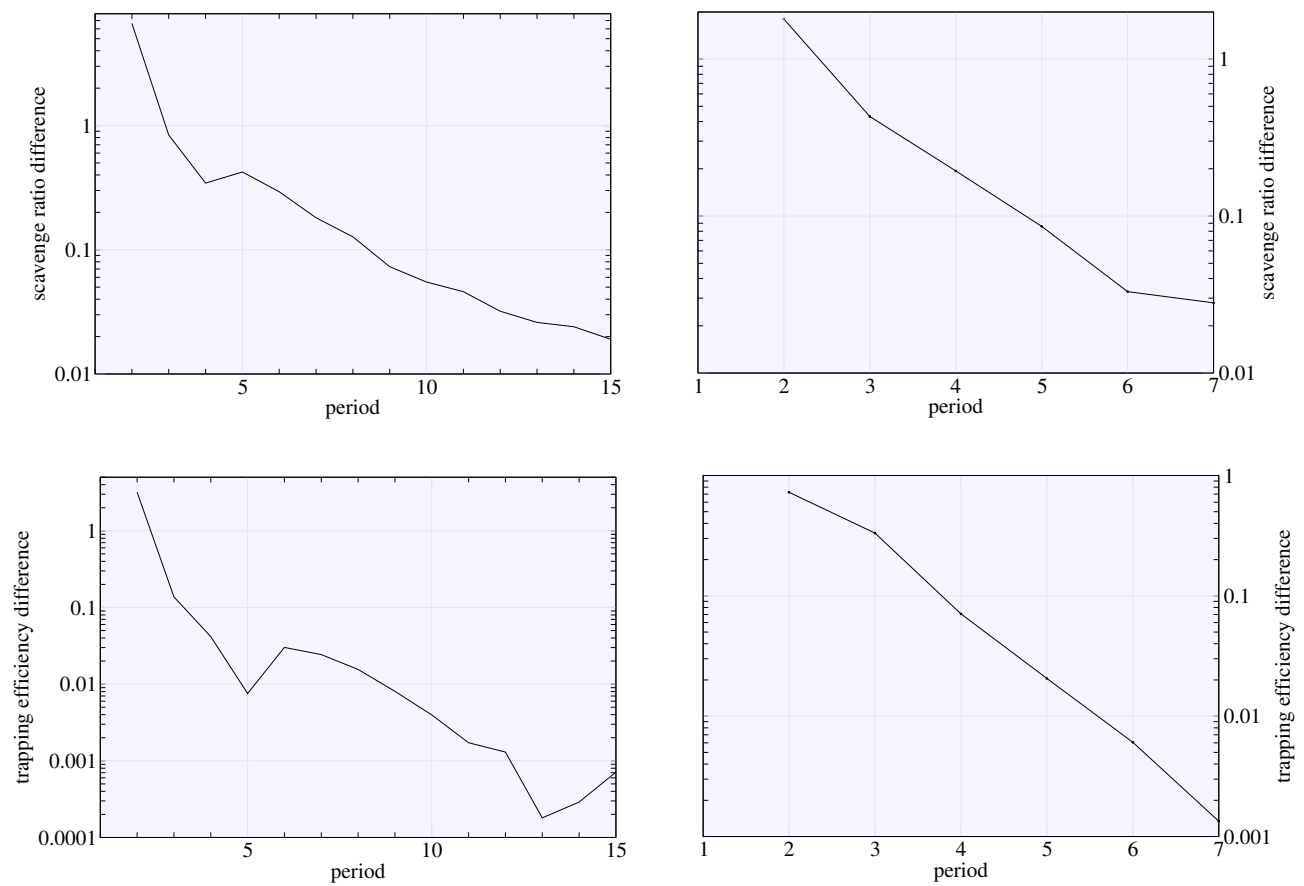

Figure 10.6.1: The temporal convergence of the scavenge ratio (top) and trapping efficiency (below) on the 15000 element mesh without enhanced initial data (left) and on the 120000 element mesh with enhanced initial data (right). Displayed is the difference of the scavenge ratio and the trapping efficiency between two consecutive rotations. 
associated SR-TE graphs, should converge to a mesh independent solution. This is studied for the flow structure of our simulation in Figure 10.6.2. The flow pattern of the solution on the coarse meshes is clearly distinct from the one on the finer meshes. The loop structure is visible only with the finest resolutions. Although there are still differences in details of the looping structure, the general flow pattern converges on the finest meshes. This sequence of images demonstrates very impressively how important it is to calculate a solution on several different size meshes. For an analysis of the flow it is indispensable to know how much influence of the mesh resolution is still present in the numerical solution.

In Figure 10.6.3 on the next page the effect of this converging flow structure is shown in the SR-TE diagram. The convergence for the scavenge ratio SR is clearly visible even on the coarser meshes from 120000 elements onwards. This is not surprising, because we force the delivery ratio DR to a fixed value due to the massflow condition at the inflow boundary. The convergence for the trapping efficiency TE starts to show on the finest meshes. Thus, the trapping efficiency is also assumed to not change much any more on higher resolutions.(For an in depth analysis of the characteristical diagrams see Paragraph 10.6.5.)
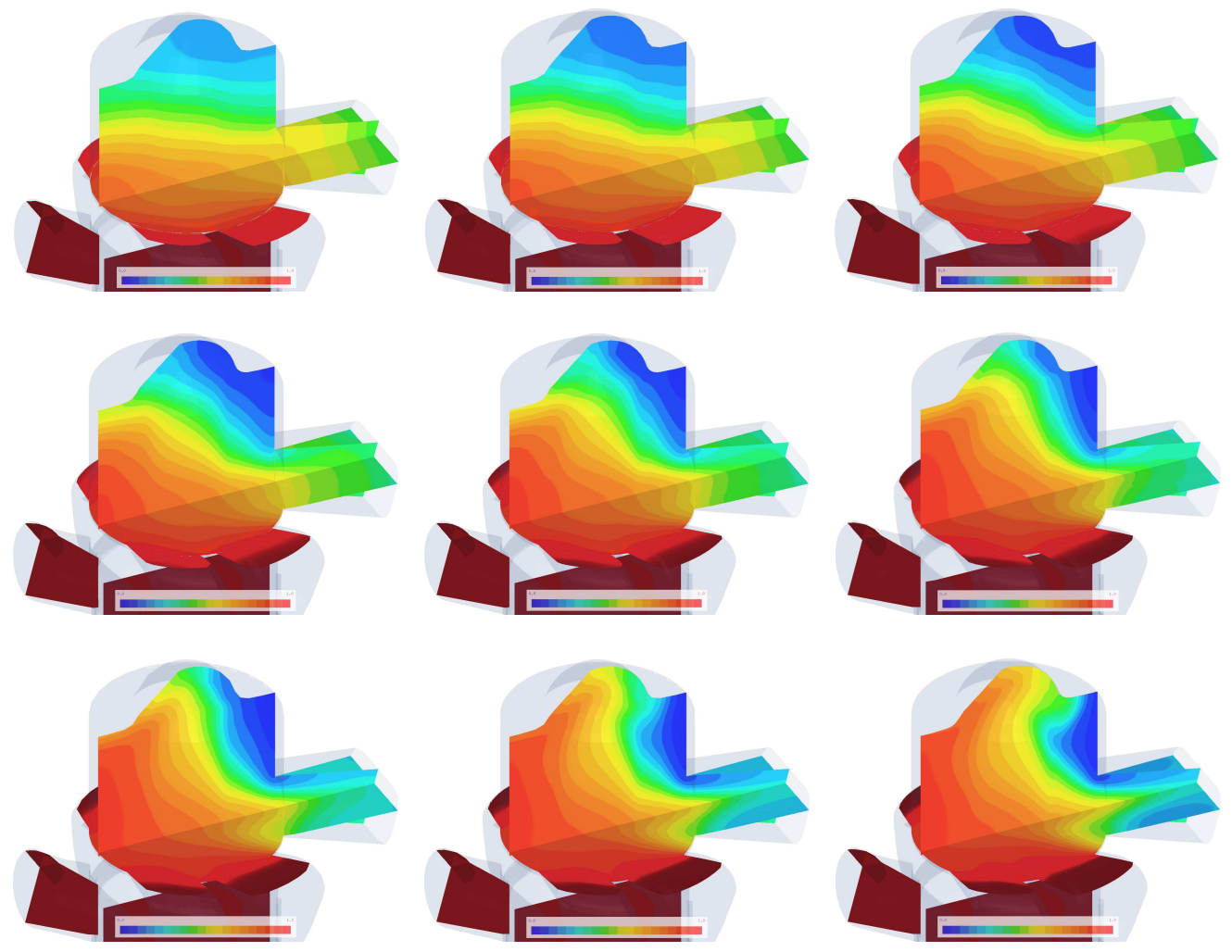

Figure 10.6.2: Convergence of the flow structure for the meshes from 15000 to 4000000 elements (line-by-line) at $200^{\circ}$ crank angle atdc. 


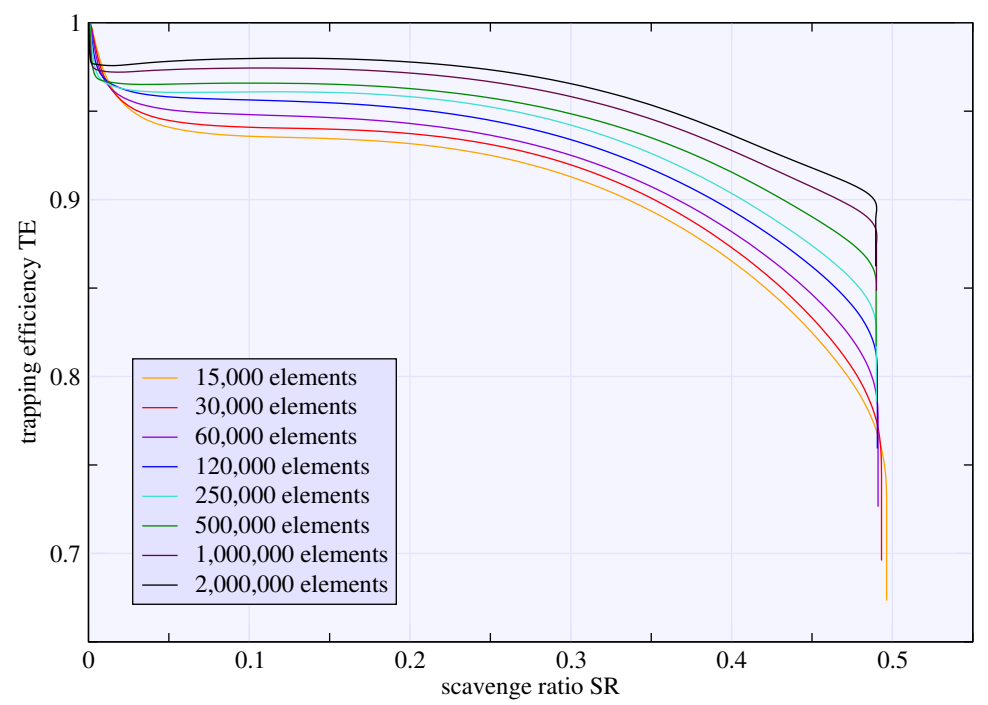

Figure 10.6.3: Convergence of the scavenge ratio-trapping efficiency graph depending on the mesh size.

\subsubsection{Comparison with Measured Data}

As we are simulating an existing two-stroke engine, experimental measurements are available. The simulated pressure in the cylinder during the scavenging process and the simulated pressure in the crankcase during the whole period are compared to this experimental data. The result is shown in Figure 10.6.4 on the following page. As the numerical pressure is an average from the whole cylinder and the whole crankcase respectively, the plot is smoother than the measured data that is gathered at one point. But the otherwise very good agreement of this data confirms the high quality of our numerical simulation.

\subsubsection{Visualization of the Flow Structure}

The analysis of the large data sets of many million numbers calls for powerful visualization strategies. With our visualization software many time-dependent display methods are at hand to study the flow structure of the scavenging process.

First, the main problem of two-stroke engines, the short circuiting, can be demonstrated quite impressively by the particle trace method shown in Figure 10.6.5 on page 181. Here, the blue particles represent the exhaust gas, the orange and red ones the fresh gas. The dark blue exhaust particle stays in the cylinder during the next combustion, but more importantly, the red fresh gas particle is not trapped in the cylinder but leaves through the exhaust port before it is closed by the upwards moving piston. In Figure 10.6.6, the intake flow into the crankcase is visualized. The light green particles start at the opening of the inlet port at $285^{\circ}$ crank angle atdc, and at tdc they are already in the upper part of the crankcase (Figure 10.6.6 (left)). The dark green particles are released at tdc and are pulled into the lower part of the crankcase by the suction of the crank shaft, whereas the earlier particles 

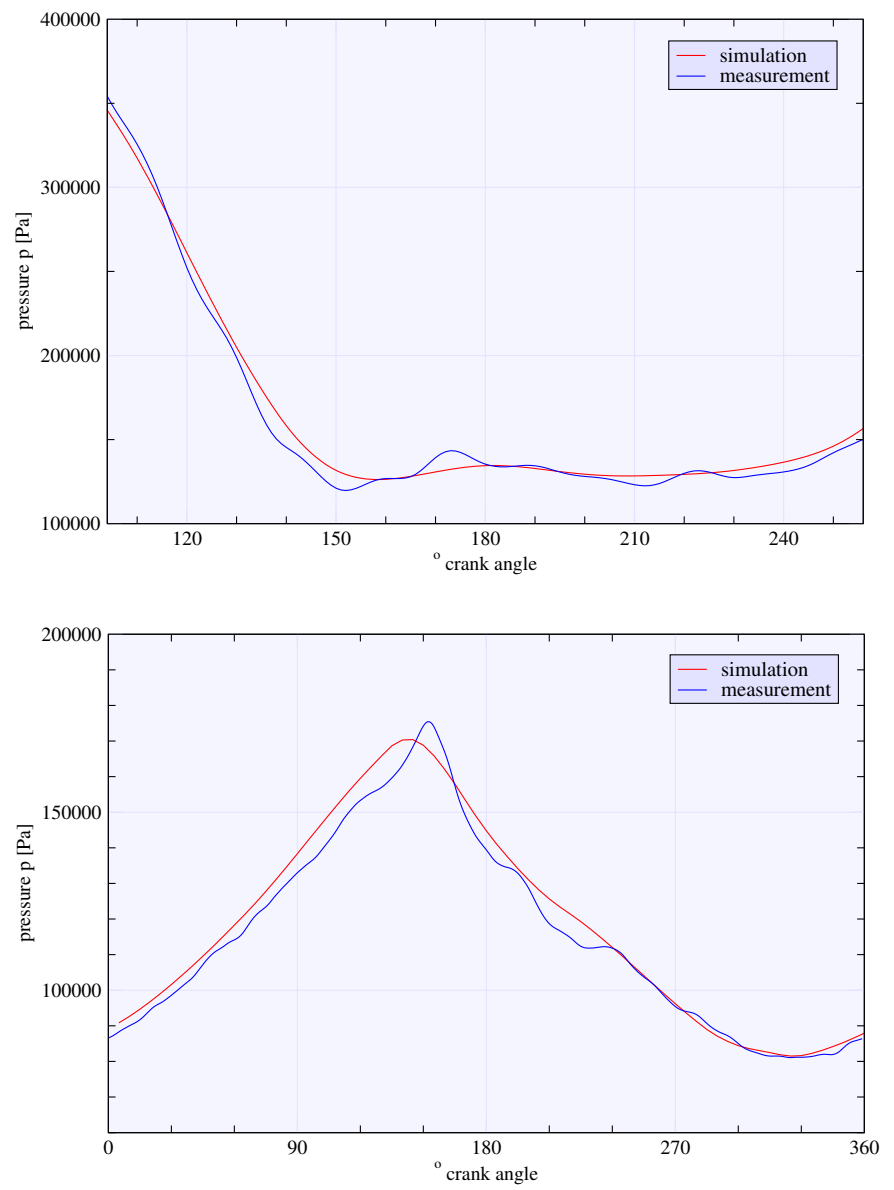

Figure 10.6.4: Comparison with measured data: cylinder pressure during the scavenging process (top) and crankcase pressure (bottom). 
stay in the upper crankcase (Figure 10.6.6 (middle and right)).
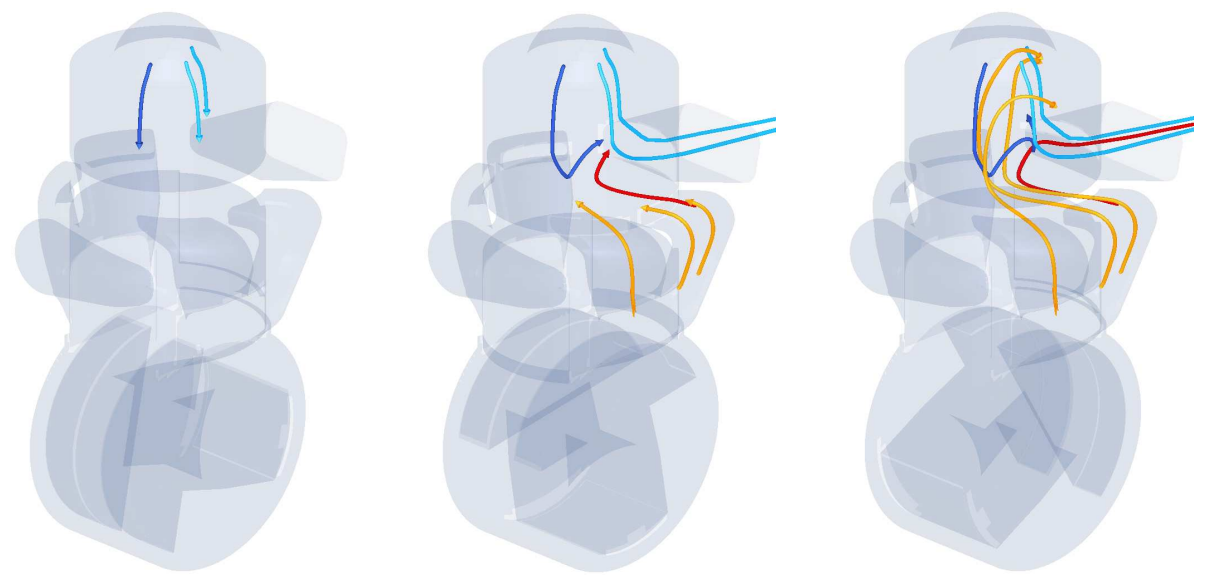

Figure 10.6.5: Visualization of the flow structure: Particle traces at $100^{\circ}$ (left), $170^{\circ}$ (middle), and $240^{\circ}$ crankshaft angle atdc (right). Exhaust gas particles (blue) and fresh gas particles (orange/red) on their way through the engine, the dark blue exhaust particle does not leave the cylinder, the red fresh gas particle reaches the outlet port (short-circuiting).
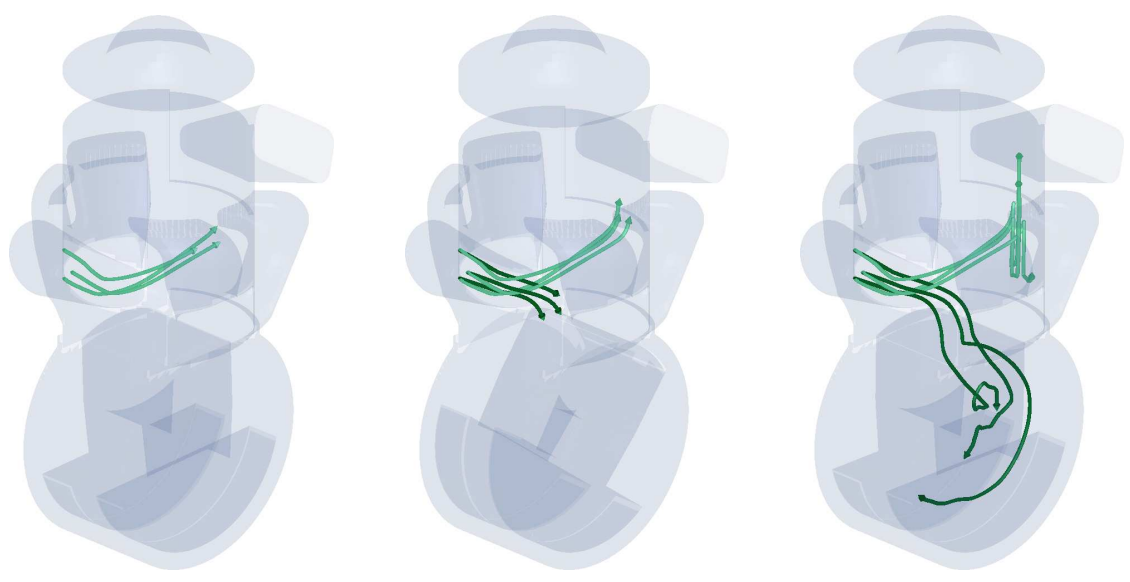

Figure 10.6.6: Visualization of the flow structure: Particle traces at $0^{\circ}$ (left), $40^{\circ}$ (middle), and $360^{\circ}$ crankshaft angle atdc (right). The dark green particles start at identical positions but $75^{\circ}$ crank angle later. Their path is completely distinct from the earlier ones.

A very good method to display the scavenging process in detail is the three-dimensional iso-level routine. All points with a given value are connected to form a surface. Figures 10.6.7 on the following page, 10.6.8, and 10.6.9 display four different fresh gas concentrations $(0.1,0.2,0.5$, and 0.8$)$ at three different times during the scavenging $\left(150^{\circ}, 180^{\circ}\right.$, and $220^{\circ}$ crank angle atdc). The scavenging loss by short-circuiting is clearly visible in Figure 10.6.7 (top left) and especially in the following Figure 10.6.8 (top and bottom left). Here, also the iso-surface structure 
can be compared to the Jante patterns (see Paragraph 10.6.6 on page 190). In Figure 10.6.8, a "tongue" pattern is evident. According to Jante, this leads not to an optimum scavenging, a high concentration of fresh gas reaches the exhaust port before it is closed.
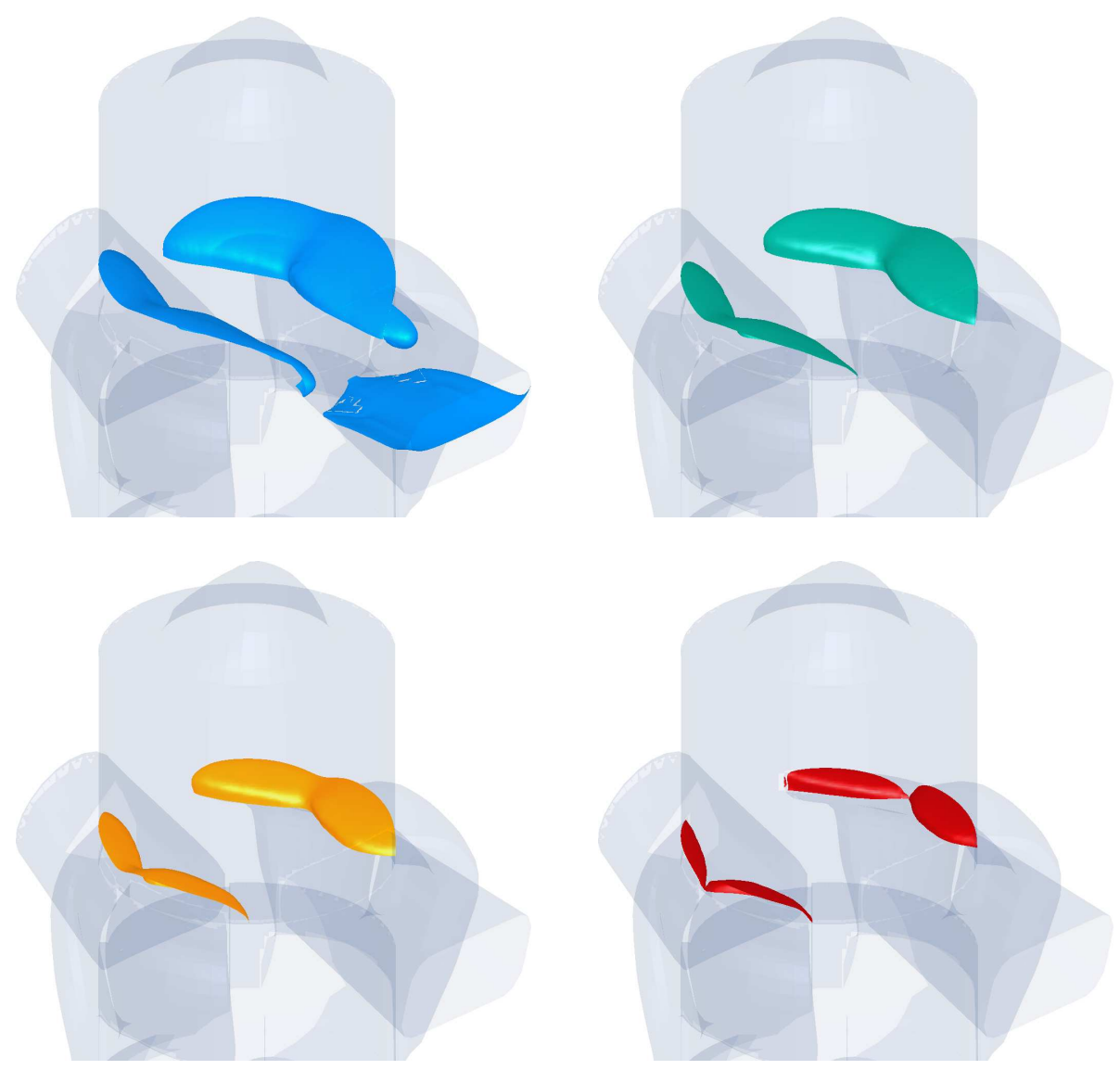

Figure 10.6.7: Visualization of the flow structure: iso-surfaces of fresh gas concentration at $150^{\circ}$ crank angle atdc for values of 0.1 (top left), 0.2 (top right), 0.5 (bottom left), and 0.8 (bottom right).

Objects, that move with the flow and are deformed by it, visualize the timedependent structure of this flow very intuitively (as shown in the series of pictures in Figure 10.6.10 on page 185). Here, the main problems of the geometry are demonstrated as well. A portion of the exhaust gas (transporting the blue ball) is left in the cylinder, and the short-circuiting transports a part of the red ball out of the exhaust port. The horseshoe-like shape of the blue exhaust object again reveals the "tongue" pattern of the scavenging flow.

With the isoline display, a more in detail view is possible. Figures 10.6 .11 and 

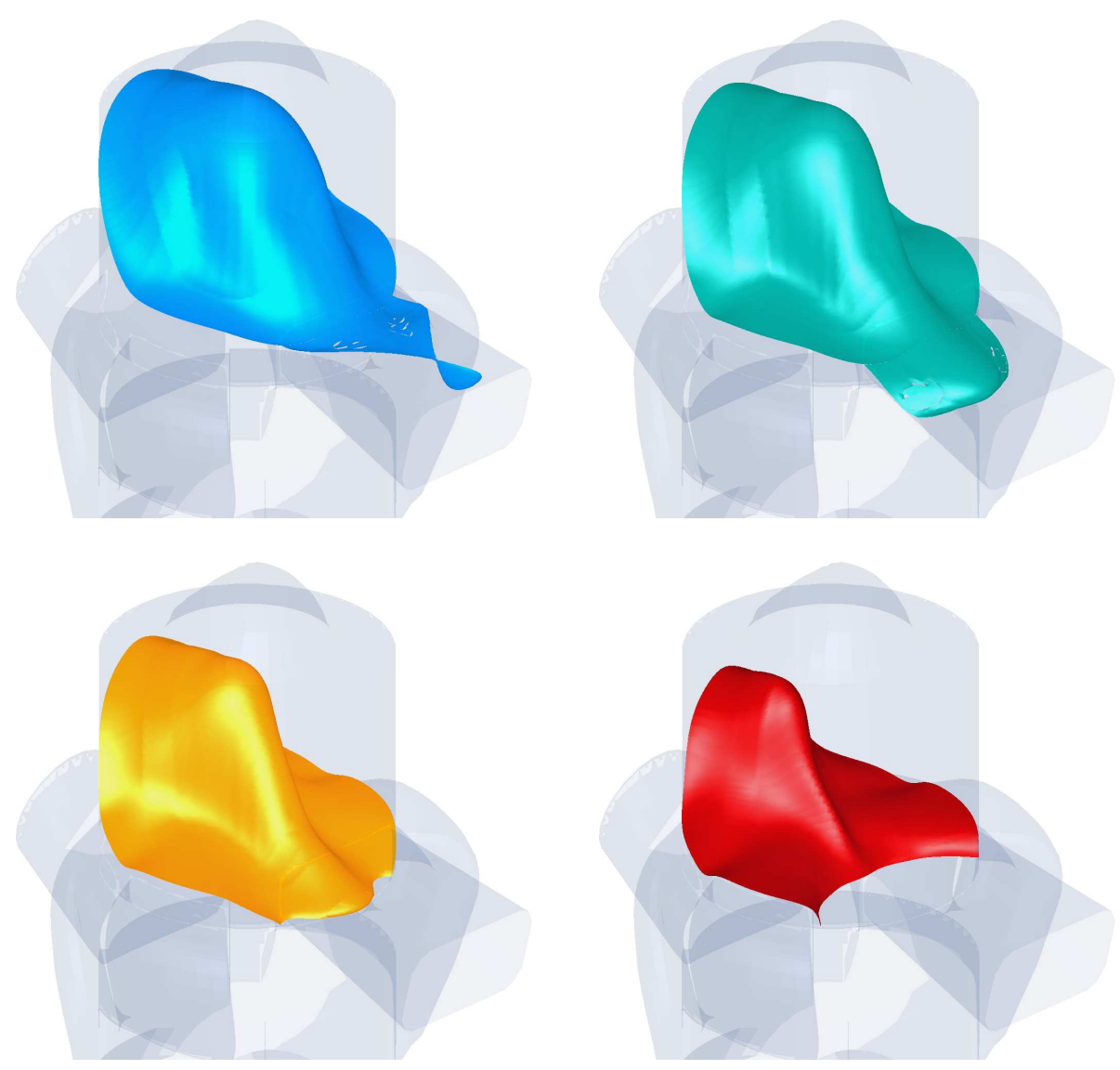

Figure 10.6.8: Visualization of the flow structure: iso-surfaces of fresh gas concentration at $180^{\circ}$ crank angle atdc for values of 0.1 (top left), 0.2 (top right), 0.5 (bottom left), and 0.8 (bottom right). 

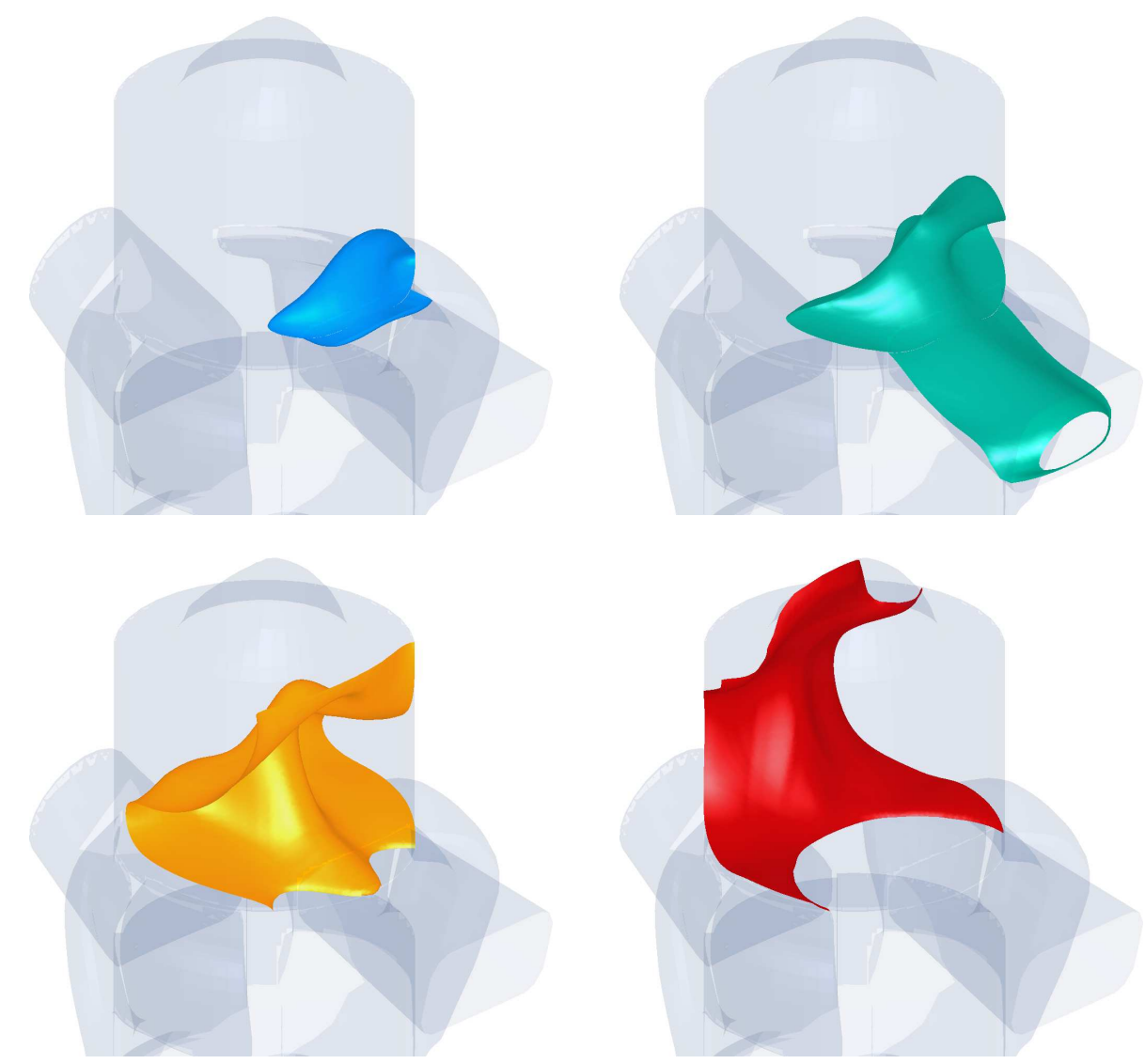

Figure 10.6.9: Visualization of the flow structure: iso-surfaces of fresh gas concentration at $220^{\circ}$ crank angle atdc for values of 0.1 (top left), 0.2 (top right), 0.5 (bottom left), and 0.8 (bottom right). 

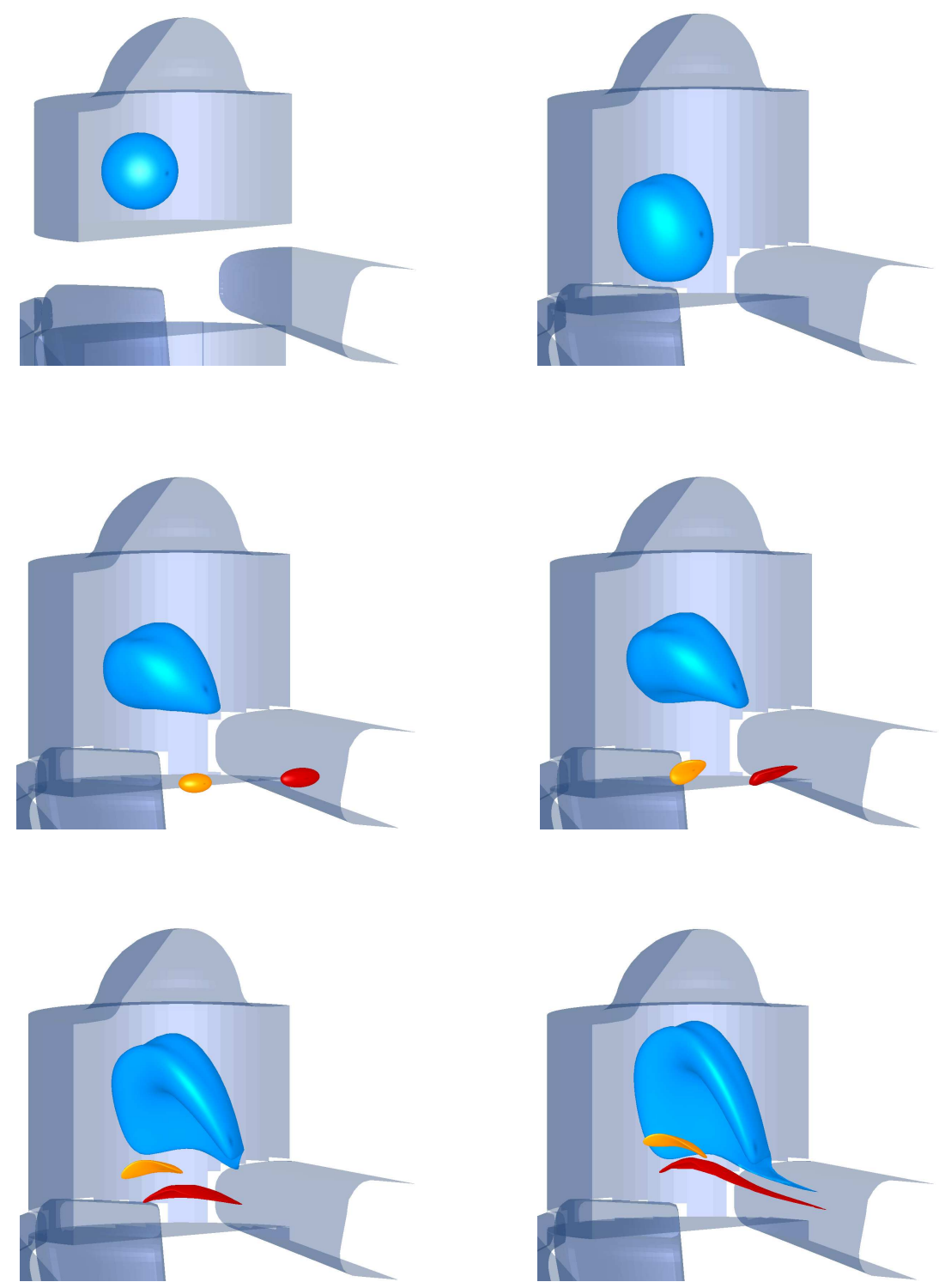

Figure 10.6.10: Visualization of the flow structure: Objects moving with the flow at $90^{\circ}$ (upper left), $140^{\circ}$ (upper right), $150^{\circ}$ (middle left), $180^{\circ}$ (middle right), $190^{\circ}$ (bottom left), and $210^{\circ}$ crankshaft angle atdc (bottom right). The blue ball moves with the exhaust gas flow, the red one starts in the main transfer port, and the orange one in the auxilliary transfer port. 
10.6.12 show the possibilities of this kind of visualization technique. Also, in these figures the "tongue" pattern of the flow at $180^{\circ}$ crank angle (middle part of the figures) is visible, resulting in a loop scavenging loss towards the end of the scavenging process (lower part of the figures). But this is not as damaging as the short-circuit flow from the main transfer port at the beginning of the scavenge process until bdc.

For the visualization of the flow velocities we refer to Paragraph 10.6.7 on page 190, where this method is used to analyze the boundary between the uprising fresh charge and the displaced exhaust gas.

\subsubsection{Characteristical Diagrams}

Apart from the visualization of the flow through the two-stroke engine, it is important to quantitatively analyze the quality of the geometry by the study of characteristical values. These can be plotted into diagrams to give a better impression of temporal development of the flow. It has been described in Paragraph 2.7.1 on page 23 what kind of diagrams are common for the analysis of the scavenging process. The most important include scavenge ratio (SR)-trapping efficiency (TE), scavenge ratio (SR)-scavenging efficiency (SE), and scavenge ratio (SR)-purity at exhaust port entry. Furthermore, the temporal development of the massflow of the fresh and exhaust gas at the transfer ports and the outlet port is very useful.

In the following diagrams in Figure 10.6.13 on page 189 the scavenge ratio value is measured during one rotation. It is therefore a temporal parameter and does not represent different engine loads. What can be seen in the purity at exhaust port entry graph in Figure 10.6.13 (upper left part) is a non-zero purity right from the beginning, i.e. a short-circuit flow reaches the outflow port very fast. This result can also be seen in the trapping efficiency diagram in the lower part of this figure. The trapping efficiency falls rapidly to a value of about .976 due to this short-circuiting. (For a detailed investigation to this short-circuiting see Section 10.11 on page 199.) As explained in Paragraph 2.3.2 on page 13, in our setup the scavenging efficiency (whose graph is in the upper right part of Figure 10.6.13) is identical to the purity of the fresh charge in the cylinder, due to no unburnt fresh charge after combustion. The final value of .673 is above the required level for a proper combustion process. Thus, no "four-stroking" takes place at this load. The vertical parts at the upper end of the scavenge ratio in the three diagrams are caused by the closing of the transfer ports prior to the outlet port.

Figure 10.6.14 on page 189 displays the two situations at the transfer portcylinder connection (left) and the outlet port entry (right). Here a more detailed view of the development of the massflow can be obtained. On the right diagram one can see the loss of fresh charge through the outlet port which has a peak at $194^{\circ}$ crank angle atdc (red curve). 

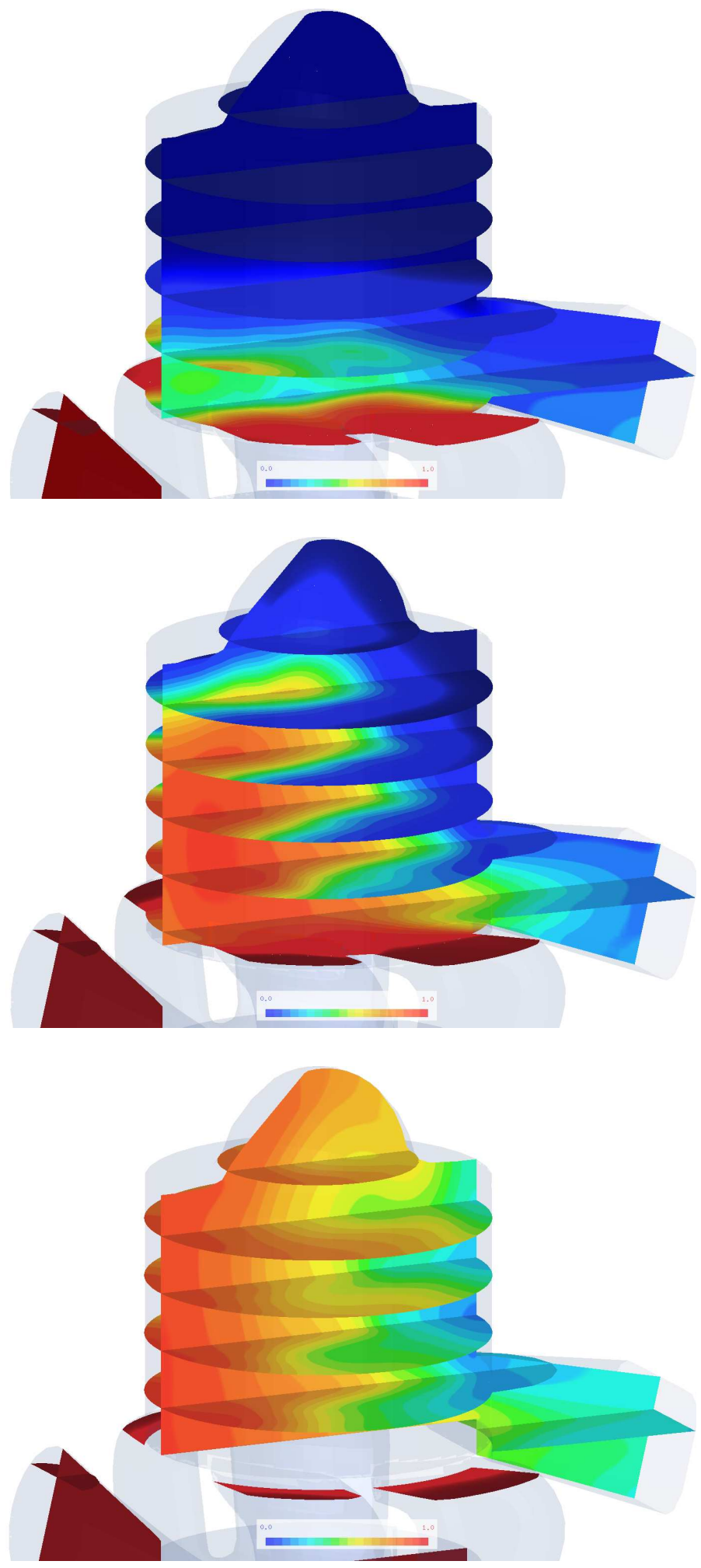

Figure 10.6.11: Visualization of the flow structure: horizontal clipping planes of iso-lines of the fresh gas concentration at $160^{\circ}$ (top), $180^{\circ}$ (middle), and $220^{\circ}$ (bottom) crank angle atdc. 

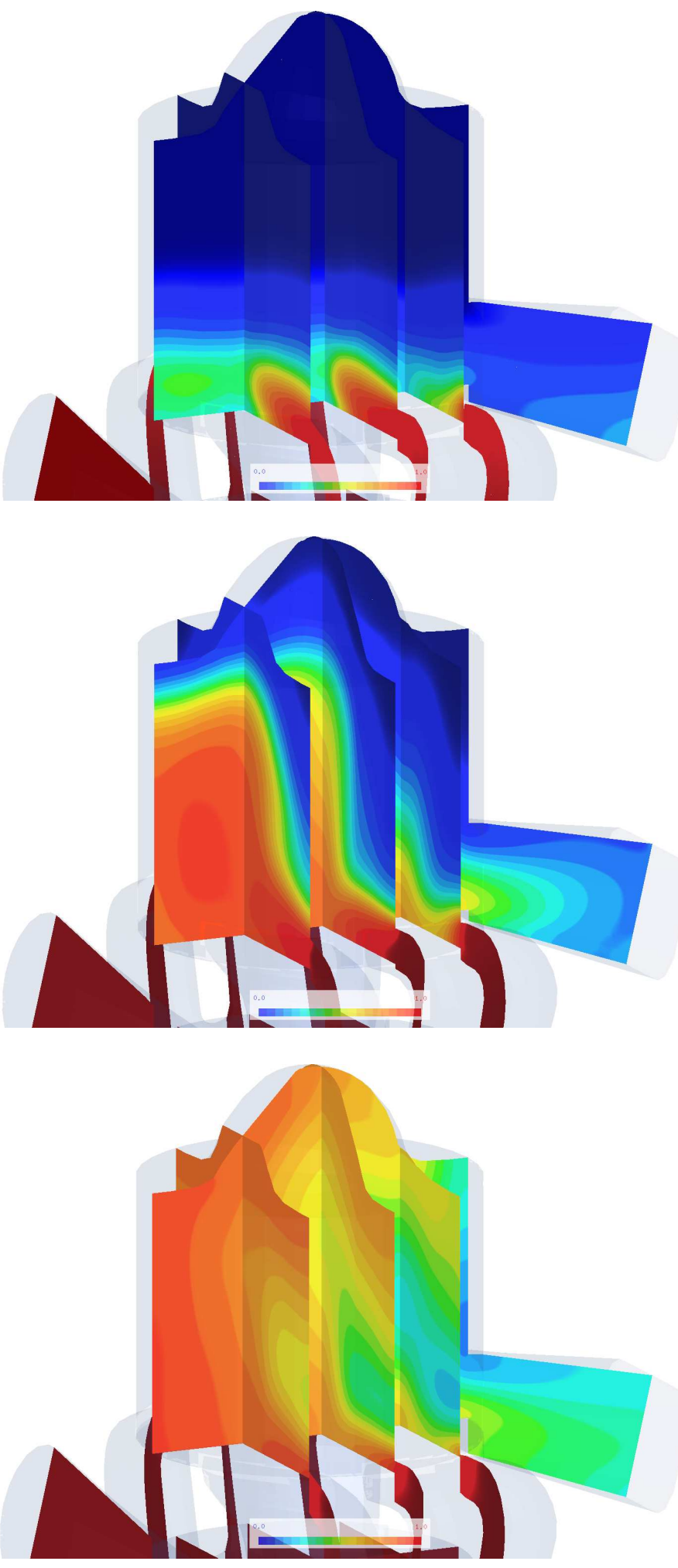

Figure 10.6.12: Visualization of the flow structure: vertical clipping planes of iso-lines of the fresh gas concentration at $160^{\circ}$ (top), $180^{\circ}$ (middle), and $220^{\circ}$ (bottom) crank angle atdc. 

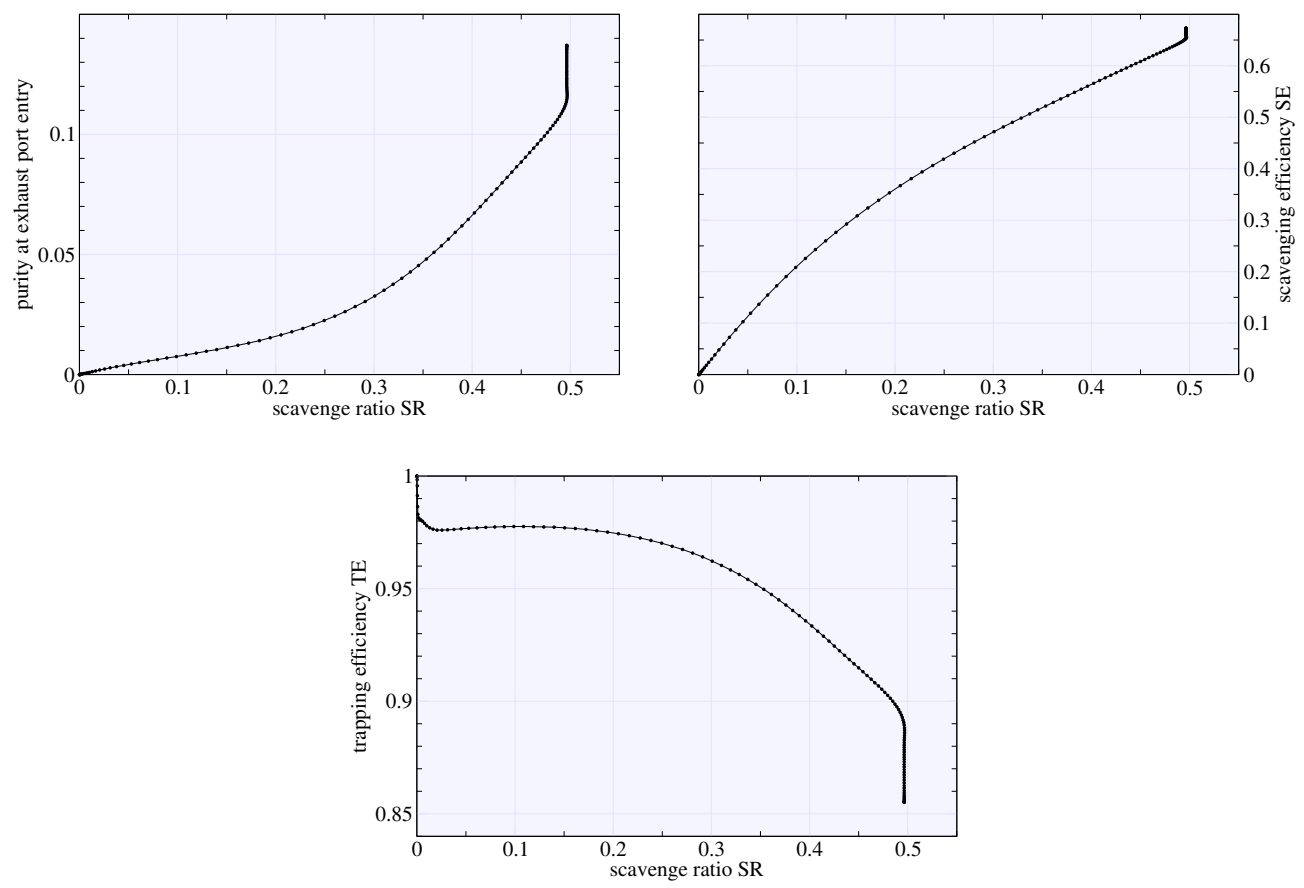

Figure 10.6.13: Characteristical diagrams: the scavenge ratio SR-purity at exhaust port entry graph (upper left), the scavenge ratio SR-scavenging efficiency SE (upper right), and the scavenge ratio SR-trapping efficiency TE graph (below).
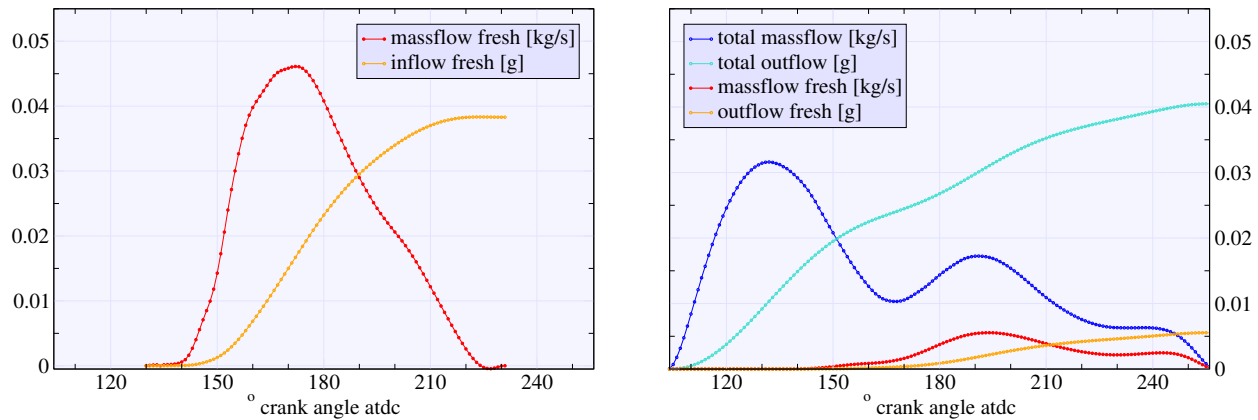

Figure 10.6.14: Characteristical diagrams: the massflow (red) and integrated massflow (orange) of fresh gas at the transfer port-cylinder connection (left), and the massflow (blue) and integrated massflow (turquoise) of the total charge and of the fresh charge (red/orange) at the outlet port entry (right). 


\subsubsection{Jante Test}

The Jante Test has been described in Paragraph 2.7.2 on page 24. The object of this test is to interpret the flow field at a horizontal cut through the cylinder half way between the piston position at bdc and the cylinder head. Some typical flow patterns have been described and evaluated. By comparison with these typical flow patterns the quality of the scavenging process of the two-stroke engine in question can be estimated. The fresh-gas distribution at this horizontal cut is shown in Figure 10.6.15. Compared to the typical patterns a "tongue" pattern can be observed in our engine geometry (cf. Figure 2.7.2 on page 24). This was already made visible by the iso-surfaces at bdc, as shown in Figure 10.6.8 on page 183.

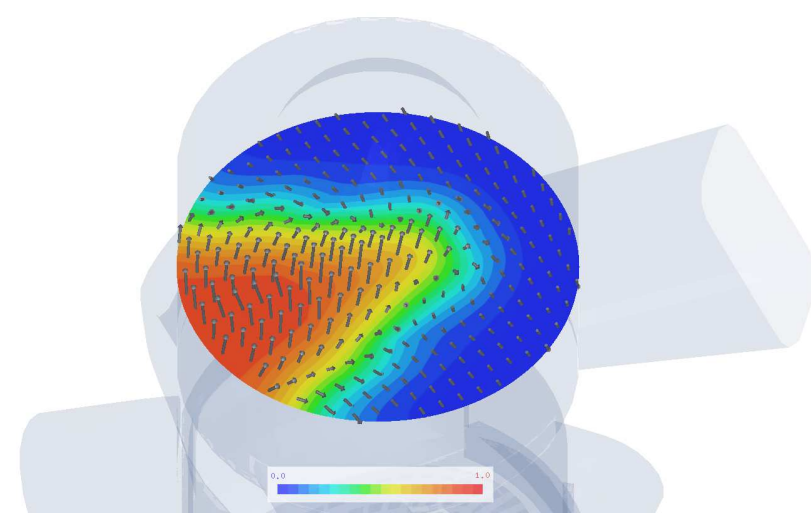

Figure 10.6.15: Jante test: depicted is the velocity field with the fresh charge concentration at the central horizontal clipping plane through the cylinder at bdc.

\subsubsection{Sharp Vertical Velocity Boundary}

As stated in Paragraph 2.7.3 on page 25, the sharp vertical velocity boundary between the "up" and "down" parts of the looping flow is characteristic for engines with good scavenging characteristics. Thus, in Figure 10.6.16 on the next page this velocity at bdc is presented. It can be observed that there is no particularly sharp transition in the vertical velocity between the fresh mixture and the exhaust gas. This brings up the assumption of a non-optimum scavenging process (as explained in [B196]).

\subsubsection{Deviation Angles}

A further interesting matter is the deviation of the flow from the intended direction. This topic has been introduced in Paragraph 2.7.4 on page 25. Figure 10.6.17 on the next page gives the results for our geometry at bdc. Especially for the horizontal direction a considerable deviation is observed. This can also be seen in Figure 10.6.18 on page 192, where the time-dependent deviation is plotted. 


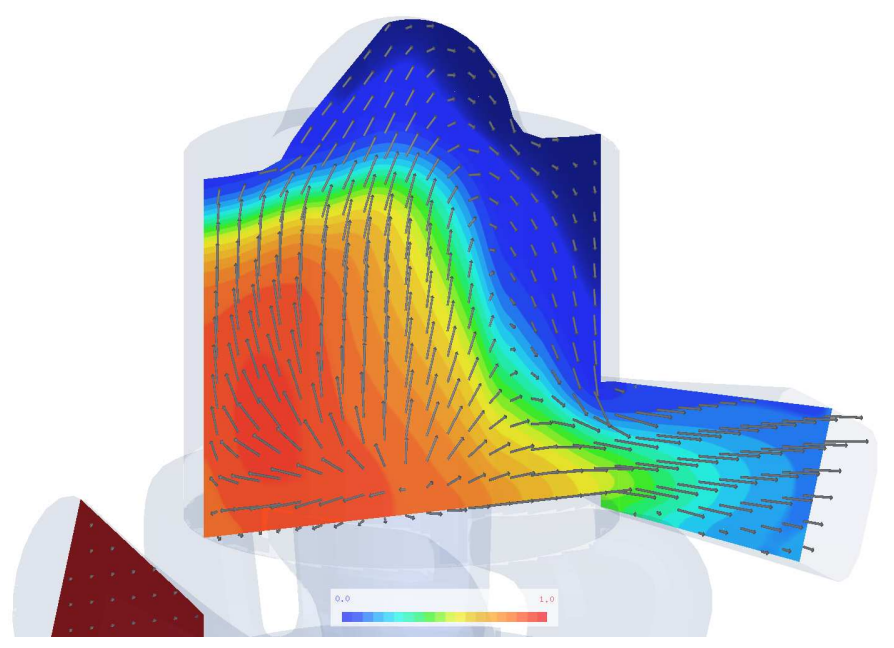

Figure 10.6.16: Sharp vertical velocity boundary: depicted is the velocity field with the fresh charge concentration at the central longitudinal clipping plane at bdc.

Thus, the time has come to support the empirical recommendations for port plan layout by three-dimensional CFD calculations (cf. [B196]).
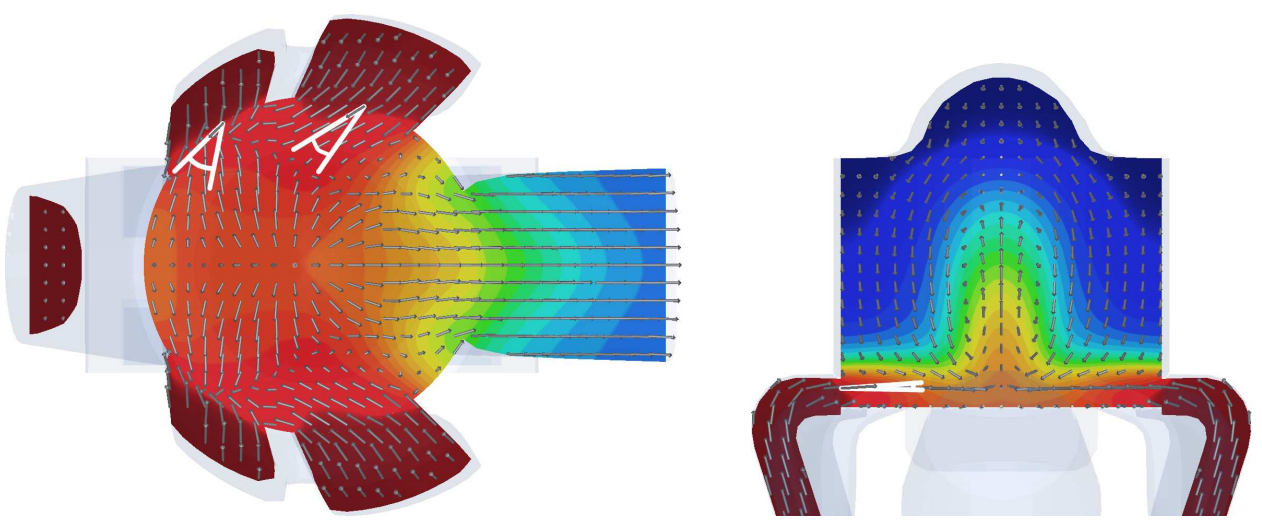

Figure 10.6.17: Deviation angles: horizontal (left) and vertical (right) deviation of the flow from the intended direction at bdc (the velocity as well as the fresh charge concentration are shown).

\subsubsection{Squish velocity}

The squish velocity is not directly linked to the scavenging process (see also Paragraph 2.7.5 on page 26). But as the velocity data in the cylinder is available it is easy to compute it. The squish velocity is an important indicator for the quality of the combustion process. A good squishing action mixes the gas intensively and the combustion takes place without detonations. The plot of the squish velocity relative 

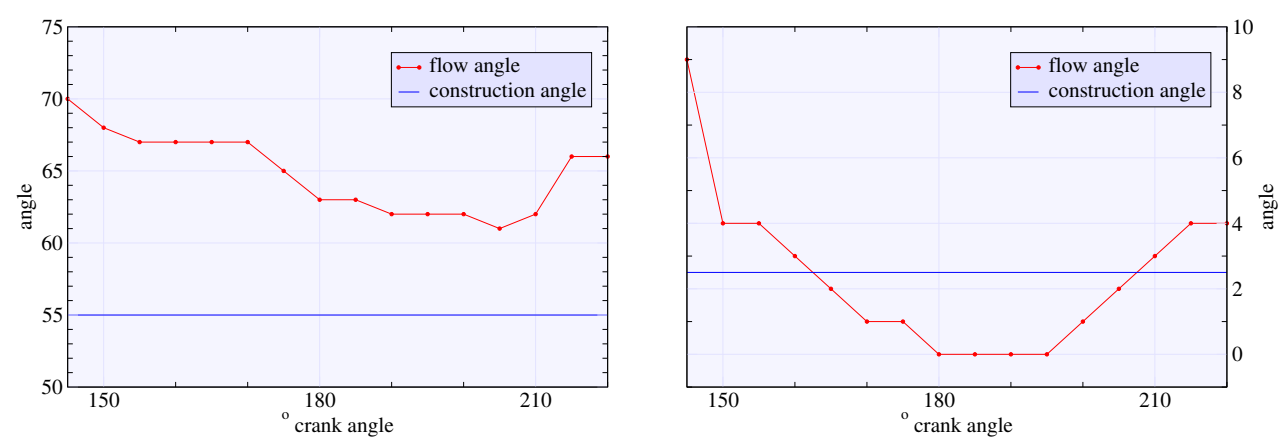

Figure 10.6.18: Deviation angles: comparison of the geometrical design direction (blue curve) and the real flow angle (red curve) in the horizontal (left) and vertical (right) plane.

to the piston position is shown in Figure 10.6.19. Figure 10.6.20 on the next page gives a more detailed view of the velocity in the cylinder at $20^{\circ}$ crank angle btdc. A pronounced squishing action can be observed, which facilitates the mixture of the fresh charge and the remaining exhaust gas.

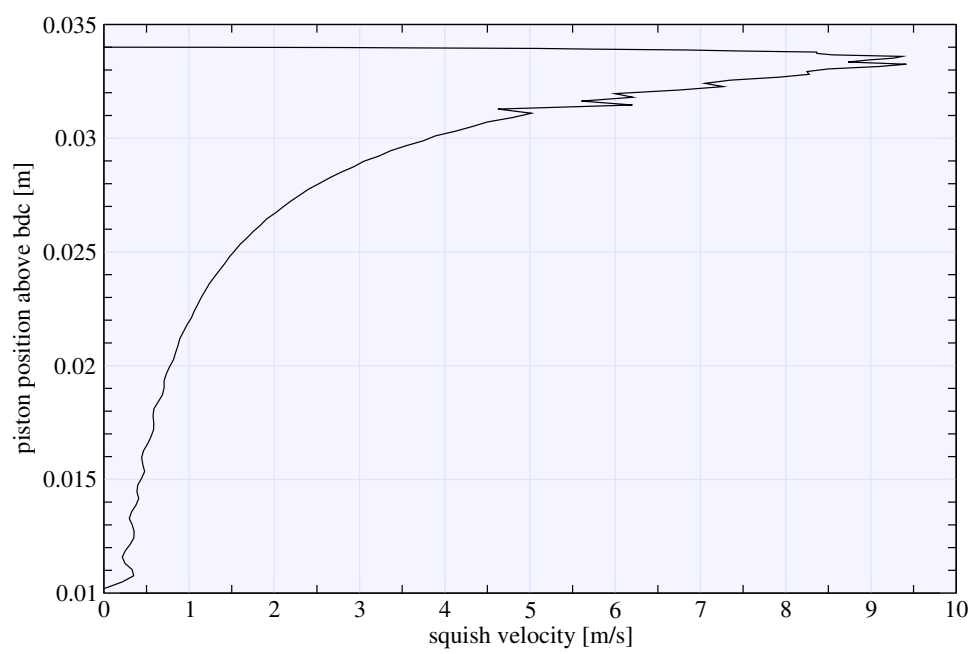

Figure 10.6.19: Squish velocity: radial velocity in the cylinder at a vertical distance of 16 $\mathrm{mm}$ from the center axis $x=z=0$.

\subsection{Efficiency of the Adaptive Strategies}

\subsubsection{Efficiency of the Adaptive Local Time-Stepping}

The efficiency of the temporally consistent adaptive local time-stepping has been tested at the real engine simulation, because its highest benefit is obtained with geometries consisting of different size elements, which is usually the case in applied 


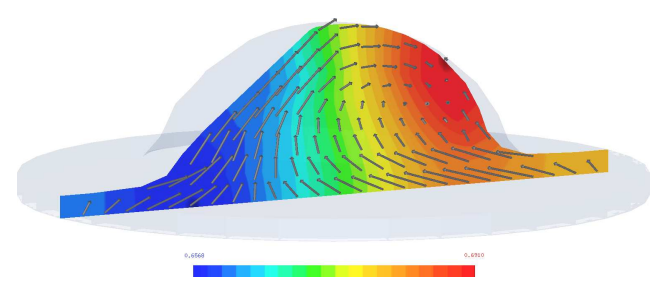

Figure 10.6.20: Squish velocity: velocity vectors and fresh gas concentration on a central vertical clipping plane through the cylinder at $20^{\circ}$ btdc.

real-world problems. Six calculations have been conducted to study the efficiency of the local time-stepping. The simulations are governed by the data stated in the above Sections 10.1 on page 167, 10.2 on page 170, and 10.3 on page 172 . The meshes are a globally once refined 60000 element mesh, resulting in approximately 500000 elements, a twice globally refined 15000 element mesh, with 1000000 elements, and a twice globally refined 30000 element mesh, with 2000000 elements. On each of these meshes a simulation with local time-stepping and one with global time-stepping was conducted. The parameters for the local time-stepping (see Section 6.1 on page 87) setup are:

$$
l_{\text {max }}:=4 \quad l_{\text {geom }}:=2
$$

The distribution of the elements in the individual $\Delta t$-levels at $10^{\circ}$ crank angle atdc is shown in Figure 10.7.1 and the spacial distribution in the mesh in Figure 10.4.1 on page 174 .

The results of this investigation can be seen in Figure 10.7.2 on the following page (the time need is extrapolated from a calculation for the first $20^{\circ}$ crank angle). On each consecutive mesh the time need more than doubles. This is due to the doubling of the number of elements and the decreasing step-size $\Delta t$. What is remarkable is the fact that the simulation with local time-stepping on the 1000000 and 2000000 element mesh is faster than the one with global time-stepping on a mesh with half as many elements. The resulting speed-up factor is presented in Figure 10.7.2 (right).

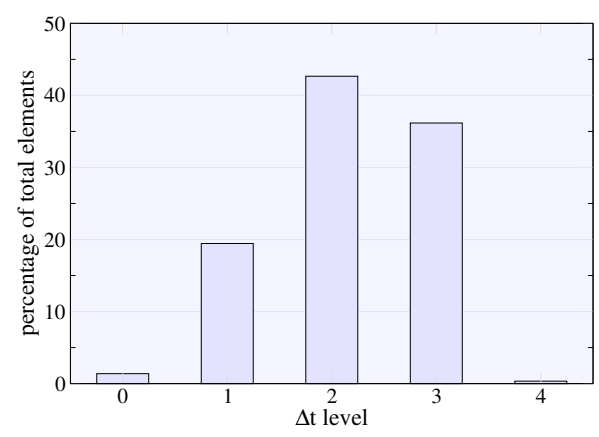

Figure 10.7.1: Efficiency of the local time-stepping: the distribution of the elements into the $\Delta t$-levels at $10^{\circ}$ crank angle atdc. 

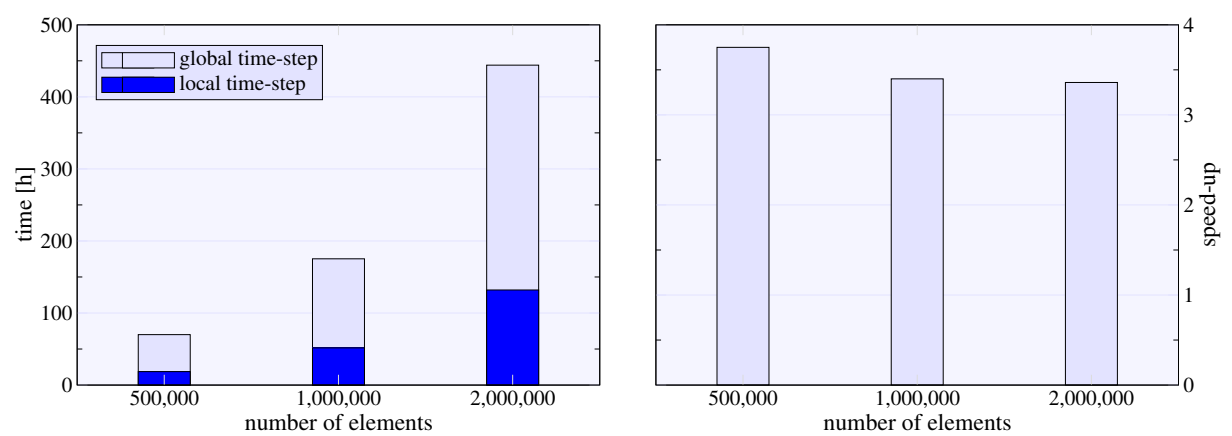

Figure 10.7.2: Efficiency of the local time-stepping: needed CPU-time (AMD Opteron 64 bit processor) for the calculations on the different meshes with and without local time-stepping for one revolution of the crankshaft (left) and the resulting speed-up factor (right).
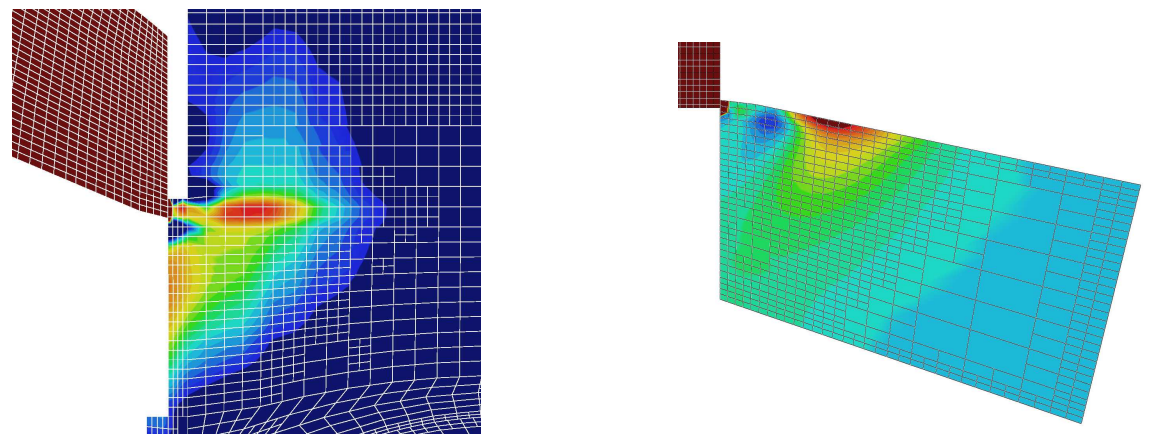

Figure 10.7.3: Efficiency of the local mesh adaption: adaptive refinement of the mesh at the opening of the inlet at $290^{\circ}$ (clipping plane of density and mesh on the left-hand side) and the opening of the outlet at $110^{\circ}$ crank angle atdc (clipping plane of pressure and mesh on the right-hand side).

\subsubsection{Efficiency of the Dynamic Local Mesh Adaption}

The efficiency of this method can be estimated by comparing the number of elements of an adaptively refined mesh with the number on a globally refined one (compare test case 9.4.2 on page 148). The parameters for the marking strategy (as described in Section 6.2.4 on page 94) are given as follows:

$$
C_{\text {refine }}:=1.1 \quad C_{\text {coarsen }}:=0.6
$$

The mesh is checked for refinement every $4^{\text {th }}$ time step and for coarsening every $32^{\text {nd }}$ time step. All elements on the walls of the geometry and at the connection of the different mesh parts are also refined.

In Figure 10.7.3 the refinement of the elements can be seen, as the steep gradients of the two pulses enter the crankcase and the exhaust port respectively. Also, the wall and connection refinements are visible.

Figure 10.7.4 on the facing page gives the results of the calculations on a twice refined 15000 element macro mesh (left), on a twice refined 30000 element macro 
mesh (middle), and a twice refined 60000 element macro mesh (right). The number of elements of the adaptively refined mesh (dark blue) is in the 15000 element mesh on average $16 \%$ lower than in the globally refined mesh (light blue), in the 30000 element mesh $21 \%$ lower, and, in the 60000 element mesh $27 \%$ lower. Although the parameters for the marking strategy are adjusted for not too fine resolution, the dynamic in the flow forces the refinement of a relatively high percentage of elements. A higher efficiency of the local mesh adaption can be expected at higher levels of refinement and with finer meshes.
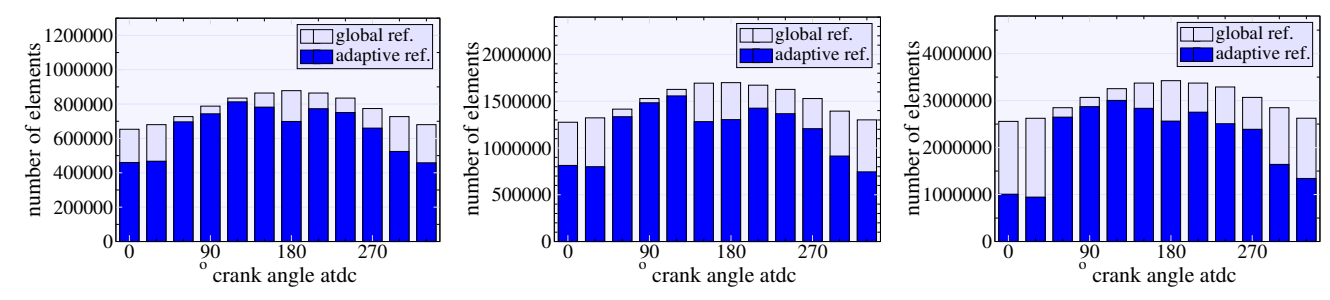

Figure 10.7.4: Efficiency of the local mesh adaption: shown is the number of elements of the adaptively refined mesh (dark blue) and the globally refined mesh (light blue) in the twice refined 15000 element macro mesh (left), in the twice refined 30000 element macro mesh (middle), and in the twice refined 60000 element macro mesh (right).

\subsection{The Execution Time Call-Graph}

For the profiling of the computation code the program KCacheGrind was used. The execution times of the routines for a typical configuration (cf. Section 10.2 on page 170) are presented in Figure 10.8.1 on the next page. Displayed are only routines needing more than $1.0 \%$ of the total execution time.

\subsection{Question One: The Effect of the Crankshaft Rota- tion}

The crankshaft rotation has a profound effect on the flow field in the crankcase itself (compare Figure 10.6.6 on page 181 with Figure 10.9.1 on page 197). But the interesting part is the flow field in the cylinder at the scavenging process. Is the rotating crankshaft changing the characteristic values which determine the quality of the engine, namely scavenge ratio (SR) and trapping efficiency (TE) as defined in Section 2.3.2 on page 13? Thus, does the engineer have to worry about an altered scavenging process due to design changes made in the crankcase?

\subsubsection{Setup of the Comparison}

We examine this question with our real engine geometry. For this, the engine is simulated with the data as given in the Sections 10.1 on page 167, 10.2 on page 170, 


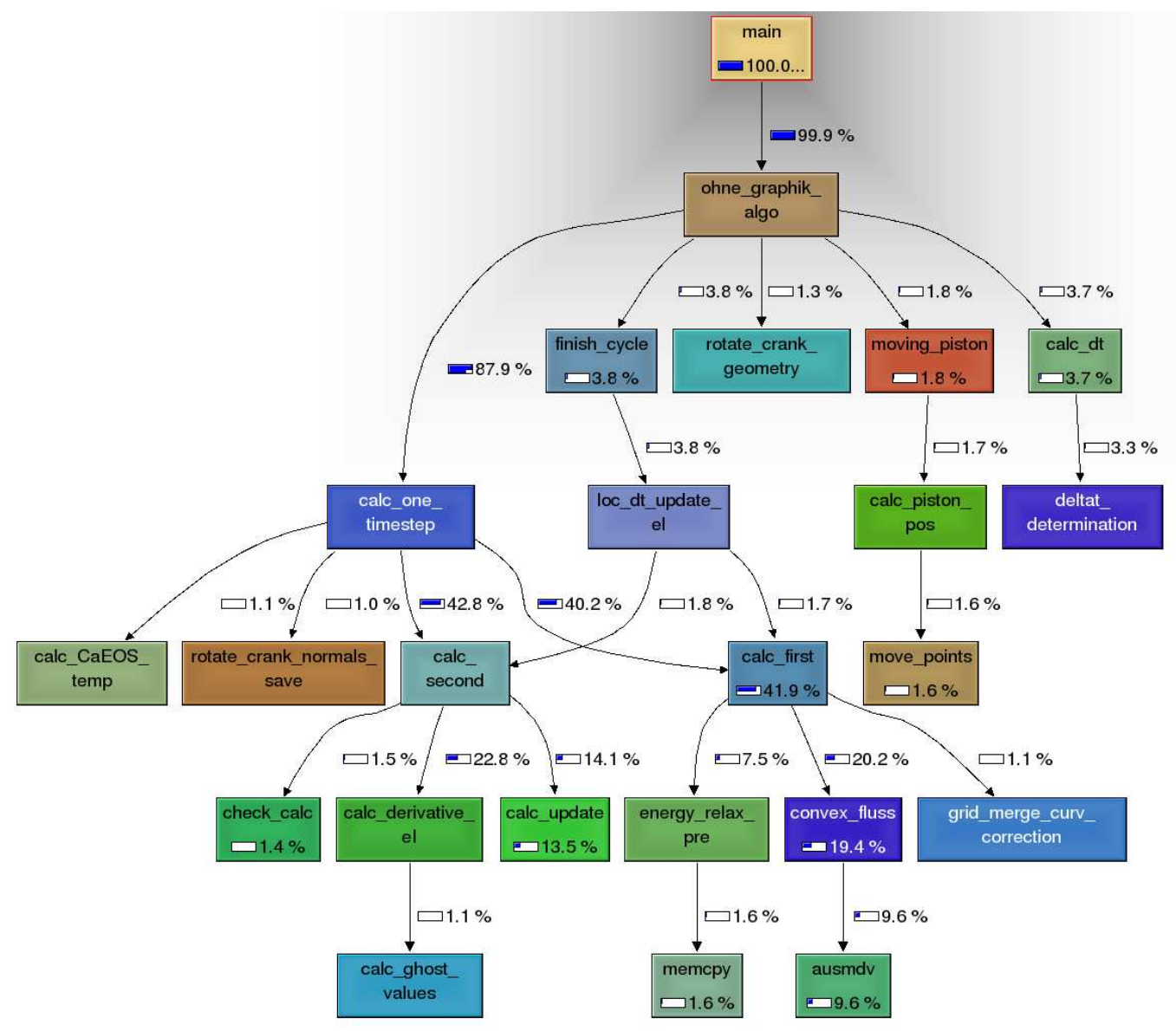

Figure 10.8.1: Call graph with execution time information. 
and 10.3 on page 172 . However, the crankshaft of the engine is fixed, and not rotating, in this second simulation. The piston motion is not affected by this procedure.

\subsubsection{Results}

In Figure 10.9.2 on the following page it is made clear that there is hardly any difference between the scavenging process with the rotating crankshaft and the one with fixed crankshaft. Thus, the big difference in the flow field in the crankcase has almost no influence on the scavenging process in the cylinder. The only driving force for the scavenging seems to be the pressure difference between crankcase and cylinder which is not altered by the crankshaft rotation. This might possibly change for configurations for which the port window of the transfer ports is partly shadowed by the crankshaft at certain angles. This obstruction for the flow from crankcase to cylinder can have a measurable influence on the scavenging process. But in our geometry this is not the case (see Figure 10.9.3 on the next page).
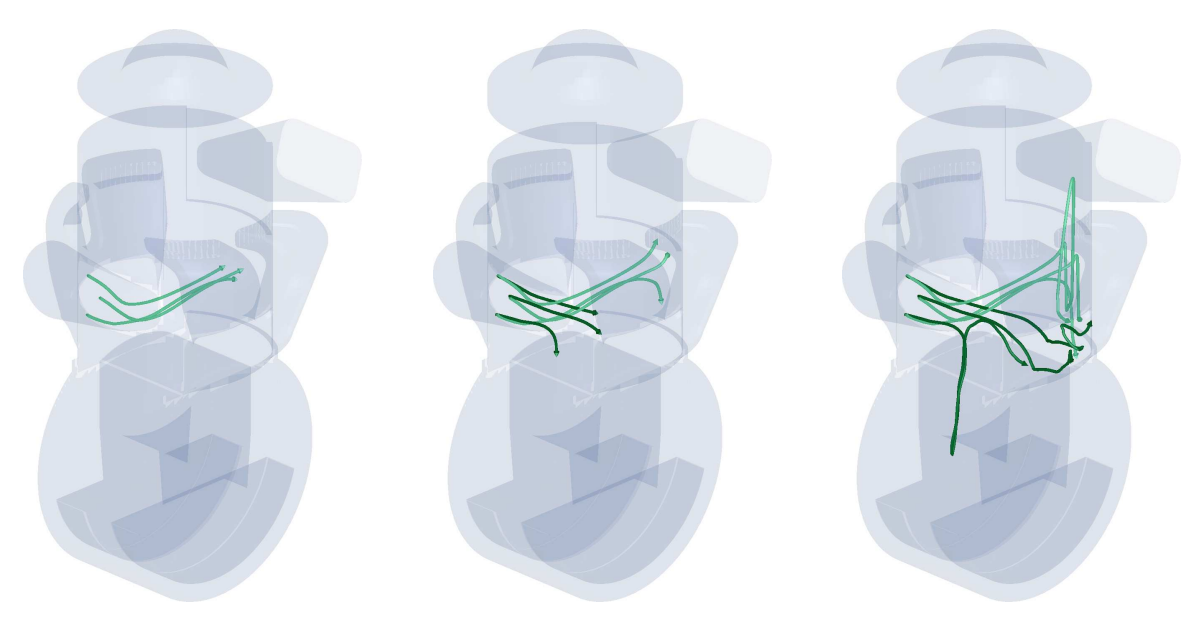

Figure 10.9.1: Effect of crankshaft rotation: Particle traces at $0^{\circ}$ (left), $40^{\circ}$ (middle), and $360^{\circ}$ crankshaft angle atdc (right) with non-rotating crankshaft. The dark green particles start at identical positions but $75^{\circ}$ crank angle later. Their path is particularly strongly influenced by the fixed crankshaft.

\subsection{Question Two: The Unsymmetrical Transfer Port}

One idea to obtain a better scavenging behavior of a two-stroke engine, is to force a slightly unsymmetrical flow field.

A further aspect of this test case is to check the robustness of the flow field with respect to small variations in the symmetry of the transfer ports, caused by tolerances in the production process. 


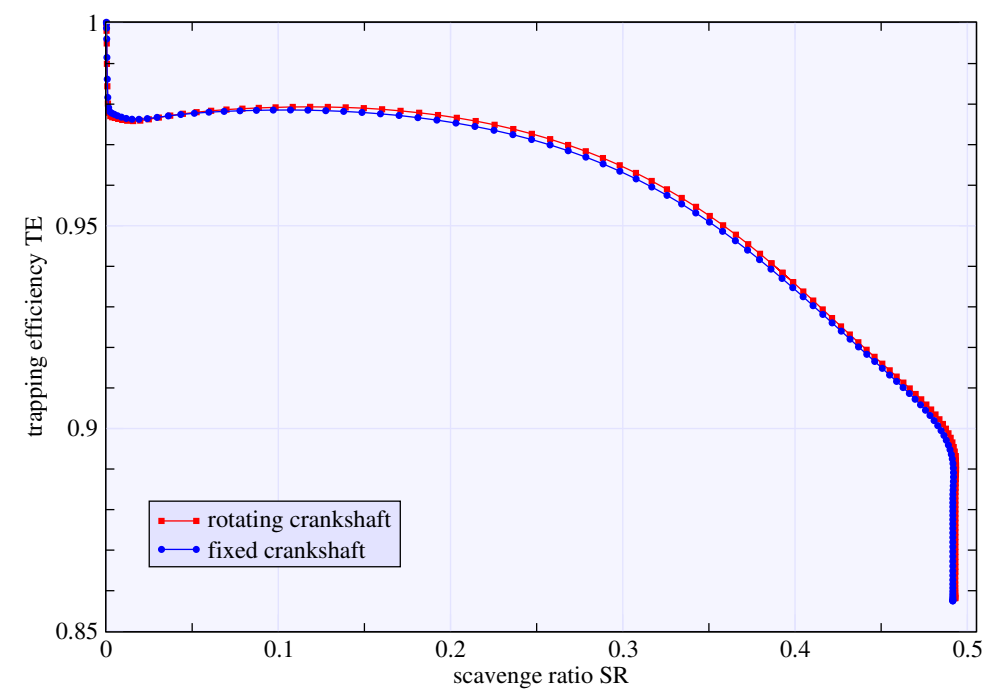

Figure 10.9.2: The effect of the crankshaft rotation: difference in the scavenge ratio-trapping efficiency graph.
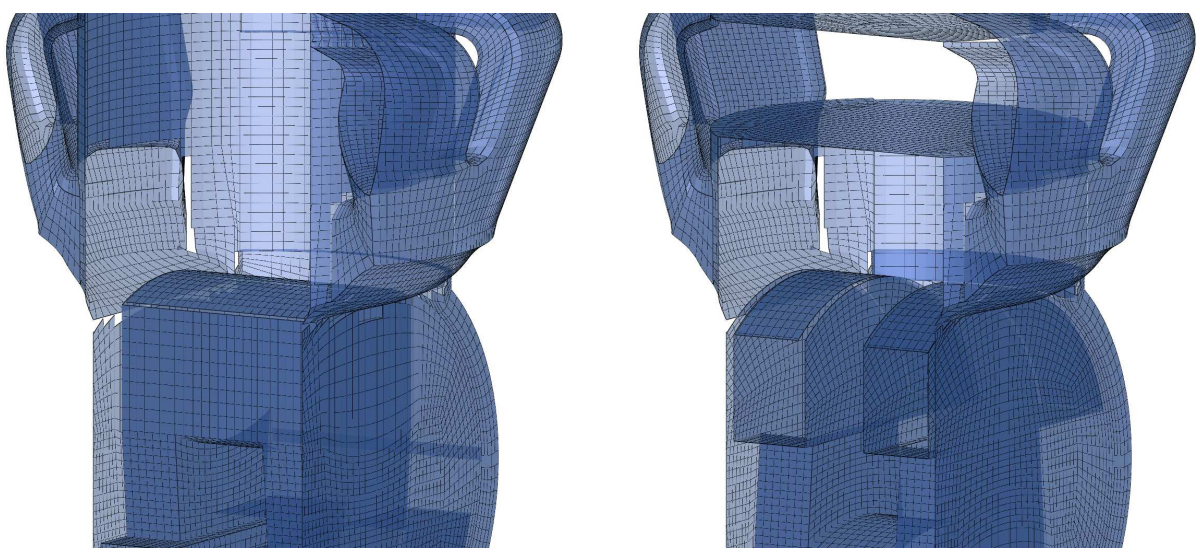

Figure 10.9.3: The effect of the crankshaft rotation: crankshaft at tdc (left) at bdc (right), there is almost no shadowing of the transfer port windows by the crankshaft. 


\subsubsection{Setup of the Comparison}

Two sets of transfer port meshes were generated. A symmetrical one and an unsymmetrical one (see Figure 10.10.1). The connection of the unsymmetrical transfer port to the crankcase is lifted for $2 \mathrm{~mm}$ on one side. Apart from this difference in the meshes, all parameters, as described in Sections 10.1, 10.2, and 10.3, remain unchanged in these two simulations.
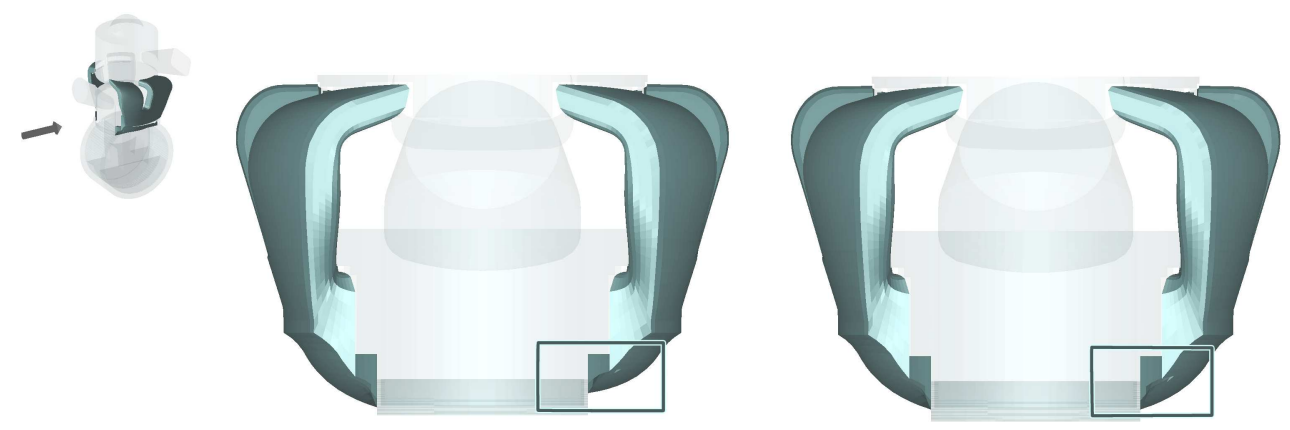

Figure 10.10.1: The unsymmetrical transfer port: unsymmetrical (left) and symmetrical (right) lower transfer port.

\subsubsection{Results}

As in the comparison between rotating and fixed crankshaft, almost no difference is visible in the trapping efficiency diagram in Figure 10.10.2 on the next page. Additionally, in the graphs in Figure 10.11 .1 on page 201 no unsymmetrical pattern can be detected. Therefore, in order to alter the flow structure, and to enhance the trapping efficiency by an unsymmetrical geometry, stronger variations, or variations in the upper part of the transfer ports are necessary.

On the other hand, the flow field is stable with respect to small variations in the lower part of the transfer ports. Thus, a realistic flow simulation can be assumed in this respect. No change in the flow structure is introduced by this asymmetric configuration.

\subsection{Question Three: The Study of Short-Circuiting}

The problem of short-circuiting is the main reason for the bad exhaust characteristic in a two-stroke engine. Unburnt hydrocarbons escape the combustion process and reach the exhaust, polluting the environment. Thus, the main task of the engineer is to minimize this short-circuiting. Therefore, it is advantageous to know which transfer port causes how much loss of fresh gas. 


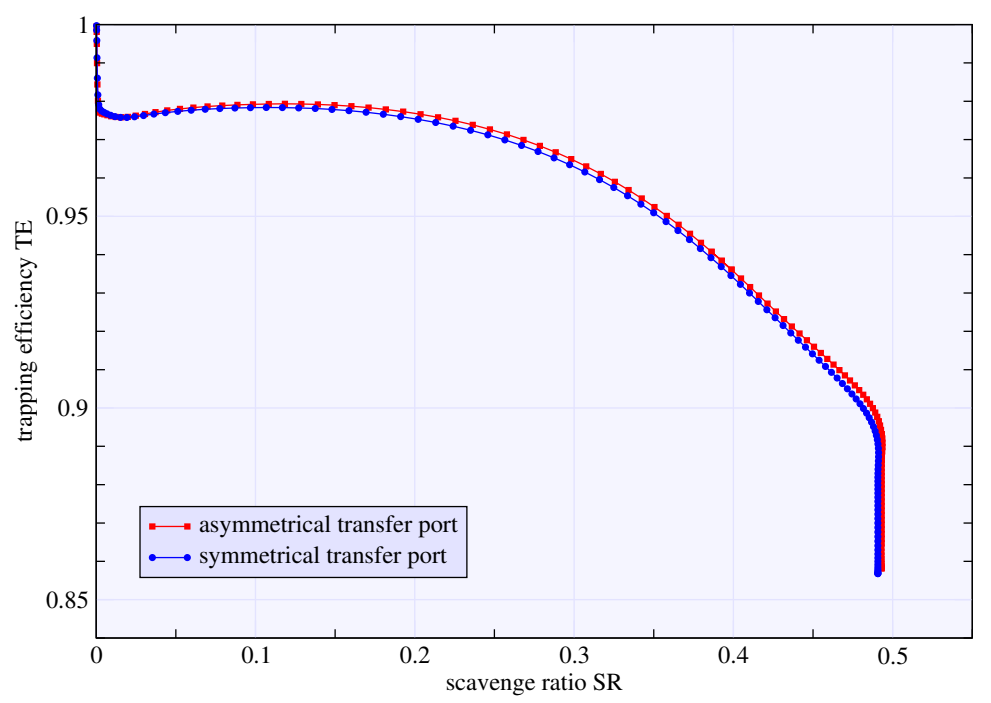

Figure 10.10.2: The unsymmetrical transfer port: difference in the scavenge ratio-trapping efficiency graph.

\subsubsection{Enhanced Fresh-Gas Tracking}

In order to derive the origin of the escaped fresh gas, four different fresh gas species $\sigma_{i}, 1 \leq i \leq 4$, that do not interact with each other, are introduced. This extension of the numerical scheme as described in Chapter 4 on page 45 , is straightforward. In the crankcase, the fresh charge is composed homogeneously of these four fresh gas species $\left(\sigma_{i}:=\frac{1}{4} \sigma, 1 \leq i \leq 4\right)$. But as soon as a transfer port is reached, all the fresh gas is shifted to one species depending on the transfer port: $\sigma_{j}:=\sum_{i=1}^{4} \sigma_{i}$ where $j$ depends on the port number. Now, if the fresh gas enters the cylinder it can be exactly traced back to its port of entry (see Figures 10.11.2 on page 202 and 10.11.3). If some fresh gas reaches the outlet duct, it can be quantitatively attributed to the single transfer ports (as shown in Figure 10.11.1 on the next page).

This fresh-gas tracking is applied to the engine simulation with the data as given in the Sections 10.1 on page 167, 10.2 on page 170 , and 10.3 on page 172 .

\subsubsection{Results}

In Figures 10.11.2 and 10.11.3 it can be guessed that the main transfer ports are the principal source of short-circuit losses. Design recommendations to improve the scavenging process might be derived from these figures.

In the diagrams shown in Figure 10.11.1 a further detailed analysis of the scavenging losses is possible. These losses can be classified into three distinct mechanisms (cf. [RKPM01]). The first one is the short-circuit loss. This occurs at the beginning of the scavenging. In the right graph of Figure 10.11.1 the main transfer ports (red/orange curve) account for this loss up to $165^{\circ}$ crank angle atdc (this can also be seen very nicely in the upper left image of Figure 10.6.7 on page 182). The second mechanism is the central loss between $165^{\circ}$ and $225^{\circ}$ crank angle atdc. Here the main transfer port also causes this kind of scavenging loss (already visible in the 
upper right part of Figure 10.6.8 on page 183). And finally, from $225^{\circ}$ crank angle atdc until the closing of the exhaust port, the loop scavenge loss, produced by all transfer ports, is the main cause for fresh charge loss at the end of the scavenging process (barely visible in the bottom left image of Figure 10.6 .9 on page 184).

What also can be seen, are the completely symmetrical losses of fresh charge. As these diagrams are based of the data from the simulation with unsymmetrical lower transfer ports, this is a further indication that the flow is nevertheless completely symmetric.
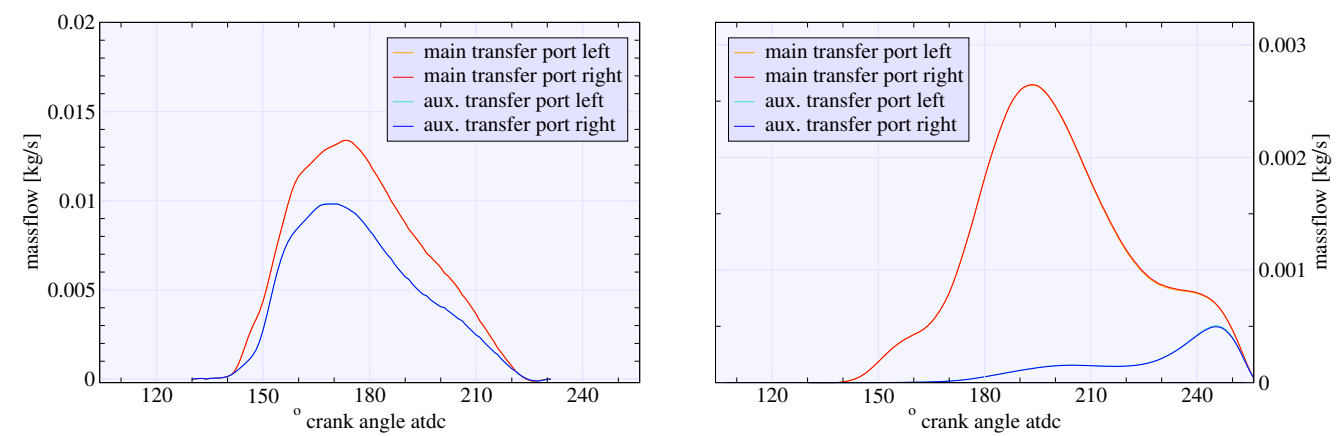

Figure 10.11.1: Fresh-gas tracking: the fresh charge massflow at the transfer port-cylinder connection (left) and at the exhaust port entry (right) attributed to the single transfer ports. 

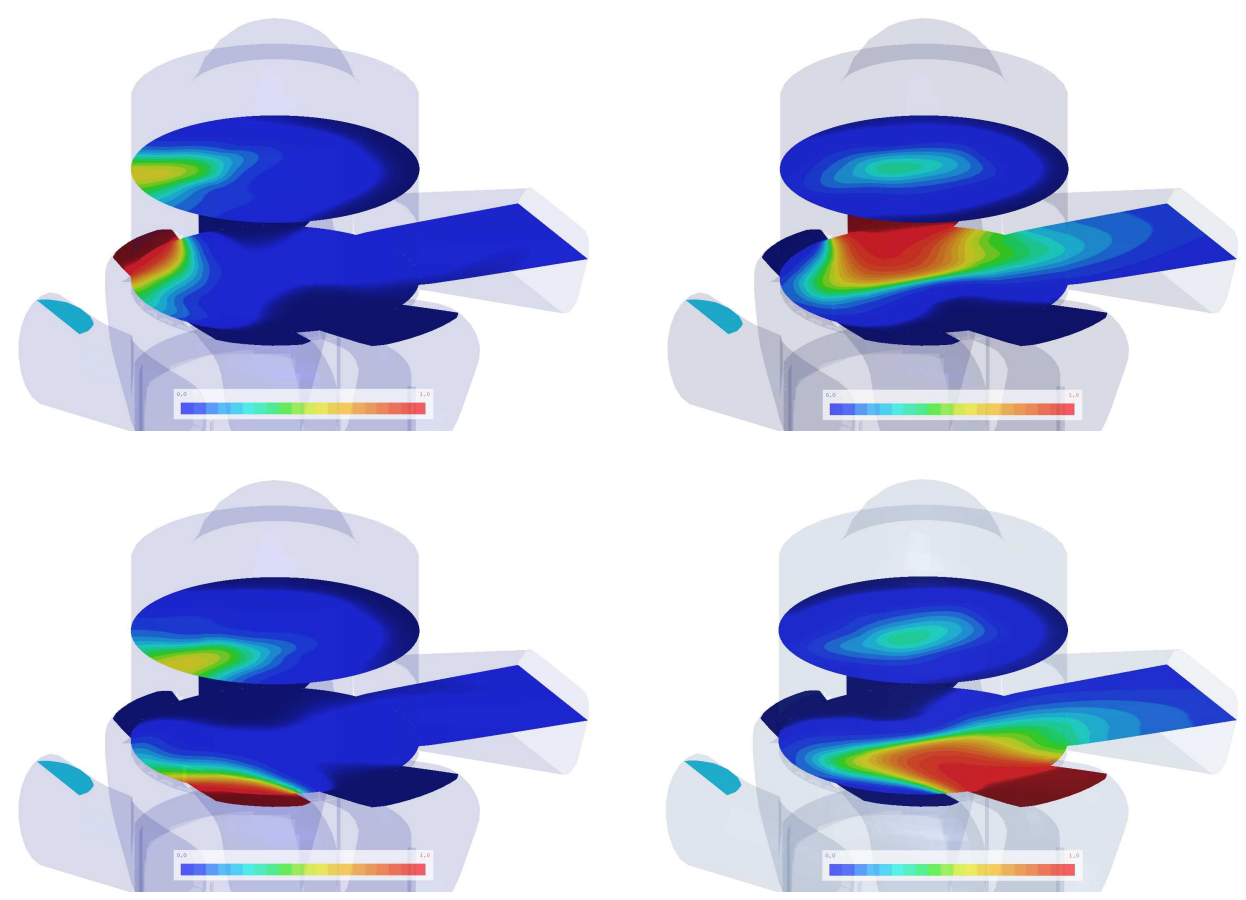

Figure 10.11.2: Fresh-gas tracking: shown are the fresh-gas concentration contours coming from each transfer port.
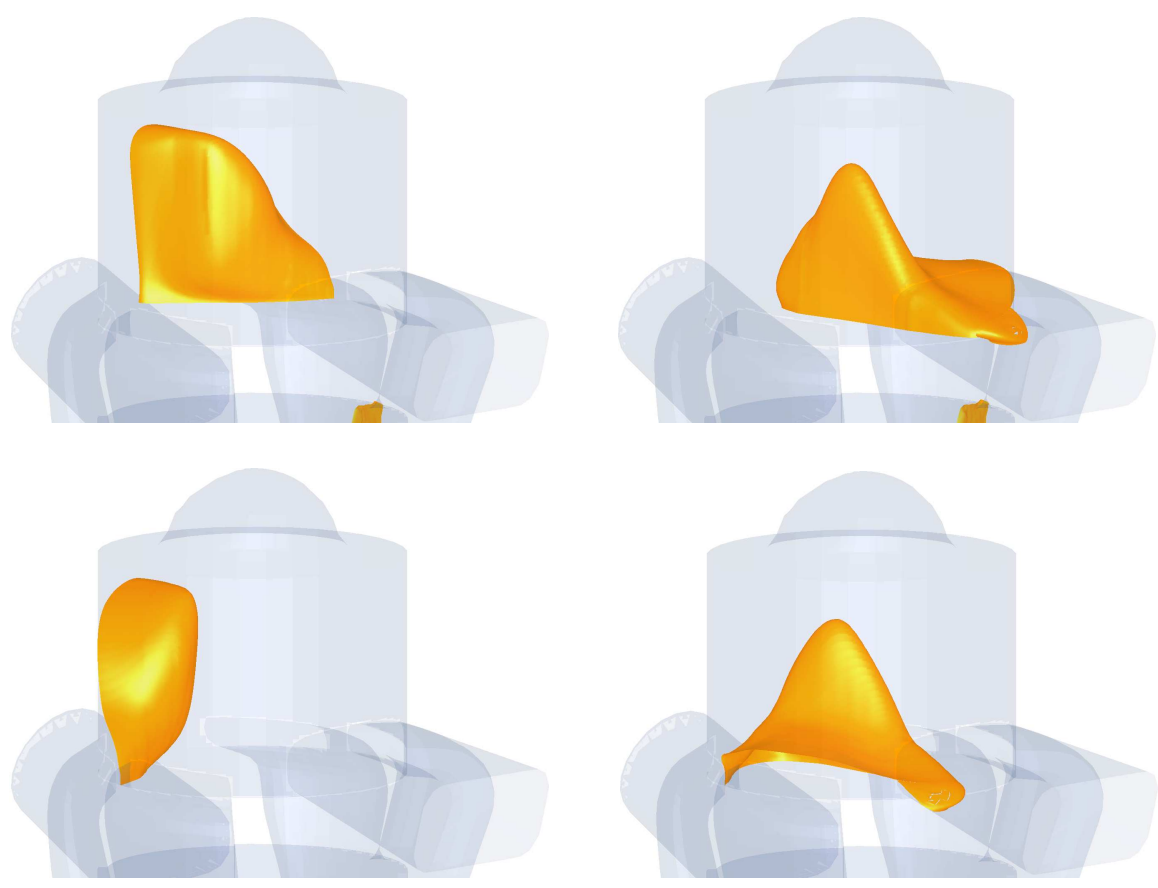

Figure 10.11.3: Fresh-gas tracking: shown is the iso-surface of 0.3 fresh-gas concentration coming from the single transfer ports. 


\section{Chapter 11}

\section{Summary and Outlook}

\subsection{Conclusion}

A software package has been implemented, which is able to efficiently simulate and analyze the viscous flow through a time-dependent two-stroke engine geometry.

The used numerical scheme is based on the Navier-Stokes equations, which describe a viscous time-dependent fluid and include thermal conductivity. In order to apply this scheme to the real-world problem of the two-stroke engine in motion, certain mesh-related algorithms had to be realized. It is very difficult to represent the complex geometry of the engine by one single mesh. Thus, the assembling of the mesh from several partial meshes is made possible by the grid merging technique. The piston motion is realized using the snapper algorithm. This algorithm was extended to handle the lower part of the piston as well. The rotational motion of the crankshaft is treated with the help of the curved interface method.

Because an accurate simulation has a big need for resources, it was essential to incorporate several techniques to reduce the computational time. A temporally consistent adaptive local time-stepping method is very efficient if meshes with different size elements are used, as is the case in real-world applications. The dynamic local mesh adaption with all the special mesh considerations, as the grid merging, the moving parts of the mesh, and a boundary fitting of refined elements, saves computational time by refining elements in regions where a higher resolution is needed. The temporal convergence to a periodical solution is accelerated by an enhanced initial data approach. Finally, the software was parallelized for the shared memory architecture using the OpenMP standard. Dynamic load balancing is achieved by an extended partitioning algorithm to account for the mesh manipulating routines. The different parts of the developed software have been rigorously validated on several test problems. The efficiency of the local time-stepping, the adaptive mesh refinement, and the parallelization have been proved by the simulation of an existing two-stroke engine.

Three important questions of the engineer have been investigated with the resulting software package. The simulation has been analyzed by the developed visualization techniques, and the characteristic exhaust data has been presented.

Thus, a software tool has been created that can be used to make an in depth analysis of the flow through a given two-stroke engine geometry within a reasonable 
time. It is also possible to quantitatively compare two or more geometries regarding their efficiency and exhaust characteristics.

\subsection{Further Steps}

There are several ways to further improve the quality of the simulations. What can already be included in the simulation, is the treatment of ring crevice volumes and rounded port corners (cf. [ARB92, RK97]). If the engine geometry incorporates these features, the finer meshes can be constructed accordingly. No changes need to be made in the actual software.

To enhance the properties of the two-stroke engine, further studies of the impact of an asymmetrical transfer port, especially in its upper part, can be conducted. For this analysis, the software can be used without modification.

One further promising approach to reduce the short-circuiting in the scavenge process is the "layering" of the fresh gas supply. At the onset of the scavenge process, pure air displaces the exhaust gas. Only later in the process the fuel charged fresh gas enters the cylinder, thus decreasing the possibility of a short-circuit flow. Two possibilities are thinkable to integrate this approach into the simulation. The underlying engine geometry could be meshed to incorporate the supply duct of fresh air to the transfer ports, or the composition of the gas within the transfer ports could be modified prior to transfer port opening. An adaption of the code is easily feasible.

To increase the accuracy of the numerical (discrete) approximation of the (continuous) Navier-Stokes equations, new higher order methods have been developed, namely the Discontinuous Galerkin methods, which seem to be very promising, regarding their efficiency. But higher order schemes are more time consuming than first order schemes on the same mesh. Their advantage is the accelerated convergence on finer meshes. But, generally, a certain mesh size has to be reached in order to experience the improved accuracy of higher order schemes (cf. Paragraph 9.1.2 on page 114 and [KK00, HK02]). This is especially true for calculations with the complex mesh routines that need to be raised to this higher order as well, which adds to the cost of such an approach.

A further extension of the used numerical scheme would be the application of a turbulence model (cf. e.g. [Klö92]). As the computational power is, despite our accelerating methods, far from performing a direct numerical simulation (DNS) of the small scale turbulences (at Reynolds numbers as high as $10^{5}$ in our application), a turbulence model would account for these small scale effects on the flow. Unfortunately, up to now, no rigorous approach has been proposed to deal with this problem in an exhaustive manner. But nevertheless, to include a heuristic turbulence model would be an improvement for the accuracy of the simulation.

And finally, in order to extend the software towards a tool for analyzing the combustion process, and optimizing the combustion chamber, a detailed threedimensional combustion model could be integrated. Even though this is a difficult task due to the different time scales in combustion processes, this would increase the versatility of this software package even more. 


\section{Appendix A}

\section{Tables}

Table A.1: Compression profile: $L^{1}$-error and EOC evolution on the different meshes

\begin{tabular}{|rlcr|}
\hline elements in x-direction & mesh width & $L^{1}$-error of density $\rho$ & EOC of $\rho$ \\
\hline \hline 100 & 0.2 & $1.5361 \cdot 10^{-1}$ & \\
200 & 0.1 & $9.6584 \cdot 10^{-2}$ & 0.6694 \\
400 & 0.05 & $5.6463 \cdot 10^{-2}$ & 0.7745 \\
800 & 0.025 & $3.0960 \cdot 10^{-2}$ & 0.8669 \\
1600 & 0.0125 & $1.5855 \cdot 10^{-2}$ & 0.9655 \\
3200 & 0.00625 & $7.3951 \cdot 10^{-3}$ & 1.1003 \\
6400 & 0.003125 & $3.1597 \cdot 10^{-3}$ & 1.2268 \\
12800 & 0.0015625 & $1.6020 \cdot 10^{-3}$ & 0.9799 \\
\hline
\end{tabular}

Table A.2: Shock tube: $L^{1}$-error and EOC evolution on the different meshes

\begin{tabular}{|rlcr|}
\hline elements in x-direction & mesh width & $L^{1}$-error of density $\rho$ & EOC of $\rho$ \\
\hline \hline 100 & 0.002 & $3.3014 \cdot 10^{-3}$ & \\
200 & 0.001 & $2.1096 \cdot 10^{-3}$ & 0.6461 \\
400 & 0.0005 & $1.3332 \cdot 10^{-3}$ & 0.6620 \\
800 & 0.00025 & $8.3412 \cdot 10^{-4}$ & 0.6766 \\
1600 & 0.000125 & $5.2772 \cdot 10^{-4}$ & 0.6605 \\
3200 & 0.0000625 & $3.2967 \cdot 10^{-4}$ & 0.6788 \\
6400 & 0.00003125 & $2.0860 \cdot 10^{-4}$ & 0.6603 \\
12800 & 0.000015625 & $1.3254 \cdot 10^{-4}$ & 0.6543 \\
\hline
\end{tabular}


Table A.3: Nozzle flow: $L^{1}$-error and EOC evolution on the different meshes

\begin{tabular}{|rlcc|}
\hline elements in xy-direction & mesh width & $L^{1}$-error of density $\rho$ & EOC of $\rho$ \\
\hline \hline 1000 & 0.002 & $1.545 \cdot 10^{-3}$ & \\
2000 & 0.001414 & $1.086 \cdot 10^{-3}$ & 0.9613 \\
4000 & 0.001 & $7.409 \cdot 10^{-4}$ & 1.1030 \\
8000 & 0.0007071 & $5.242 \cdot 10^{-4}$ & 0.9983 \\
16000 & 0.0005 & $3.525 \cdot 10^{-4}$ & 1.1444 \\
32000 & 0.0003536 & $2.461 \cdot 10^{-4}$ & 1.0370 \\
64000 & 0.00025 & $1.617 \cdot 10^{-4}$ & 1.2120 \\
128000 & 0.0001768 & $1.104 \cdot 10^{-4}$ & 1.1000 \\
256000 & 0.000125 & $7.699 \cdot 10^{-5}$ & 1.0410 \\
\hline
\end{tabular}

Table A.4: Poiseuille flow: $L^{1}$-error and EOC evolution on the different meshes

\begin{tabular}{|rrcc|}
\hline elements in y-direction & mesh width & $L^{1}$-error of velocity $v_{1}$ & EOC of $v_{1}$ \\
\hline \hline 12 & $4 \cdot 10^{-6}$ & $2.389 \cdot 10^{-4}$ & \\
25 & $2 \cdot 10^{-6}$ & $1.927 \cdot 10^{-4}$ & 0.3096 \\
50 & $1 \cdot 10^{-6}$ & $1.303 \cdot 10^{-4}$ & 0.5654 \\
100 & $5 \cdot 10^{-7}$ & $9.230 \cdot 10^{-5}$ & 0.4969 \\
200 & $2.5 \cdot 10^{-7}$ & $7.132 \cdot 10^{-5}$ & 0.3720 \\
\hline
\end{tabular}

Table A.5: Couette flow: $L^{1}$-error and EOC evolution on the different meshes

\begin{tabular}{|rrcc|}
\hline elements in y-direction & mesh width & $L^{1}$-error of velocity $v_{1}$ & EOC of $v_{1}$ \\
\hline \hline 12 & $4 \cdot 10^{-6}$ & $3.444 \cdot 10^{-5}$ & \\
25 & $2 \cdot 10^{-6}$ & $1.856 \cdot 10^{-5}$ & 0.8918 \\
50 & $1 \cdot 10^{-6}$ & $9.661 \cdot 10^{-6}$ & 0.9419 \\
100 & $5 \cdot 10^{-7}$ & $5.525 \cdot 10^{-6}$ & 0.8061 \\
200 & $2.5 \cdot 10^{-7}$ & $3.265 \cdot 10^{-6}$ & 0.7589 \\
\hline
\end{tabular}


Table A.6: Blasius plate: $L^{1}$-error and EOC evolution on the different meshes

\begin{tabular}{|rrcc|}
\hline elements in y-direction & mesh width & $L^{1}$-error of $v_{1}$ & EOC of $v_{1}$ \\
\hline \hline 15 & $1.6 \cdot 10^{-4}$ & $6.276 \cdot 10^{-3}$ & \\
30 & $8 \cdot 10^{-5}$ & $3.396 \cdot 10^{-3}$ & 0.8860 \\
60 & $4 \cdot 10^{-5}$ & $1.736 \cdot 10^{-3}$ & 0.9681 \\
120 & $2 \cdot 10^{-5}$ & $9.689 \cdot 10^{-4}$ & 0.8411 \\
240 & $1 \cdot 10^{-5}$ & $8.077 \cdot 10^{-4}$ & 0.2626 \\
\hline & & & \\
\hline elements in y-direction & mesh width & $L^{1}$-error of $v_{2}$ & EOC of $v_{2}$ \\
\hline \hline 15 & $1.6 \cdot 10^{-4}$ & $1.855 \cdot 10^{-5}$ & \\
30 & $8 \cdot 10^{-5}$ & $1.254 \cdot 10^{-5}$ & 0.5647 \\
60 & $4 \cdot 10^{-5}$ & $1.647 \cdot 10^{-5}$ & -0.3936 \\
120 & $2 \cdot 10^{-5}$ & $1.548 \cdot 10^{-5}$ & 0.0902 \\
240 & $1 \cdot 10^{-5}$ & $2.728 \cdot 10^{-5}$ & -0.8180 \\
\hline
\end{tabular}

\begin{tabular}{|rrrr|}
\hline elements in y-direction & mesh width & $L^{1}$-error of $T$ & EOC of $T$ \\
\hline \hline 15 & & $5.846 \cdot 10^{-4}$ & \\
30 & $8 \cdot 10^{-5}$ & $4.726 \cdot 10^{-4}$ & 0.3070 \\
60 & $4 \cdot 10^{-5}$ & $2.691 \cdot 10^{-4}$ & 0.8121 \\
120 & $2 \cdot 10^{-5}$ & $1.628 \cdot 10^{-4}$ & 0.7254 \\
240 & $1 \cdot 10^{-5}$ & $1.117 \cdot 10^{-4}$ & 0.5437 \\
\hline
\end{tabular}

Table A.7: Travelling wave: $L^{1}$-error and EOC evolution on the different meshes

\begin{tabular}{|rrcr|}
\hline elements in x-direction & mesh width & $L^{1}$-error of pressure $p$ & EOC of $p$ \\
\hline \hline 200 & $2 \cdot 10^{-6}$ & $2.791 \cdot 10^{-1}$ & \\
400 & $1 \cdot 10^{-6}$ & $1.420 \cdot 10^{-1}$ & 0.97542 \\
800 & $5 \cdot 10^{-7}$ & $7.115 \cdot 10^{-1}$ & 0.99655 \\
1600 & $2.5 \cdot 10^{-7}$ & $3.559 \cdot 10^{-2}$ & 0.99953 \\
3200 & $1.25 \cdot 10^{-7}$ & $1.775 \cdot 10^{-2}$ & 1.00388 \\
6400 & $6.25 \cdot 10^{-8}$ & $1.120 \cdot 10^{-2}$ & 0.66425 \\
12800 & $3.125 \cdot 10^{-8}$ & $6.905 \cdot 10^{-3}$ & 0.69759 \\
\hline
\end{tabular}


Table A.8: Sudden expansion: massflow evolution on the different meshes

\begin{tabular}{|rccc|}
\hline ref. level & mesh width & massflow ex. & massflow ex. bnd. \\
\hline \hline 0 & .0033333 & $4.216 \cdot 10^{-3}$ & $4.216 \cdot 10^{-3}$ \\
1 & .0016667 & $4.453 \cdot 10^{-3}$ & $4.453 \cdot 10^{-3}$ \\
2 & .0008333 & $4.654 \cdot 10^{-3}$ & $4.660 \cdot 10^{-3}$ \\
3 & .0004167 & $4.797 \cdot 10^{-3}$ & $4.797 \cdot 10^{-3}$ \\
4 & .0002083 & $4.886 \cdot 10^{-3}$ & $4.883 \cdot 10^{-3}$ \\
\hline
\end{tabular}

ref. level mesh width massflow vel.sp. massflow no vel.sp.

\begin{tabular}{|llll|}
\hline \hline 0 & .0033333 & $4.403 \cdot 10^{-3}$ & $3.455 \cdot 10^{-3}$ \\
1 & .0016667 & $4.590 \cdot 10^{-3}$ & $3.927 \cdot 10^{-3}$ \\
2 & .0008333 & $4.646 \cdot 10^{-3}$ & $4.499 \cdot 10^{-3}$ \\
3 & .0004167 & $4.854 \cdot 10^{-3}$ & $4.687 \cdot 10^{-3}$ \\
4 & .0002083 & $4.919 \cdot 10^{-3}$ & $4.830 \cdot 10^{-3}$ \\
\hline
\end{tabular}




\section{Appendix B}

\section{The Material Properties}

Table B.1: Material properties: physical constants (taken from [AdP01])

\begin{tabular}{|cc|}
\hline name & value \\
\hline \hline Avogadro's number $N_{A}$ & $6.02214 \cdot 10^{23} \frac{1}{\mathrm{~mol}}$ \\
universal gas constant $\mathcal{R}$ & $8.31451 \frac{\mathrm{J}}{\mathrm{mol} \mathrm{K}}$ \\
\hline
\end{tabular}

Table B.2: Material properties: characteristic material constants (taken from [Vdi97])

\begin{tabular}{|ccccc|}
\hline material & molar mass & crit. & crit. & crit. real \\
& $M$ & $\begin{array}{c}\text { temperature } \\
T_{c}\end{array}$ & $\begin{array}{c}\text { pressure } \\
p_{c}\end{array}$ & $\begin{array}{c}\text { gas factor } \\
Z_{c}\end{array}$ \\
& {$[\mathrm{~kg} / \mathrm{kmol}]$} & {$[\mathrm{K}]$} & {$[\mathrm{bar}]$} & \\
\hline \hline air & 28.96 & 132.5 & 37.7 & \\
nitrogen $\mathrm{N}_{2}$ & 28.013 & 126.2 & 33.9 & 0.290 \\
oxygen $\mathrm{O}_{2}$ & 31.999 & 154.6 & 50.4 & 0.288 \\
water $\mathrm{H}_{2} \mathrm{O}$ & 18.015 & 647.3 & 221.2 & 0.235 \\
carbon dioxide $\mathrm{CO}_{2}$ & 44.010 & 304.1 & 73.8 & 0.274 \\
\hline
\end{tabular}


Table B.3: Material properties of air at standard temperature and pressure (STP) (see Table B.5) (taken from [Vdig7])

\begin{tabular}{|cc|}
\hline property & value \\
\hline \hline heat capacity at constant pressure $c_{p}$ & $1.005 \frac{\mathrm{kJ}}{\mathrm{kg} \mathrm{K}}$ \\
viscosity $\eta$ & $1.848 \cdot 10^{-5} \frac{\mathrm{kg}}{\mathrm{s} \mathrm{m}}$ \\
thermal conductivity $\lambda$ & $2.606 \cdot 10^{-2} \frac{\mathrm{W}}{\mathrm{K} \mathrm{m}}$ \\
\hline
\end{tabular}

Table B.4: Material properties: specific heat capacity at constant pressure $c_{p}$ in [kJ/kg K] (taken from [Vdig7])

\begin{tabular}{|ccccccccccc|}
\hline material & \multicolumn{10}{c|}{ temperature in ${ }^{\circ} \mathrm{C}$} \\
& 0 & 25 & 100 & 200 & 300 & 400 & 500 & 1000 & 1500 & 2000 \\
\hline \hline air & 1.004 & 1.005 & 1.009 & 1.022 & 1.043 & 1.07 & 1.09 & 1.18 & 1.23 & 1.26 \\
nitrogen $\mathrm{N}_{2}$ & 1.039 & 1.039 & 1.04 & 1.05 & 1.07 & 1.09 & 1.12 & 1.22 & 1.27 & 1.30 \\
oxygen $\mathrm{O}_{2}$ & 0.914 & 0.917 & 0.934 & 0.963 & 0.992 & 1.026 & 1.05 & 1.12 & 1.16 & 1.20 \\
water $\mathrm{H}_{2} \mathrm{O}$ & 1.859 & 1.864 & 1.889 & 1.940 & 2.00 & 2.064 & 2.13 & 2.48 & 2.75 & 2.93 \\
carb. dioxide $\mathrm{CO}_{2}$ & 0.827 & 0.851 & 0.919 & 0.997 & 1.061 & 1.114 & 1.159 & 1.294 & 1.319 & 1.352 \\
\hline
\end{tabular}

Table B.5: Material properties: used European standard temperature and pressure (STP) (taken from [AdP01])

\begin{tabular}{|cc|}
\hline variable & value \\
\hline \hline pressure $p$ & $101325 \mathrm{~Pa}$ \\
temperature $T$ & $293.15 \mathrm{~K}$ \\
density $\rho$ & $1.204 \frac{\mathrm{kg}}{\mathrm{m}^{3}}$ \\
\hline
\end{tabular}




\section{Appendix C}

\section{The Software Package engine_flow}

The software package engine_flow consists of the main programs engine_flow and viewer. The program engine_flow is the implementation of the numerical algorithms described in this thesis. It simulates the flow through a two-stroke engine. The viewer is the tool to visually analyze the results from the engine_flow program. It is an extended interface to the graphics library GRAPE (see [Gra99]). Furthermore, there are the utility programs startdata to generate the enhanced initial data for the next finer mesh (as described in Section 6.3), the program symmetrizer to calculate a symmetrical transfer port mesh, and the tool mesh_check to verify the integrity of a computational mesh.

The engine_flow user manual of the software package can be found on the enclosed CD-ROM in the directory engine/manual/ or is available from Www.mathematik.uni-freiburg.de/IAM/homepages/trescher/, as the software package itself.

The engine_flow reference manual is a description of the source code and is also available from the above mentioned sources.

\section{engine_flow}

The source files of the main program engine_flow can be found in the directory engine/engine_flow/sources/ on the enclosed CD-ROM. We now describe briefly the important C-sources:

- engine_general.h: header-file needed for all programs in the package.

- engine_flow.h: header-file needed only for the program engine_flow.

- engine_flow.c: $\operatorname{main}()$ and the time-step iteration.

- local_dt.c: the calculation of the main numerical scheme and all other algorithms depending on the local $\Delta t$ algorithm.

- convect.c: computation of the numerical convective flux. 
- viscous.c: computation of the numerical viscous flux.

- grid_merge.c: the grid merging algorithm.

- merge_lowlevel.c: the routines to manage the grid merging structure.

- piston.c: the piston motion algorithm.

- rotate_crank.c: the crankshaft rotation algorithm.

- gas.c: calculation of the fresh charge flow and exhaust gas flow in and out of the cylinder.

- refine.c: the routines for the refinement of an element.

- coarsen.c: the routines for the coarsening of an element.

- gas_properties.c: computation of the temperature-dependent material properties.

- bnd_data.c: the routine for the parsing of the time-dependent boundary condition data.

- problem.c: problem-specific routines such as setting of initial data and treatment of the boundary conditions.

- combustion.c: implementing the (very basic) combustion model.

- geom_sizes.c: routines for the determination of the element sizes.

- in out.c: the second level input/output routines.

- files.c: the file input/output routines.

- parallel.c: the additional routines for the parallel partitioning algorithm.

For the usage of the program additional data is required (example files are located in the directory engine/engine_flow/:

- All parameters are given in a parameter-file (to be found in the subdirectory engine/engine_flow/param/).

- There the location of the needed meshes is specified.

- Also the path of the optional data file for the time-dependent boundary condition data is given.

The program generates several data files for the analyzation of the flow:

- every $<$ DEG4GAS $>$ degrees crank angle atdc, specified in the parameter file, the fresh charge flow and exhaust gas flow in and out of the cylinder is analyzed and printed into several files, whose location if determined by $<$ GASFILE $>$. 
- every <TIMEDEG4WRITE > degrees crank angle atdc, also specified in the parameter file, the complete solution of the actual time-step is printed into the file $<$ GASFILE $>$ xxxxx.xdat.

- additionally, the file $<$ CONTFILE $>$ is written to store information on the grid merging structure. This speeds up successive runs of the simulation with the same mesh.

\section{viewer}

The C-sources and header files of the vizualization tool viewer are located in the directory engine/viewer/sources/. They use mainly the same routines as the engine_flow program. Additional sources include:

- viewer.h: header needed for the program viewer.

- viewer.c: main() and the GRAPE control interface.

- mesh.c: the GRAPE mesh interface.

- bnd_disp.c: routine for the display of the boundary type of elements.

- level_disp.c: display routine for the local $\Delta t$-level of the elements (cf. Figure 10.4.1 on page 174).

- partition_disp.c: display routine for the parallel partitions of the elements.

- ghost_disp.c: display routine for the critical elements of a parallel partition.

- clip_bnd_disp.c: display routine for a clipped opaque geometry view.

In order to use the viewer software on a standard Linux environment no recompilation is necessary. Just use the precompiled binary viewer in the directory engine/viewer/. For the compilation of the viewer the GRAPE-library has to be present on the system. This has to be obtained from the Department of Applied Mathematics of the University of Freiburg (just e-mail treschermathematik.unifreiburg.de). It is generated for a standard Linux distribution. Other builds are available upon request (not Windows ${ }^{\circledR}$ ).

As the usage of the GRAPE interface is sometimes not intuitive, a closer look into the enclosed GRAPE-manual, located in engine/viewer/grape_manual is encouraged.

\section{utility tools}

The utility programs startdata, symmetrizer, and mesh_check are located in the directories engine/utilities/startdata, engine/utilities/symmetrizer/, and engine/utilities/mesh_check/ respectively. Their straightforward usage is explained in the enclosed manual. 


\section{List of Figures}

1.1.1 Typical applications of two-stroke engines. . . . . . . . . . . 2

2.2.1 Components of a two-stroke engine. . . . . . . . . . . . . 10

2.2.2 Different phases in the cycle of a two-stroke engine. . . . . . . . . 11

2.3.1 Geometrical properties of the two-stroke engine. . . . . . . . . 13

2.3 .2 Position of the piston. . . . . . . . . . . . . . 14

2.4.1 Different port plan layouts of loop scavenged engines. . . . . . . 16

2.4.2 Handle-type and open-type transfer ports. . . . . . . . . . . . . 16

2.4.3 Horizontal and vertical port orientations. . . . . . . . . . . . 17

2.4.4 Cross scavenging and uniflow scavenging. . . . . . . . . . . . 17

2.4.5 SR-TE diagram of the different simple scavenging models. . . . . . 19

2.4.6 Perfect displacement and perfect mixing. . . . . . . . . . . . . 19

2.5.1 Draft of the theoretical Otto Cycle. . . . . . . . . . . . 20

2.7.1 SR-SE, SR-TE, and SR-purity diagrams. . . . . . . . . 24

2.7.2 Different flow patterns according to JANTE. . . . . . . . . . . . . 24

2.7 .3 Vertical velocity boundary . . . . . . . . . . . . . . 25

2.7 .4 Deviation of the real flow angle. . . . . . . . . . . 26

2.7.5 Piston at closure of exhaust port, at tdc and squish velocity. . . . 26

3.8.1 Temperature-dependency of material properties. . . . . . . . . . 40

4.1 .1 Mesh-related notations. . . . . . . . . . . . . . . . 46

4.2.1 Hanging knots in a locally refined mesh. . . . . . . . . . . . . . . 47

4.5.1 Gradient calculation. . . . . . . . . . . . . . . . 54

4.7.1 Dirichletand Von Neumann boundary treatment. . . . . . . . . . 56

4.10 .1 Flowchart of the main numerical scheme. . . . . . . . . . . . 64

5.1 .1 Overset and patched grids. . . . . . . . . . . . 66

5.1 .2 Detailed view of a patched grid and boundary facet. . . . . . . . 68

5.1 .3 The virtual splitting method. . . . . . . . . . . . . 68

5.1.4 Flowchart of the grid merging algorithm. . . . . . . . . . . 69

5.2.1 The snapper algorithm, moving, merging, and splitting. . . . . . . 74

5.2.2 Two different pistons: layering possible and impossible. . . . . . . 75

5.2.3 The neighboring cells get affected by the piston motion. . . . . . 76

5.2.4 Flowchart of the moving piston algorithm. . . . . . . . . . . 77

5.3.1 The fitted interface method. . . . . . . . . . . . . 79

5.3.2 Holes in the outer layer of the crankshaft mesh. . . . . . . . . . . 80

5.3.3 Round element faces in the crankcase and crankshaft meshes. . . . 82 
5.3.4 Swept volume of a face $S_{j l}$ at crankshaft rotation. . . . . . . 85

5.3.5 Flowchart of the rotating crankshaft algorithm. . . . . . . . 86

6.1.1 Time step levels. . . . . . . . . . . . . . . . . . . . . 88

6.1.2 Flowchart of the local time-step algorithm. . . . . . . . . . . . 90

6.2.1 Refinement and coarsening induced by piston motion. . . . . . . . 96

6.2.2 Refinement of Neighbors influenced by the piston motion. . . . . . 96

6.2.3 Flowchart of the mesh adaption algorithm. . . . . . . . . . . 98

8.2.1 Modeled crankcase volume and massflow condition at the inlet. . . 109

8.2.2 Window area fraction and pressure boundary condition. . . . . . . 109

8.2.3 Movement of the connecting rod. . . . . . . . . . . . . . . . 110

8.3.1 Quasi-2D mesh with and without connecting rod. . . . . . . . 111

8.4.1 Temporal convergence history. . . . . . . . . . . . . . . . 111

8.4.2 Structure of the flow. . . . . . . . . . . . . . . . 112

9.2.1 Compression profile: mesh and calculated density. . . . . . . . . . 115

9.2.2 Compression profile: steep-fronting. . . . . . . . . . . . . . 115

9.2.3 Compression profile: boundary conditions and domain $\Omega$. . . . . 117

9.2.4 Compression profile: density on the different meshes. . . . . . . . 118

9.2.5 Compression profile: $L^{1}$-error and EOC evolution. . . . . . . . . . 118

9.2.6 Shock tube: mesh and calculated density. . . . . . . . . . . . . 119

9.2.7 Shock tube: boundary conditions and domain $\Omega$. . . . . . . . . 120

9.2.8 Shock tube: exact density, velocity, and pressure. . . . . . . . . . . 121

9.2.9 Shock tube: the density profile along the tube. . . . . . . . . . . 121

9.2.10 Shock tube: $L^{1}$-error of the density and corresponding EOC. . . . 122

9.2.11 Nozzle flow: mesh and calculated density. . . . . . . . . . . . . . 122

9.2 .12 Nozzle flow: boundary conditions and domain $\Omega$. . . . . . . . . . 124

9.2.13 Nozzle flow: density profile along the nozzle. . . . . . . . . . . . 125

9.2.14 Nozzle flow: $L^{1}$-error of the density and corresponding EOC. . . . 125

9.2.15 Channel with a bump: mesh and computed Mach number. . . . . 126

9.2.16 Channel with a bump: boundary conditions and domain $\Omega$. . . . . 127

9.2.17 Channel with a bump: comparison of results (Mach number). . . . 128

9.2.18 Channel with a bump: comparison of results (Mach contours). . . 128

9.3.1 Poiseuille flow: mesh and computed Mach number. . . . . . . . . . 129

9.3.2 Poiseuille flow: boundary conditions and domain $\Omega$. . . . . . . 131

9.3.3 Poiseuille flow: temporal convergence. . . . . . . . . . . . . . . 132

9.3.4 Poiseuille flow: velocity profile. . . . . . . . . . . . . . . 132

9.3.5 Poiseuille flow: $L^{1}$-error and EOC. . . . . . . . . . . . . . . 132

9.3.6 Couette flow: mesh and computed Mach number. . . . . . . . . . 133

9.3.7 Couette flow: boundary conditions and domain $\Omega$. . . . . . . . 134

9.3.8 Couette flow: temporal convergence. . . . . . . . . . . . . . . 135

9.3.9 Couette flow: velocity profile. . . . . . . . . . . . . . 135

9.3.10 Couette flow: $L^{1}$-error and EOC. . . . . . . . . . . . . . 136

9.3.11 Blasius plate: mesh and computed temperature. . . . . . . . . 136

9.3.12 Blasius plate: the stream function $f$ and its derivatives. . . . . . . 138

9.3.13 Blasius plate: exact velocity and temperature profile. . . . . . . 138 
9.3.14 Blasius plate: boundary conditions and domain $\Omega$. . . . . . . 139

9.3.15 Blasius plate: temporal convergence. . . . . . . . . . . . . . . 140

9.3.16 Blasius plate: velocity and temperature profiles. . . . . . . . . . 141

9.3.17 Blasius plate: $L^{1}$-error and EOC. . . . . . . . . . . . . . . 141

9.3.18 Traveling wave: mesh and computed pressure. . . . . . . . . . . 142

9.3.19 Traveling wave: boundary conditions and domain $\Omega$. . . . . . . . 143

9.3.20 Traveling wave: pressure profile. . . . . . . . . . . . . . . . . 144

9.3.21 Traveling wave: $L^{1}$-error and EOC. . . . . . . . . . . . . . . 144

9.4.1 Sudden expansion: mesh and computed Mach number. . . . . . . 145

9.4.2 Sudden expansion: boundary conditions and domain $\Omega$. . . . . . . 147

9.4.3 Sudden expansion: initial pressure distribution $p_{0}(x) \ldots \ldots$. . . . 147

9.4.4 Sudden expansion: the three different types of meshes. . . . . . . 148

9.4.5 Sudden expansion: convergence of the total massflow. . . . . . . . 149

9.4.6 Forward facing step: mesh and computed density. . . . . . . . . . 149

9.4.7 Forward facing step: boundary conditions and domain $\Omega$. . . . . 151

9.4.8 Forward facing step: influence of adaption at $t=0.5, t=1 \ldots 153$

9.4.9 Forward facing step: influence of adaption at $t=1.5, t=2 \ldots \ldots 154$

9.4.10 Forward facing step: influence of refinement constants. . . . . . . . 155

9.4.11 Forward facing step: influence of refinement level 2-4. . . . . . . 156

9.4.12 Forward facing step: influence of refinement level 5-7. . . . . . . 157

9.4 .13 Forward facing step: number of elements. . . . . . . . . . . . 158

9.4.14 Boundary fitting: comparison of Mach contours. . . . . . . . . . 158

9.4.15 Boundary fitting: comparison of Mach number plots. . . . . . . . 159

9.5.1 Exhaust pipe: mesh and computed pressure. . . . . . . . . . . 159

9.5.2 Exhaust pipe: boundary conditions and domain $\Omega$. . . . . . . . . 161

9.5.3 Exhaust pipe: convergence of the pressure profile. . . . . . . . . 161

9.5.4 Exhaust pipe: comparison of the pressure with the experiment. . . 162

9.5.5 Intake pipe: convergence of the pressure profile. . . . . . . . . 163

9.5.6 Intake pipe: comparison of the pressure with the experiment. . . . 164

9.5.7 Otto Cycle: mesh and computed temperature with velocity. . . . . 164

9.5.8 Otto Cycle: boundary conditions anddomain $\Omega . \ldots 165$

9.5.9 Otto Cycle: influence of temperature-dependency. . . . . . . . 166

10.1.1 Meshes: different parts and 250000 element mesh. . . . . . . . . . 168

10.1.2 Meshes: details of the mesh generation. . . . . . . . . . . . . 169

10.2.1 Port areas by piston control of the different ports. . . . . . . . . . 171

10.4.1 Parallelization: local time-step level and partitioning. . . . . . . . 174

10.4.2 Parallelization: speed-up, efficiency, and scalability. . . . . . . 175

10.6.1 The temporal convergence with and without enhanced initial data. 177

10.6.2 Convergence of the flow structure. . . . . . . . . . . . 178

10.6.3 Convergence of the scavenge ratio-trapping efficiency graph. . . . 179

10.6.4 Comparison with measured pressure data. . . . . . . . . . . 180

10.6.5 Visualization of the flow structure: Particle traces in the cylinder. 181

10.6.6 Visualization of the flow structure: Particle traces in the crankcase. 181

10.6.7 Visualization of the flow structure: iso-surfaces at $150^{\circ}$ crank angle. 182

10.6.8 Visualization of the flow structure: iso-surfaces at $180^{\circ}$ crank angle. 183

10.6.9 Visualization of the flow structure: iso-surfaces at $220^{\circ}$ crank angle. 184 
10.6.10 Visualization of the flow structure: Objects moving with the flow. 185 10.6.11 Visualization of the flow structure: horizontal clipping planes. . . 187 10.6.12 Visualization of the flow structure: vertical clipping planes. . . . . 188 10.6.13 Characteristical diagrams: SR-purity, SR-SE, and SR-TE. . . . . 189 10.6.14 Characteristical diagrams: massflow at transfer port and outlet. . 189 10.6.15 Jante test: velocity field with fresh charge concentration. . . . . . 190 10.6.16 Sharp vertical velocity boundary: velocity field. . . . . . . . . . . . 191 10.6.17 Deviation angles: horizontal and vertical deviation. . . . . . . . . 191 10.6.18 Deviation angles: temporal dependency. . . . . . . . . . . . . . . 192 10.6.19 Squish velocity: radial velocity plot. . . . . . . . . . . . . . . . 192 10.6.20 Squish velocity: velocity vectors. . . . . . . . . . . . . . . 193

10.7.1 Efficiency of the local time-stepping: $\Delta t$-level distribution. . . . . 193

10.7.2 Efficiency of the local time-stepping: time and speed-up. . . . . . 194

10.7.3 Efficiency of the local mesh adaption: refinement of the mesh. . . 194

10.7.4 Efficiency of the local mesh adaption: number of elements. . . . . 195

10.8.1 Call graph with execution time information. . . . . . . . . . . 196

10.9.1 Effect of crankshaft rotation: Particle traces in the non-rotating crankcase. . . . . . . . . . . . . . . . . 197

10.9.2 Effect of crankshaft rotation: difference in the SR-TE graph. . . . 198

10.9.3 Effect of crankshaft rotation: transfer port shadowing. . . . . . . . 198

10.10.1 Unsymmetrical transfer port: comparison of geometries. . . . . . . 199

10.10.2 Unsymmetrical transfer port: difference in the SR-TE graph. . . . 200

10.11.1 Fresh-gas tracking: massflow plot at the transfer port-cylinder. . . 201

10.11.2 Fresh-gas tracking: fresh-gas contours from each transfer port. . . 202

10.11.3 Fresh-gas tracking: iso-surface of fresh-gas from each transfer port. 202 


\section{List of Tables}

$2.1 \quad$ Notations in the context of the two-stroke engine . . . . . . . 9

$3.1 \quad$ Notations for the temperature-dependent material properties . . . . 40

$4.1 \quad$ Notations for the definition of the mesh structure . . . . . . . . 45

10.1 The different mesh families for the two-stroke engine simulation . 170

10.2 Geometrical data of the test engine. . . . . . . . . . . . . 171

10.3 Port timing for the test engine . . . . . . . . . . . 171

A.1 Compression profile: $L^{1}$-error and EOC evolution . . . . . . . 205

A.2 Shock tube: $L^{1}$-error and EOC evolution . . . . . . . . . . . 205

A.3 Nozzle flow: $L^{1}$-error and EOC evolution . . . . . . . . . . 206

A.4 Poiseuille flow: $L^{1}$-error and EOC evolution . . . . . . . . 206

A.5 Couette flow: $L^{1}$-error and EOC evolution . . . . . . . . . . . 206

A.6 Blasius plate: $L^{1}$-error and EOC evolution . . . . . . . . . . 207

A.7 Travelling wave: $L^{1}$-error and EOC evolution . . . . . . . . . 207

A.8 Sudden expansion: massflow evolution . . . . . . . . . . . 208

B.1 Material properties: physical constants. . . . . . . . . . . 209

B.2 Material properties: characteristic material constants . . . . . . 209

B.3 Material properties of air . . . . . . . . . . . . . . . 210

B.4 Material properties: specific heat capacity . . . . . . . . . . . 210

B.5 Material properties: standard temperature and pressure (STP) . . 210 



\section{Bibliography}

[AH92] G. Adam and O. Hittmair. Wärmetheorie, Vieweg-Verlag (Vierte Auflage, 1992).

[ARB92] A.A. Amsden, P.J. O'Rourke, and T.D. Butler. Comparisons of computed and measured three-dimensional velocity fields in a motored twostroke engine, SAE Paper No. 920418, Society of Automotive Engeneers, Warrendale, Pa. (1992).

[AdP01] P.W. Atkins and J. de Paula. Physical Chemistry, Oxford University Press, Oxford (Seventh Edition, 2001).

[Bän89] E. Bänsch. Local mesh refinement in 2 and 3 dimensions, Report 6, SFB 256, Bonn (1989).

[Bän91] E. Bänsch. An adaptive finite-element strategy for the threedimensional time-dependent Navier-Stokes equations, J. Comp. and Appl. Math., 36: 3-28 (1991).

[BLN79] C. Bardos, A.Y. Leroux, and J.C. Nedelec. First Order Quasilinear Equations with boundary conditions, Comm. in Part. Diff. Equ., 4(9), 1017-1034 (1979).

[Be00] J. Becker. Entwicklung eines effizienten Verfahrens zur Lösung hyperbolischer Differentialgleichungen, Doctoral Dissertation, Mathematisches Institut, Freiburg (2000).

[BPR01] J. Becker, T. Preuser, and M. Rumpf. PDE methods in flow simulation post processing, Comput. Visual. Sci., to appear (2001).

[B196] G.P. Blair. Design and Simulation of Two-Stroke Engines, Society of Automotive Engineers, Inc. (1996).

[Cha98] S. Champier. Error estimates for the approximate solution of a nonlinear hyperbolic equation with source term given by the finite volume scheme, Preprint, CNRS UMR 5585, Université de Saint-Etienne, available from WWW: numerix.univ-lyon1.fr/publis/publiv/1997/ publis.html (1998). 
[CH94] G. Chesshire and W.D. Henshaw. A scheme for conservative interpolation on overlapping grids, SIAM J. Sci. Comput., Vol. 15, No. 4, 819-845 (1994).

[CP98] F. Coquel and B. Perthame. Relaxation of energy and approximate Riemann solvers for general pressure laws in fluid dynamics, SIAM J. Numer. Anal. 35, no. 6, 2223-2249 (1998).

[Ded03] A. Dedner. Solving the system of radiation magnetohydrodynamics for solar physical simulations in 3d, Doctoral Dissertation, Mathematisches Institut, Freiburg (2003).

[DRSW04] A. Dedner, C. Rohde, B. Schupp, and M. Wesenberg. A parallel, load balanced MHD Code on locally adapted, unstructured grids in 3D, Comput. Visual. Sci. 7/2, 79-96 (2004).

[DP90] I. Demirdžić and M. Perić. Finite volume method for prediction of fluid flow in arbitrarily shaped domains with moving boundaries, Int. J. Num. Meth. in Fluids, 10: 771-790 (1990).

[EKS99] A. Egelja, D. Kröner, and R. Schwörer. Adaptive grids for time dependent conservation laws: theory and applications in CFD. High performance scientific and engineering computing (Munich, 1998), 25-37, Lect. Notes Comput. Sci. Eng., 8, Springer, Berlin (1999).

[EKS+98] A. Egelja, D. Kröner, R. Schwörer, N. Lanson, M. Mancip, and J.P. Vila. Combined finite volume and smoothed particle method. Numerical flow simulation, I (Marseille, 1997), 50-74, Notes Numer. Fluid Mech., 66, Vieweg, Braunschweig (1998).

[FP97] J. Ferziger and M. Perić. Computational Methods for Fluid Dynamics, Springer-Verlag, Berlin (1997).

[FR02] H. Freistühler and Ch. Rohde. Numerical computation of viscous profiles for hyperbolic conservation laws. J. Math. Comput. 71, No.239, 1021-1042 (2002).

[Fuj95] K. Fujii. Unified zonal method based on the fortified solution algorithm, J. Comput. Phys., 118, 92-108 (1995).

[FBLV04] C. Fumeaux, D. Baumann, P. Leuchtmann, and R. Vahldieck. A generalized local time-step scheme for efficient FVTD simulations in strongly inhomogeneous meshes, IEEE Trans. Microwave Theory \& Tech., vol. 52, No. 3, 1067-1076 (2004).

[GKR93] M. Geiben, D. Kröner, and M. Rokyta. A Lax-Wendroff type theorem for cell centered, finite volume schemes in 2D, Preprint 278, SFB 256, Bonn (1993). 
[Geß94] T. Geßner. Zeitabhängige Adaption für Finite Volumen Verfahren höherer Ordnung am Beispiel der Euler-Gleichungen der Gasdynamik, Institut für Angewandte Mathematik, Diplomarbeit, Universität Bonn (1994).

[Geß01] T. Geßner. Dynamic mesh adaption for supersonic combustion waves modeled with detailed reaction mechanisms, Doctoral Dissertation, Mathematisches Institut, Freiburg (2001).

[GK01a] T. Geßner and D. Kröner. Dynamic mesh adaption for supersonic reactive flow, Proceedings: Hyperbolic problems: Theory, Numerics, Applications, Eighth international conference, Magdeburg 2000, 415-424 (2001).

[GK01b] T. Geßner and D. Kröner. Godunov type methods on unstructured grids and local mesh refinement, in: Godunov Methods Theory and Applications, New York, 527-548. Ed.: E.F. Toro (2001).

[GR96] E. Godlewski and P.-A. Raviart. Numerical Approximation of Hyperbolic Systems of Conservation Laws, Springer-Verlag, New York (1996).

[Gra99] Grape-Team. GRAPE GRAphics Programming Environment manual, available from WWW: www.mathematik.uni-freiburg.de/IAM/ Research/grape/DOC/HTML/manual .html (1999).

[HK02] R. Herbin and D. Kröner (Hrsg.). Finite Volumes for Complex Applications III, Hermes Penton Science London (2002).

[Hir92a] C. Hirsch. Numerical Computation of internal and external Flows, Volume I: Fundamentals of Numerical Discretization, John Wiley \& Sons (1992).

[Hir92b] C. Hirsch. Numerical Computation of internal and external Flows, Volume II: Computational Methods for Inviscid and Viscous Flows, John Wiley \& Sons (1992).

[Hir74] C.W. Hirt. An arbitrary Lagrangian-Eulerian computing method for all flow speeds, J. Comp. Phys. 14: 227-253 (1974).

[HW92] C.J. Hwang and S.J. Wu. Global and local remeshing algorithms for compressible flows, J. Comput. Phys., 102: 98-113 (1992).

[Ice04] ICEM CFD HEXA, ICEM CFD Engineering, (2004).

[Jan68] A. Jante. Scavenging and other problems of two-stroke spark-ignition engines, SAE Paper No. 680468, Society of Automotive Engeneers, Warrendale, Pa. (1968). 
[Jen97] P. Jenny. On the numerical solution of the compressible Navier-Stokes equations for reacting and non-reacting gas mixtures, Doctoral Dissertation, ETH Zürich (1997).

[Kal96a] Y. Kallinderis. Domain partitioning and load balancing for parallel computation, in: Computational Fluid Dynamics, von Karman Institute for Fluid Dynamics, Lecture Series 1996-06 (1996).

[Kal96b] Y. Kallinderis. Grid adaptation by redistribution and local embedding, in: Computational Fluid Dynamics, von Karman Institute for Fluid Dynamics, Lecture Series 1996-06 (1996).

[KKKT03] L. Klassen, A. Klimmek, D. Kröner, and D. Trescher. Numerical optimization of scavenging in two-stroke engines with transfer ducts, an exhaust port and a moving piston, in W. Jäger, H.-J. Krebs, Mathematics, Key Technology for the Future, Springer (2003).

[KK00] L. Klassen and D. Kröner. Discretization of higher order for conservation laws on nonconformal unstructured rectangular grids, Manuskript (2000).

[KKS01] L. Klassen, D. Kröner, and Ph. Schott. Finite volume method on unstructed grids in 3D with applications to the simulation of gravity waves, submitted to Met. Atm. Phys. (2001).

[KKO02] R. Klöfkorn, D. Kröner, and M. Ohlberger. Local adaptive methods for convection dominated problems, to appear in: Int. J. Num. Meth. in Fluids (2002).

[Klö92] J. Klöker. Numerische Simulation einer dreidimensionalen, kompressiblen, reibungsbehafteten Strömung im Zylinder eines Modellmotors, Doctoral Dissertation, RWTH Aachen (1992).

[KM00] E. Krause and M. Meinke. CFD-applications on NEC SX-4, in E. Krause, W. Jäger (Eds), High performance computing in Science and Engineering, Springer (2000).

[Krö97] D. Kröner. Numerical Schemes for Conservation Laws, Verlag Wiley\&Teubner, Stuttgart (1997).

[KO00] D. Kröner and M. Ohlberger. A posteriori error estimates for upwind finite volume schemes for nonlinear conservation laws in multi dimensions. Math. Comp. 69: 25-39 (2000).

[LL95] L.D. Landau and E.M. Lifschitz. Theoretical Physics, Vol. VI, Pergamon Press, Oxford (1995). 
[LSG+94] L.J. Leep, G.S. Strumolo, V.L. Griaznov, S. Sengupta, A.M. Brohmer, and J. Meyer. CFD investigations of the scavenging process in a twostroke engine, SAE Paper No. 941929, Society of Automotive Engeneers, Warrendale, Pa. (1994).

[LeV90] R.J. LeVeque. Numerical Methods for Conservation Laws, Birkhäuser, Basel (1990).

[LS93] M.-S. Liou and C.J. Steffen. A new flux splitting scheme, J. Comput. Phys., 107: 23-39 (1993).

[MJ89] D.J. Mavriplis and A. Jameson. Multigrid solution of the Navier-Stokes equations on triangular meshes, AIAA Paper 89-0120, AIAA 27th Aerospace Sciences Meeting, Reno, (1989).

[Mit02] W. Mitianiec. Analysis of loop scavenging process in a smal power SI two-stroke engine, SAE Paper No. 2002-01-2181, Society of Automotive Engeneers, Warrendale, Pa. (2002).

[Mpi97] MPI-Team. MPI: A Message-Passing Interface Standard (1994), and MPI-2: Extensions to the Message-Passing Interface (1997), available from WWW: www.mpi-forum.org/docs/docs.html.

[Muz94] S. Muzaferija. Adaptive finite volume method for flow prediction using unstructured meshes and multigrid approach, Doctoral Dissertation, Department of Mechanical Engineering, University of London (1994).

[NTT94] H. Nessyahu, T. Tassa, and E. Tadmor. The convergence rate of Godunov type schemes, SIAM J. Numer. Anal., 32(1): 1-16 (1994).

[NORS97] R. Neubauer, M. Ohlberger, M. Rumpf, and R. Schwörer. Efficient visualization of large-scale data on hierarchical meshes, In Visualization in Scientific Computing '97, 125-137, Springer (1997).

[Ohl01] M. Ohlberger. A posteriori error estimate for finite volume approximations to singularly perturbed nonlinear convection-diffusion equations, Numer. Math. 87(4): 737-761 (2001).

[OR97] M. Ohlberger and M. Rumpf. Hierarchical and adaptive visualization on nested grids, Computing, Vol. 59, No. 4, 365-385 (1997).

[OR99] M. Ohlberger and M. Rumpf. Adaptive projection operators in multiresolution scientific visualization, IEEE Transactions on Visualization and Computer Graphics, Vol. 5 No. 1, 74-94 (1999).

[OV03] M. Ohlberger and J. Vovelle. Error estimate for the approximation of non-linear conservation laws on bounded domains by the finite volume method, Preprint 03-32, Mathematisches Institut, Freiburg (2003). 
[OMF+00] K.M. Olson, P. MacNeice, B. Fryxell, P. Ricker, F.X. Timmes, and M. Zingale. PARAMESH: A parallel, adaptive mesh refinement toolkit and performance of the ASCI/FLASH code, in Proceeding of the AAS 195th Meeting (2000).

[Ope02] OpenMP-Team. Official OpenMP Specifications, C/C ++ Version 2.0 (2002), available from WWW: www.openmp.org/drupal/ $\mathrm{mp}$-documents/cspec20.pdf.

[Ott96] F. Otto. Initial-boundary value problem for a scalar conservation law, [J] C. R. Acad. Sci., Paris, Sér. I 322, No.8, 729-734 (1996).

[Pet99] N.A. Peterson. Hole-cutting for three-dimensional overlapping grids, SIAM J. Sci. Comput., Vol. 21, No. 2, 646-665 (1999).

[PL92] T.J Poinsot and S.K. Lele. Boundary conditions for direct simulations of compressible viscous flows, J. Comput. Phys., 101: 104-129 (1992).

[PHPM88] J. Probert, O. Hassan, J. Peraire, and K. Morgan. Transient adaptive methods for moving boundary problems, Ed.: Gruber, Proceeding of the 5. International Symposium on Numerical Methods in Engineering (1988).

[RK97] B.D. Raghunathan and R.G. Kenny. CFD simulation and validation of the flow within a motored two-stroke engine, SAE Paper No. 970359, Society of Automotive Engeneers, Warrendale, Pa. (1997).

[RKPM01] H. Rosskamp, A. Klimmek, P. Pretzsch, and M. Mugele. Scavenge Loss mechanisms and their driving forces in loop-scavenged highperformance two-stroke engines, SAE Paper No. 2001-01-1826/4247, Society of Automotive Engeneers, Warrendale, Pa. (2001).

[RS95] M. Rumpf and B. Schupp. Visualization of parallel data based on procedural access, In Proc. MathVis Conference, Berlin (1995).

[SM95] P. Schiano and A. Matrone. Parallel CFD applications: experiences on scalable distributed multicomputers, in: Parallel Computational Fluid Dynamics, New Trends and Advances, N. Satofuka, J. Periaux, A. Ecer (eds.), North Holland (1995).

[SG97] H. Schlichting and K. Gersten. Grenzschicht-Theorie, Springer-Verlag, Berlin (1997).

[Sch01] P. Schott. Dreidimensionale Strömungssimulation in der Wettervorhersage, Diplomarbeit, Mathematisches Institut, Universität Freiburg (2001). 
[Sch99] B. Schupp. Entwicklung eines effizienten Verfahrens zur Simulation kompressibler Strömungen in 3D auf Parallelrechnern, Doctoral Dissertation, Mathematisches Institut, Freiburg (1999).

[Sch97] R. Schwörer. Entwicklung und Parallelisierung von Finite Volumen Verfahren zur Lösung der kompressiblen Navier-Stokes Gleichungen in 2D, Diplomarbeit, Mathematisches Institut, Freiburg (1997).

[Sed72] L.I. Sedov. A course in continuum mechanics, Volume II, Physical foundations and formulations of problems, Wolters-Noordhoff Publishing, Groningen (1972).

[ST67] I.M. Singer and J.A. Thorpe. Lecture Notes on Elementary Topology and Geometry, Springer-Verlag, New York (1967).

[SB87] J.L. Steger and J.A. Benek. On the use of composite grid schemes in computational aerodynamics, Computer Meth. Appl. Mech. Eng., 64, 301-320 (1987).

[TG99] A.S. Tanenbaum and J. Goodman. Computerarchitektur, Prentice Hall, München (1999).

[Tre00] D. Trescher. Theorie und Numerik Transparenter Randbedingungen, Diplomarbeit, Mathematisches Institut, Universität Freiburg (2000).

[Tru89] E. Truckenbrodt. Fluidmechanik, Band I, Grundlagen und elementare Strömungsvorgänge dichtebeständiger Fluide, Springer-Verlag, Berlin (Dritte Auflage, 1989).

[vdV94] E. van der Velde. Concurrent scientific computing, Springer-Verlag, New York (1994).

[Vdi97] Verein Deutscher Ingenieure. VDI-Wärmeatlas: Berechnungsblätter für den Wärmeübergang, Hrsg. VDI-Gesellschaft Verfahrenstechnik und Chemieingenieurwesen, Springer-Verlag, Berlin (Achte Auflage, 1997).

[VH93] R. Vilsmeier and D. Hänel. Adaptive methods on unstructured grids for Euler and Navier-Stokes equations, Computer Fluids, 22(4/5): 485-499 (1993).

[WL97] Y. Wada and M.-S. Liou. An accurate and robust flux splitting scheme for shock and contact discontinuities, SIAM J. Sci. Comput., 18, No. 3: 633-657 (1997).

[Wer00] A. Werner. 3D simulation of instationary turbulent flow and combustion in internal combustion engines, in E. Krause, W. Jäger (Eds), High performance computing in Science and Engineering, Springer (2000). 
[Wie95] M. Wierse. Higher Order Upwind Schemes on Unstructured Grids for the Compressible Euler Equations in Timedependent Geometries in 3D, Doctoral Dissertation, Mathematisches Institut, Freiburg, SFB256 Preprint 393, Bonn (1995).

[WK96] M. Wierse and D. Kröner. Higher order upwind schemes on unstructured grids for the nonstationary compressible Navier-Stokes equations in complex time-dependent geometries in 3D, Preprint Nr.2, Universität Freiburg (1996).

[WKM+98] M. Wierse, D. Kröner, A. Müller, B. Schupp, and R. Schwörer. Simulation of a 3-D piston driven flow, R. Friedrich (ed.) et al., Computation and visualization of three-dimensional vortical and turbulent flows. Proc. of the 5th CNRS-DFG workshop on Numerical flow simulation, München, Germany, Dec. 6-7, 1996. Wiesbaden: Vieweg. Notes Numer. Fluid Mech. 64, 333-349 (1998).

[WR92] M. Wierse and M. Rumpf. GRAPE, Eine interaktive Umgebung für Visualisierung und Numerik, In Informatik, Forschung und Entwicklung, Vol. 7, 145-151 (1992).

[ZSLC04] Y. Zeng, S. Strauss, P. Lucier, and T. Craft. Predicting and optimizing two-stroke engine performance using multidimensional CFD, SAE Paper No. 2004-32-0039, Society of Automotive Engeneers, Warrendale, Pa. (2004). 


\section{Index}

adiabatic exponent, 29, 39-41, 119, 126, 130, 133, 138, 142, 146, 150, 163, 165

air-fuel ratio, 15, 22, 39

bore, 12,171

boundary conditions, 33-35, 55, 67, 108, 116, 120, 123, 126, 129, 133, 134, 137, 142, 150, 161, 169,173

boundary fitting, 95, 152

CFD, 3, 4, 6, 16, 19, 20, 25, 101, 191

CFL-condition, 58, 72, 88, 114

combustion, 2, 10, 14, 15, 20, 21, 26, 163, 172, 191, 199, 204

stoichiometric combustion, 15, 22, 39

compression ratio, 12, 26, 163, 171

connecting rod, 13, 21, 85, 107, 170

convective flux, 32, 50, 52, 57, 131

AUSMDV, 50, 52, 60

curved interface method, 78, 79, 203

Cycle of Operation, 10

delivery ratio, 13, 99, 173, 178

distributed memory, 102, 105

enhanced initial data, 97, 168, 176

EOC, 113, 117, 120, 125, 131, 135, 140, 144

equation of state, $27,29,32,36,38$, $56,60,63,70,108,172$

Euler equations, 32, 45, 60, 61, 114, 149

finite volume scheme, 45, 47, 55, 79, $80,83,87,93,103,113$

fitted interface method, 78 grid merging, 47, 65, 76, 79, 84, 145, 167, 175

heat capacity, 28, 30, 36, 38, 39, 41, 63, $130,133,138,142,145,160$

initial conditions, 32-34, 36, 61, 150, 165,172

integral form, 45, 54

Jante test, 24, 182, 190, 191

load balancing, 105

local mesh adaption, 91, 105, 148, 194

local time-stepping, 87, 89, 104, 114, 176, 192

Navier-Stokes equations, 4, 6, 30, 32, 34, 39, 45, 47, 54, 60, 83, 129, $141,145,159$

OpenMP standard, 103, 173, 203

Otto Cycle, 20, 40, 163

partitioning, 103-105, 173, 176

piston position, 13, 74

port plan layout, 191

Prandtl number, 4, 30, 137, 138

purity, 14, 23-25, 186

purity at exhaust port entry, 23, 24, 186

relaxation scheme, 5, 60

Reynolds number, 4, 30, 36, 129

scavenge ratio, $13,18,19,23,35,100$, 147, 176, 178, 186

scavenging, 2, 6, 15, 22, 23, 25, 85, 99, 107, 166, 167, 179, 181, 186, 190, 197, 200

loop scavenging, 15, 16, 25, 186 
scavenging efficiency, 23, 24, 99, 186

shared memory, 103, 105, 173

short-circuiting, 10, 15, 18, 19, 23, 24, 167, 181, 186, 199, 200

snapper algorithm, 73, 74, 76, 89, 95, 105, 168

squish velocity, 26

stroke, 12,171

thermal conductivity, 30-32, 39, 40, 42, 129, 130, 133, 138, 142, 145,160

trapping efficiency, 14, 18, 19, 23, 35, 99, 100, 147, 173, 176, 178, 186, 195, 199

virtual splitting method, 70, 145

viscosity, 30, 32, 39, 40, 42, 129, 130, $133,138,142,145,160$

viscous flux, 52, 53, 58 